\title{
Search for the $\Theta_{5}(1540)^{+}$
}

\section{Strange-Pentaquark Candidate in $e^{+} e^{-}$}

\section{Annihilation, Hadroproduction and Electroproduction with the BABAR \\ Detector}

\author{
Jonathon P. Coleman
}

May 2005

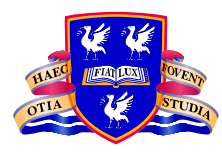

THE UNIVERSITY

of LIVERPOOL

Department of Physics

Thesis submitted to University of Liverpool for the degree of Doctor of Philosophy 


\section{Abstract}

Since early in 2003, several experiments have presented evidence for the existence of a positive strangeness baryon state of mass around $1540 \mathrm{MeV} / \mathrm{c}^{2}$ and width $<8 \mathrm{MeV}$, the $\Theta(1540)$, which decays to $K^{+} n$ and $K^{0} p$. Such a state has minimum quark content $u d u d \bar{s}$ and consequently has been interpreted as the $S=+1$ member of the anti-decuplet of pentaquark states proposed by Diakonov et al. Subsequently, the NA49 experiment presented evidence for the $S=-2$ member of the anti-decuplet, the $\Xi_{5}(1860)^{--}$, but this has yet to be observed in any other experiment.

Results from the search for the production of the $\Theta(1540)$ memember of the antidecuplet of pentaquark states using data from $e^{+} e^{-}$collisions obtained with the BABAR detector at the PEP-II Collider are presented. No signal is observed, and cross section limits for the $\Theta(1540)$ are given; these prove to be well below the cross section values for ordinary baryons of similar mass.

In addition, a search has been carried out for the electroproduction of the $\Theta(1540)$ in the material of the BABAR detector. Event selection procedures are discussed in detail, the results of this search are presented, and are discussed in the light of several other experiments. 


\section{The Author's Contribution}

The analysis of the data contained in this thesis has been carried out independently by the author. In addition to the analysis presented here, as a member of the BABAR Collaboration I have also been involved with DAQ operations and the online group during the Run period beginning Fall 2002 and ending Spring 2003. This involved being the primary oncall contact person for the online group on a $24 / 7$ basis. In this capacity I was involved in the daily routine frustrations and upkeep of the online system, which typically functions at an efficiency of $>98 \%$.

In addition to the above I have investigated the anomalies that can be seen in $K_{S}^{0}$ reconstruction, selection, and vertexing. Generally in $B A B A R$ there is a very conservative selection of $V^{0} \mathrm{~s}$ which involves an invariant mass cut on the vertexed pion pairs, allowing the kinematics of reconstructed B candidates to separate signal from background for B decays of interest. However, to understand the general behaviour of the $K_{S}^{0}$ in an inclusive environment this is less than ideal. I developed a new inclusive selection procedure which has since become one of the standard selections.

The improved purity and high efficiency that my selection achieved allowed the study of systematic behaviour of track reconstruction. From detailed study of the invariant mass of pion pairs, it became apparent that the material description used in reconstruction was inadequate. I carried out a study in which the track reconstruction was repeated using different models for the material composition of the silicon detectors (SVT), support tube, and drift chamber $(\mathrm{DCH})$ walls. The changes resulted in different energy loss corrections to the charged tracks, and hence affected the reconstructed $K_{S}^{0}$ mass value as a function

of vertex position. The modified material description led to the $K_{S}^{0}$ mass values agreeing with the PDG value at the $30 \mathrm{keV}$ level independently of vertex radius, which gave an order-of-magnitude improvement on previous performance. This has motivated further study of mass effects for other particle types (e.g. $\Lambda, D^{0}, \Lambda_{c}$ ) and should lead to a much better understanding of the BABAR mass scale.

\section{Acknowledgments}

The successful completion of the work contained within this thesis was only made possible through the efforts of many people, the majority of whom are the BABAR collaboration, I would like to thank all of them.

Bill Dunwoodie deserves a special mention, for his sharing of ideas, support and friendship. I wish to thank my supervisor Christos Touramanis, who was always there to provide support in whatever way was appropriate and to the BABAR group at Liverpool University for their constant support. 
I would like to thank David Leith for all that he has done. I also want to thank both David and Blair Ratcliff for their encouragement and financial support.

I am grateful to the members of Group B, all of whom have shared their time, ideas and have made my time at SLAC a source of inspiration.

Finally there are many people (far too many to mention) who have extended the hand of friendship and support to me during my time on BABAR. I would like to thank my parents and family for putting up with me, and for the encouragement and support that they have given me over many years. 


\section{Contents}

1 Introduction and Motivation $\quad 1$

1.1 QCD, Quarks and Exotic Baryons . . . . . . . . . . . . . . 1

1.2 The Pentaquark Revival; Prediction of the $\Theta_{5}^{+} \ldots \ldots \ldots \ldots$

1.3 First Experimental Evidence for the $\Theta_{5}(1540)^{+} \ldots \ldots \ldots$. . . . . . 7

1.4 Other Claimed Observations . . . . . . . . . . . . . . . . . . . . . 9

1.4 .1 The $\Theta_{5}(1540)^{+} \ldots \ldots \ldots \ldots$. . . . . . . . . . . . 9

1.4 .2 The $\Xi_{5}(1862) \ldots \ldots \ldots \ldots \ldots \ldots$

1.4 .3 The $\Theta_{c}(3100) \ldots \ldots \ldots \ldots \ldots 11$

1.5 Negative Searches . . . . . . . . . . . . . . . . . . 11

1.6 Present Status and Future Outlook . . . . . . . . . . . . . . . . . . 14

1.7 Motivation and Thesis Outline . . . . . . . . . . . . . 16

2 The BABAR detector $\quad 18$

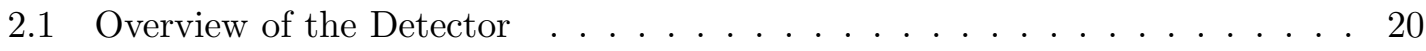

2.2 Silicon Vertex Tracker $(\mathrm{SVT}) \ldots \ldots \ldots \ldots \ldots$. . . . . . . . . . 21

2.3 Drift Chamber $(\mathrm{DCH}) \ldots \ldots \ldots \ldots \ldots$

2.4 Detector of Internally Reflected Cherenkov Radiation (DIRC) . . . . . . . . 27

2.5 ElectroMagnetic Calorimeter (EMC) . . . . . . . . . . . . . 30

2.6 Instrumented Flux Return (IFR) . . . . . . . . . . . . . . . . 32

2.7 Trigger . . . . . . . . . . . . . . . . . . . 34

3 Search for $\Theta_{5}(1540)^{+}$Production in $e^{+} e^{-}$Interactions 36

3.1 Introduction . . . . . . . . . . . . . . . . . . 36

3.2 Data Set . . . . . . . . . . . . . . . . . 36

3.3 Event Preselection . . . . . . . . . . . . . . . . . . . . . 38

3.3.1 Reconstruction and Selection of Inclusive $K_{S}^{0}$ Candidates . . . . . . 38

3.3.2 Individual Track Candidate Selection . . . . . . . . . . . . . . . . . . 41

3.4 Proton Identification . . . . . . . . . . . . . . . . . . . . . 42

3.5 Search for $\Theta_{5}^{+}(\mathbf{1 5 4 0}) \rightarrow \boldsymbol{p} \boldsymbol{K}_{S}^{\mathbf{0}} \ldots \ldots \ldots \ldots \ldots$. . . . . . . . . 44

3.5.1 Event Selection and Analysis . . . . . . . . . . . . . 44

3.6 Monte Carlo Samples . . . . . . . . . . . . . . . . . . . . . 51

3.7 Cross Sections and Upper Limits . . . . . . . . . . . . . . . . . . . . 51 
3.7.1 The $\Lambda_{c}^{+}$Differential Cross Section . . . . . . . . . . . . 54

3.7.2 Estimating 95\% C.L. Limits on the $\Theta^{+}$Cross Section . . . . . . . 57

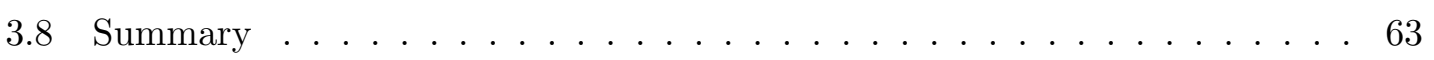

4 Search for $\Theta(1540)^{+} \rightarrow K_{S}^{0} p$ in Electro- and Hadro-production $\quad 67$

4.1 Motivation . . . . . . . . . . . . . . . 67

4.2 Overview of the Analysis and Summary of Results . . . . . . . . . . . . . 69

4.3 The Beampipe and Inner Detector Region . . . . . . . . . . . . . . . 72

4.4 Initial Selection of Candidate Events . . . . . . . . . . . . . . . . . . 74

4.4 .1 Proton Identification . . . . . . . . . . . . . . . . . . 76

4.4.2 Rejection of Misidentified Protons . . . . . . . . . . . . . 78

4.4 .3 Pion Identification . . . . . . . . . . . . . . . . 80

4.4.4 Tightening the $\left(K_{S}^{0}, p\right)$ Candidate Selection Criteria . . . . . . . . 82

4.5 Detector Tomography Using $K_{S}^{0} p$ Vertices . . . . . . . . . . . . . . . . 85

4.6 Search for $\Theta_{5}(1540)^{+} \rightarrow K_{S}^{0} p$ Using all Candidates . . . . . . . . . . . . . . 93

4.6.1 The Inclusive $K_{S}^{0} p$ Invariant Mass Distribution . . . . . . . . . . . . 93

4.6.2 The Inclusion of Additional Charged Tracks . . . . . . . . . . . . . . 94

4.6.3 The Identification of Associated Charged Tracks . . . . . . . . . . . 94

$4.7\left(K_{S}^{0}, p\right)$ Electroproduction in Be and $\mathrm{Ta} \ldots \ldots . \ldots . \ldots . . \ldots 2$

4.7.1 The Inclusive $K_{S}^{0} p$ Invariant Mass Distribution . . . . . . . . . . . . 107

4.7.2 The Inclusion of Additional Charged Tracks . . . . . . . . . . . . . . 107

4.7.3 The Identification of Associated Charged Tracks . . . . . . . . . . 107

4.8 Comparison to Other Experiments . . . . . . . . . . . . . . . . 114

4.8.1 HERMES [49] . . . . . . . . . . . . . . . . . . . . . 114

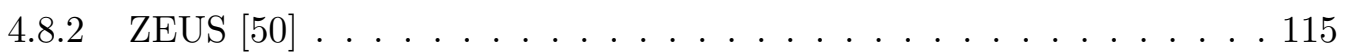

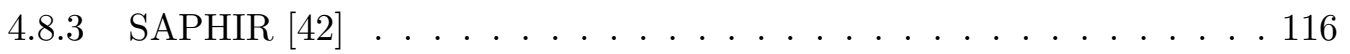

$\begin{array}{lll}5 & \text { Conclusion } & 118\end{array}$ 


\section{Chapter 1}

\section{Introduction and Motivation}

\subsection{QCD, Quarks and Exotic Baryons}

It seems to be well-established that Quantum Chromodynamics (QCD) is the correct theory of the strong interactions. For example, it has been tested extensively in many experiments at high momentum transfer, where asymptotic freedom justifies the use of perturbation theory, and no shortcoming has been revealed. Hadrons are then interpreted as bound states of quarks held together by gluon-mediated, non-Abelian gauge interactions. However an immediate corollary is that there is no a priori reason for mesons to exist only in $q-\bar{q}$ configurations and for baryons to occur with only triquark structure; mesons made up of two quarks and two anti-quarks, or multiple gluons (a "glueball"), or a gluon and a $q-\bar{q}$ pair (a quark-gluon hybrid) might also exist, and similarly the possibility of baryon states composed of four quarks and an anti-quark cannot be ruled out. States with this latter configuration are referred to as pentaquark states, and this thesis is concerned with the search for evidence of the production of one such state, the $\Theta_{5}(1540)$, in the very large data sample acquired to date using the BABAR detector at SLAC. This state is a positive strangeness $(S=+1)$ baryon, and as such it must have minimal quark content $[u u d d \bar{s}]$. It is thus beyond the "standard" three-quark model of baryons, and in this sense is considered to be manifestly "exotic".

However, in this regard it should be noted that in the seminal papers of Gell-Mann [1] and Zweig [2], in addition to the $q \bar{q}$ structure of mesons and the $q q q$ structure of baryons, there was also discussion of meson structures consisting of two quarks and two anti-quarks, and of baryons made up of four quarks and an anti-quark (pentaquark states). [Of course, Zweig didn't call them "quarks"; he used the term "aces", which seems strange since normally you would consider there to be four aces; either he wasn't playing with a full deck, or he had charm up his sleeve!].

In the quark model [1], baryons and mesons were considered to be built out of unitary triplets considered to be fundamental objects which were NOT to be identified with known particles. The triplet consisted of a quark doublet $(u$ and $d)$ and singlet $(s)$ with charge $\left(\frac{2}{3} e, \frac{-1}{3} e, \frac{-1}{3} e\right)$, strangeness $(0,0,-1)$ and baryon number $\left(\frac{1}{3}, \frac{1}{3}, \frac{1}{3}\right)$ respectively, in a scheme 
which Gell-Mann chose because of its simplicity and elegance [1]. This was the outcome of a search for some fundamental explanation as to why the broken $\mathrm{SU}(3)$ symmetry scheme ("The Eightfold Way") [3, 4, 5] was so successful in describing the spectroscopy and quantum numbers of the then-known meson and baryon states.

Broken SU(3) grew out of the attempts to generalize the successful use of isospin in the description of the charge independence of strong interaction processes to include hypercharge, $Y=S+B$, where $B$ is baryon number. In the treatment of isospin, the operators corresponding to the three components of the isospin vector were considered to be the generators of the algebra of SU(2). Similarly, with the addition of the hypercharge operator $Y$, and four other operators (corresponding to $u$-spin and $v$-spin), the resulting eight operators were considered to be the generators of the algebra of SU(3) (hence Eightfold Way). Just as isospin multiplets corresponded to the irreducible representations of $\mathrm{SU}(2)$, there were now supermultiplets corresponding to the irreducible representations of $\mathrm{SU}(3)$, and just as the mass degeneracy of the $\mathrm{SU}(2)$ multiplets was broken as a result of the electromagnetic interaction (i.e. charge), so the mass degeneracy of the $\mathrm{SU}(3)$ multiplets was broken as a result of the strong interaction (i.e. hypercharge). In the unitary triplet realization, such symmetry breaking would result from a mass difference between the $s$ and $(u, d)$ quarks [1].

The quark model now inherited all of the successes of the broken SU(3) symmetry scheme, and these had been indeed remarkable.

The lowest SU(3) multiplets are a singlet and an octet. For the baryon states, the singlet was discarded [3] (with the inclusion of colour, the ground state singlet is forbidden by Fermi statistics), but the known states $[N, \Lambda, \Sigma, \Xi]$ were consistent with a $J^{P}=1 / 2^{+}$ octet. The pseudoscalar mesons $[K, \pi, \bar{K}]$ also were consistent with the predicted octet structure, but the isoscalar state was missing. This expected state (the $\eta$ ) was found in the predicted mass region shortly thereafter, as was the singlet state (the $\eta^{\prime}$ ) some time later. An octet of vector meson states was predicted also, although only the $\rho$ and the $\omega$ were known at the time. The expected strange mesons, the $K^{*}(892)$ and $\bar{K}^{*}(892)$ were first reported at almost the same time as the writing of [3], thus completing the octet, and the predicted vector meson singlet state, the $\phi(1020)$, was found some time later. These successes were indeed impressive. However, by far the most remarkable outcome concerned the $\Omega^{-}$baryon. The isospin $3 / 2 \Delta(1232)$ resonance decays to $N \pi$ final states. If the baryon and pseudoscalar meson octets are to combine to yield a multiplet containing the $\Delta$, only the 10 (decuplet) and 27 representations survive. At the 1962 CERN conference, Gell-Mann made a prediction which proved to be truly astonishing in its accuracy [6]. After a rapporteur talk by Good he pointed out that if the $\Delta$ were to be included in the broken symmetry scheme, it would have to be part of a decuplet with approximately equal mass splitting between the states involved. Interestingly, he ruled out the 27 representation by stating: "The only supermultiplet that does not lead to non-existent resonances in the $K-N$ channels is the 10 representation..." (underline 
added, since he said in effect that the non-existence of resonant pentaquark states was the basis for his choice!). He conjectured that the $Y^{*}(1385)$, which was suspected to have $J^{P}=3 / 2^{+}$(later confirmed), might belong to this decuplet, so that the mass splitting would be $\sim 147 \mathrm{MeV} / \mathrm{c}^{2}$, leading to mass estimates of $\sim 1530$ and $\sim 1680 \mathrm{MeV} / \mathrm{c}^{2}$ for the missing $S=-2$ and $S=-3$ decuplet states. He suggested therefore that the $\Xi(1530)$ reported at the conference $\left(J^{P}=3 / 2^{+}\right.$established later) could belong to this decuplet, and that a search be carried out for the remaining decuplet state, the $\Omega^{-}$as he named it, with $S=-3, I=0$ and mass $\sim 1685 \mathrm{MeV} / \mathrm{c}^{2}$. He pointed out that it should decay weakly to $\Lambda K^{-}, \Xi^{0} \pi^{-}$(the mode in which it was later discovered [7]) and $\Xi^{-} \pi^{0}$, all of which have since been observed. He further suggested that it might explain "the old (emulsion) Eisenberg event" [8] (it did; see the section on the $\Omega^{-}$in [9]) and that it might be found in the reaction $K^{-} p \rightarrow K^{0} K^{+} \Omega^{-}$(it was; see [7]). The proceedings show no further discussion of this very public prediction, which must surely rank as one of the most prescient in the annals of high-energy physics.

Despite the discovery and classification of a very large number of meson and baryon states over the years subsequent to 1964, the "standard" quark model has proved to be remarkably successful in its ability to accommodate the bulk of the relevant mass and quantum number information.

In the meson sector, the treatment of mesons as $q \bar{q}$ systems bound in a relativized phenomenological QCD confining potential which incorporates chromodynamics [10] provides a reasonable description of the whole spectrum of known meson states, including those involving heavy quarks. In this model, the troublesome $a^{0}(980)$ and $f^{0}(980)$ scalar mesons are described as $K \bar{K}$ bound states with no isoscalar or strange meson partners [11]. An alternative bag-model description [12] finds that they can occur in a low-mass nonet of "cryptoexotic" $q q \bar{q} \bar{q}$ states, thus going beyond a $q \bar{q}$ description. However, this would imply the existence of strange and isoscalar members of such a nonet, the $\kappa$ and $\sigma$ respectively, and there is extensive debate as to whether these states actually exist.

One area of difficulty for the $q \bar{q}$ description involves the higher mass isoscalar mesons with $J^{P C}=0^{++}$; it appears that there are just too many such states. Lattice Gauge calculations (e.g. [13]) predict the existence of glueball states in this mass region, the lowest mass state having $J^{P C}=0^{++}$with mass expected to lie between 1.4 and $1.8 \mathrm{GeV} / c^{2}$. As such it should mix with the regular $0^{++}$states, perturbing the mass spectrum and leading to too many observed states with these quantum numbers. In fact a good candidate for such a state consisting mostly of "glue" has been observed in $J / \psi$ radiative decay to $K \bar{K}$ and $\pi^{+} \pi^{-}$[14]. The presence of such states indicates the need for a description of mesons which goes beyond the standard $q \bar{q}$ model.

The states described as quark-gluon hybrids can have exotic quantum numbers i.e. quantum numbers which cannot be generated in a $q \bar{q}$ model, such as $J^{P C}=1^{-+}$. Gluon flux-tube models [15] predict that there should exist such a state with mass $\sim 1.9 \mathrm{GeV}$, and Lattice Gauge calculations yield similar predictions [16]. Bag models [17] predict lower 
mass states, of which one has $J^{P C}=1^{-+}$. In recent, years partial wave analyses of data from BNL $[18,19]$ have yielded evidence for the observation of such exotic states, and this provides the most convincing demonstration to-date for the existence of meson states not amenable to a $q-\bar{q}$ description.

In the light-quark baryon sector, spin and flavour are combined to yield a flavour-spin $\mathrm{SU}(6)$ representation of the spectroscopy. The baryon states are composed of three quarks, each of which is assumed to be a colour triplet, with all baryons assumed to be colour singlets. The allowed state vectors are thus anti-symmetric in colour, and symmetric in space-spin-flavour, and in this way the quark configurations satisfy Fermi statistics. All of the known (i.e. with at least 3 stars in PDG evaluation) baryon states up to $\sim 2 \mathrm{GeV}$ are accommodated in this scheme, and the non-relativistic three-quark potential model of Isgur and Karl can also explain the occurrence of "missing states" on the basis of their highly inelastic decay characteristics [20]. In fact, in the review article by Hey and Kelly [21] it is pointed out that the successful description of the known baryon states in terms of confined triplets of spin-1/2 quarks with a hidden colour degree of freedom is perhaps the most significant outcome of all the attempts at describing the spectroscopy (and couplings) of the baryonic excitations. However, in the context of QCD, the apparent absence of baryons with composition $q q q q \bar{q}$ or $q q q g$ is not understood.

The most obvious way to detect the need for the former would be to identify resonant structure in the $K N$ system, since an $S=+1$ baryon must at minimum contain five quarks, as discussed previously. Since the early days of the quark model, such evidence has been sought in the Partial Wave Analysis (PWA) of $K N$ elastic, charge exchange and inelastic scattering data. The results of the searches for $Z^{*}$ states, as they were called, are summarised briefly in ref. [21]. Only the $P_{01}$ and $P_{13}$ amplitudes show some evidence of structure (in the mass region 1.8-1.9 GeV/c $c^{2}$ ), but it is concluded that there has been no convincing demonstration of resonant behaviour. By 1986 the Particle Data Group had had enough, and in its 1986 review [22] officially put the subject of $Z^{*}$ 's to sleep with the following comment:

"..the (PWA) results permit no definite conclusion - the same story heard for 15 years. The standards of proof must simply be much more severe here than in a channel in which many resonances are already known to exist. The general prejudice against baryons not made of three quarks and the lack of any experimental activity in this area make it likely that it will be another 15 years before the issue is decided."

The $Z^{*}$ listings appeared for the last time in this issue, and starting with PDG1988 [23], only a reference to the 1986 edition was included.

After that, the subject of exotic baryons did not receive much attention except from a few theorists motivated by the old chiral soliton ideas due to Skyrme [24]. 


\subsection{The Pentaquark Revival; Prediction of the $\Theta_{5}^{+}$}

In 1997 Diakonov et al. published a paper [25] making a remarkable prediction concerning the existence of a positive strangeness baryon state just above $K N$ threshold in mass $\left(\sim 1.53 \mathrm{GeV} / c^{2}\right)$ and of extremely narrow width $(\Gamma<15 \mathrm{MeV})$. As discussed previously, such a state is manifestly exotic and must have a minimal content of four quarks and an anti-quark $(\bar{s})$. The predictions were based on the generalization of a chiral soliton model [24], in which nucleons are viewed as solitons of the pion field. Quantization of the rotations of this field in ordinary and flavour $\mathrm{SU}(3)$ space leads to a baryon ground state which is an octet with spin $1 / 2$, and to a first excited state which is a spin $3 / 2$ decuplet, just as seems to be the case in nature. In the case of three flavours, the next excitation corresponds to an anti-decuplet with spin $1 / 2$. The structure of this decuplet is shown in fig.1.1, with the exotic $S=+1$ state occupying the apex of the triangle. The states at the extreme edges of the base of the triangle are also manifestly exotic with minimal quark content as shown. As indicated, the mass splitting between the isospin multiplets of different strangeness is linear; in reference [25], it is estimated to be $\sim 180 \mathrm{MeV} / \mathrm{c}^{2}$. The overall mass scale was defined by identifying the nucleon member of the decuplet with the $N(1710)$ resonance [9], and this leads to an estimation of the $\Theta_{5}$ mass of $\sim 1.53 \mathrm{GeV} / c^{2}$. A subsequent calculation of the width of this state leads to the estimate that it should be $\sim 15 \mathrm{MeV}$, which, if correct, should make it amenable to experimental detection provided the cross section for production is large enough.

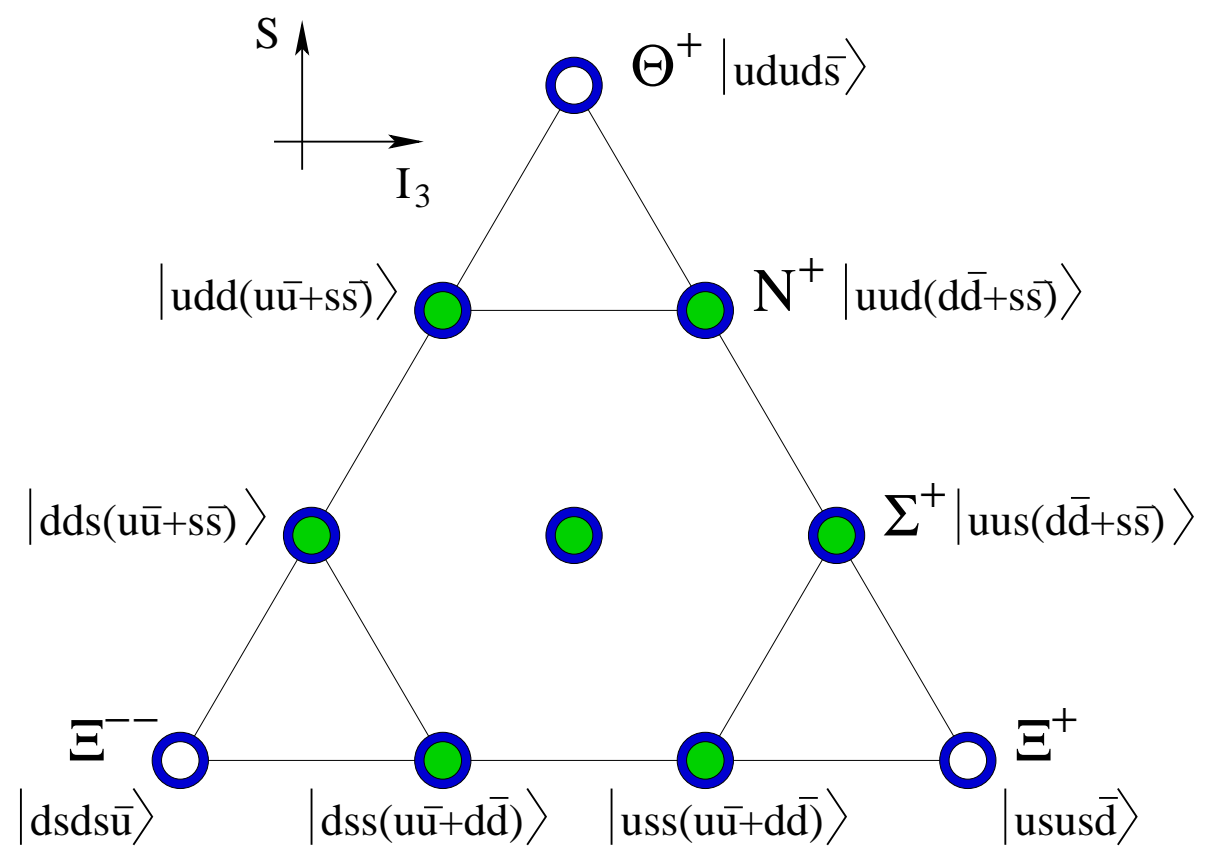

Figure 1.1: Quark structure of the anti-decuplet (annuli) and octet(filled circles) that are generally assumed for the lowest mass pentaquarks. The vertical axis is the strangeness and the horizontal axis is the isospin. The quark content of the decuplet members is shown. 
On p.312 of ref. [25] there is a comment to the effect that data from the LASS experiment might be used to look for the $\Theta_{5}^{+}$. This was in fact done [26] in 1997 using data on the reaction $K^{+} p \rightarrow \pi^{+} K^{+} n$ at incident momentum $11 \mathrm{GeV} / c$, but no signal was observed. Old, but high quality, bubble chamber data on e.g. the reaction $K^{+} p \rightarrow \pi^{+} K^{0} p$ in the 1 $\mathrm{GeV} / c$ momentum region [27] also reveal no signal, and suggest cross section values less than $\sim 10 \mu \mathrm{b}$. Representative Dalitz plots with the $\Theta_{5}$ region indicated can be found in the PDG2004 article by George Trilling [28]; it is quite clear that there is no evidence of $\Theta_{5}$ production.

For more than five years after the publication of refce. [25] there was no experimental evidence to support the prediction of the $\Theta_{5}$, but the situation began to change rather dramatically in the Fall of 2002 when the LEPS Collaboration claimed to observe photoproduction of a $\Theta_{5}$ candidate [29] (see section 1.3 below).

Then, during a Pentaquark Workshop at Jefferson Lab. in November 2003, K.Kadija, representing the NA49 Collaboration, presented evidence for the production of a $\Xi_{5}^{--}$ pentaquark candidate and a neutral partner in $p-p$ interactions at a c.m. energy of 17.2 $\mathrm{GeV}$ (later published in refce. [30]). If this state is interpreted as belonging to the antidecuplet predicted by Diakonov, the mass $\left(\sim 1.862 \mathrm{GeV} / c^{2}\right)$ and width $(<18 \mathrm{MeV})$ values are much smaller than those predicted in refce. [25] ( $2.07 \mathrm{GeV} / c^{2}$ and $>140 \mathrm{MeV}$ resp.). In a subsequent paper, Diakonov no longer used the $\mathrm{N}(1710)$ to set the absolute mass scale for his predictions, but used the mass values of the $\Theta_{5}$ and $\Xi_{5}$ to define a new decuplet central mass and mass splitting $\left(\sim 108 \mathrm{MeV} / \mathrm{c}^{2}\right)$ [31]. The reduction of the splitting from the original expected value of $180 \mathrm{MeV} / \mathrm{c}^{2}$ [25] can be reproduced by increasing the value of the nucleon sigma term used in the calculation, and arguments are given which indicate that the NA49 width limit is reasonable if the true width of the $\Theta_{5}$ is $<3 \mathrm{MeV}$, and the two states are members of the same anti-decuplet.

Stimulated no doubt by the flurry of experimental activity on the pentaquark front, other models of the "quark cluster" type soon appeared. The first of these, due to Karliner and Lipkin [32], divided the pentaquark constituents into a di-quark and a tri-quark cluster with the quarks of identical flavour in different clusters. Each cluster has isospin zero and is a colour non-singlet (seperating the pairs of identical flavor); one unit of orbital angular momentum then yields $I J^{P}=01 / 2^{+}$as expected for the lowest anti-decuplet, and the centrifugal barrier keeps the clusters beyond the range of the repulsive color-magnetic force. The individual clusters stay together as a result of colour-electric forces. The model yields a $\Theta_{5}$ mass estimate of $\sim 1.59 \mathrm{GeV} / c^{2}$, and an anti-decuplet mass splitting which is only $\sim 50 \mathrm{MeV} / c^{2}$. Nevertheless, it is a quark-based model which leads to a resonant $\mathrm{S}=+1$ baryon state in the vicinity of $K N$ threshold.

A second model of this type is due to Jaffe and Wilczek [33]. The $\Theta_{5}$ is described in terms of two $u-d$ diquarks and a bachelor $\bar{s}$ quark. The ground state diquark-diquarkanti-quark configuration leads to a degenerate octet and anti-decuplet whose symmetry is broken as a result of the strange quark mass, leading to mixing of the two multiplets. 
Incorporating the $\Theta_{5}$ as the $Y=2$ member of the anti-decuplet, this leads to a somewhat different spectroscopy than that of refce. [25], and in particular yields a $J^{P}=1 / 2^{+}$nucleon state at a mass lower than the $\Theta_{5}$ which is associated with the broad Roper resonance. However, the predicted mass of the $\Xi_{5}$ state is more than $100 \mathrm{MeV}$ below the mass of the state claimed by the NA49 experiment.

Finally, it should be possible to employ Lattice Gauge techniques to investigate the possible existence of an exotic pentaquark resonant state in the vicinity of $K N$ threshold. In a recent paper [34], the present status of such calculations is discussed and it is concluded that there is as yet no evidence favouring the existence of any such state. However the paper cautions that "absence of evidence" should not be considered to be "evidence of absence" at the present early stage of these efforts.

Since the claim of evidence for $\Theta_{5}$ production, many models generating estimates of production cross section rates in photoproduction and hadroproduction reactions have been presented in the literature. Cross section estimates range from a fraction of a nb to several hundred nb in photoproduction, and from a fraction of a microbarn to several millibarns in hadroproduction, depending on the reaction and details of the model. A sampling of such calculations can be found in refces. [35] and [36], and in certain of the references listed in these papers. Some of the calculations yield results which are clearly nonsense e.g. for the reaction $K^{+} p \rightarrow \pi^{+} \Theta_{5}^{+}$predicted cross sections are $\sim 1.5 \mathrm{mb}$ at low beam momentum, whereas the published data [27] clearly indicate that such values are totally untenable; someone just didn't do their homework. Since the experiments claiming observation of the $\Theta_{5}$ have for the most part provided no reliable cross section estimates it seems rather pointless to discuss such model calculations in any detail. The few experimental cross section measurements will be discussed in section 1.4 below.

\subsection{First Experimental Evidence for the $\Theta_{5}(1540)^{+}$}

The first experimental evidence for the production of the $\Theta_{5}(1540)^{+}$was presented by Nakano at the PANIC 2002 Conference in Osaka in September, 2002 [29]. Data from LEPS at the SPring- 8 facility resulting from the interactions of back-scattered laser photons in a $\mathrm{CH}_{2}$ plastic scintillator start counter were used to select events corresponding to the reaction $\gamma n \rightarrow K^{-} K^{+} n$, with incident photon energy in the range $1.6-2.35 \mathrm{GeV}$. The final state neutron was identified on the basis of missing mass, and the missing mass recoiling against the $K^{-}$, after correction for Fermi motion of the target neutron, then represents the invariant mass of the $K^{+} n$ system. This distribution shows an excess of events in the vicinity of $1.54 \mathrm{GeV} / c^{2}$ above a background distribution obtained from $\gamma p \rightarrow K^{-} K^{+} p$ interactions in the experiment's $L H_{2}$ target. No numbers were given and no fits to the data distribution were shown; this section of the presentation was simply titled "Observation of a $S=+1$ Baryon Resonance". Inspection of the mass distribution shows 32 events in the mass region $1.500-1.5625 \mathrm{GeV} / c^{2}$ with a background of $\sim 11$ events, which would imply 

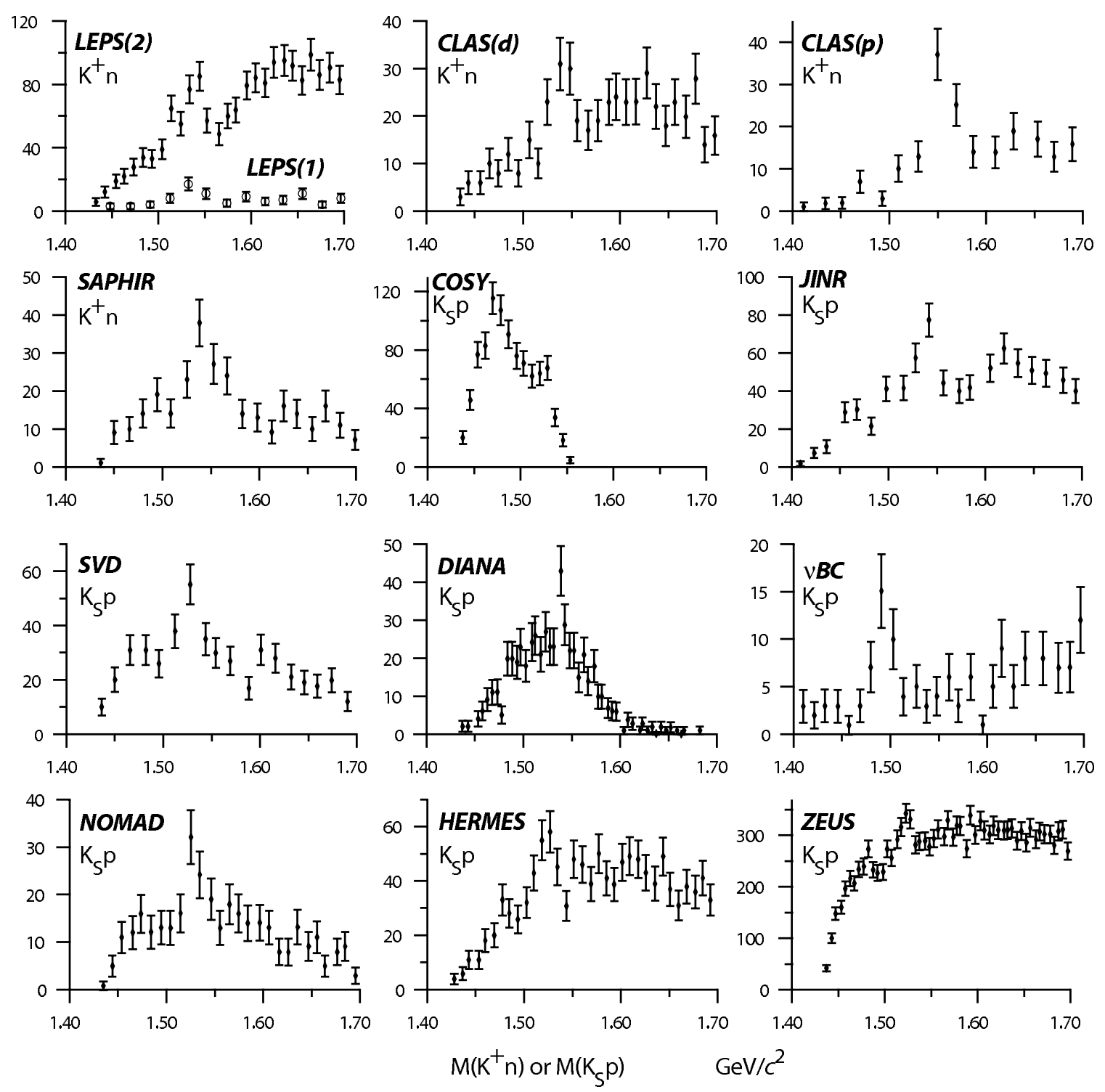

Figure 1.2: A compilation of the $K^{+} n$ or $K_{S}^{0} p$ mass distributions for the experiments listed in Table 1.1. Only the mass region from 1.4 to $1.7 \mathrm{GeV} / c^{2}$ is shown. Error bars represent statistical uncertainty only.

$\sim 6 \sigma$ significance. The bin size for the distribution shown was $12.5 \mathrm{MeV} / c^{2}$.

The final results of this analysis were published in July, 2003 [37]. The new plot had $20 \mathrm{MeV} / c^{2}$ bins, and contained 36 events in the region $1.510-1.570 \mathrm{GeV} / c^{2}$ with an estimated background of $17 \pm 2.8$ events. No fit was shown, the mass was estimated to be $1.54 \pm 0.01 \mathrm{GeV} / c^{2}$, the signal width was stated to be less than $25 \mathrm{MeV}$, and the significance claimed was $4.6_{-1.0}^{+1.2} \sigma$. The title of the paper is "Evidence for....", and whereas the PANIC presentation referred only to the pentaquark prediction of Diakonov et al., the PRL article acknowledges that it is also possible that the effect might be due to a molecular meson-baryon resonance. The mass distribution is shown in fig. 1.2 labelled as $\operatorname{LEPS}(1)$. 
Motivated by this result, the LEPS Collaboration repeated the experiment with a $L D_{2}$ target, and obtained much higher statistics for the same interaction [ignoring effects due to the different nuclear target material]. The results were presented by Nakano at QNP2004 in May, 2004 [38], but have not been published as yet. The distribution obtained is shown in fig. 1.2 labelled as $\operatorname{LEPS}(2)$; here it should be noted that the $\operatorname{LEPS}(2)$ distribution has $10 \mathrm{MeV} / c^{2}$ bins, whereas that for LEPS(1) has $20 \mathrm{MeV} / c^{2}$ bins, although the $y$-axis scale is in "Events". No numbers were presented; it was stated that the previous peak had been confirmed, that it was very unlikely that it was due to statistical fluctuation, and it was noted that the PDG had accorded it three stars. The total number of events in the mass region $1.51-1.56 \mathrm{GeV} / c^{2}$ is $\sim 338$, and the background in the region seems to be $\sim 230$ events; this would correspond to an excess of $\sim 7 \sigma$, although the shape of the peak looks rather ugly. Comparing the level of background to that of LEPS(1), it seems as though the total number of LEPS(2) events expected in this region should have been $\sim 480$, which would have given an excess of $\sim 16 \sigma$, i.e. although it is difficult to argue against the presence of a signal, the magnitude does not seem to track well with the increase in integrated luminosity, and this has to cause concern about the interpretation.

\subsection{Other Claimed Observations}

Dzierba et al. [39] have prepared a rather comprehensive survey of the experiments which have made contributions to the ongoing debate concerning the existence or non-existence of pentaquark states. The information presented in the following sub-sections is drawn for the most part from this article.

\subsubsection{The $\Theta_{5}(1540)^{+}$}

Fig. 1.2 is from ref. [39], and summarizes graphically the various claims for the observation of the $\Theta_{5}(1540)^{+}$pentaquark state, including those from the LEPS Collaboration discussed in section 1.3 above. The corresponding collaborations and reactions studied are summarized in Table 1.1, which also lists mass and width estimates, and claimed significance.

The first three rows of Table 1.1 result from photoproduction of the same exclusive final state on a virtual neutron target, with the signal appearing in the $K^{+} n$ invariant mass distribution $[29,37,38,40]$. The next two rows are from photoproduction of different exclusive final states on a proton target, with the signal again appearing in the $K^{+} n$ invariant mass distribution $[41,42]$. The quoted mass and width values appear to be consistent, and yield an average mass of $1544.2 \pm 3.6 \mathrm{MeV} / c^{2}$ for the $K^{+} n$ mode, with width $<20-25 \mathrm{MeV}$.

The remaining measurements are for the $K_{S}^{0} p$ system, but only for the exclusive final states of refs. [43] and [46] is it known for certain that the $K_{S}^{0}$ was produced as a $K^{0}$. These eight measurements were obtained using a variety of incident particles $\left(1 K^{+}, 3 p\right.$, 


\begin{tabular}{|c|c|c|c|c|c|}
\hline Experiment & Reaction & Mass & Width & $\sigma^{\prime} s$ & Reference \\
\hline $\operatorname{LEPS}(1)^{*}$ & $\gamma^{12} C \rightarrow K^{+} K^{-} X$ & $1540 \pm 10$ & $<25$ & $\overline{4.6}$ & {$[29],[37]$} \\
\hline $\operatorname{LEPS}(2)^{*}$ & $\gamma d \rightarrow K^{+} K^{-} X$ & & & & {$[38]$} \\
\hline CLAS(d)* & $\gamma d \rightarrow K^{+} K^{-}(n) p$ & $1542 \pm 5$ & $<21$ & 5.2 & {$[40]$} \\
\hline $\operatorname{CLAS}(\mathrm{p})^{*}$ & $\gamma p \rightarrow K^{+} K^{-} \pi^{+}(n)$ & $1555 \pm 10$ & $<26$ & 7.8 & {$[41]$} \\
\hline SAPHIR* & $\gamma p \rightarrow \bar{K}^{0} K^{+}(n)$ & $1540 \pm 6$ & $<25$ & 4.8 & {$[42]$} \\
\hline COSY & $p p \rightarrow \Sigma^{+} K^{0} p$ & $1530 \pm 5$ & $<18$ & $4-6$ & [43] \\
\hline JINR & $p\left(C_{3} H_{8}\right) \rightarrow K_{S}^{0} p X$ & $1540 \pm 8$ & $9.2 \pm 1.8$ & 5.5 & {$[44]$} \\
\hline SVD & $p A \rightarrow K_{S}^{0} p X \mathrm{~A}=(\mathrm{C}, \mathrm{Si}, \mathrm{Pb})$ & $1526 \pm 4$ & $<24$ & 5.6 & {$[45]$} \\
\hline DIANA & $K^{+} X e \rightarrow K^{0} p(X e)^{\prime}$ & $1539 \pm 2$ & $<9$ & 4.4 & {$[46]$} \\
\hline$\nu \mathrm{BC}(\mathrm{ITEP})$ & $\nu N e \rightarrow K_{S}^{0} p X$ & $1533 \pm 5$ & $<20$ & 6.7 & {$[47]$} \\
\hline NOMAD & $\nu_{\mu} A \rightarrow K_{S}^{0} p X \mathrm{~A}=(\mathrm{Fe}, \mathrm{Al}, \mathrm{Pb})$ & $1528.7 \pm 2.5$ & $<21$ & 4.4 & {$[48]$} \\
\hline HERMES* & $e^{+} d \rightarrow K_{S}^{0} p X$ & $1528 \pm 3$ & $13 \pm 9$ & $\sim 5$ & [49] \\
\hline ZEUS* & $e^{-} p \rightarrow e K_{S}^{0} p X$ & $1522 \pm 3$ & $8 \pm 4$ & $\sim 5$ & {$[50]$} \\
\hline
\end{tabular}

Table 1.1: The results from experiments reporting the observation of the $\Theta_{5}(1540)^{+}$in a $K^{+} n$ or $K_{S}^{0} p$ invariant mass distribution. Seven of these experiments involve real or virtual photoproduction reactions [denoted by $*$, and four more involve hadroproduction on a hydrogen or nuclear target.

2 neutrino and $2 e$ ) and targets, and six of them are from inclusive production processes. These measurements yield an average signal mass $1530.8 \pm 2.2 \mathrm{MeV} / c^{2}$ for the $K_{S}^{0} p$ mode, and a somewhat lower upper limit estimate for the width $(<9 \mathrm{MeV})$. There is a discrepancy of $\sim 3.2 \sigma$ between the mass values from the $K^{+} n$ and $K_{S}^{0} p$ systems.

The overall mean value of the mass is $1535.3 \pm 2.6 \mathrm{MeV} / c^{2}$, where the error is estimated from the spread in the individual values, since this is larger than would be expected from the quoted errors on the measurements.

If the $\Theta_{5}(1540)^{+}$exists, its effects should be seen in $K^{+} d$ scattering data for incident $K^{+}$Lab. momenta around $440 \mathrm{MeV} / c$, unless its width is very small. Since the c.m. energy in this region is below pion production threshold, the cross section reaches the unitarity limit at resonance, and for spin $1 / 2$ is $B_{i} \times B_{f} \times 68 \mathrm{mb}$, where $B_{i}$ and $B_{f}$ are the branching fractions to the initial and final states. Integrating over the resonance peak, the net effect results in a contribution $\Gamma \times\left(B_{i} \times B_{f} \times 107 \mathrm{mb}\right)$, where $\Gamma$ is the width of the resonance. Several studies of the rather sparse scattering data in this region $[51,52,53,54]$ conclude that $\Gamma$ must be less than $5 \mathrm{MeV}$. In addition, in ref. [54] the Xe bubble chamber data of ref. [46], when interpreted in terms of $K^{+} n$ charge exchange scattering, yield the value $\Gamma=0.9 \pm 0.3 \mathrm{MeV}$, with no estimate of systematic uncertainty. The conclusion then is that if the $\Theta_{5}$ exists its width must be $<5 \mathrm{MeV}$, and may even be as small as $\sim 1 \mathrm{MeV}$. The width estimates of Table 1.1 are consistent with such values.

Since the distributions of fig. 1.2 are obtained in many different contexts, it is difficult to believe that the signals might be spurious. However, the fact that the mass value estimates are spread over a range which seems too large for the uncertainties quoted, and the observation that in all of the distributions of fig.1.2 the peak signal bin contains only $\sim 20-50$ events above background, indicate the need to exercise caution, especially in 
light of the recent results from JLab. [55] in what is essentially a much higher statistics repetition of the SAPHIR experiment (see section 1.5 w.r.t. the results from ref. [42]).

Cross section estimates for $\Theta_{5}$ production are either non-existent, unclear or unreliable. The COSY experiment [43] quotes a cross section of $0.4 \pm 0.1 \pm 0.1 \mu \mathrm{b}$, but does not indicate clearly whether all branching fraction values and isospin Clebsch-Gordan coefficients have been taken into account. The SAPHIR experiment [42] initially quoted a cross section for the reaction $\gamma p \rightarrow \bar{K}^{0} \Theta_{5}^{+}$of $300 \mathrm{nb}$, but this has since been reduced to $50 \mathrm{nb}$, and the recent JLab. measurements of the same reaction [55] yield a 95\% C.L. upper limit in the range 1-4 $\mathrm{nb}$ for the relevant region of photon lab. energy. To date, there is no other useful information.

\subsubsection{The $\Xi_{5}(1862)$}

This is the state contributing the baseline to the decuplet triangle of fig.1.1. Only one observation has been claimed to date [30]. This is from the NA49 Collaboration studying the interactions of a $158 \mathrm{GeV} / c$ proton beam in a liquid hydrogen target. The combined invariant mass distributions for the systems $\Xi^{-} \pi^{-}, \Xi^{-} \pi^{+}$and their anti-particle counterparts reveal a narrow signal of $\sim 68$ events over a background of $\sim 77$ events; the width is consistent with the detector resolution $\left(18 \mathrm{MeV} / \mathrm{c}^{2}\right)$. The fitted mass value is $1.862 \pm 0.002 \mathrm{GeV} / c^{2}$. A $\Xi^{--}$baryon is manifestly exotic; it occupies the lower-left vertex of the decuplet triangle of fig.1.1 with the minimal quark composition shown. It is troubling that there have been no other observations of this state to date (see section 1.5), but even more troubling that members of the same collaboration have publicly questioned the analysis [56].

\subsubsection{The $\Theta_{c}(3100)$}

Again there is only one experiment claiming evidence for this anti-charm baryon state [57]. The signal is observed by the H1 Collaboration at HERA in the $D^{*-} p$ and $D^{*+} \bar{p}$ invariant mass distributions; the fitted mass value is $3.099 \pm 0.003 \pm 0.005 \mathrm{GeV} / c^{2}$ and the width is consistent with detector resolution $\left(\sim 12 \mathrm{MeV} / c^{2}\right)$. The minimal quark content is $u u d d \bar{c}$, so that the state is a manifestly exotic pentaquark candidate. There has been no corroboration of this state to date; in particular, the ZEUS experiment operating under the same conditions at HERA has found no evidence of a signal.

\subsection{Negative Searches}

The experiments which have searched in vain for evidence of the three pentaquark states discussed in section 1.4 are summarized in Table 1.2, which has also been abstracted from ref. [39]. There is a fairly detailed discussion of the various non-observations in [39], and so it would seem unwarranted to repeat this here. Instead, a few additional remarks are included in the following: 
Table 1.2: Recent negative searches for pentaquark states. For each pentaquark state $(P)$ a - indicates that the state was not included in the search, while $\Downarrow$ indicates that the state was searched for and not observed, and $\Uparrow$ indicates that the state was searched for and observed.

\begin{tabular}{|l|l|lll|c|}
\hline Experiment & Search Reaction & $\theta^{+}$ & $\Xi_{5}$ & $\theta_{c}$ & Reference \\
\hline ALEPH & Hadronic Z decays & $\Downarrow$ & $\Downarrow$ & $\Downarrow$ & {$[58]$} \\
BABAR & $e^{+} e^{-} \rightarrow P X$ & $\Downarrow$ & $\Downarrow$ & - & {$[59]$} \\
BELLE & $K N \rightarrow P X$ & $\Downarrow$ & - & $\Downarrow$ & {$[60]$} \\
BES & $e^{+} e^{-} \rightarrow J / \psi(\psi(2 S) \rightarrow \theta \bar{\theta}$ & $\Downarrow$ & - & $\Downarrow$ & {$[61]$} \\
CDF & $p \bar{p} \rightarrow P X$ & $\Downarrow$ & $\Downarrow$ & $\Downarrow$ & {$[62]$} \\
COMPASS & $\mu^{+}\left({ }^{6} L i D\right) \rightarrow P X$ & $\Downarrow$ & $\Downarrow$ & - & {$[63]$} \\
DELPHI & Hadronic Z decays & $\Downarrow$ & - & - & {$[64]$} \\
E690 & $p p \rightarrow P X$ & $\Downarrow$ & $\Downarrow$ & - & {$[65]$} \\
FOCUS & $\gamma p \rightarrow P X$ & $\Downarrow$ & $\Downarrow$ & $\Downarrow$ & {$[66]$} \\
HERA-B & $p A \rightarrow P X$ & $\Downarrow$ & $\Downarrow$ & - & {$[67]$} \\
HyperCP & $\left(\pi^{+}, K^{+}, p\right) C u \rightarrow P X$ & $\Downarrow$ & - & - & {$[68]$} \\
LASS & $K^{+} p \rightarrow K K^{+} n \pi^{+}$ & $\Downarrow$ & - & - & {$[26]$} \\
L3 & $\gamma \gamma \rightarrow \theta \bar{\theta}$ & $\Downarrow$ & - & - & {$[64,69]$} \\
PHENIX & $A u A u \rightarrow P X$ & $\Downarrow$ & - & - & {$[70]$} \\
SELEX & $(\pi, p, \Sigma) p \rightarrow P X$ & $\Downarrow$ & - & - & {$[71]$} \\
SPHINX & $p C(N) \rightarrow \theta^{+} C(N)$ & $\Downarrow$ & - & - & {$[72]$} \\
WA89 & $\Sigma^{-} N \rightarrow P X$ & - & $\Downarrow$ & - & {$[74]$} \\
ZEUS & $e p \rightarrow P X$ & $\Uparrow$ & $\Downarrow$ & $\Downarrow$ & {$[50,75,76]$} \\
\hline
\end{tabular}

1. Almost all of the non-observations involve inclusive searches; the LASS analysis [26] is an exception, as are the old bubble chamber results (e.g. [27], [28]), which are not included in Table 1.2, but which give more restrictive cross section limits since they correspond to much lower incident beam momenta (the LASS data were obtained with an $11 \mathrm{GeV} / c K^{+}$beam).

In contrast, seven of the claimed observations result from exclusive production reactions for which combinatoric background is much less severe, or non-existent [46].

2. The SAPHIR experiment [42] is listed in Table 1.1 as one which observes a $\Theta_{5}$ signal in the exclusive reaction $\gamma p \rightarrow \bar{K}^{0} K^{+} n$ for photons in the lab. energy range $\sim 1.5-2.6 \mathrm{GeV}$. The resulting $K^{+} n$ invariant mass distribution is shown in fig.1.2, and seems to have much the same content as the other distributions in this figure. At the recent APS Meeting in Tampa, results from CLAS on the same reaction for photon energy 1.6-3.8 GeV were presented [55]. This new experiment is denoted as $\operatorname{CLAS}(p, 2)$; the previous CLAS experiment on hydrogen, denoted by $\operatorname{CLAS}(p)$ in Table 1.1, used higher energy photons to study a different reaction [41]. For the $\operatorname{CLAS}(p, 2)$ experiment, a great deal of time and effort went into calibrating the photon tagging system and the detector itself. The mass scale was carefully studied by means of several different photoproduction processes, and the understanding of the integrated luminosity verified by comparing the measured cross section values for 


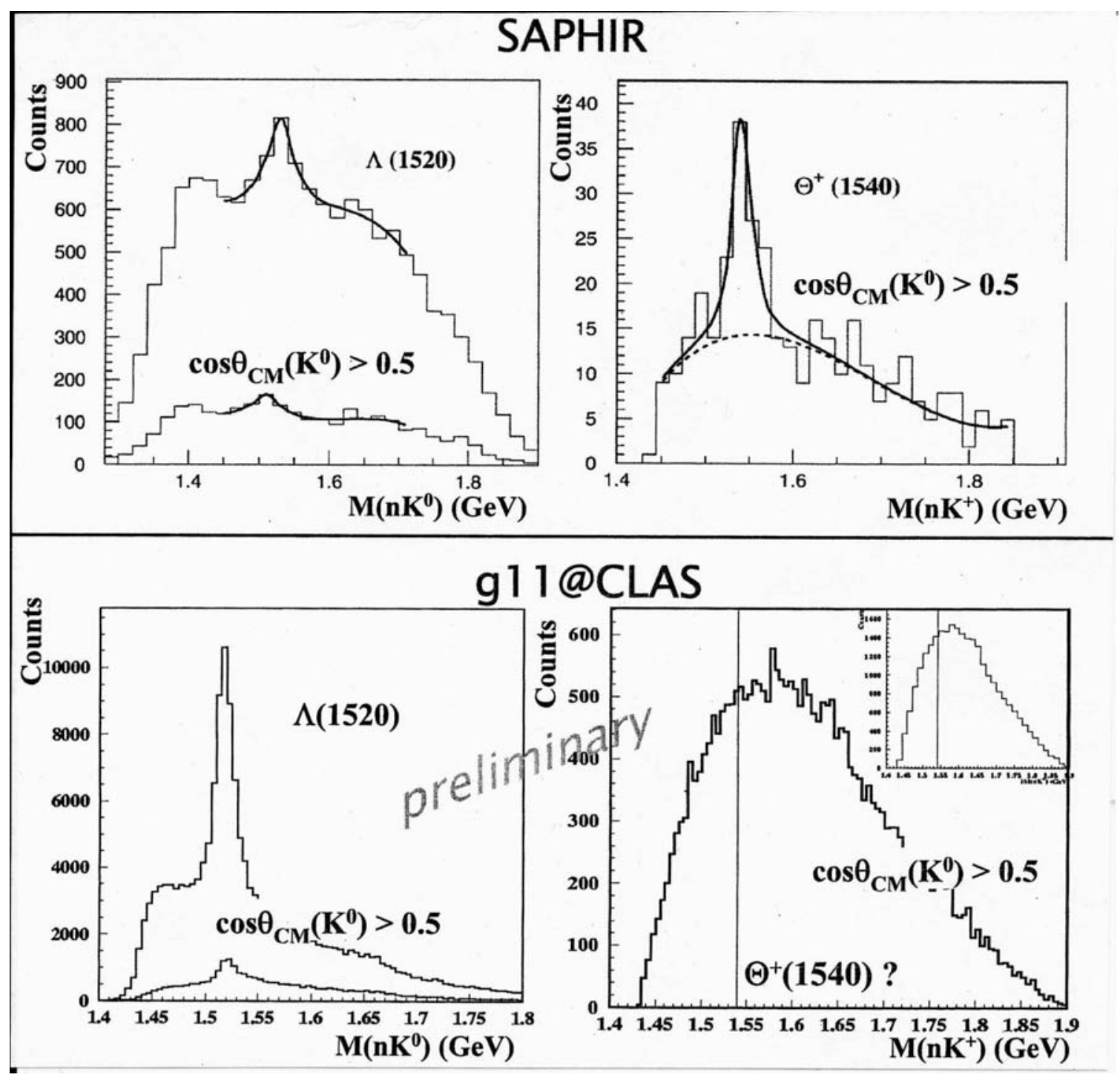

Figure 1.3: Comparison of the CLAS $(p, 2)$ results [55] to those from SAPHIR for the same reaction [42]. (Note that " $K^{0}$ " should be " $\bar{K}^{0}$ " everywhere in the figure)

different reactions with those obtained in other experiments, including SAPHIR. Finally, the experiment accumulated far more data than did the SAPHIR experiment.

The CLAS $(p, 2)$ results are shown in fig.1.3 in comparison to those from SAPHIR (the $K^{0}$ 's in this figure should be $\bar{K}^{0}$ 's). The plots on the left compare the $n \bar{K}^{0}$ distributions, and show how the requirement that the $\bar{K}^{0}$ be forward in the c.m. reduces the $\Lambda(1520)$ signal, (Note that for some strange reason the SAPHIR distribution extends well below $n \bar{K}^{0}$ threshold!). The plots on the right compare the $n K^{+}$mass distributions after requiring a forward $\bar{K}^{0}$. The CLAS data are plotted in $5 \mathrm{MeV} / c^{2}$ bins, while those of SAPHIR are in bins of $\sim 14.6 \mathrm{MeV} / c^{2}$, so that at the position of the $\Theta_{5}$ the density $d N / d m$ is $\sim 40$ times greater for CLAS, i.e. the SAPHIR peak would register at about the middle of the first $y$-axis tick-mark in the 
CLAS plot. There is no evidence of a signal in the CLAS data (the mass resolution is $\sim 15 \mathrm{MeV} / c^{2}, \mathrm{FWHM}$ ), and $95 \%$ C.L. limits on the $\Theta_{5}$ production cross section range from $\sim 4 \mathrm{nb}$ at threshold to $\sim 1 \mathrm{nb}$ at a photon energy of $3.8 \mathrm{GeV}$. The original SAPHIR $\Theta_{5}$ cross section estimate was $300 \mathrm{nb}$ [42], and a new estimate puts this now at $\sim 50 \mathrm{nb}$.

The original claim that there was significant $(4.8 \sigma !)$ evidence of $\Theta_{5}$ production in this reaction is clearly untenable in the face of this new $\operatorname{CLAS}(p, 2)$ result. This demonstrates very clearly the importance of verifying such controversial results as those claiming to show evidence of pentaquark production at the highest statistical level possible in the context of a well-calibrated detector (see further discussion in section 1.6).

The fact that the SAPHIR measurement should now be excluded immediately calls into question the remaining claims associated with the other distributions in fig.1.2, since these are uncomfortably close to SAPHIR in statistical content.

3. The $B A B A R$ search for $\Theta_{5}(1540)$ production in $e^{+} e^{-}$interactions which is described in Chapter 3 of this thesis is Incorporated in ref. [59], and has been presented at ICHEP, Beijing (2004) and at other conferences. These results are included in Table 1.2 ; note that an unsuccessful search for the $\Xi_{5}$ is described also in [59].

4. The BABAR search for the $\Theta_{5}(1540)$ in electroproduction and hadroproduction interactions in the material of the inner detector, which is described in Chapter 4 of this thesis, should eventually be included in Table 1.2. The results have been presented already at Moriond QCD 2005, at the Dublin IOP Meeting, at the Tampa APS Meeting and at DIS2005 in Madison, Wisconsin.

\subsection{Present Status and Future Outlook}

There has been a great deal of activity of an experimental and theoretical nature since late 2002 and early 2003 when the LEPS(1) [29, 37] result became public. The subsequent claims for $\Theta_{5}(1540)$ observations are summarized in Table 1.1, and since these are obtained in a rather diverse group of experimental contexts, it would appear difficult to deny the existence of the $\Theta_{5}$, especially with the NA49 claim for the existence of the exotic $\Xi_{5}^{--}$ anti-decuplet partner [30]. However, as time has gone on, this latter state has not been observed in any other experiment, and in fact there is even debate within the NA49 collaboration as to the validity of the analysis [56]. In addition, several other experiments have searched for the $\Theta_{5}$ with large statistics and excellent mass resolution and have found no signal [see Table 1.2]; the observations and non-observations have been reviewed in a

fairly even-handed way by Dzierba et al. in ref. [39], and by G. Trilling in [28]. The present situation can best be summarized perhaps by saying that the jury is still out. Indeed, in a recent SLAC talk from K. Hicks he expressed a certain level of pessimism as 
to the existence of the $\Theta_{5}$, this from someone who has been deeply involved in both LEPS analyses [29, 37, 38] as well as the CLAS deuterium analysis [40].

At the end of G. Trilling's article in PDG2004 [28], in considering the type of experiment required in order to establish fully the existence of the $\Theta_{5}$, he suggests the need for:

"Confirmation with high statistics, much-improved effective mass resolution, and excellent particle identification, of the photoproduction results...";

by implication it follows that such studies are also required if the existence is to be disproved or at least questioned seriously. In fact, data meeting these requirements (although without particularly good mass resolution) were presented recently at the APS Tampa Meeting [55]. These results are from a high-statistics CLAS experiment with a liquid hydrogen target which essentially repeats the SAPHIR study [42] of the reaction $\gamma p \rightarrow \bar{K}^{0} K^{+} n$. With a factor $\sim 40$ increase in statistics in the $\Theta_{5}$ mass region, no signal is observed, and cross section upper limits $\sim 1-4 \mathrm{nb}$ are established. The SAPHIR experiment was the first to claim confirmation of the original observation. The CLAS result demonstrates quite clearly that such a claim cannot be maintained in the face of the new high-statistics result.

The BABAR analysis of electroproduction [or quasi-real photoproduction] data presented in Chapter 4 of this thesis also satisfies Trilling's criteria when compared to the HERMES and ZEUS analyses claiming observation of the $\Theta_{5}$. The data sample is large [ 200 times the HERMES quasi-real photproduction data, $\sim 14$ times ZEUS data for $Q^{2}>$ $20 \mathrm{GeV}^{2}$, the mass resolution in the $\Theta_{5}$ region is excellent [ $\sim 3$ times better than HERMES, and slightly better than ZEUS], and particle-identification capability is very good [HERMES has no low-momentum proton identification capability, the BABAR $d E / d x$ resolution is better than that of ZEUS, the proton sample is of higher purity, and the Cherenkov detector can be used to help identify associated particles which ZEUS does not attempt (other than for the scattered lepton)]. The BABAR results described in Chapter 4 show no evidence for $\Theta_{5}$ production, and the comparisons with HERMES and ZEUS suggest that their claims of observation are less than convincing.

It follows that the new CLAS and BABAR high statistics analyses do not confirm three of the claims summarized in Table 1.1, and so although "the jury is still out" it would appear that it might be beginning to tilt toward a guilty verdict!

However, a great deal of future experimental investigation will be required in order to provide a definitive answer to the question of the existence of pentaquark states. At JLab, the high-statistics results from the CLAS photoproduction experiment on deuterium may be available by the summer. A large statistics run at COSY to investigate further $p p \rightarrow \Sigma^{+} p K_{S}^{0}$ is approved, and at JLab an experiment to investigate $5.7 \mathrm{GeV}$ electroproduction on deuterium in CLAS has now finished data-taking. The latter experiment has as a primary goal a search for the production of the $\Xi_{5}^{--}$state. An electroproduction experiment on deuterium is approved for Hall A at JLab for a three-week run early in 
2006. This involves a high-resolution forward spectrometer to detect $K^{-}$mesons in the 3 $\mathrm{GeV} / c$ region, a low-momentum spectrometer to detect and identify $K^{+}$mesons in the 0.5 $\mathrm{GeV} / c$ region, and a large neutron counter to measure neutrons in the $0.5 \mathrm{GeV} / c$ range. The mass resolution in the $\Theta_{5}$ region is estimated to be $\sim 1 \mathrm{MeV} / c^{2}[\mathrm{HWHM}]$. The $K^{+}$and neutron momenta match very well to the regions in which the BABAR electroproduction experiment detects $K_{S}^{0}$ mesons and protons, and it will be very interesting to compare the JLab results with those described in Chapter 4 of this thesis.

In summary, the high levels of interest and activity generated in recent years by the possible existence of pentaquark states promise to be maintained in the immediate future. It would seem that the weight of experimental evidence is beginning to come down on the side of non-existence, and it is to be hoped that new high-statistics results to become available within the next year will lead to a definitive answer to this question.

\subsection{Motivation and Thesis Outline}

The summary provided in section 1.6 expresses the high level of interest associated with the topic of Pentaquarks, both in the recent past and in the immediate future. As discussed there, resolution of the issue of existence requires relevant information from experiments providing large data samples of high quality. The BABAR experiment is an excellent source of such data samples, both from the conventional $e^{+} e^{-}$interactions in the PEP II Collider, and from interactions in the material of the BABAR detector.

A study of inclusive $K_{S}^{0}$ production in $B A B A R e^{+} e^{-}$collisions revealed that more than one million signal $K_{S}^{0}$ candidates were reconstructed per $\mathrm{fb}^{-1}$ of integrated luminosity. In addition, there was copious production of protons and anti-protons, and so it was quite clear that the BABAR data sample would be an extremely rich source for the production of $K_{S}^{0} p$ [and $\bar{p}$ ] systems in the low-mass region relevant to a search for $\Theta_{5}$ production. The corresponding particle identification information is of very high quality, and the invariant mass resolution proved to be $\sim 2 \mathrm{MeV} / \mathrm{c}^{2}$ (HWHM), and so is well-suited to a search for the production of a state of very small width. For these reasons it was decided to carry out the search for the production of the $\Theta_{5}$ in $e^{+} e^{-}$collisions which is described in Chapter 3 .

In the course of the inclusive $K_{S}^{0}$ studies, it was realized that there was significant $K_{S}^{0}$ production from the PEP II beampipe in the horizontal plane. A previous study of interactions in the beampipe yielding protons had been performed in order to use the nominal dimensions of the beampipe to check the radial and longitudinal length scales of the BABAR coordinate system [77]. It then became clear that the entire Run 1-4 BABAR data set could provide high-statistics, high-resolution data on the production of the $K_{S}^{0} p$ system as a result of interactions in the material of the inner BABAR detector and the PEP II beampipe. Such events are due to the secondary interactions of hadrons produced in primary $e^{+} e^{-}$collisions, to particles contributing to the general BABAR background, but also to the interactions of beam-halo electrons and positrons in the beampipe material in 
the horizontal plane. These latter interactions are the result of the presence of vertical permanent magnet dipoles close to $(\sim 20 \mathrm{~cm})$ the interaction region whose purpose is to bend the electron and positron beams into head-on collision; this is a feature unique to PEP II. Seven of the thirteen claims for $\Theta_{5}$ observation listed in Table 1.1 result from real or virtual photoproduction (although the SAPHIR claim should be considered negated by the new CLAS(p, 2) measurement), and so it was quite clear that the results of the highstatistics electroproduction experiment which BABAR could contribute in this somewhat unconventional way might be able to make a useful contribution to the ongoing debate concerning the existence of the $\Theta_{5}$. At the same time, the events from the entire inner detector provided an even larger sample of high-quality data for use in defining the mass and width of the $\Theta_{5}$ should a signal be in fact observed. This is the motivation for the study described in Chapter 4 of the thesis.

The PEP II Asymmetric-energy Collider, and its performance from the start of BFactory data-taking in May, 1999 until the end of Run 4, in June, 2004 are described briefly in Chapter 2. An overview of the BABAR Detector, and brief descriptions of the structure and performance of each detector sub-system are included also in this chapter.

The search for inclusive production of the $\Theta_{5}(1540)^{+}$, with decay to the $K_{S}^{0} p$ final state, in $e^{+} e^{-}$collisions at a c.m. energy of $\sim 10.58 \mathrm{GeV}$ is then described in detail in Chapter 3. No signal is observed, and upper limits on the inclusive cross section as a function of c.m. momentum, $p^{*}$, are obtained. The corresponding limits on the total cross section are compared to those for other meson and baryon states, and are found to be significantly lower than would be expected for a baryon in the mass region $\sim 1.54 \mathrm{GeV} / c^{2}$.

In Chapter 4 it is demonstrated that interactions in the material of the inner $B A B A R$ detector leading to the production of the $K_{S}^{0} p$ system can be found in large numbers for the complete Run 1-4 BABAR data sample. As mentioned above, such events are due to secondary hadrons from primary $e^{+} e^{-}$collisions, to particles contributing to the general background in the detector, and, most importantly, to beam-halo electrons and positrons. The latter enable high-statistics, high-resolution searches for electroproduction of the $\Theta_{5}$ in the beryllium of the BABAR beampipe and in the tantalum foils wrapped around the ends of the beampipe in order to reduce detector background. The entire data sample from the inner detector permits a search aimed simply at establishing the existence of the $\Theta_{5}$ and measuring its mass and width to high precision. Neither search yields evidence of a signal, even when additional selection criteria aimed at increasing the probability of observation are incorporated. Finally, comparisons are made between the results of the $e^{-}$Be search and those of other electroproduction experiments in which observation of a $\Theta_{5}$ signal has been claimed.

The conclusions drawn from the studies presented in this thesis are summarized in Chapter 5, and the Appendices contain additional, but rather repetitive, material related to Chapter 4, which is included only for purposes of completeness. 


\section{Chapter 2}

\section{The BABAR detector}

This chapter serves as an overview of the BABAR detector that is situated at the SLAC $B$ Factory, highlighting some of the main motivations for its present architecture and design. Primarily intended for the systematic study of $C P$ violating asymmetries in the decay of $B$ mesons to $C P$ eigenstates, the design of all the components of the detector is optimized for those studies. However it is also well suited to, and has the capability to contribute to, precision spectroscopy and other physics topics such as precise measurements of the decay of charm mesons and $\tau$ leptons, along with the search for "new" and rare processes that become accessible with high luminosity.

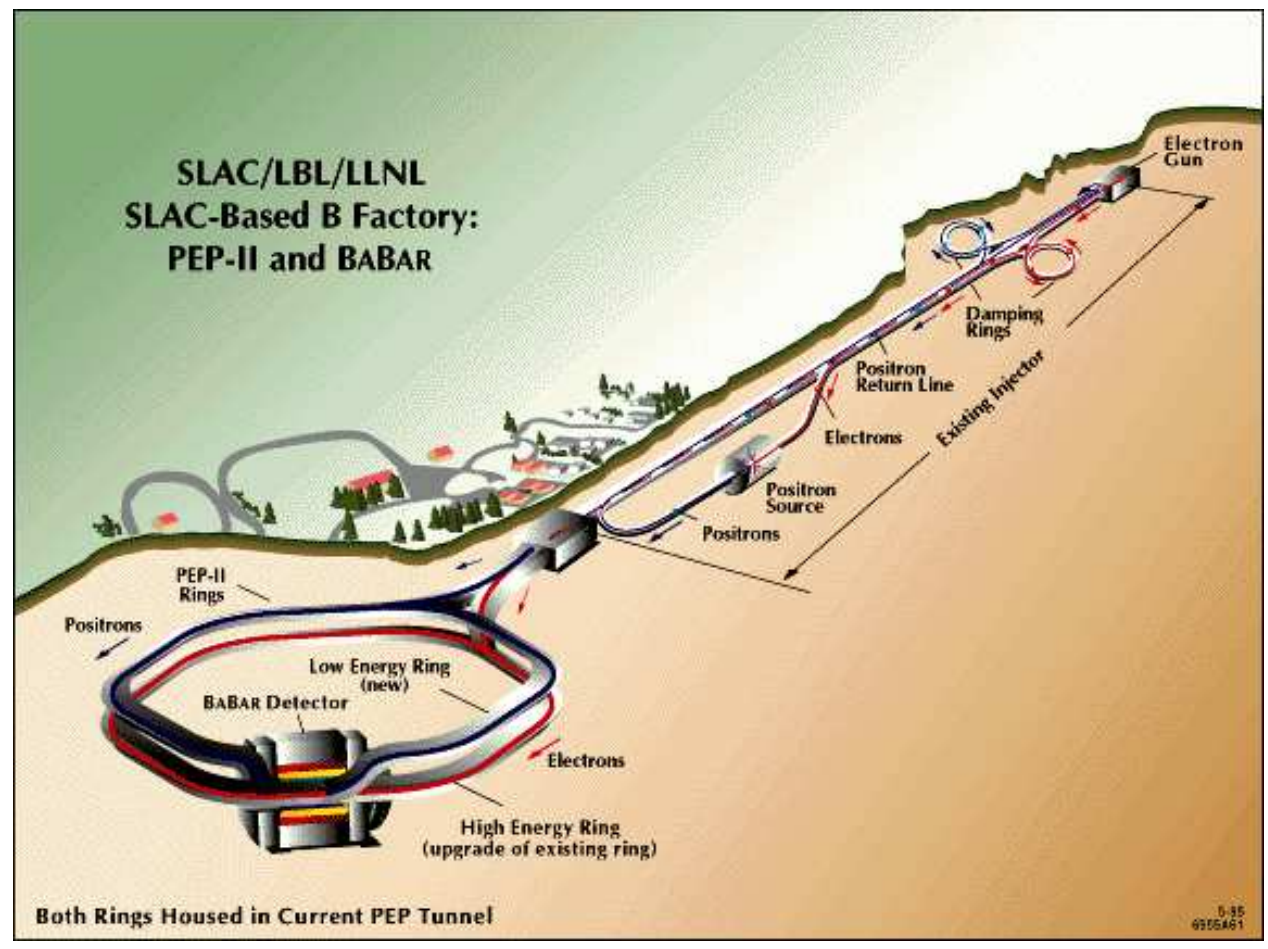

Figure 2.1: The above figure, shows a picture of the PEP-II storage ring facility; bunches are accelerated in the pre-existing SLAC LINAC, injected into the storage rings and collide inside the BABAR detector.

The beam facility at SLAC that is used in the B-factory experiment is the PEP-II 
asymmetric $e^{+} e^{-}$collider illustrated in fig. 2.1. It has already delivered a peak luminosity of $>9 \times 10^{33} \mathrm{~cm}^{-2} \mathrm{~s}^{-1}$ well in excess of the design $3 \times 10^{33} \mathrm{~cm}^{-2} \mathrm{~s}^{-1}$, and possible upgrades to even higher luminosities are under discussion. Operating at a centre-of-mass energy of $10.58 \mathrm{GeV}$ allows for the production of $B$ meson pairs from $\Upsilon(4 S)$ decays. In PEP-II, $9 \mathrm{GeV}$ electrons collide head on with $3.1 \mathrm{GeV}$ positrons, resulting in a Lorentz boost to the $\Upsilon(4 S)$ resonance of $\beta \gamma=0.56$. This enables the reconstruction of the separated decay vertices of the two $B$ mesons, allowing the determination of their relative decay times and the measurement of the time dependence of $B^{0} \bar{B}^{0}$ oscillations [78]. This requires events where one $B$ meson decaying to a $C P$ eigenstate is fully reconstructed (typically two or more charged particles and one or two $\pi^{0}$ 's), and the other $B$ meson is tagged as a $B^{0}$ or $\bar{B}^{0}$ by its decay products: a charged lepton, kaon, or other flavor-identifying features. Combining these requirements with the branching ratio for $B$ mesons to $C P$ eigenstates of typically $10^{-3}-10^{-6}$, places stringent requirements on the detector, which should have [79]:

- a large and uniform acceptance down to small polar angles relative to the boost direction;

- excellent reconstruction efficiency for charged particles down to $60 \mathrm{MeV} / c$ and for photons to $20 \mathrm{MeV}$;

- very good momentum resolution to separate small signals from background;

- excellent energy and angular resolution for the detection of photons from $\pi^{0}$ and $\eta^{0}$ decays, and from radiative decays in the range from $20 \mathrm{MeV}$ to $4 \mathrm{GeV}$;

- very good vertex resolution, both transverse and parallel to the beam direction;

- efficient electron and muon identification, with low misidentification probabilities for hadrons. This feature is crucial for tagging the $B$ flavor, for the reconstruction of charmonium states, and is also important for the study of decays involving leptons;

- efficient and accurate identification of hadrons over a wide range of momenta for $B$ flavor-tagging, and for the reconstruction of exclusive states; modes such as $B^{0} \rightarrow$ $K^{ \pm} \pi^{\mp}$ or $B^{0} \rightarrow \pi^{+} \pi^{-}$, as well as charm meson and $\tau$ decays;

- a flexible, redundant, and selective trigger system;

- low-noise electronics and a reliable, high bandwidth data-acquisition and control system;

- detailed monitoring and automated calibration;

- an online computing and network system that can control, process, and store the expected high volume of data; and

- detector components that can tolerate significant radiation doses and operate reliably under high-background conditions. 


\subsection{Overview of the Detector}

The detector illustrated in fig. 2.2, is composed of several sub-detectors each of which is responsible for fulfilling one or more of the physics requirements outlined above.

The main components of the detector include a multi-layer silicon vertex tracker surrounded by a central cylindrical wire drift chamber, for the measurement of charged tracks. Electromagnetic showers from electrons and photons are detected in an array of CsI crystals located just inside the solenoidal coil of a superconducting magnet. Muons and neutral hadrons are identified by arrays of resistive plate chambers inserted into gaps in the steel flux return of the $1.5 \mathrm{~T}$ magnet. Charged hadrons are identified by $\mathrm{d} E / \mathrm{d} x$ measurements in the tracking detectors and by a ring imaging Cherenkov detector surrounding the drift chamber. The trigger, data acquisition and data monitoring systems are controlled by custom-designed online software. Apart from the DIRC, the detector was completed in Feb 1999 on time and within budget. The first collisions were recorded on May 26, 1999. The DIRC, which had experienced delays with quartz bar manufacture, was completed in the Fall of 1999, and physics quality data-taking began shortly thereafter.

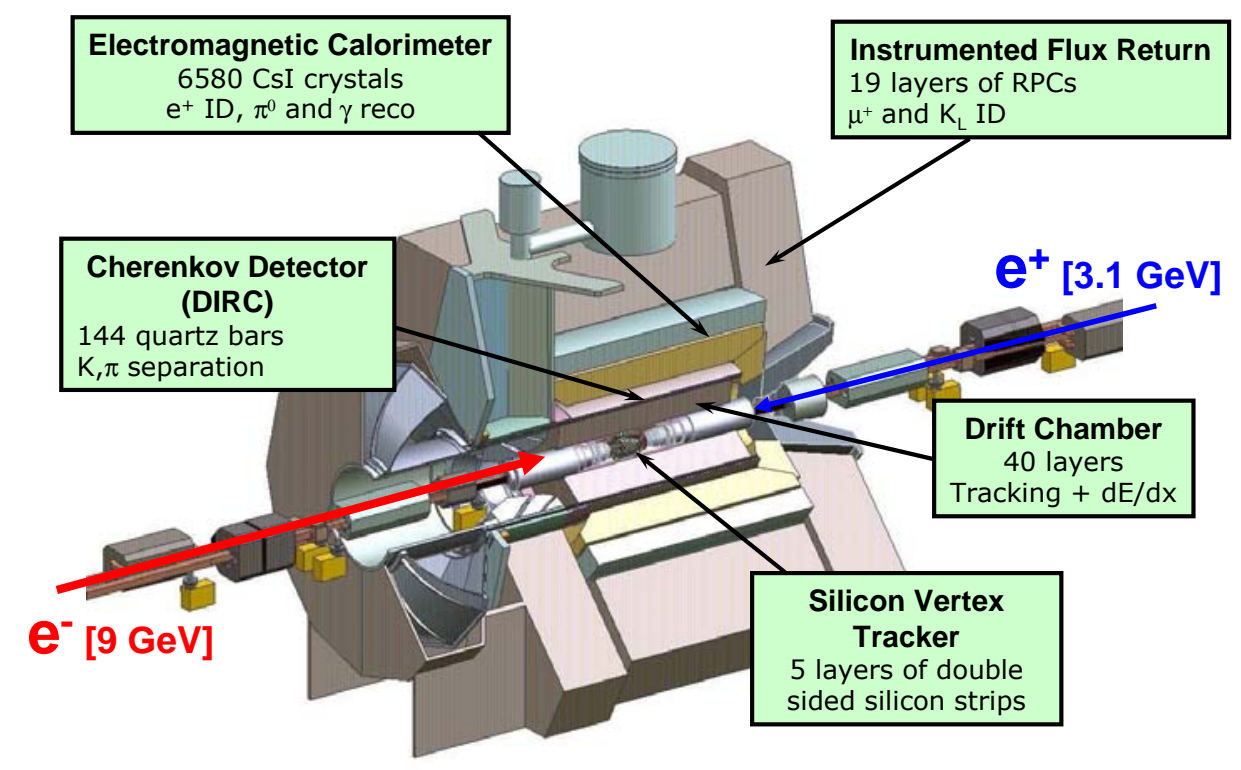

Figure 2.2: Shown is an overview of the BABAR detector. Starting at the interior and moving outward, the detector components shown are the Silicon Vertex Tracker (SVT), the Drift CHamber (DCH), the Detector of Internally Reflected Cherenkov Radiation (DIRC) particle identification system, the ElectroMagnetic Calorimeter(EMC), and the Instrumented Flux Return (IFR) housing the muon and neutral hadron detector. Some of the beamline magnets (namely, Q1, Q2 and Q4) near the interaction region are shown as they aid the production of the high luminosity required at PEP-II. 


\subsection{Silicon Vertex Tracker (SVT)}

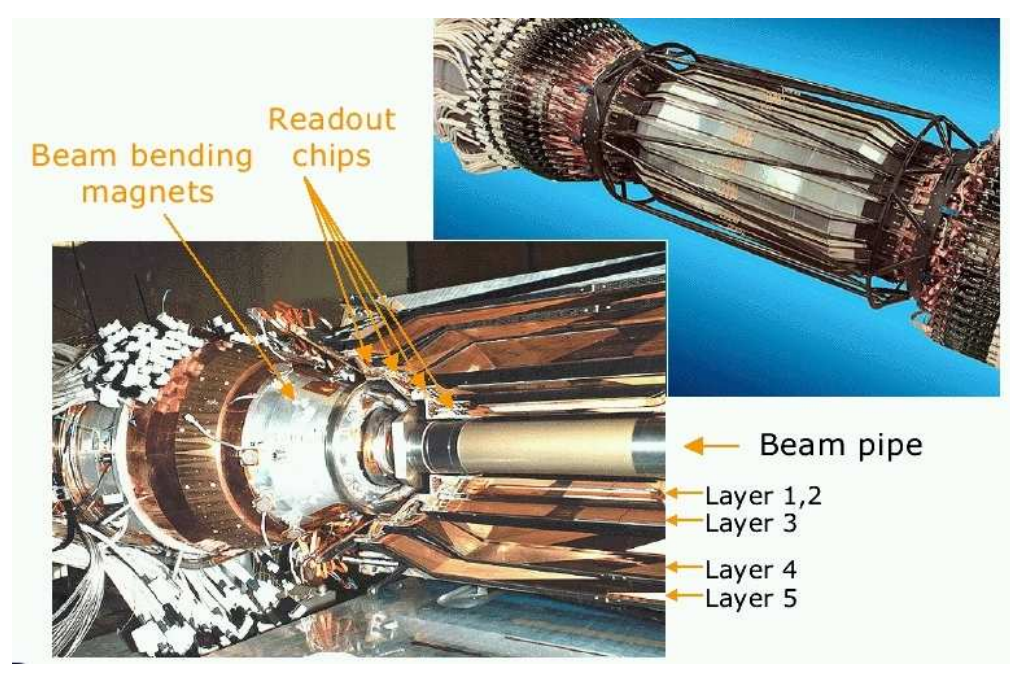

Figure 2.3: The partially assembled SVT, showing the positioning of the silicon wafers around the IP region, and the B1 bending magnets

The Silicon Vertex Tracker (SVT) was designed for very high precision measurement of azimuthal (phi) and longitudinal (z) coordinates on the trajectories of charged particles in the immediate vicinity of the PEP II beampipe. When combined with coordinate information from the Drift Chamber (DCH) (Section 2.3) in the BABAR charged track reconstruction software, the result is to produce very accurate information on position, direction and momentum for charged particles produced in PEP II $e^{+} e^{-}$collisions. In turn this yields the precision vertex reconstruction capability essential to the study of CP violation, which is the primary physics goal of the BABAR experiment.

The SVT consists of five layers of double-sided silicon micro-strip detectors; the silicon strips are pitched at $\sim 20-50$ microns, depending on function (i.e. whether measuring $\phi$ or z) and layer number. Layers 1-3 occupy a radial range from $\sim 3$ to $\sim 6 \mathrm{~cm}$, with each layer forming a hexagonal cylinder coaxial with the beampipe axis. Layers 4 and 5 are in the radial range from $\sim 12-\sim 15 \mathrm{~cm}$, in 16- and 18-sided polygon configurations with innovative lamp-shade structures fore and aft. The detector is divided vertically into $x>0$ and $x<0$ "clamshells", and fig. 2.3 shows the beampipe in the process of being captured by the $x>0$ clamshell. The second picture in fig. 2.3 shows the completed detector mounted between the B1 magnets to which the SVT carbon fibre support frame is attached. More details on the geometrical and mechanical structure are provided in Chapter 4, in particular in figs. 4.4 - 4.6, and fig.4.7 shows how the completed SVT and Support Tube which houses it are incorporated into the BABAR detector.

In addition to providing coordinate measurements, the SVT strip clusters associated with a charged particle track provide specific ionisation $(\mathrm{dE} / \mathrm{dx})$ information by means of time-over-threshold measurements. After calibration these are equivalent to measurements of energy deposition in the silicon as a result of ionisation of the medium. Since the number 
of samples used in calculating $\mathrm{dE} / \mathrm{dx}$ for a given track is quite small (typically $\sim 6$ ) the resolution is not particularly good ( $\sim 18 \%$ of $\mathrm{dE} / \mathrm{dx}$ ). Nevertheless, for the analysis described in Chapter 4 of this thesis the SVT dE/dx measurements do provide useful particle identification information, since the track momenta involved are quite low (see fig.4.28).

The decision to build a five-layer SVT was driven by the idea that the SVT system should be capable of performing stand-alone track- finding. The thinking was that three layers are needed to define a candidate helix, a fourth layer is required for corroboration, and a fifth layer is necessary in order to compensate for inefficiency etc. In practice, this actually works quite well, and pion tracks of transverse momenta from $\sim 100$ down to $\sim 50$ $\mathrm{MeV} / c$ can be found with quite good efficiency. Above $\sim 100 \mathrm{MeV} / c$, the DCH trackfinding takes over for the most part, although higher momentum tracks which interact or decay between the outer radius of the SVT and the inner radius of the drift chamber can still be found by the SVT track reconstruction software. This capability is particularly relevant for physics analyses involving $D^{*}(2010)$ production and decay to $D^{0} \pi^{ \pm}$, since the $\pi^{ \pm}$produced tend to have rather low lab. momentum.

The SVT stand-alone track-finding capability is also of great relevance to the so-called Global Alignment (GA) of the BABAR detector. In order to satisfy earthquake safety requiremnents, the Support Tube containing the SVT may not be attached directly to the DCH. However, it is subject to thermal, mechanical and magnetic stresses, the last because it contains the B1 and Q1 permanent magnet machine elements. This causes the position and orientation of the SVT to vary slightly over time w.r.t. the DCH, which defines the BABAR coordinate system. The SVT coordinate system is mapped into the DCH system by means of a set of GA parameters which define a rigid body translation and rotation. This set of six parameters is obtained for each run number during data- taking by matching an ensemble of charged tracks, each of which is reconstructed separately in the SVT and DCH coordinate frames, at the wall of the Support Tube. By means of these GA parameters, local SVT measurements are converted to the DCH coordinate system for use in the general track reconstruction software. This procedure is discussed further in Chapter 4, Section 4.5 in relation to the tomography of the SVT and Support Tube.

Finally, the z coordinate measurement precision in the SVT is at least an order of magnitude better than that which can be obtained from the $\mathrm{DCH}$ because of the small wire angle values in the stereo layers (Section 2.3). It follows that the dip angle (essentially $\mathrm{dz} / \mathrm{dr}$ ) for a charged track is defined almost entirely by the SVT z measurements on the track. This of course has important consequences for the total momentum of the track, but it also has a significant impact on the particle identification capability of the DIRC (Section 2.4). The latter is very sensitive to the position and direction in three dimensions of a charged particle trajectory at entry to the relevant quartz bar, and in this sense the coordinate measurements provided by the SVT play a crucial role in reliable particle identification as well as in precision vertexing. 


\subsection{Drift Chamber (DCH)}

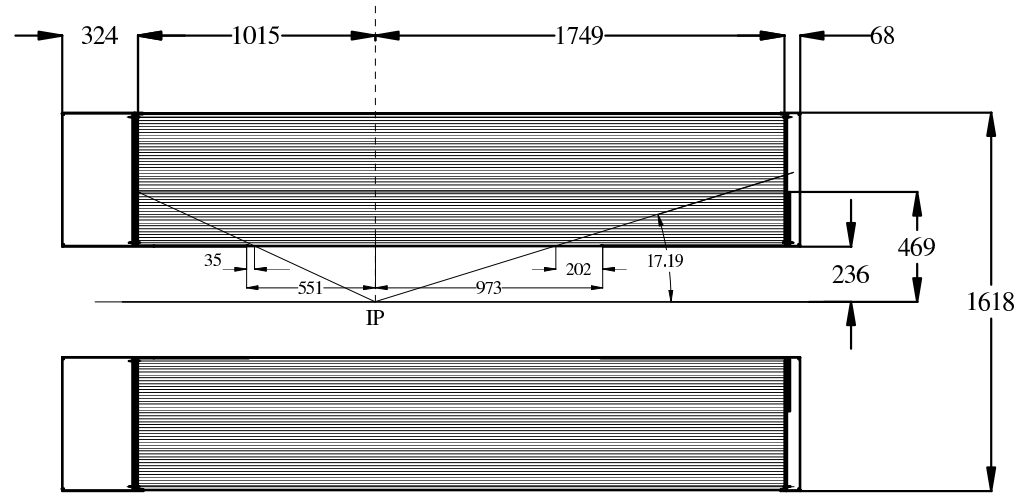

Figure 2.4: Drift Chamber Side View [79], dimensions in mm. The interaction point (IP) is not located in the center of the chamber, but rather is offset to the left in order to optimize C.M. acceptance, given the asymmetric beam energies.

The Drift Chamber (DCH) is the main charged particle tracking device in BABAR and as such must provide efficient pattern recognition capability and high precision transverse momentum $\left(\mathrm{p}_{T}\right)$ and position measurements over its large fiducial region. At the same time it is expected to yield corresponding measurements of specific ionisation $(\mathrm{dE} / \mathrm{dx})$ of sufficient quality as to contribute significantly to the process of charged particle identification in BABARSince the beginning of data-taking in 1999, the DCH has met all of these requirements at a consistently high level of performance, and distributions in quantities such as invariant mass, $\mathrm{dE} / \mathrm{dx}$, etc. remain virtually identical from the initial data-taking in 1999-2000 (Run 1) to that in 2003-2004 (Run 4). In addition, the DCH serves to define the BABAR coordinate system.

The DCH is a large cylindrical tracking volume of internal length $276.4 \mathrm{~cm}$ oriented within the cryostat of the BABAR superconducting magnet such that its axis coincides to a good approximation with the axis of the $\sim 1.5 \mathrm{~T}$ magnetic field. The inner cylinder, which can be seen in fig. 2.5 during DCH stringing at TRIUMF, consists of fore and aft sections of $\mathrm{Al}$ of $5 \mathrm{~mm}$ thickness, internal radius $23.6 \mathrm{~cm}$, and a low-mass central section of $\mathrm{Be}$ of thickness $1 \mathrm{~mm}$ and internal radius $23.8 \mathrm{~cm}$ whose length is defined by the tracking fiducial region. The inside and outside surfaces of the Be section are coated with BR127 anti-corrosion paint, which contains sodium chromate, hence the greenish colour visible in fig. 2.5. The $\mathrm{Al}$ endplates are $24 \mathrm{~mm}$ thick, but the forward endplate is thinned to $12 \mathrm{~mm}$ thickness beyond radius $\sim 47 \mathrm{~cm}$ in order to reduce the amount of material in front of the Electromagnetic Calorimeter (EMC) endcap (Section 2.5). All of the front-end electonics used to read out the DCH data is mounted on the rear endplate. The outer cylinder is composed of a hexagonal Nomex foam structure contained within carbon fibre skins, each of which is clad in $\mathrm{Al}$ foil in order to provide r.f. shielding. More details on the geometrical and mechanical structure of the DCH are provided in Chapter 4, Sections 4.3 and 4.5; in particular fig.4.7(d) shows schematically where the DCH is located within the structure 
of the BABAR detector.
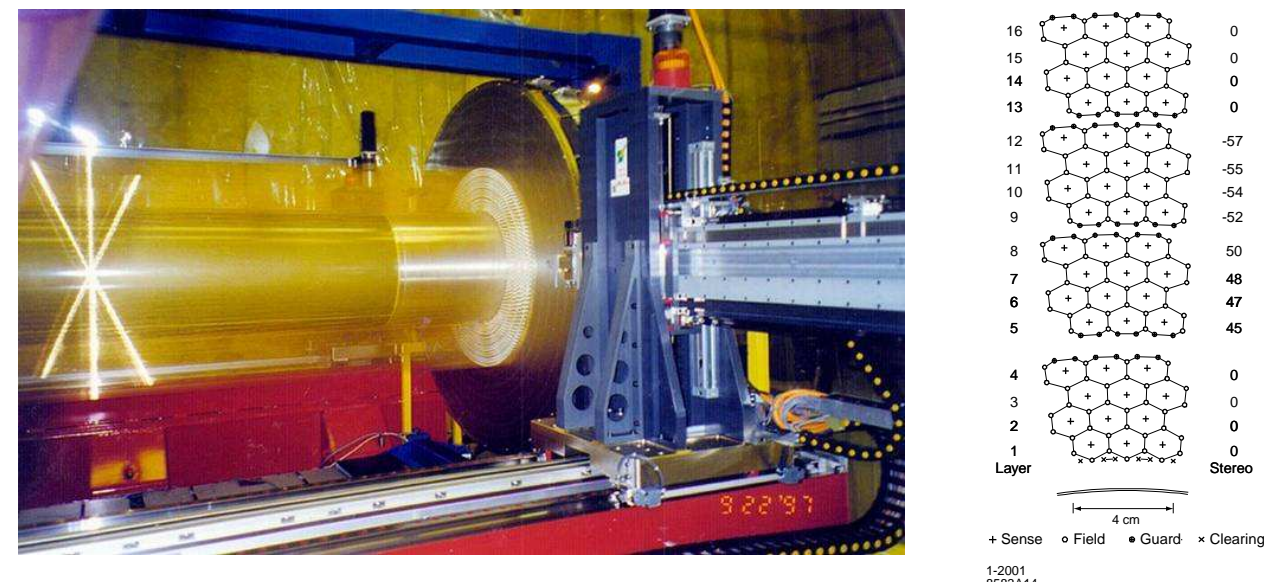

Figure 2.5: (Left)The Inner DCH during stringing, (Right) DCH Cell Design

The chamber is strung with 7104 gold-coated tungsten-rhenium sense wires of 20 micron diameter; the field and clearing wires are of gold-coated Al, 120 micron in diameter, while the guard wires have the same composition but are of diameter 80 micron; there are 28768 wires in total, and the stringing operation required 15 weeks. The sense wire pattern forms 40 approximately cylindrical layers (the stereo layers cannot define a cylindrical surface), with radially-sequential groups of four layers clustered to form 10 superlayers. The field wires are arranged to create the approximately hexagonal drift cell pattern illustrated in fig.2.5 for the four innermost superlayers; the lines connecting the field wires are drawn merely to illustrate the relevant drift cell boundaries. The individual cells have radial height $\sim 1.2 \mathrm{~cm}$ and azimuthal width $\sim 1.8-1.9 \mathrm{~cm}$, so that the maximum drift distance is $\sim 9 \mathrm{~mm}$, which corresponds to a drift time of $\sim 600$ nsec. The time-to-distance calibration then yields position resolution of $\sim 100$ microns over most of the drift cell, although this degrades to more than 200 microns close to the sense wire and near the cell boundary. The individual superlayers provide axial (A) or stereo (U or V) information. From the innermost superlayer (minimum radius $\sim 26 \mathrm{~cm}, 96$ cells per layer) to the outermost (maximum radius $\sim 78 \mathrm{~cm}, 256$ cells per layer) the sequence is AUVAUVAUVA, and the values of the stereo angle increase in magnitude from $\sim 2.6 \mathrm{deg}$. to $\sim 4.3 \mathrm{deg}$. with increasing radius.

The gas mixture used was chosen so as to minimize multiple scattering, and hence to optimize transverse momentum resolution. A mixture consisting of $80 \% \mathrm{He}$ and $20 \%$ isobutane by volume has satisfied this requirement while providing a level of performance which compares favourably to those obtained in the past for the more traditional argonbased gas mixtures. The DCH was designed to operate at a high-voltage value of $1960 \mathrm{~V}$ with no water vapour added to the gas mixture. However, after a near-disastrous highvoltage accident during commissioning, this was reduced to $1900 \mathrm{~V}$ for the period October, 1999 to July, 2000. During this time, a loss of track-finding efficiency was observed for tracks at near normal incidence to the DCH sense wires, for which saturation effects are 
maximal. The high-voltage was increased therefore to $1930 \mathrm{~V}$ in January, 2001, and the $\mathrm{DCH}$ has operated at this voltage ever since (i.e. during the acquisition of $\sim 90 \%$ of the data sample through the end of Run 4). At the same time it was decided to add 3500 ppm of water vapour to the gas mixture in order to reduce the probability of electrical discharge.

The BABAR coordinate system is defined by the DCH. Horizontal (x) and vertical (y) axes are specified in terms of the nominal positions of the holes in the DCH endplates into which the sense-wire feed-throughs are inserted. It should be noted that the actual hole positions relative to nominal were surveyed by means of a Coordinate Measuring Machine at the factory of the manufacturer, and that these corrections are incorporated when specifying the end-locations of each individual sense wire. At assembly, care was taken to align these two sets of axes, and to ensure that the line connecting their origins was normal to the surfaces of both plates. This line then specifies the $\mathrm{z}$-axis of the BABAR coordinate system, and its direction is chosen to coincide to a good approximation with the axis of the BABAR magnetic field, with positive $\mathrm{z}$ in the direction of the field. In the collider hall, this is also the approximate direction of the high energy $\left(e^{-}\right)$beam; the orientation of the $\mathrm{DCH}$ is then such that the chosen y direction points upward toward the roof, and the x-direction is in the horizontal plane pointing outward from the centre of the PEP II rings. The origin of the coordinate system along the z-axis is then chosen to be located at $37 \mathrm{~cm}$ in the negative $\mathrm{z}$ direction relative to the centre of the magnet cryostat. This defines the Interaction Point (IP in fig.2.4), and is the nominal point at which the $e^{-}$ and $e^{+}$beams collide. This choice of coordinate system matches the asymmetric detector configuration (see fig.4.7(d)), which compensates for the energy asymmetry between the colliding beams in such a way as to yield approximately symmetric detector acceptance in the centre-of-mass frame. Systematic studies making use of charged particle trajectories in this coordinate system are then used to transform local measurements in the other detector subsystems into this DCH frame (e.g. the GA transformation described in Section 2.2).

Typically a charged particle track traversing the full radial extent of the DCH will have $\sim 36 \mathrm{DCH}$ coordinate measurements associated with its trajectory. If the track originated within the PEP II beampipe, 10 SVT coordinates will also be associated with this track. In order to extract precise momentum and position information from these measurements, they are incorporated into a Kalman filter fit which takes into account variation of the magnetic field with position, multiple scattering and energy loss in the materials of the $B A B A R$ detector traversed, and how these corrections depend on the mass hypothesis being made concerning the identity of the charged particle. The momentum resolution which results from this process can be extracted directly from cosmic ray data. Cosmic rays which enter the DCH preferentialy in the upper half of the chamber, pass close to the collision region within the beampipe, and exit through the bottom half of the DCH, can be reconstructed separately as upper DCH and lower DCH tracks. By comparing the upper and lower DCH versions of the same track, estimates of transverse momentum resolution, 
$\mathrm{dp}_{T} / \mathrm{p}_{T}$, can be obtained from actual DCH and SVT measurements (as opposed to Monte Carlo simulation). The momentum resolution dependence on $\mathrm{p}_{T}$ obtained in this way can be parametrized by [79]:

$$
\frac{\mathrm{dp}_{T}}{\mathrm{p}_{T}}=\left(0.45+0.13 \times \mathrm{p}_{T}\right) \%
$$

with $\mathrm{p}_{T}$ in $\mathrm{GeV} / c$. The first term represents the multiple scattering limit, while the second term results if the precision of the measurement of the sagitta of the curved trajectory in the transverse (i.e. $\mathrm{x}-\mathrm{y}$ ) plane remains constant while the sagitta itself decreases as $1 / \mathrm{p}_{T}$. It follows that the resolution varies from $\sim 0.5 \%$ at $\sim 0.5 \mathrm{GeV} / c$ to $\sim 1 \%$ at $5 \mathrm{GeV} / c$. This is in excellent agreement with the design objective, and reflects the high quality of $B A B A R$ charged track reconstruction. It is this quality which yields the excellent invariant mass and vertex precision discussed in detail in Chapters 3 and 4 of this thesis.

In addition to providing coordinate information through drift-time measurement, the DCH also provides specific ionisation $(\mathrm{dE} / \mathrm{dx})$ information by means of the pulse-height recorded simultaneously for the relevant sense wire. After calibration, this measures the ionization energy loss in the DCH cell in question, and, when normalized to unit path length, provides a local dE/dx sampling. Since such samples follow a Landau distribution, they will not yield a reliable estimate of the most-probable $\mathrm{dE} / \mathrm{dx}$ value if simply averaged; it is the most-probable value which exhibits a Bethe-Bloch dependence on velocity, and hence yields particle identification information. In order to obtain a reliable estimate of the most-probable $\mathrm{dE} / \mathrm{dx}$ value for the track in question, the $20 \%$ of $\mathrm{dE} / \mathrm{dx}$ samples with the largest values are discarded, and the mean value for the remaining samples is calculated. This removes the so-called "Landau tail", and the resulting "80\% Truncated Mean" provides a good estimate of the most-probable $\mathrm{dE} / \mathrm{dx}$ value. For a typical track with $\sim 36$ samples, $\sim 29$ are used to obtain the $\mathrm{dE} / \mathrm{dx}$ estimate. A single sample provides an estimate with $\sim 42 \%$ resolution, and so the resolution in $\mathrm{dE} / \mathrm{dx}$ would be expected to be $\sim 7.5 \%$ of $\mathrm{dE} / \mathrm{dx}$ for a typical track. This is in fact what is observed, and as can be seen in the analyses of Chapters 3 and 4 , the DCH provides very high quality energy-loss information which thus permits excellent mass discrimination for charged particle tracks with lab. momentum below $\sim 1.2 \mathrm{GeV} / c$. This is made particularly clear by figs. 4.10, 4.14, and 4.31. Inspection of the corresponding consistency plots reveals a slight skewing toward high $\mathrm{dE} / \mathrm{dx}$ measurement values; this is the imprint of the underlying Landau distribution, which persists because of the approximate nature of the truncated mean procedure used to estimate the most-probable energy loss value.

In summary, the DCH has delivered the performance anticipated in the BABAR design report in terms of both momentum resolution and particle identification capability. This is discussed in much greater detail in refce. [79]. 


\subsection{Detector of Internally Reflected Cherenkov Radiation (DIRC)}

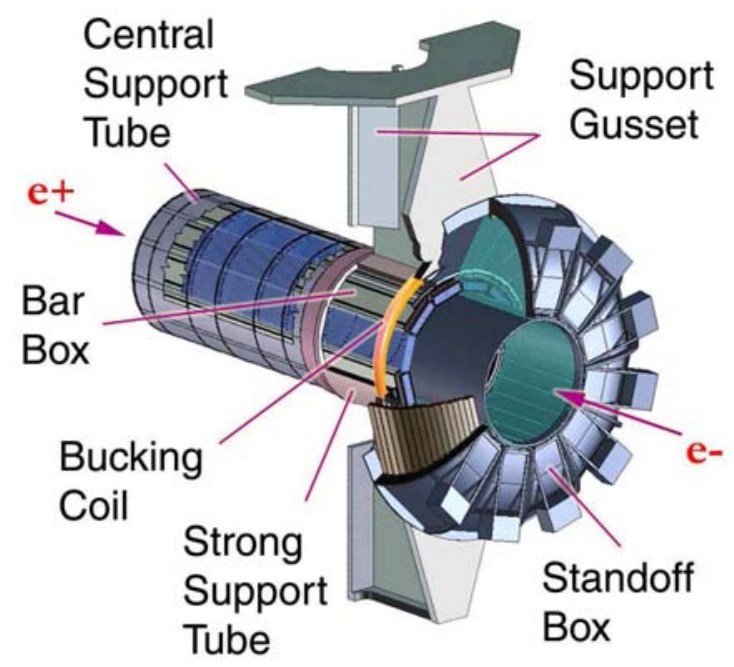

Figure 2.6: Shown is an illustration of the DIRC [79] particle identification system. The DIRC comprises 144 thin quartz bars positioned in a 12-sided cylindrical polygon parallel to the beam-pipe. The cylinder of the DIRC is cantilevered off the standoff box (SOB). Therefore, the SOB acts as a support structure and houses the water used to direct the light from the quartz bars to the photomultiplier tubes.

Distinguishing hadrons from leptons over a large range of solid angle and momentum is an essential requirement for meeting the physics objectives of $B A B A R$. In particular, measurements of $C P$ violation require particle identification (PID), both to reconstruct exclusive final states and to tag the quark content of B decays. Information from the SVT, DCH, EMC, and the IFR is used to identify electrons and muons and contributes to hadron identification. However, an additional dedicated particle identification system is essential to distinguish charged pions from kaons for momenta greater than $\sim 0.6 \mathrm{GeV} / c$, and from protons for momenta above $\sim 1.2 \mathrm{GeV} / \mathrm{c}$ as this is required to obtain efficient tagging and event reconstruction. In order to meet this requirement, a DIRC system is incorporated into the barrel region of the detector. It is azimuthally symmetric w.r.t. to the $z$-axis, and its coverage extends in polar angle from $25.5^{\circ}$ to $141.4^{\circ}$; in azimuth, it covers $\sim 92 \%$ of $2 \pi$, since there are small gaps in coverage between the bar boxes.

The DIRC is an internal reflection imaging device that uses 144 thin quartz ${ }^{1}$ bars arranged in a 12-sided polygon around the beam line as illustrated in fig. 2.6. This maximizes azimuthal coverage, simplifies construction, and minimizes edge effects.

For sufficiently fast charged particles, some part of the Cherenkov radiation cone emitted by the particle $\left(\Theta_{c}(E)=\cos ^{-1}[1 / n \beta]\right.$, with $\left.n \sim 1.473\right)$ is captured by internal reflection in the bar and transmitted to the photon detector array located at the backward end of

\footnotetext{
${ }^{1}$ Actually synthetic fused silica glass is used, not crystalline quartz which is birefringent, but the term "quartz" is used colloquially.
} 
the detector. (Forward-going light is first reflected from a mirror located on the end of the bar.) The high optical quality of the quartz, along with rigorous specifications of squareness, flatness and polish of the bar surfaces, preserves the angle of the emitted Cherenkov light and minimises absorption. The measurement of this angle, in conjunction with knowledge of the track angle and momentum from the drift chamber, allows a determination of the particle mass. An advantage of the DIRC for an asymmetric collider is that the high momentum tracks are boosted forward, which gives a much higher light yield than for particles at normal incidence. This is due to two effects: the longer path length in the quartz and a larger fraction of the produced light being internally reflected in the bar.

Each quartz bar is $1.7 \mathrm{~cm}$ thick, $3.5 \mathrm{~cm}$ wide, and $490 \mathrm{~cm}$ long, and is constructed by glueing four shorter bars end-to-end. The total radial space occupied by the DIRC, including quartz thickness, sagitta from the polygonal shape, mechanical supports, and a $1 \mathrm{~cm}$ clearance on each side, is $10 \mathrm{~cm}$. This material represents $0.19 X_{0}$ (radiation length) at normal incidence. An effort has been made to minimize both the radial thickness and the amount of material, since these increase the size and cost of the barrel calorimeter while degrading its performance.

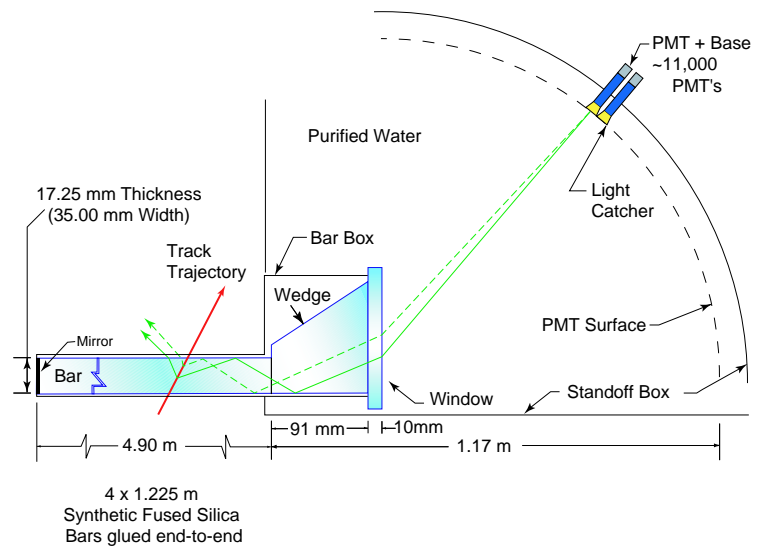

Figure 2.7: Shown is a schematic of a single quartz bar in the DIRC detector illustrating the principle of the detector [79]. As a particle enters the quartz bar, a Cherenkov cone is created which then propagates to the end of the bar and onto the PMT detector surface.

Fig. 2.7 illustrates the principle of the DIRC. A quartz "wedge" is glued to the readout end of each bar. This reflects the lower Cherenkov ring image onto the upper one, moving it inside the PMT acceptance. The wedge is a $9.1 \mathrm{~cm}$ long block of quartz with the same width as the bars, and a trapezoidal profile, $2.7 \mathrm{~cm}$ high at the bar end and $7.9 \mathrm{~cm}$ high where it is glued to the quartz window which provides the interface to the water-filled standoff-box (SOB). Total internal reflection on all sides of the quartz wedge provides nearly loss-free transmission.

Within the water, the Cherenkov cone image is allowed to expand in diameter and is then detected by an array of $\sim 11,000$ conventional $2.5 \mathrm{~cm}$-diameter photomultiplier tubes located on the exterior of the tank. They are organized in a close-packed array at a distance 
of about $120 \mathrm{~cm}$ from the end of the radiator bars. Light-catcher attachments provide increased light-collection efficiency. Water is used because if pure it has good transmission properties for the Cherenkov spectrum detected by the PMTs, has a similar refractive index to that of quartz, a good match in dispersion, and it is also very inexpensive.

The phototubes, together with modular bases, are located in a gas-tight volume as protection against helium leaks from the drift chamber. The photodetection surface approximates a partial cylindrical section in elevation and a toroid when viewed from the end. To maintain good PMT single photon efficiency, the SOB is surrounded by a steel box which, along with a bucking coil, provides adequate magnetic shielding for the phototubes. 


\subsection{ElectroMagnetic Calorimeter (EMC)}

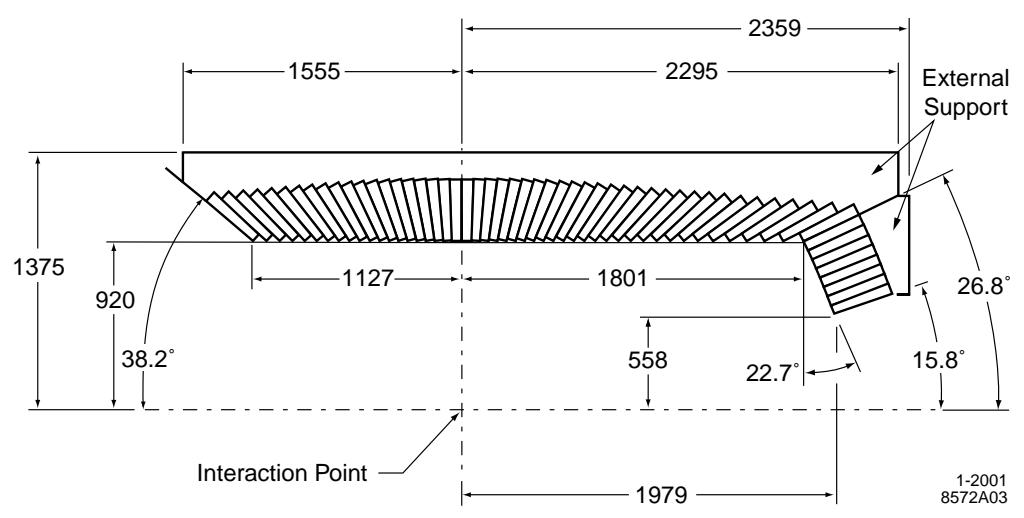

Figure 2.8: A longitudinal cross section of the EMC (only the top half is shown) indicating the arrangement of the 56 crystal rings. The detector is symmetric around the $z$-axis. All dimensions are given in $\mathrm{mm}$.

The Electromagnetic Calorimeter (EMC) is designed to detect electromagnetic showers with excellent energy and angular resolution over the $20 \mathrm{MeV}$ to $4 \mathrm{GeV}$ energy range. This capability allows the detection of photons from secondary hadronic decays and radiative and electromagnetic processes (along with $n$ 's and hadronic showers from $K_{L}^{0}$ 's). By means of electron identification, the EMC contributes to $J / \psi$ and $\tau$ reconstruction, along with flavor identification of neutral $B$-mesons via semileptonic decays.

The EMC consists of a cylindrical barrel (5760 crystals), and a conical forward endcap (820 crystals). A longitudinal cross section of the EMC indicating the arrangement of the 56 crystal rings, is shown in fig. 2.8. The detector is symmetric around the $z$-axis, its coverage extends in polar angle from $15.8^{\circ}$ to $140.8^{\circ}$ (90\% in the C.M. system) and over the full azimuth.

Thallium-doped caesium iodide ( $\mathrm{CsI}(\mathrm{Tl})$ ) scintillating crystals (shown in fig. 2.9), have a short radiation length $(1.85 \mathrm{~cm})$ allowing for shower containment at BABAR energies with a relatively compact design. Their high light yield and emission spectrum permit efficient use of silicon photodiodes which operate well in magnetic fields. These are coupled to the back surface of the crystals with a polystyrene plate.

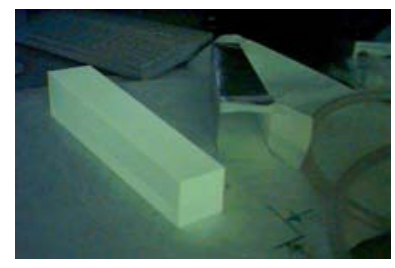

Figure 2.9: A thallium-doped caesium iodide [CsI(Tl)] Crystal.

To enable the calorimeter to make an accurate measurement of the energy of the particles at the time of creation, and due to the sensitivity of the $\pi^{0}$ efficiency to the 
minimum detectable photon energy, it is important to keep the amount of material in front of the EMC to the lowest possible level, so as to reduce the chance of preshower and $\gamma$ conversions. The barrel and the outer five rings of the endcap have $\sim 0.3-0.6$ $X_{0}$ of material in front of the crystal faces. The three innermost rings of the endcap are shadowed by the SVT support structure (although the main crystal support structure is at the rear of the crystal to minimise material) and electronics as well as the B1 bending dipole, resulting in up to $3 X_{0}$ for the innermost ring. The principal purpose of the two innermost rings is to enhance shower containment for particles close to the acceptance limit. 


\subsection{Instrumented Flux Return (IFR)}

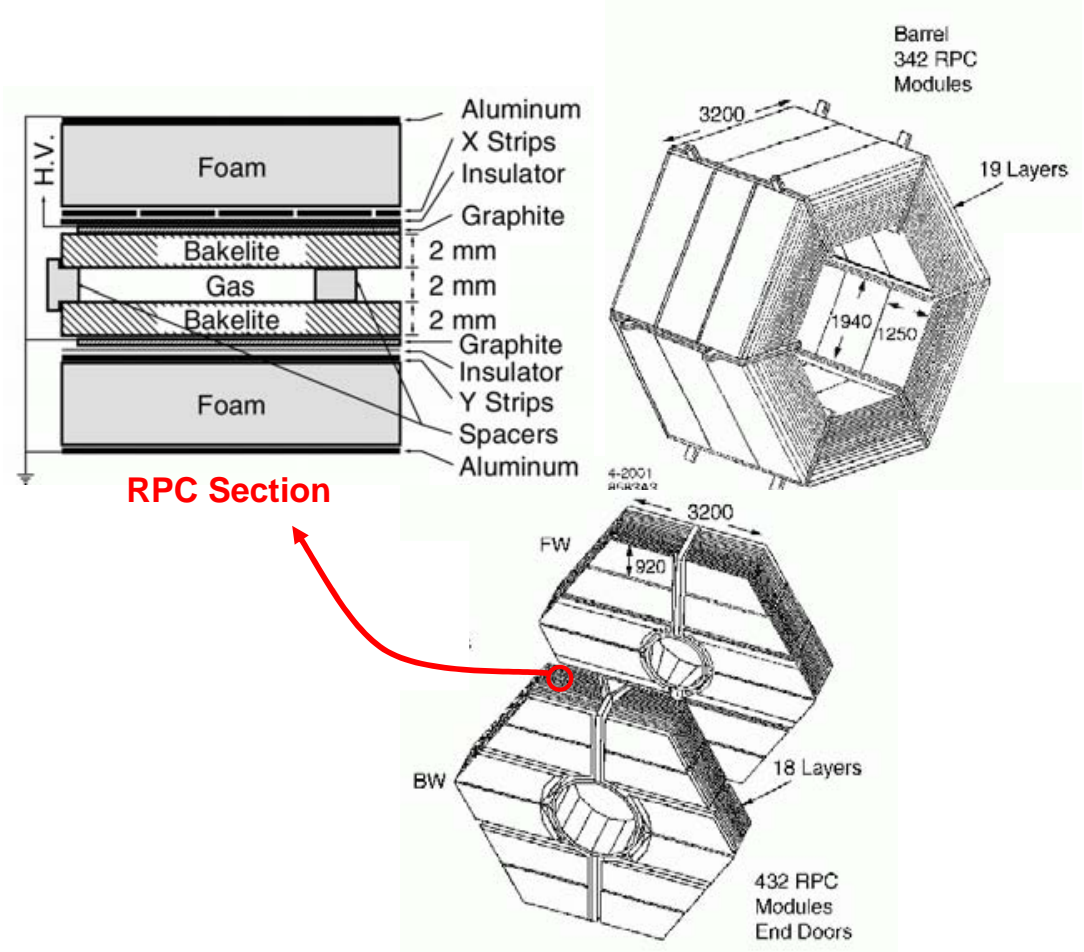

Figure 2.10: Diagram showing the Instrumented Flux Return (IFR) [79]. Resistive Plate Chambers are inserted between the many iron plates that serve as the magnetic flux return to detect muons and showers from neutral hadrons.

Surrounding the EMC is a large iron yoke that is instrumented to provide muon and hadron identification. The iron structure also provides the external flux return for the magnetic field from the superconducting solenoid. The primary purpose of the IFR is identify charged particles as muons; this information can then be used in the same way as that for electrons identified with the EMC. The IFR can also be used in $K_{L}^{0}$ reconstruction to detect neutral energy appearance, with the corresponding position in the IFR then providing a direction vector to be compared to the missing momentum vector in an exclusive final state. The IFR can also be used as a tail catcher for electromagnetic or hadronic showers generated in the EMC.

The IFR, illustrated in fig. 2.10 consists of 18 layers of steel, of increasing thickness from $2 \mathrm{~cm}$ at the inner layer to $10 \mathrm{~cm}$ at the outer layer, which screen out pions by preventing "punch through". Single gap resistive plate chambers (RPC's) are inserted between the steel absorber plates, and these detect streamers from ionizing particles via external capacitive readout strips. There are 19 layers of RPC's in the barrel section and 18 in the end doors.

Unfortunately, the IFR performance has degraded over time, and has been predicted to continue to do so. The Forward end door RPCs were replaced in the summer of 2002, and the barrel RPCs are being replaced with Limited Streamer Tube technology before 
efficiency reaches unacceptable levels. 


\subsection{Trigger}

Data retrieved from the subsystems are collected by the "front-end" electronics. Selected data from the DCH, EMC and IFR are then used to form Level 1 (L1) trigger "primitives"; these are the raw data such as track $p_{T}$ from the $\mathrm{DCH}$, energy deposited in the EMC and clusters in the IFR.

The DCH track primitives are formed from track segments by the TSF (Track Segment Finding) modules. These modules also classify the quality of track segments. From the TSF modules, the data are sent to the BLT (Binary Link Tracker), which forms complete tracks from the segments which are required to either reach the outer layer of the DCH and be present in either at least eight of the $10 \mathrm{DCH}$ superlayers, or penetrate to the middle layer of the DCH. EMC primitives are formed using data collected from the 280 towers from the barrel and the endcap calorimeter (which are divided into, $7 \times 40$ and $8 \times 3$ regions of $\theta$ and $\phi$ respectively). The energy is then summed over $\theta$ for each $\phi$ region and compared to various thresholds. The IFR primitives are used to identify di-muon and cosmic ray events. These are defined by $O R$ signals of all $\phi$ read-out strips in eight layers in each sector, where a sector is one of ten regions of the IFR (six barrel sextants, four half end doors). A trigger object is formed when four out of the eight trigger layers have hits within a time window of 134 ns.

These data are then passed through the Global Trigger, where a decision is made to accept or reject the event based on predetermined logic and prescaling rates (the logic and rates are not fixed and are highly configurable). A L1 signal is sent through the Read Out Modules (ROMs) back to the "front-end" where the data are finally extracted. These data are then shipped to the event builder ROMs which assemble the event so that it can be processed by Level 3 (L3) trigger farm nodes and other "Online Event Processing" nodes assigned to monitoring tasks.

The L3 trigger, a software implementation, further classifies data coming from Level 1. An attempt is made to reject background, based on event topology for example, and classify certain events, such as two prongs, which are useful in detector calibration. The $\mathrm{L} 1$ rate is $>2 \mathrm{kHz}$ and has the capability to handle $>4 \mathrm{kHz}$ without significant deadtime. The L3 rate is about $15 \mathrm{nb}$ (i.e. $150 \mathrm{~Hz}$ at $10^{34}$ ). The high "physics" rate Bhabhas are heavily prescaled to reduce this rate.

Events which pass the L3 Trigger are sent to the logging manager which writes the data to an eXtended Tagged Container (XTC). Each XTC file represents a single "run", typically 1 hour of continuous data acquisition time. At the end of a run, the XTC file is archived and made available for further processing.

The data are then sent to the Prompt Calibration farm, and then to Event Reconstruction at which time the events are sequentially calibrated and reconstructed using custom designed algorithms, and written into the event store.

An intermediate Level 2 trigger has not been implemented in BABAR, even with in- 
creased data rates and higher luminosity, because the L3 capacity is easily able to handle the observed rates. 


\section{Chapter 3}

\section{Search for $\Theta_{5}(1540)^{+}$Production in $e^{+} e^{-}$Interactions}

\subsection{Introduction}

This chapter describes the inclusive search for the $\Theta_{5}^{+}$member of the anti-decuplet and octet in the $e^{+} e^{-} \rightarrow$ hadrons, data sample. The production of a wide range of hadrons in $e^{+} e^{-}$annihilations seems to occur democratically. The abundant production of ordinary baryons in $e^{+} e^{-} \rightarrow$ hadrons was a surprise in the early days of PETRA and PEP. Since then, more and more baryon states, as well as 'surprising' scalar and tensor meson states have been observed; their production rates are summarized in fig. 3.1. BABAR has the ability to do much better on all points at $\sqrt{s} \sim 10 \mathrm{GeV}$.

The key feature is that baryons, vector and tensor mesons all have a production rate given approximately by $e^{-M / T}$, where $T \sim 103 \mathrm{MeV}$ and $M$ is the particle mass. The dominant parameter controlling the rate seems to be the mass, despite the very different quark content. The ordinary baryon production rates provide a useful reference in considering models for pentaquark production: one might expect rates as high as the lower baryon line, or about $8 \times 10^{-4} \Theta_{5}^{+}$per continuum hadronic event; upper limits at this level or an order of magnitude lower are interesting to hadronization modelers and spectroscopists alike. Predictions for production in $B$ decays are also available, therefore it is also important to provide limits for testing these.

\subsection{Data Set}

The integrated luminosity used in this analysis is the combination of on-resonance data taken at $\sqrt{s}=10.58 \mathrm{GeV}$, primarily for the study of $B$-mesons, corresponding to 111.59 $\mathrm{fb}^{-1}$, along with off-resonance data taken at a C.M. energy of $0.040 \mathrm{GeV}$ lower, the primary reason for which is for $B$-meson background studies, this corresponds to 11.96 $\mathrm{fb}^{-1}$. The data were obtained in the period period October 1999 to June 2003.

No explicit hadronic event selection is performed, in order to make this analysis as 


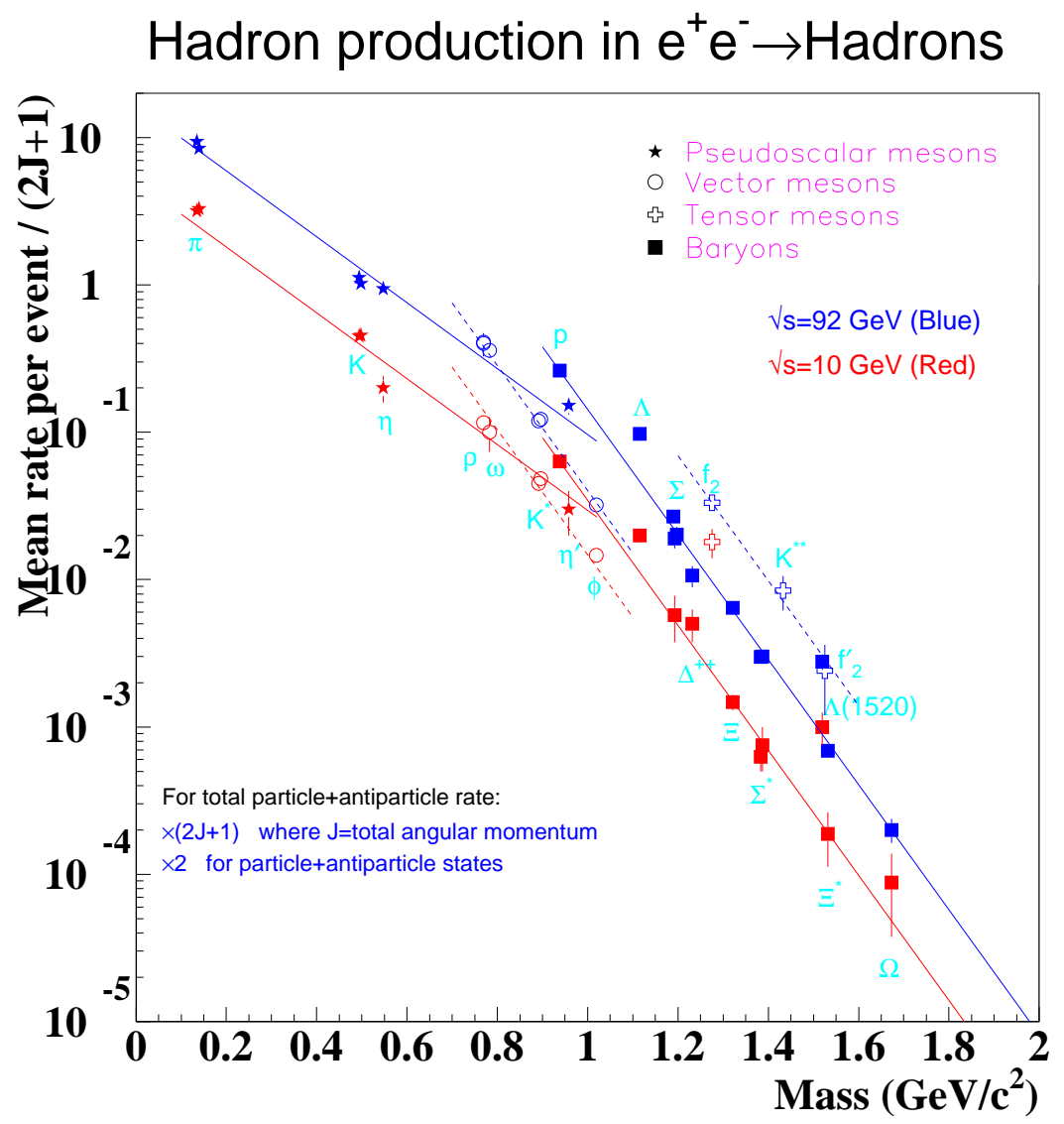

Figure 3.1: Compilation of light (no $c$ or $b$ quarks) hadron production rates in $e^{+} e^{-} \rightarrow$ hadrons from the PDG. $J$ is the particle spin, and rates correspond to particle states only; for particles with distinct antiparticle states, the total particle and anti-particle production rate is twice that shown.

inclusive as possible and maintain maximum signal efficiency. The vast majority of candidates are in hadronic events; lepton-pairs are expected to contribute negligible background in the signal regions; two-photon and ISR events contribute only at low momenta. The acceptance for known processes is believed to be well understood, the integrated luminosity $\left(\sim 123 \mathrm{fb}^{-1}\right)$ and known cross sections can be used to evaluate production rates and set limits in the continuum and $\Upsilon(4 S)$ decays. If a signal were found, an attempt to isolate the source by imposing selection criteria on the event properties, would be possible. 


\subsection{Event Preselection}

\subsubsection{Reconstruction and Selection of Inclusive $K_{S}^{0}$ Candidates}

$K_{S}^{0}$ mesons are selected by attempting to isolate candidates that originate from the interaction region (IR). The method and procedures are outlined below. Initially opposite sign combinations of tracks with no kinematic constraints and the pion mass hypothesis assigned, are vertexed. A mass window of $\pm 50 \mathrm{MeV} / c^{2}$ around the PDG value for the $K_{S}^{0}$ mass is used to select candidates. The distance between the point of closest approach (POCA) of the daughter tracks is required to be less than $6 \mathrm{~mm}$. This retains all visible $K_{S}^{0}$ candidates in the mass spectrum with a substantial reduction in background.

In this context, the IR is reconstructed as a straight line through the collision region ("Collision Axis"). This is achieved by using an averaged run-by-run coordinate position in the center of the IR, referred to as the Interaction Point (IP). The IP has an associated covariance matrix describing the ellipsoidal shape of the IR within the BABAR coordinate system. A direction ${ }^{1}$ can be derived from this matrix and a straight line following this through the IP is used to define the Collision Axis.

The reconstructed $K_{S}^{0}$ trajectory (the momentum vector through the $\pi^{+} \pi^{-}$vertex) should extrapolate close to the Collision Axis for real $K_{S}^{0}$ candidates for the majority of physics processes that are of interest. Therefore, to ensure the required consistency with an origin in the IR, selection criteria are applied to the distance of closest approach (DOCA) of the $K_{S}^{0}$ trajectory to the Collision Axis. Initially this is determined in the transverse plane, by calculating $R_{x y}$ - the radial separation distance.

$$
R_{x y}=\sqrt{\left[X_{K_{S}^{0}}^{a p}-X_{\text {Beam }}\left(Z_{K_{S}^{0}}^{a p}\right]^{2}+\left[Y_{K_{S}^{0}}^{a p}-Y_{\text {Beam }}\left(Z_{K_{S}^{0}}^{a p}\right)\right]^{2}\right.}
$$

where $\left(X_{K_{S}^{0}}^{a p}, Y_{K_{S}^{0}}^{a p}, Z_{K_{S}^{0}}^{a p}\right)$ is the point of closest approach between the fitted momentum vector of the $K_{S}^{0}$ candidate and the collision axis, and:

$$
\begin{aligned}
& X_{\text {Beam }}\left(Z_{K_{S}^{0}}^{a p}\right)=X_{I P}+\left(\frac{d x}{d z}\right) \times\left(Z_{K_{S}^{0}}^{a p}-Z_{I P}\right) \\
& Y_{\text {Beam }}\left(Z_{K_{S}^{0}}^{a p}\right)=Y_{I P}+\left(\frac{d y}{d z}\right) \times\left(Z_{K_{S}^{0}}^{a p}-Z_{I P}\right)
\end{aligned}
$$

Here, $\frac{d y}{d z}$ and $\frac{d x}{d z}$ define the slope of the Collision Axis. Further to this, a requirement is placed on the separation between $Z_{K_{S}^{0}}^{a p}$ and the $z$-coordinate of the IP $\left(Z_{I P}\right)$.

The spread in $z,\left|Z^{a p}-Z_{I P}\right|$, is determined by taking into account the known measurements of the Beam Spot $^{2}$ and degradation in resolution by reconstruction effects. The

\footnotetext{
${ }^{1}$ See http://www.slac.stanford.edu/ wmd/bbkinematics/Collision_Axis_Tilt.note

${ }^{2}$ The size of the luminous region is obtained from vertex reconstruction: $\sigma_{x} \sim 110-120 \mu \mathrm{m}$

$\sigma_{y} \sim 25-35 \mu \mathrm{m}$

$\sigma_{z} \sim 7.5-8.0 \mathrm{~mm}$ : in the early days, this was $8.3 \mathrm{~mm}$, but it has decreased with time because of additional r.f., which yields a smaller bunch length
} 


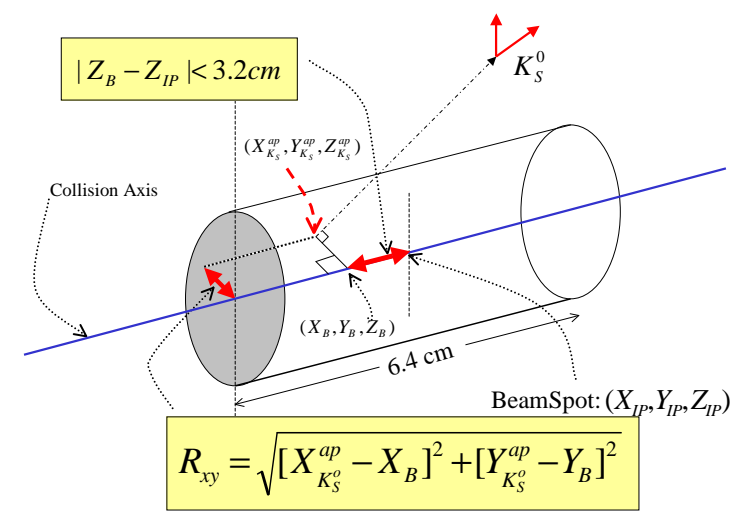

Figure 3.2: A sketch illustrating the dynamics of a $K_{S}^{0}$ decay. The Interaction Region is treated as cylindrical in nature, and the $K_{S}^{0}$ trajectory is required to be consistent with originating from this region.

treatment of the IR and the selection criteria that are used to define it are illustrated in fig. 3.2. The requirements on $R_{x y}$ and $\left|Z^{a p}-Z_{I P}\right|$ are $0.5 \mathrm{~cm}$, and $3.2 \mathrm{~cm}$ respectively. In effect this is a cylindrical treatment of the ellipsoid region, treating it in terms of simple geometry.

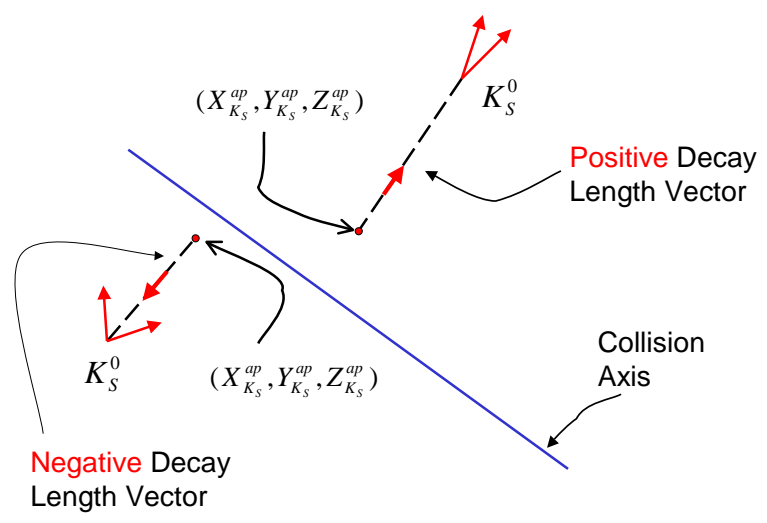

Figure 3.3: A sketch depicting reconstructed $K_{S}^{0}$ candidate vertices with respect to the collision axis. Vertices which are inverted with respect to this have a negative decay length vector and when calculated also display a negative Signed Flight Length.

When reconstructing $K_{S}^{0}$ candidates, it is possible to reconstruct vertices that are "behind" or "inverted" with respect to the IP, that is, the $K_{S}^{0}$ trajectory points from the vertex toward the Collision Axis rather than away from it, as illustrated in fig. 3.3, showing typical topologies for vertices that are both rejected and selected with this criterion. To remove "inverted" candidates the Signed Flight Length (SFL) is defined with respect to the Collision Axis as,

$$
S F L=(\hat{n} \cdot \hat{p}) \times \sqrt{\left(x_{v}-x_{K_{S}^{0}}^{0}\right)^{2}+\left(y_{v}-y_{K_{S}^{0}}^{0}\right)^{2}+\left(z_{v}-z_{K_{S}^{0}}^{0}\right)^{2}},
$$


where $\hat{n}$ is a unit vector in the direction from the IP to the $K_{S}^{0}$ decay vertex, $\left(x_{v}, y_{v}, z_{v}\right)$ and $\hat{p}$ is a unit vector along the momentum of the fitted $K_{S}^{0}$ candidate.

The distribution of $S F L$ for combinatoric pairs (coincidentally giving the $K_{S}^{0}$ mass) from the IP is peaked at and symmetric about zero, with a core width of $\sim 1 \mathrm{~mm}$ and tails extending several $\mathrm{mm}$ in both positive and negative directions, whereas the distribution for true $K_{S}^{0}$ is entirely positive. Requiring $S F L>0$ reduces the background by nearly a factor of two with negligible signal loss; harder cuts can yield a very clean sample, but with loss in efficiency.

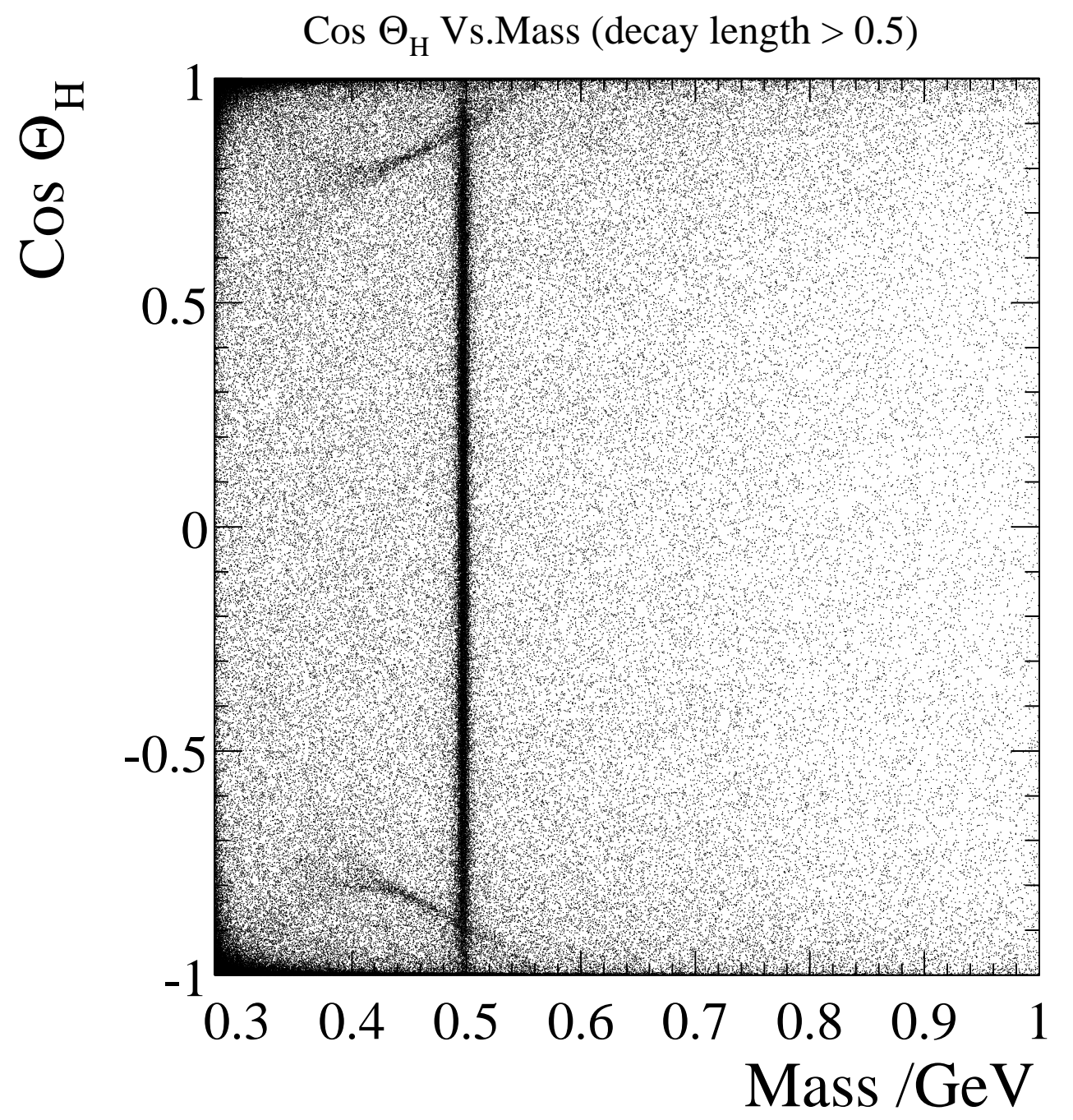

Figure 3.4: Mass vs. $\cos \left(\Theta_{H}\right)$. A $\Lambda^{0}$ presence is easily recognized at $\sim \pm 0.8$ and $\gamma \rightarrow e^{+} e^{-}$ at $\sim \pm 1.0$ in the $\cos \left(\Theta_{H}\right)$ plane and the $K_{S}^{0}$ at $\sim 0.497 \mathrm{GeVc}^{-2}$

The distribution in the cosine of the helicity angle $\left(\Theta_{H}\right)$ of the $\pi^{+}$from the candidate $K_{S}^{0}$ is then considered; $\Theta_{H}$ is the angle between the $\pi^{+}$direction in the $K_{S}^{0}$ rest frame and the $K_{S}^{0}$ direction in the lab. frame. The distribution of $\cos \Theta_{H}$ is flat for true $K_{S}^{0}$ meson decays. This distribution is plotted against $\pi^{+} \pi^{-}$mass in fig. 3.4; the peaks near $\cos \Theta_{H}= \pm 1$ result from $\gamma$ conversions (when the electrons are assigned the pion 
mass) ${ }^{3}$, and the $\Lambda$ (where the $p$ or $\bar{p}$ has been assigned pion mass) populates the regions $\pm(0.8 \rightarrow 0.9)$. Therefore candidates are rejected where $\left|\cos \Theta_{H}\right|>0.8$, as this eliminates clearly defined backgrounds in the $K_{S}^{0}$ mass window.

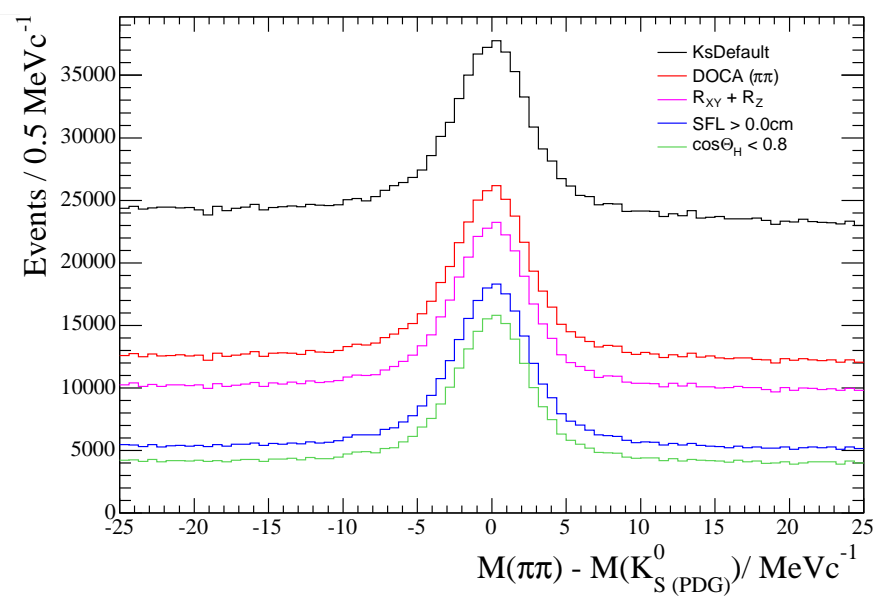

Figure 3.5: $\pi^{+} \pi^{-}$invariant mass distributions for $K_{S}^{0}$ candidates in the data from all pairs of oppositely charged tracks, assigned the charged pion mass and with no kinematic constraints (upper histogram), and after the cuts on $\operatorname{DOCA}\left(\pi^{+}, \pi^{-}\right)$(red histogram), $R_{K_{S}^{0}}^{x y}$ and $R_{K_{S}^{0}}^{z}$ (magenta), $S F L>0$ (blue), and $\left|\cos \Theta_{H}\right|$ (lower, green histogram) given in the text.

The distribution of $\pi^{+} \pi^{-}$invariant mass is shown in fig. 3.5, illustrating the effect of the selection criteria so far on the reconstructed distribution. There is significant background suppression from these criteria (factor of $\sim 6$ ) with negligible reduction in signal. The remaining background is generally combinatorial. No particle identification requirements were imposed at this stage.

The selection employed so far is as follows:

$\begin{array}{lll}\operatorname{DOCA}\left(\pi^{+}, \pi^{-}\right) & <6 & \mathrm{~mm} \\ \mathrm{R}_{x y}^{K_{S}^{0}} & <6 & \mathrm{~mm} \\ \mathrm{R}_{z}^{K_{S}^{0}} & <3.2 \mathrm{~mm} \\ \mathrm{SFL} & >0 & \mathrm{~mm} \\ \left|\cos \Theta_{H}\right| & <0.8 & \end{array}$

\subsubsection{Individual Track Candidate Selection}

Initially, all track candidates are required to have $\mathrm{P}_{T}>100 \mathrm{MeV}$ and $\mathrm{P}<10 \mathrm{GeV} / c$. The minimum number of DCH hits allowed is 10, thereby allowing a measurement of ionization loss $(d E / d x)$. The trajectory of the track is required to be consistent with originating from the IR, as was done similarly for the $K_{S}^{0}$ candidate trajectory (using a helix approximation

\footnotetext{
${ }^{3}$ For an explanation of the mapping of a wrongly assigned particle hypothesis in the $\left[\right.$ mass, $\left.\cos \Theta_{H}\right]$ plane see:

http://www.slac.stanford.edu/ wmd/bbkinematics/mass_helicity_mapping.note
} 
as opposed to the straight line trajectory of the $\left.K_{S}^{0}\right)$. Figure 3.6, shows the distribution of the POCA of the track to the Collision Axis. The red lines correspond to the advertised
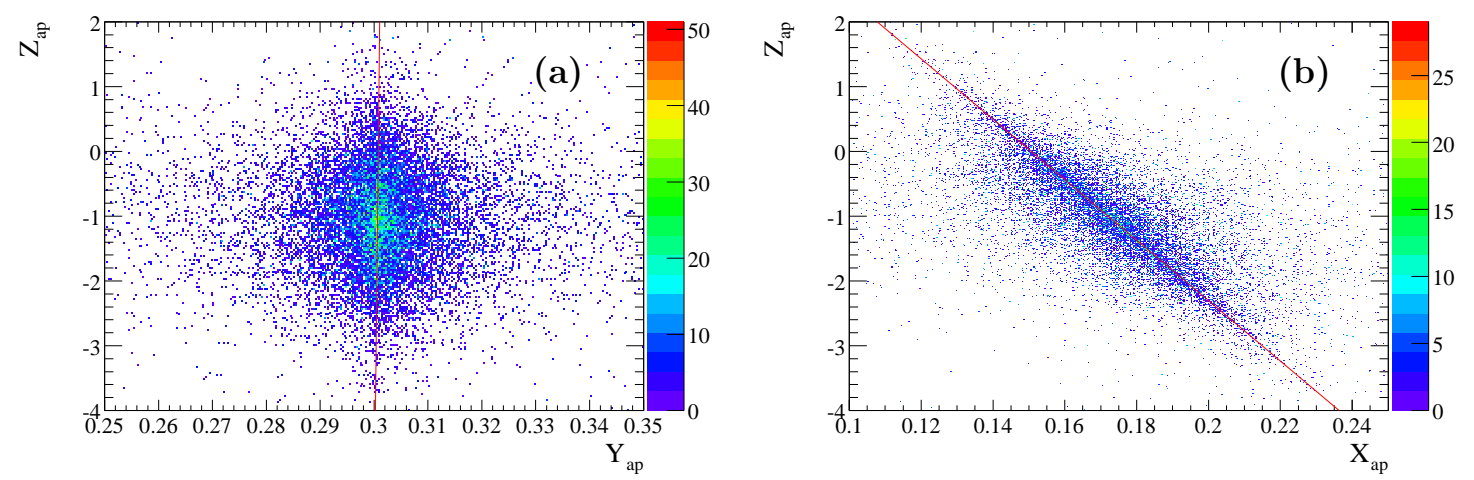

Figure 3.6: (a) The $Y$ vs. $Z$, POCA distribution of Helices to the Collision Axis for a single run, and (b) the $X$ vs. $Z$ POCA distribution. The red line shows the advertised slope of the collision axis for run 14023.

Collision Axis parameters. Similar requirements are applied to the candidate tracks as in the $K_{S}^{0}$ selection, namely:

$$
\begin{array}{ll}
\left|Z_{i p}-Z_{\text {approach }}\right| & \leq 3.2 \mathrm{~cm} \\
R_{x y} & \leq 0.5 \mathrm{~cm} .
\end{array}
$$

\subsection{Proton Identification}

Candidate tracks by definition have a measurement of $d E / d x$ from the DCH associated with them, and tracks that reach the DIRC also have an associated $\Theta_{C}$ measurement from the DIRC. For each track, where appropriate, the DCH and DIRC consistency with the proton hypothesis are calculated and applied. The "proton consistency", $C^{D C H}(p)$ of the DCH with specific energy loss measurements, is defined by,

$$
C^{D C H}(p)=\frac{d E / d x^{\text {measured }}-d E / d x^{\text {calc }}(p)}{d E / d x^{\text {calc }}(p)},
$$

where $d E / d x^{\text {measured }}$ is the energy loss measured in the DCH. $d E / d x^{\text {calc }}(p)$ is calculated using the BABAR Bethe Bloch parametrization for a particle of type $p$ with the momentum measured at the entrance to the DCH. True protons form a band around $C^{D C H}(p)=0$, whereas all other particles form a much denser band that rises with a steep gradient at low momenta, and merges at $\sim 0.8 \mathrm{GeV} / c$.

For tracks which traverse the DIRC, consistency with the calculated emitted Cherenkov angle for a particle with the proton hypothesis is required. This is defined by,

$$
C^{D R C}(p)=\theta_{c}^{\text {measured }}-\theta_{c}(p)
$$



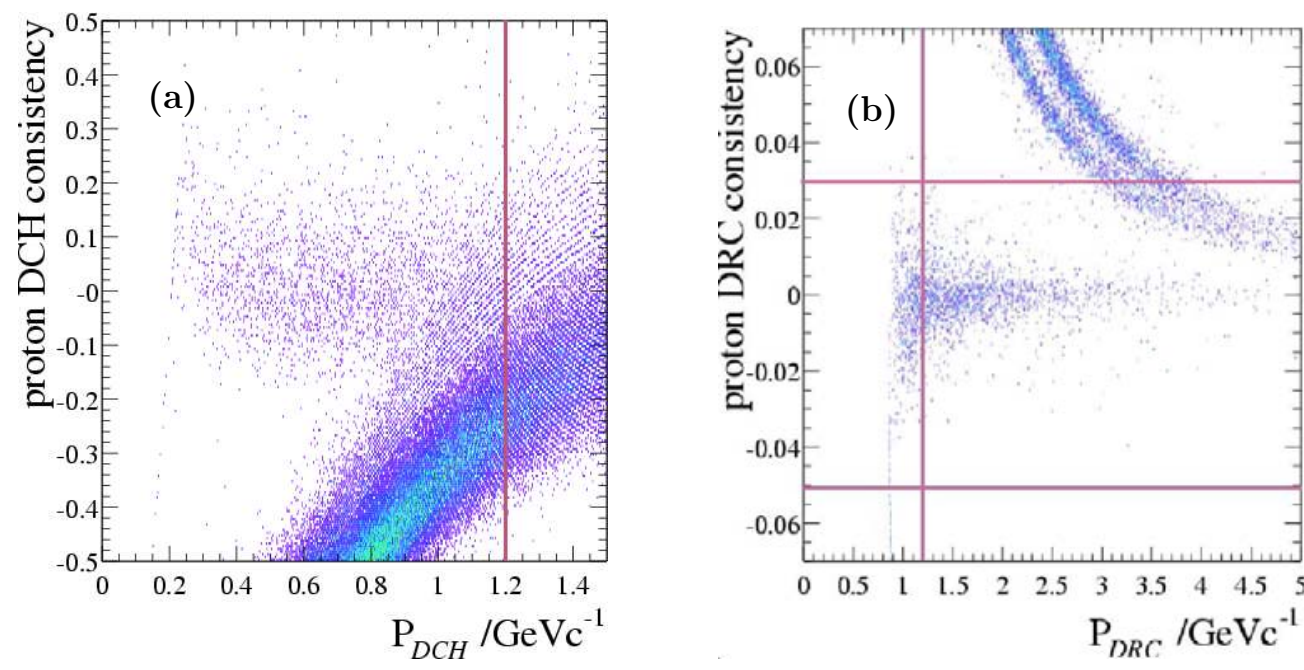

Figure 3.7: (a) DCH consistency vs. momentum $\mathrm{P}$ in the low $\mathrm{P}$ region ; (b) DRC consistency vs. $\mathrm{P}$.

where $\theta_{c}^{\text {measured }}$ is the Cherenkov angle measured in the DIRC, and $\theta_{c}^{p}$ is the expected Cherenkov angle defined by,

$$
\theta_{c}^{p}=\cos ^{-1}\left(1 /\left(\beta_{p} \times 1.473\right)\right)
$$

Here, $\beta_{p}$ is the particle velocity calculated from the momentum at the DIRC and the mass of the proton, and 1.473 is the index of refraction of the DIRC quartz bars.

Initially, a loose acceptance is defined by criteria such as $\left|C^{D C H}(p)\right|<0.5$ for $\mathrm{P}<$ $1.2 \mathrm{GeV} / c$ and $\left|C^{D R C}(p)\right|<0.05$ for $\mathrm{P}>1.2 \mathrm{GeV} / c$ keeping essentially all true particles of type $p$, while reducing background substantially. For example, fig 3.7, shows scatter plots of consistency for the proton hypothesis in the DCH and DIRC vs. momentum. Protons which are above the Cherenkov threshold $(0.8 \mathrm{GeV} / c)$ also form a band around $C^{D R C}(p)=0$, although the band is very wide near threshold due to the small number of photons detected on average; the other particles form bands that descend rapidly at low momenta to converge on zero at higher momenta, and the DIRC efficiency for finding rings is reduced below about $1.5 \mathrm{GeV} / c$. In the intermediate region, $0.8 \rightarrow 1.2 \mathrm{GeV} / c$, the ring finding efficiency is degraded due to being nearer to the detection threshold and therefore producing fewer photons, and $\mathrm{dE} / \mathrm{dx}$ is also less helpful as a significant fraction of the pions are accepted. However the above requirements are still efficient.

It is clear that quite loose cuts on $C^{D C H}(p)$ and $C^{D R C}(p)$ are extremely useful for tracks with momenta below $0.8 \mathrm{GeV} / c$ and above $1.2 \mathrm{GeV} / c$, respectively, although, there is contamination from other charged species; an explanation of how this is handled can be found later in this chapter. 


\subsection{Search for $\Theta_{5}^{+}(1540) \rightarrow p K_{S}^{0}$}

The search for the $\Theta^{+}(1540)$ decaying to a $K_{S}^{0}$ and a proton is described in the following section. After applying the previously described selection criteria, $K_{S}^{0}$ and proton candidates are combined ${ }^{4}$ and the invariant mass distribution of these pairs is examined. In order to get a clean sample, additional requirements were also made as described below.

\subsubsection{Event Selection and Analysis}

The $\pi^{+} \pi^{-}$mass distribution is shown in fig. 3.8(a). The signal is prominent, but the background is not negligible. At this point a harder cut on $S F L$ is considered, however the efficiency at low c.m. momentum $p^{*}$ (the total momentum of the $p K_{S}^{0}$ pair boosted back into the c.m. frame) degrades rapidly when the $S F L$ value is increased. As a systematic check, the analysis was repeated requiring $S F L>0.5 \mathrm{~cm}$, for which the mass distribution is shown in fig. 3.8(b). This sample is much cleaner; but there is a substantial loss of signal at higher $p^{*}$ values. $\mathrm{A} \pm 10 \mathrm{MeV} / c^{2}$ window around the PDG value of the $K_{S}^{0}$ mass is assigned; this allows for the definition of sidebands in the original $\pm 50 \mathrm{MeV} / \mathrm{c}^{2}$ window, although the analysis is such that these were never employed.
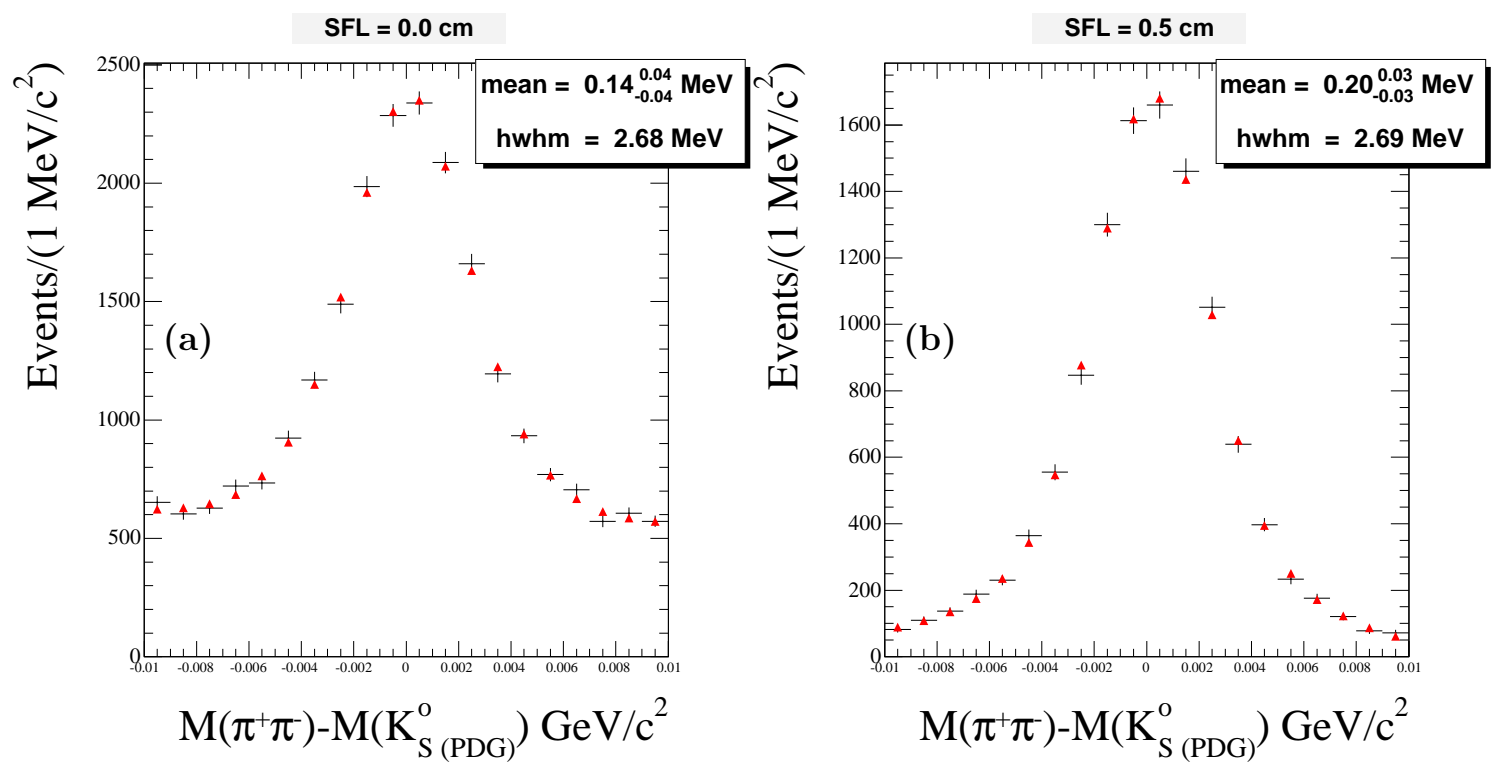

Figure 3.8: Example distributions of the $\pi^{+} \pi^{-}$invariant mass for $K_{S}^{0}$ candidates satisfying the criteria noted in the text; (a) shows candidates with $S F L>0.0 \mathrm{~cm}$; (b) shows the same candidates with $S F L>0.5 \mathrm{~cm}$. The black marker shows the data and the red marker represents the fit at that point.

A further step is taken at this stage to remove candidates which are not consistent with the pion hypothesis. Figure 3.9 shows the DCH pion consistency $C^{D C H}(\pi)$ for the tracks from those $K_{S}^{0}$ candidates passing all other criteria, where $C^{D C H}(\pi)$ is defined to be the same as eqn. 3.5 after substituting the pion hypothesis for the proton hypothesis. It

\footnotetext{
${ }^{4}$ Charge conjugation is implied throughout, unless explicitly stated
} 
is evident that there is an easily identified contamination from particles whose measured value of $d E / d x$ is inconsistent with the pion hypothesis.

Each $K_{S}^{0}$ candidate daughter track is therefore required to satisfy $-0.4<C^{D C H}(\pi)<$ 0.5. The effect of the Landau energy loss distribution is taken into account at low values of momentum, where there is no significant overlap with other charged species, by only selecting pions where $C^{D C H}(\pi)<(3.5-7.5 \times p)$ below $0.5 \mathrm{GeV}$. These criteria are shown by red lines on fig. 3.9 .

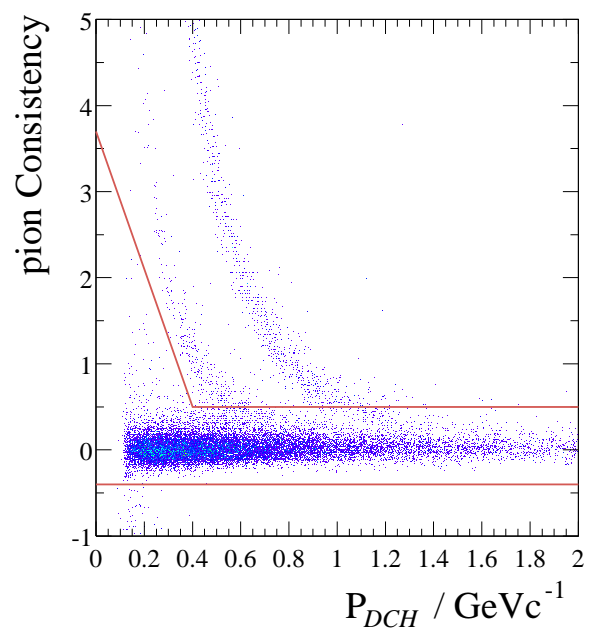

Figure 3.9: The DCH consistency for daughter tracks of the $K_{S}^{0}$ candidates passing the selection criteria outlined previously and within a $\pm 10 \mathrm{MeV} / c^{2}$ window around the $K_{S}^{0}$. Tracks outside the region between the red lines are rejected.

The proton identification is strongly momentum dependent and designed to retain as much readily identifiable signal as possible while reducing the background significantly. $\mathrm{DCH}$ proton consistency is required at all momenta, but tighter criteria are applied in the region where the pion $d E / d x$ band overlaps the proton band; a simple steps and stairs approach is applied to remove the overlap region, the values applied below $1.2 \mathrm{GeV} / c$ are shown in table 3.1. Here $\mathrm{P}_{D C H}$ is the track momentum calculated at the entrance

\begin{tabular}{l|l}
$C^{D C H}(p) \geq$ & $\mathrm{GeV} / c$ \\
\hline-0.20 & $0.25<\mathrm{P}_{D C H} \leq 0.80$ \\
-0.15 & $0.80<\mathrm{P}_{D C H} \leq 0.90$ \\
-0.10 & $0.90<\mathrm{P}_{D C H} \leq 1.00$ \\
-0.00 & $1.00<\mathrm{P}_{D C H} \leq 1.20$ \\
-0.20 & $1.20<\mathrm{P}_{D C H}$
\end{tabular}

Table 3.1: Selection criteria applied to $C^{D C H}(p)$, where $\mathrm{P}_{D C H}$ is the momentum at the entrance to the DCH.

to the DCH. After removal of the overlap region, the sample in this momentum range is essentially pure.

Figure 3.10, shows scatter plots of $C^{D C H}(p)$ vs. $\mathrm{P}_{D C H}$ for the tracks passing these 
criteria. The selection is very loose, and the efficiency very close to unity, for $\mathrm{P}_{D C H} \leq 0.9$ $\mathrm{GeV} / c$; it declines steadily to a minimum of $\sim 55 \%$ for $\mathrm{P}_{D C H} \sim 1.1 \mathrm{GeV} / c$ (this is slightly higher than $50 \%$ due to the asymmetric nature of the Landau Energy Loss distribution); then it rises again and levels off at about $90 \%$ for $\mathrm{P}_{D C H} \geq 1.5 \mathrm{GeV} / c$.

It can be seen from fig. 3.10(b) that for $\mathrm{P}_{D C H}>1.2 \mathrm{GeV} / c$ the sample is contaminated with misidentified pions. Therefore, in addition to the DIRC consistency the track is also required to be a identified as a proton according to the criteria of "pLHTight", one of the $B A B A R$ standard particle ID algorithms. This algorithm takes all the DIRC information (measured and expected number of photons, momentum, Cherenkov angle etc.) as well as $d E / d x$ into account and is quite efficient in this region.

Further to this, a DOCA of $<0.6 \mathrm{~cm}$ is required between the $K_{S}^{0}$ flight path and the proton trajectory.
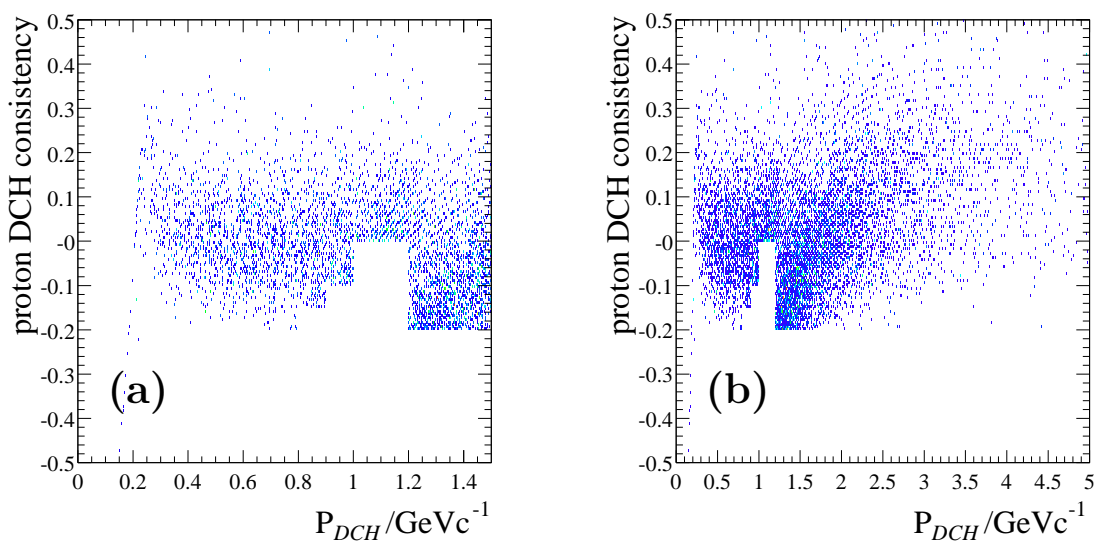

Figure 3.10: DCH consistency vs. momentum at the entrance to the DCH in (a) the low and (b) total momentum regions.

The distribution of the $p K_{S}^{0}$ invariant mass for the selected pairs is shown in fig. 3.11. There is a clear peak at $\sim 2.3 \mathrm{GeV} / c^{2}$ from $\Lambda_{c}^{+} \rightarrow p K_{S}^{0}$ but no other obvious structure. The $\Lambda_{c}^{+}$peak in fig. 3.11(b) demonstrates a mass resolution of better than $12 \mathrm{MeV} / c^{2}$ for a particle at this mass and $Q$ value (i.e. the value, given by $\left.\left(m\left(K_{S}^{0} p\right)-m_{\text {thresh }}\right)\right)$, and the detectors sensitivity to the presence of a very narrow resonance. Figure 3.11(c) focusses in on the range $1.4-1.6 \mathrm{GeV} / c^{2}$; the $\Theta_{5}^{+}$has been reported in the vicinity of $1.54 \mathrm{GeV} / c^{2}$. If the mass splitting between levels of the decuplet is equal, as predicted by Diakonov et al. [25], then the $\Sigma_{5}^{+}$, decaying into $p K_{S}^{0}$, would be expected in the $1.6-1.8 \mathrm{GeV} / c^{2}$ mass region, shown in fig. 3.11(d). No enhancement in our data can be seen anywhere except at the $\Lambda_{c}^{+}$mass.

As an additional step, the subset of the data in which the $K_{S}^{0}$ has $S F L>0.5 \mathrm{~cm}$ was analysed. The combinatoric background is reduced and the $\Lambda_{c}^{+}$signal to noise is enhanced, as expected for a particle with a relatively hard momentum spectrum. There is no evidence for any additional states. 

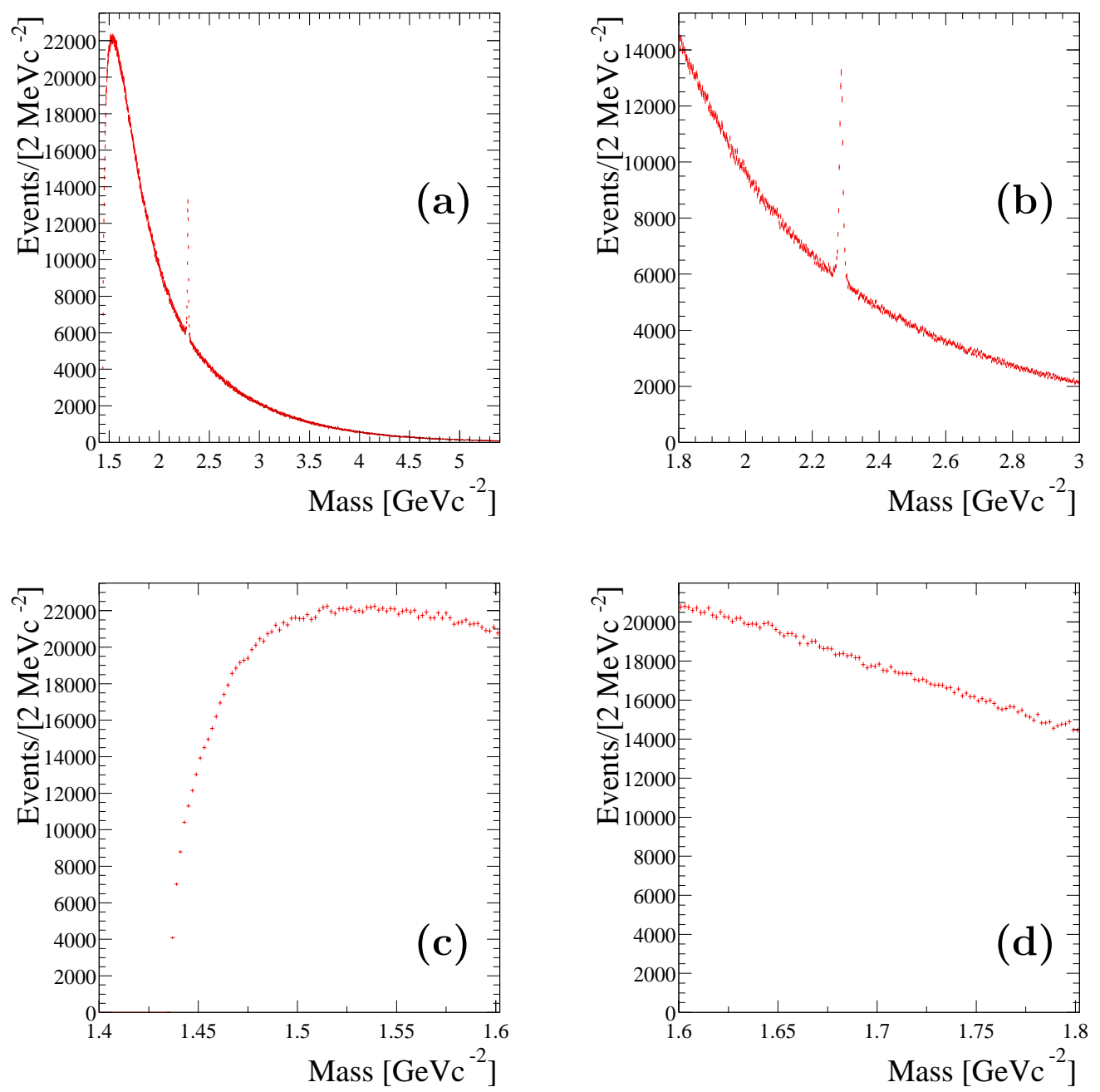

Figure 3.11: Distribution of the $p K_{S}^{0}$ invariant mass for candidates satisfying all the criteria noted in the text. The same data are plotted four times in different mass regions.

If a pentaquark is produced in $e^{+} e^{-}$annihilation, then there may also be an antibaryon (or anti-pentaquark) in the associated event, whose decay products include either a $\bar{p}$ or an anti-neutron. In the case of the $\Theta_{5}^{+}$decaying to $p K_{S}^{0}$, the $K_{S}^{0}$ must have been a $K^{0}$ rather than a $\bar{K}^{0}$, and there must be a compensating particle in the event with strangeness $=-1$, which might be expected to be a $K^{-}$rather often.

Additionally, candidates are also selected with at least one loosely identified $K^{-}\left(K^{+}\right)$ in the event, where the charged kaon is identified according to the criteria of "kLHLoose" one of the BABAR standard particle ID algorithms. This algorithm takes all the DIRC information (measured and expected number of photons, momentum, Cherenkov angle etc.) as well as $d E / d x$ into account. Further selection criteria are applied. At least one $p(\bar{p})$ track satisfying the same criteria as the $\bar{p}(p)$ in the pair is required. In both cases the data quantity is reduced and the $\Lambda_{c}$ signal is still visible. In neither case is there any sign of a pentaquark in the mass spectrum. Every combination of recoil kaon, recoil proton and signed flight length cut has been checked, yielding no evidence for a possible 
structure in the mass region where pentaquark signals have been previously claimed. For completeness, wrong-sign recoils were also considered, yielding in each case a null result.

The CLAS experiment [41] has shown evidence that the $\Theta_{5}^{+}(1540)$ is produced in the exclusive reaction $\gamma p \rightarrow \pi^{+} N^{*}(2400)$, where the excited nucleon $N^{*} \rightarrow K^{-} \Theta_{5}^{+}(1540)$ and $\Theta_{5}^{+}(1540) \rightarrow n K^{+}$. NA49 [80] have tried to reconstruct the $N^{*}(2400) \rightarrow K^{-} K_{S}^{0} p$; although there is no clear $N^{*}$ peak, when they consider the $K^{-} K_{S}^{0} p$ mass spectrum in the window $2300-2500 \mathrm{MeV} / c^{2}$, there is a more convincing enhancement in the mass spectrum for the $\Theta_{5}^{+}(1540) \rightarrow n K^{+}$than in the side bands. Although fig. 3.1 suggests that an $N^{*}$ of this mass would have a very low production rate in our data, the method of NA49 is applied to candidates with a recoil kaon. Figure 3.12, shows the result of this method. No signal in the $N^{*}$ mass region is seen, and very smooth $p K_{S}^{0}$ mass distributions are present in the sidebands. Requiring in addition a recoil baryon yields similar results with reduced statistics.
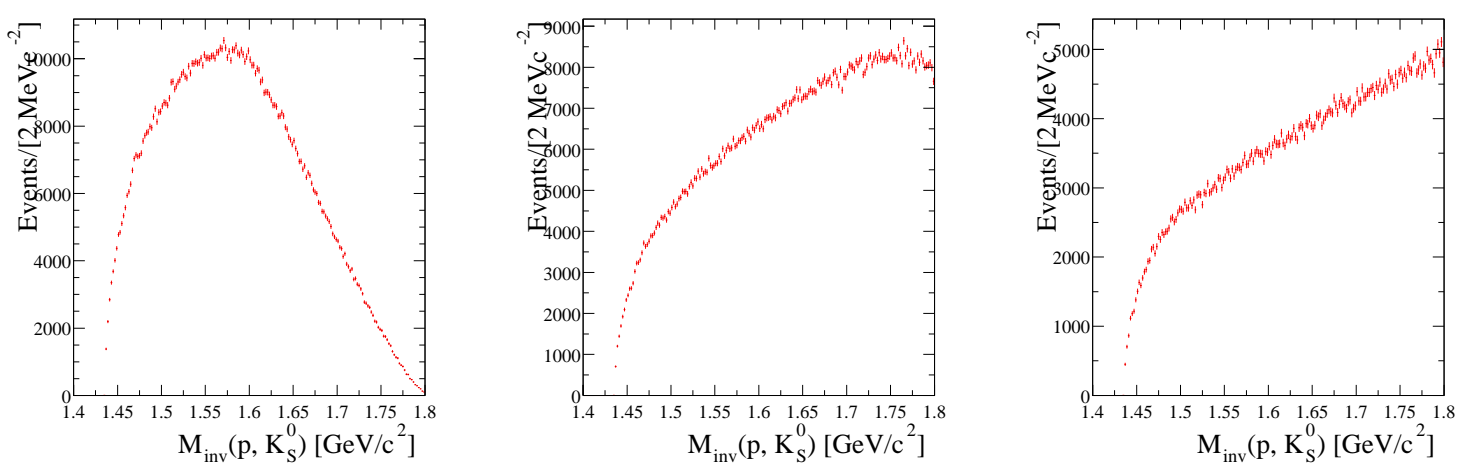

Figure 3.12: The distributions in the $K_{S}^{0} p$ invariant mass, $\mathrm{M}_{\mathrm{inv}}\left(K_{S}^{0} p\right)$, corresponding to the intervals $2.1 \rightarrow 2.3,2.5 \pm 0.1,2.5 \rightarrow 2.7 \mathrm{GeV} / c^{2}$ of $M_{\text {inv }}\left(p K_{S}^{0} K^{-}\right)$, respec. (c.f. NA49)

In each of the above cases, the data can be split into bins of $p^{*}$ in order to enhance our sensitivity to production mechanisms that give a peaked momentum spectrum. For the selection of fig. 3.11, the corresponding $\mathrm{M}\left(p K_{S}^{0}\right)$ and $\mathrm{M}\left(K_{S}^{0}\right)$ distribution can be seen in bins of $p^{*}$ of the $p K_{S}^{0}$ system in figs. 3.13 and 3.14 respectively. The combinatoral background is much smaller at higher $p^{*}$, and therefore more sensitive to mechanisms that produce harder spectra. In no case was there any sign of a pentaquark signal. An upper limit is then extracted. 

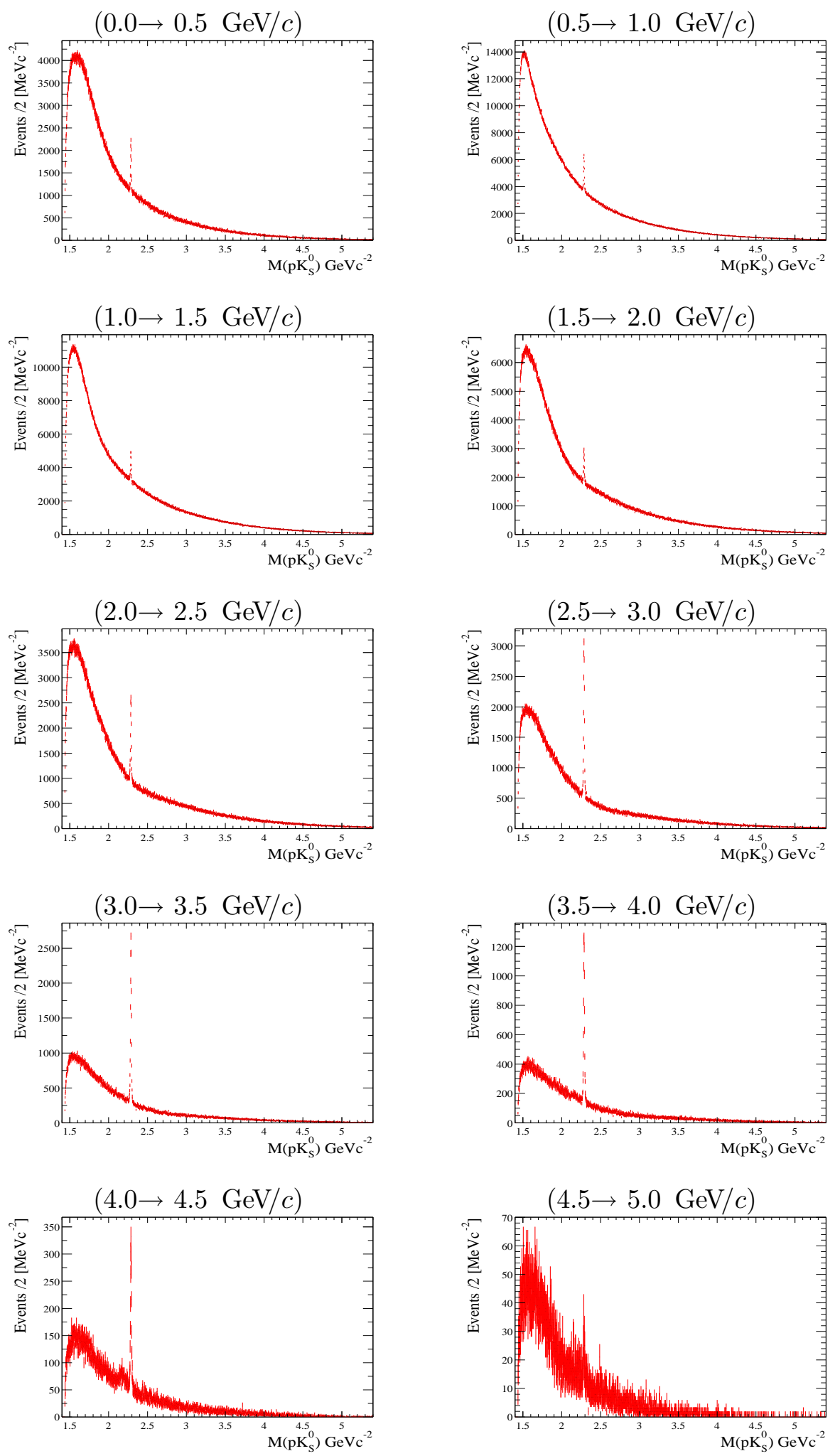

Figure 3.13: Distributions for $\mathrm{M}\left(p K_{S}^{0}\right) \mathrm{GeV} / c^{2}$ each plot represents a step of $0.5 \mathrm{GeV} / c$ in the center-of-mass momentum of the $0.0 \rightarrow 5.0 \mathrm{GeV} / c p K_{S}^{0}$ system $(\mathrm{SFL} \geq 0.0 \mathrm{~cm}$ ). 

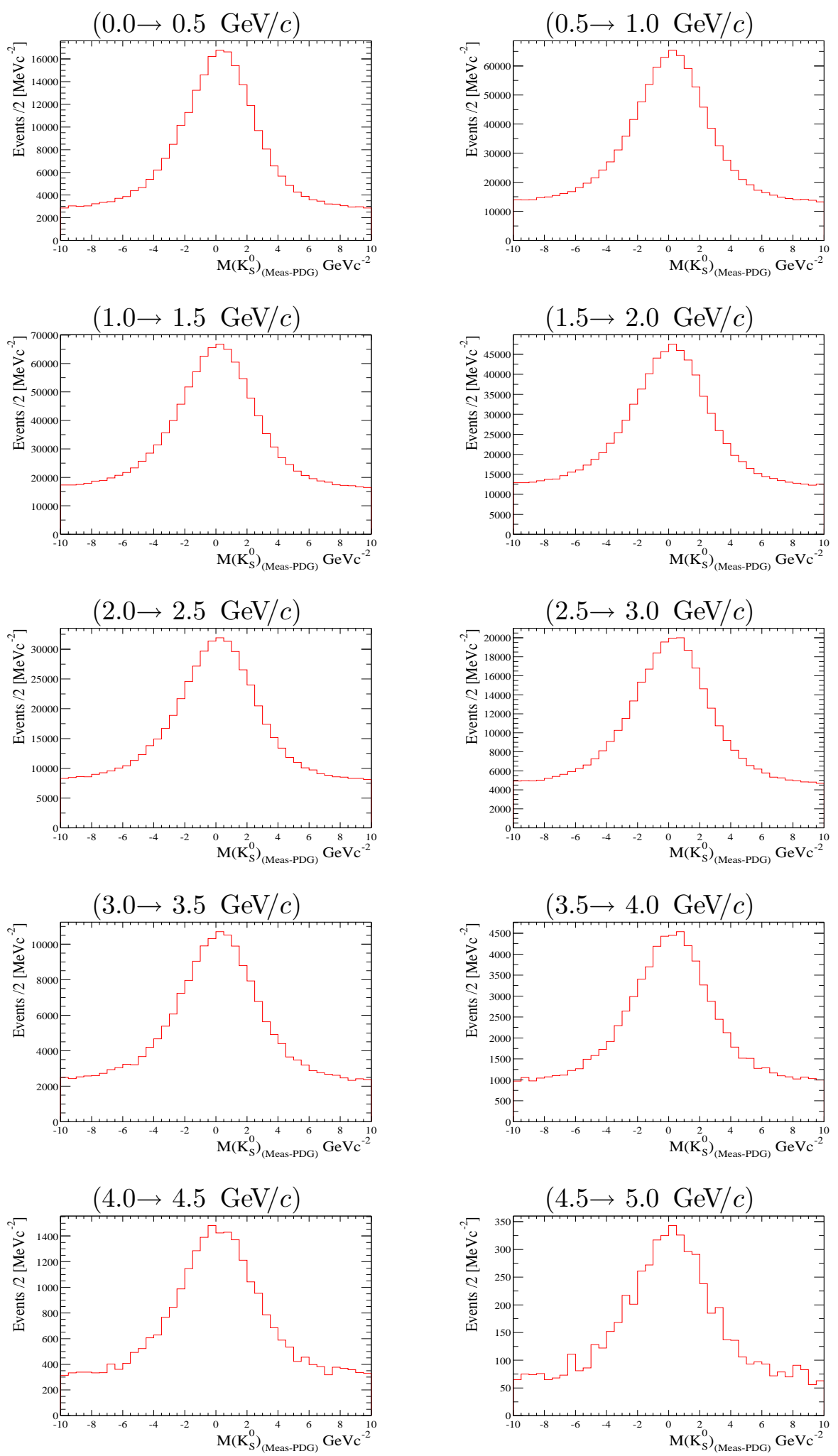

Figure 3.14: Distributions for $\mathrm{M}\left(K_{S}^{0}\right.$ meas $\left.-P D G\right) \mathrm{GeV} / c^{2}$, each plot represents a step of 0.5 $\mathrm{GeV} / c$ in the center-of-mass momentum of the $0.0 \rightarrow 5.0 \mathrm{GeV} / c p K_{S}^{0}$ system $(\mathrm{SFL} \geq 0.0$ $\mathrm{cm})$. 


\subsection{Monte Carlo Samples}

Standard Monte Carlo samples are used to study the backgrounds and evaluate the efficiencies for reconstructing "control" particles - known resonances with decay modes the same as or similar to the pentaquark in question. These controls were also used to check the invariant mass resolutions and biases of the detector, an essential ingredient in the evaluation of limits where no signal is seen.

Pentaquark Monte Carlo samples are simulated using the JETSET [81] generator. JETSET does not generate pentaquarks; although it does generate diquarks, it has no way to combine more than one di-quark. Therefore in order to simulate a pentaquark in the $\mathrm{MC}$ an existing baryon, the $\Delta^{+}(1232)$, is used as a stand-in, with its mass and width changed to match the desired pentaquark properties, and forced to decay into $p K_{S}^{0}$. MC continuum events were generated and events retained containing at least one of the standin particles. The production mechanism for such five-quark states is not known. However, to make the measurement only the efficiency and acceptance as a function of momentum are needed. Effects such as the multiplicity and proximity of other tracks in the event are difficult to study by using different existing baryons for pentaquark substitutes, as the quark content of the event is no longer balanced because JETSET is treating the stand in as a "normal" three quark object; however such effects are generally much smaller than the other systematic uncertainties.

The simulated efficiencies for $\Theta_{5}^{+}$and the $\Lambda_{c}^{+}$from $c \bar{c}$ events are shown as a function of $p^{*}$ (the total momentum of the $p K_{S}^{0}$ pair boosted back into the c.m. frame) in fig. 3.15. The two samples are consistent at higher $p^{*}$, however at low $p^{*}$ the distributions differ significantly due to the mass of the $\Lambda_{c}^{+}$being larger than that of the $\Theta_{5}^{+}$, therefore the decay products have higher momentum in the center of mass system, due to the energy transferred from the mother particle.

\subsection{Cross Sections and Upper Limits}

Since the production mechanism, and hence the momentum spectrum, for pentaquarks is unknown, measurements of, or limits upon the differential cross section, $d \sigma / d p^{*}$, are obtained per unit $e^{+} e^{-}$center of mass momentum. The procedure is to divide each set of candidates into bins of $p^{*}$ and fit an appropriate function to the invariant mass distribution in each bin. The area under the fitted signal is an estimate of the observed number of true particles, $N$, and the differential cross section is given by,

$$
d \sigma / d p^{*}=\frac{N}{\epsilon \times L \times B \times \Delta p^{*}}
$$

where $\epsilon$ is the signal efficiency, $L$ is the integrated luminosity, $B$ is the branching fraction into the reconstructed mode and $\Delta p^{*}$ is the bin width.

If the signal is consistent with zero, a differential limit on the differential production 


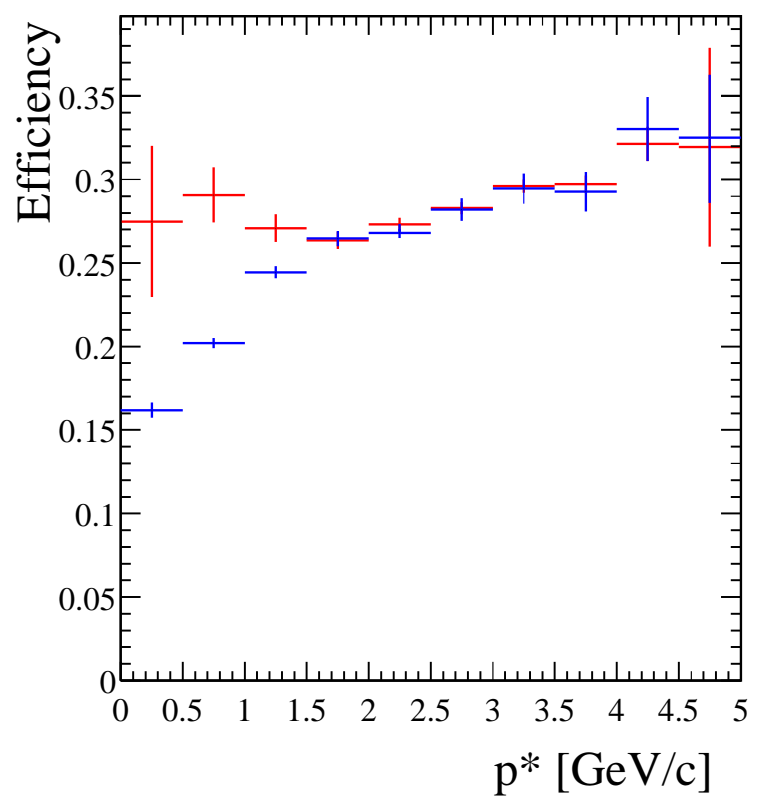

Figure 3.15: Measured reconstruction efficiencies as a function of $p^{*}$; the $\Lambda_{c}^{+} \rightarrow p K_{S}^{0}$ from $c \bar{c} \mathrm{MC}$ is shown in red; the $\Theta_{5}^{+}$is shown in blue.

cross section can be derived as a function of $p^{*}$ using:

$$
d \sigma / d p^{*}<\frac{N^{\max }}{\epsilon \cdot L \cdot B \cdot \Delta p^{*}}
$$

where $N^{\max }$ is the $95 \%$ C.L. upper limit on the raw number of signal events in the data. Since the statistics of the background are very high in all cases, the observed number of events is expected to be distributed as a Gaussian, so limits can be set in the usual way based upon the standard deviation $\Delta N$ of this Gaussian. The value of $\Delta N$ is taken to be sum in quadrature of the statistical error returned from the fit described in section 3.8.1 and the systematic error given in table 3.2. Since the true value cannot be negative, two methods of estimating $N^{\max }$, with the constraint that it must positive, are considered. These are described in section 3.8.1. Such a limit can be converted into a limit on the multiplicity per event of a given type, if the cross section for that event type is known; $e^{+} e^{-} \rightarrow q \bar{q} \rightarrow$ hadrons $(q=u d s c)$ and $\Upsilon(4 S)$ decays can be considered. A tighter limit on each production type is possible if the types can be separated in a clean and unbiased fashion, but in this case the best limits come from simply taking all events together.

Note that when a limit on a cross section as a function of momentum is calculated, it is to first order a model independent limit applicable to the sum of all processes in $e^{+} e^{-}$ interactions at $\sqrt{s}=10.58 \mathrm{GeV}$. There are higher order effects that can bring in some model dependence, e.g. different production $\cos \theta$ distributions of pentaquarks, entering through fiducial efficiency loss, or fast cross section variation at low momentum on the scale of the momentum bins, etc., but this is believed to be small. The integral of this 
cross section is thus an unbiased, model independent measurement of the total production rate, from which one can derive a conservative, model independent upper limit on the total rate, providing a useful constraint on many models. If a production model predicts a spectrum, it can be folded with the obtained differential constraint to obtain a tighter limit on that particular model.

In order to set an upper limit on the cross section the signal line shape, detector resolution function, including any mass shifts, and the signal reconstruction efficiency must be known. These are established in part by measuring the cross section for the $\Lambda_{c}^{+}$ baryon decaying to the $p K_{S}^{0}$ final state, which has known mass and width and is clearly visible in the data. 


\subsubsection{The $\Lambda_{c}^{+}$Differential Cross Section}
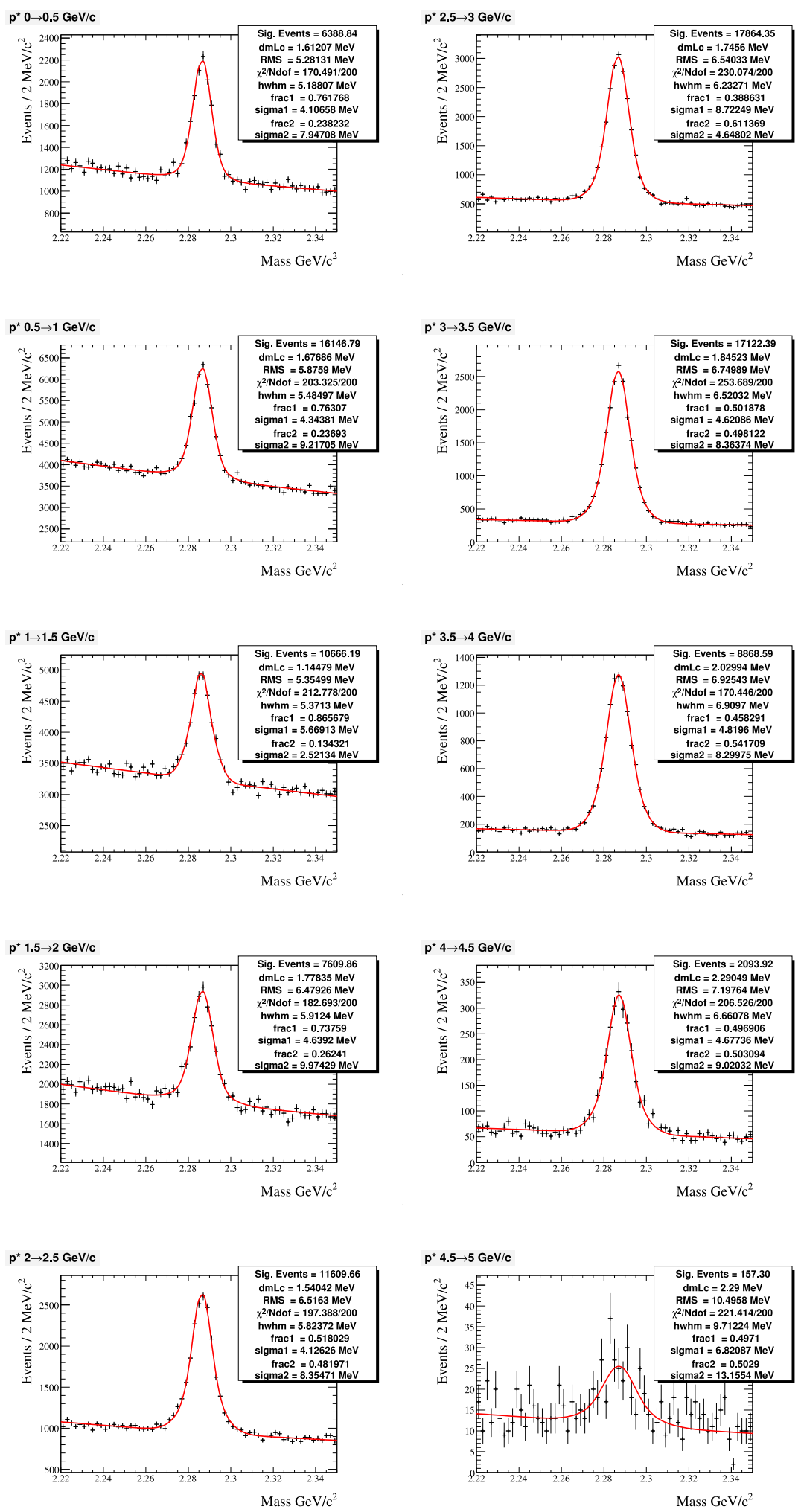

Figure 3.16: Distribution of the $p K_{S}^{0}$ invariant mass in different $p^{*}$ bins in the region of the $\Lambda_{c}^{+}$. The lines represent the fits described in the text.

The selected $p K_{S}^{0}$ combinations are divided into $0.5 \mathrm{GeV} / c$ bins of $p^{*}$, and the $p K_{S}^{0}$ 
mass distribution in each bin is considered, shown in fig. 3.16. A function is fitted to each of these distributions to extract a signal. Since the $\Lambda_{c}^{+}$decays weakly, it has essentially zero width, and the signal function in the fit is simply the detector resolution function. Based upon fits to the corresponding MC, this is taken to be a double Gaussian and the background is represented by a 2nd order polynomial, except in the first and last bin where 3rd and 1st order polynomials are used, respectively. Fits with a good $\chi^{2}$ are observed in all cases, as indicated by the curves on fig. 3.16. The fitted mass values are shown in fig. 3.17(a) and are compared with the PDG value; they are within $2.3 \mathrm{MeV} / \mathrm{c}^{2}$ at all momenta ${ }^{5}$. The fitted widths, also shown in fig. 3.17(b), are consistent with MC predictions, dominated by detector resolution. Systematic effects arising from the fitting procedure are considered, by varying the background and signal shapes, fixing the masses and/or widths to PDG values, etc. This leads to variations in the results which are much smaller than the errors on the efficiency, hence these effects are therefore neglected.

The efficiency for reconstructing true $\Lambda_{c}^{+} \rightarrow p K_{S}^{0}$ is derived from $c \bar{c} \mathrm{MC}$, and corrected for known deficiencies in the track-finding and particle identification efficiencies. For charged particles extrapolating close to the $z$-axis of the detector there is an official BABAR
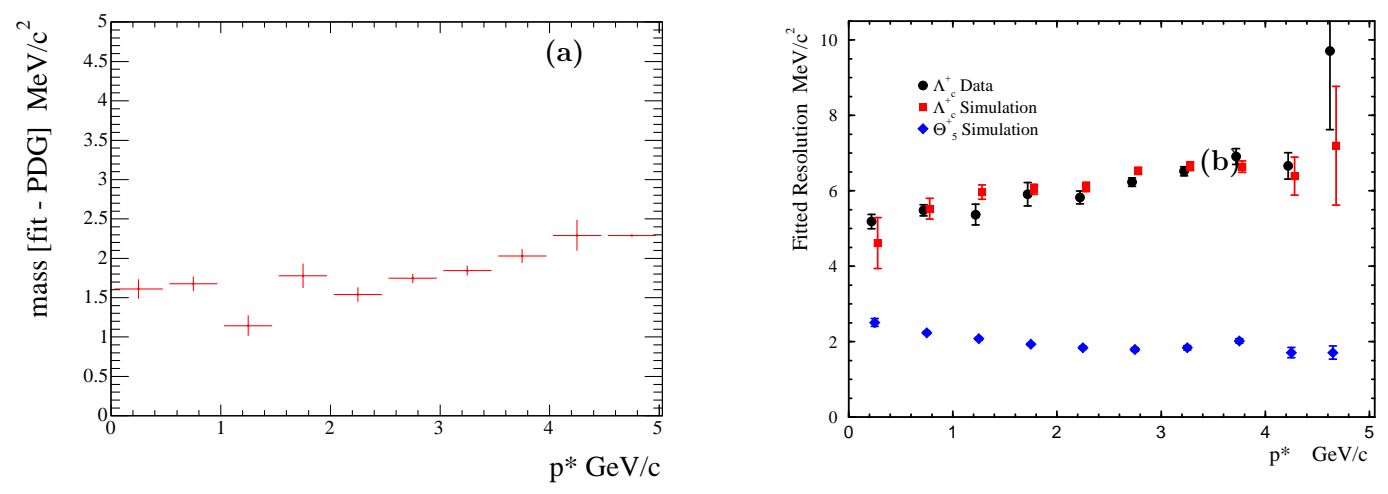

Figure 3.17: Fitted mass values (a) for the $\Lambda_{c}^{+}$signal in the data minus the PDG value as a function of $p$. Fitted widths (b) of the detector resolution functions for data (circles) and MC (open circles)

correction [82] to the track finding efficiency for this reconstructed dataset of $-0.8 \pm 1.4 \%{ }^{6}$, that is independent of momentum, polar angle and particle type. This correction is applied to the proton track efficiency, with the uncertainty taken as a systematic error. Except for the proton identification, the effect of the other cuts is minimal for protons from true $\Lambda_{c}^{+}$ decay, and consistency is found between data and MC, so no further corrections or errors are considered. The proton identification efficiency has been evaluated for the selection chosen here using the prescription and ntuples provided by the PID working group. The

\footnotetext{
${ }^{5}$ Brian Petersen, Stanford Univ., private communication: the shift in the measured $\Lambda_{c}^{+}$mass from the "accepted" PDG value is not unexpected, the most up-to-date measurement of this was presented at APS 2005, Prelim. result.

${ }^{6}$ The actual correction for efficiency is for tracks satisfying $1.5 \mathrm{~cm}$ POCA in the XY direction, and $\pm 10 \mathrm{~cm}$ in the POCA in the $\mathrm{Z}$ direction, to the $\mathrm{Z}$-axis of the detector, the selection is inside this region therefore and is therefore covered by this procedure.
} 
systematic uncertainty is evaluated by calculating the ratio of data to MC in each interval of proton lab. momentum and calculating the weighted mean of the resulting values. The uncertainty was estimated to be the r.m.s. deviation of the individual ratio values with respect to the mean. The estimated correction factor obtained in this way is $0.98 \pm 0.02$ (see fig 3.18(b)).
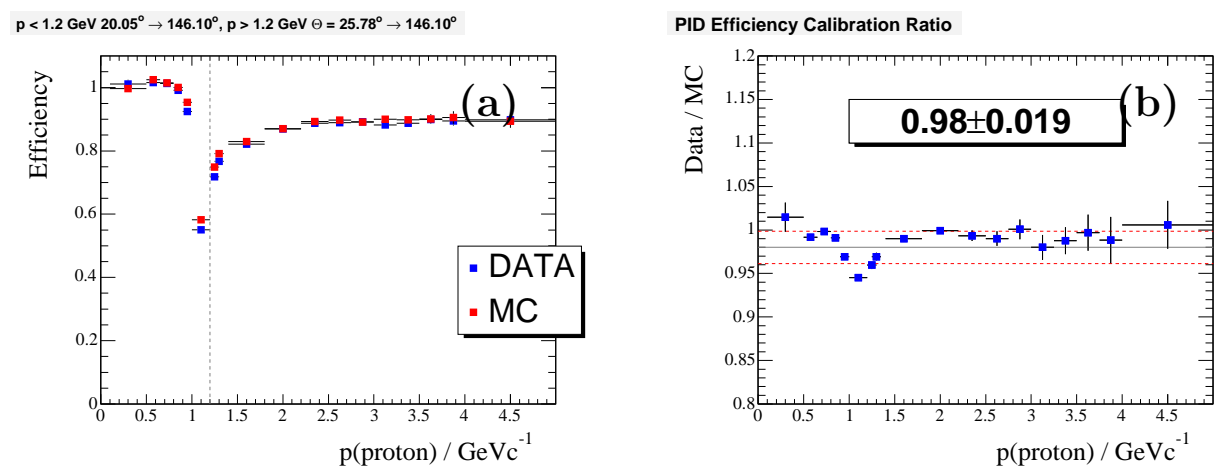

Figure 3.18: (a) The proton PID efficiency distribution for data and MC for the momentum and angular distributions considered; (b) the ratio of data to $\mathrm{MC}$ efficiency.

For charged pions from $K_{S}^{0}$ decays, there is a larger recommended correction of $-1.5 \pm 1.6 \%$ [82], which has been applied to both the $\pi^{+}$and the $\pi^{-}$track. This correction has been evaluated for $K_{S}^{0}$ candidates reconstructed with a specific set of cuts, which correspond to the selection before any of the selection criteria given in sec. 3.5.1. The effect of the other requirements has been studied, and it is found that none of them rejects any significant signal. The cut on the DOCA of the two daughter tracks does remove about $1 \%$ of the true $K_{S}^{0}$ in the MC; however these are poorly reconstructed due mainly to decays in flight of the pions and do not give good mass measurements. It is assumed that the MC models this small loss correctly. The pion particle identification is very loose, and no correction is needed. These corrections are summarized in table 3.2. The total systematic error on the efficiency is about $6 \%$ for both the $\Lambda_{c}^{+}$and potential $\Theta_{5}^{+}(1540)$ signals; they are essentially entirely normalization efficiencies and do not depend on $p^{*}$.

Table 3.2: Corrections and systematic errors, that are applied to the tracks from the $p K_{S}^{0}$ candidates.

\begin{tabular}{l|c} 
Source & Correction \\
\hline \hline$\pi^{+}$track & $0.985 \pm 0.016$ \\
$\pi^{-}$track & $0.985 \pm 0.016$ \\
\hline proton track & $0.992 \pm 0.014$ \\
proton PID & $0.980 \pm 0.019$ \\
\hline Total & $0.943 \pm 0.033$
\end{tabular}

The simulated efficiency is shown as a function of $p^{*}$ in fig. 3.15, and is high over the full kinematic range. The number of signal events is divided by this efficiency, the integrated luminosity, and the branching fraction $B\left(\bar{K}^{0} \rightarrow \pi^{+} \pi^{-}\right)(=0.3448$ [9]) to yield 
the cross section integrated over each $p^{*}$ interval, which is shown in fig. 3.19. This shows a concentration at high $p^{*}$ values due to leading particles in $c \bar{c}$ events and another at low $p^{*}$ due to $B$ meson decays, as expected. Comparing this measurement with that measured using the $p K^{-} \pi^{+}$decay mode [83], consistency in both shape and normalization is found. The sum of the total cross-section for $\Lambda_{c}+\overline{\Lambda_{c}}$ production after correcting for the branching fraction $B\left(\Lambda_{c} \rightarrow p K_{S}^{0}\right)[9]$ is estimated to be $380 \pm 100 \mathrm{pb}$.

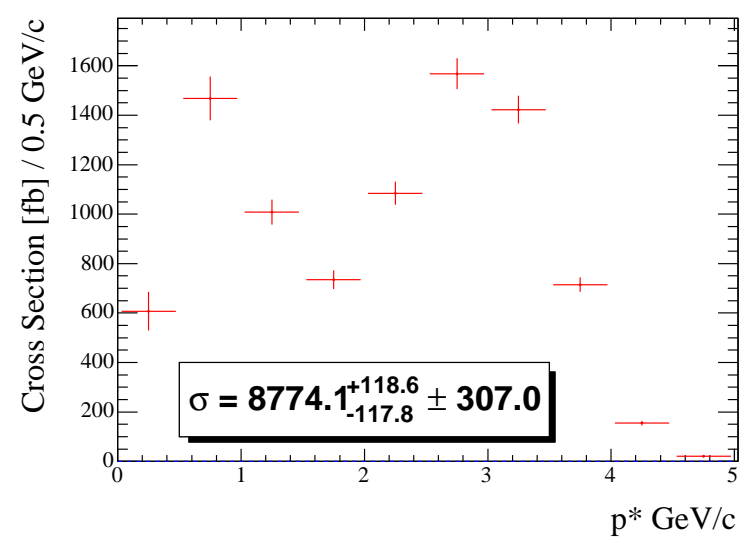

Figure 3.19: Corrected $\Lambda_{c}^{+}$production cross section as a function of $p^{*}$. This shows a concentration at high $p^{*}$ values due particles in $c \bar{c}$ events and another at low $B$ meson decays, as expected.

\subsubsection{Estimating 95\% C.L. Limits on the $\Theta^{+}$Cross Section}

In order to set upper limits on possible pentaquark production, the selected $p K_{S}^{0}$ pairs are first divided into $p^{*}$ bins, and a signal plus background function is fitted to the $p K_{S}^{0}$ invariant mass distribution in each bin, as per the cross section measurement. The natural width of the $\Theta_{5}^{+}$has not been measured, and the best upper limit on the width, $8 \mathrm{MeV} / \mathrm{c}^{2}$, is larger than the detector resolution, therefore several possibilities up to this value are considered.

\section{Fit Procedure and Error Estimates}

The fit procedure uses a $\mathrm{P}$-wave Breit-Wigner lineshape, multiplied by a phase space factor, smeared by a resolution function derived from the $\mathrm{MC}$, and normalised such that the integral over this net shape corresponds to one event. The resolution function consists of two Gaussian distributions with a common center, as used to describe the $\Lambda_{c}$. However, as the $\Theta_{5}^{+}$region is much closer to the $K_{S}^{0} p$ threshold, i.e. the Q-value, given by $\left(m\left(K_{S}^{0} p\right)-\right.$ $\left.m_{\text {thresh }}\right)$, is much smaller, the mass resolution is significantly better than that at the $\Lambda_{c}$ mass, ranging from $\sim 2.5 \mathrm{MeV} / \mathrm{c}^{2}$ at low $p^{*}$ to $\sim 1.8 \mathrm{MeV} / \mathrm{c}^{2}$ at high $p^{*}$ (see fig3.17(b)).

For the natural width, the values $1 \mathrm{MeV}$, which is $\sim 5$ times narrower than our resolution, and $8 \mathrm{MeV}$ which is the upper limit from experiment are considered. With 
these choices, the upper limit estimates span the range of probable width values. The corresponding functions are shown in fig. 3.20. The Breit-Wigner tails extend over a broad mass region.
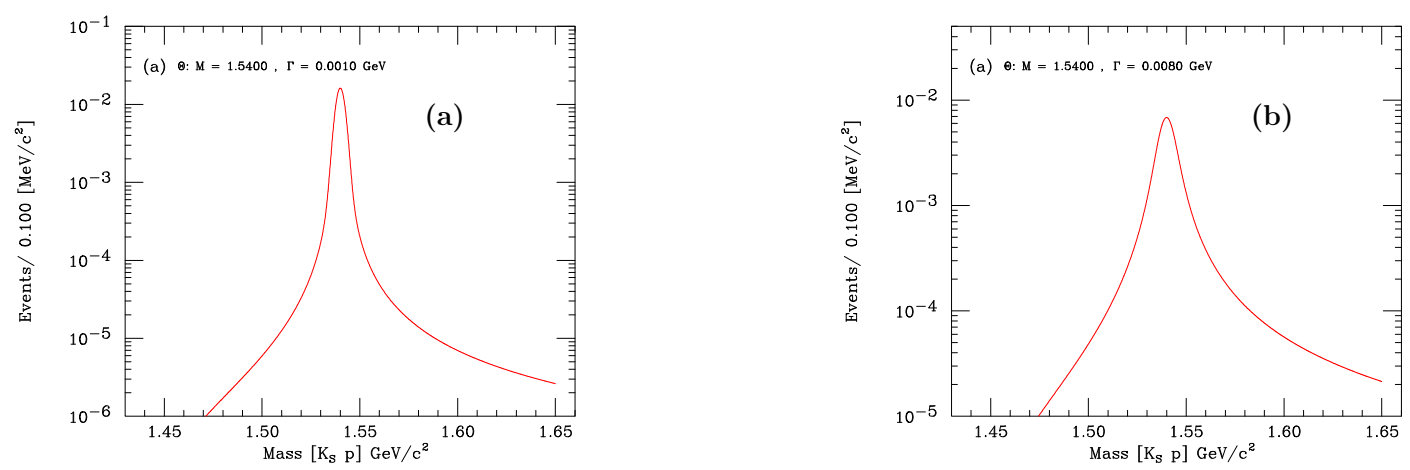

Figure 3.20: The signal function used in the fits for the $\Theta_{5}^{+}$assuming a natural width of (a) 1 or (b) $8 \mathrm{MeV} / \mathrm{c}^{2}$.

Since the $\Theta_{5}^{+}$is rather close to threshold, fits are performed over the range from threshold to $1800 \mathrm{MeV} / \mathrm{c}^{2}$, in order to obtain a stable background estimate in the region of the $\Theta_{5}^{+}$. The background is represented by a function of the form:

$$
q \times\left\{7^{\text {th }} \text { order polynomial in }\left(m-m_{\text {thresh }}\right)\right\}
$$

where $m$ is the mass of the $K_{S}^{0} p$ system, $m_{\text {thresh }}$ its threshold value, $1436 \mathrm{MeV} / c^{2}$, and $q$ is the magnitude of the 3 -momentum vector in the $K_{S}^{0} p$ rest frame. This latter factor results from the 2-body phase space, $q / m$, multiplied by the factor $m$, due to the fact that the Lorentz-invariant phase space volume element $d(L I P S)$ contains $d\left(m^{2}\right)$, not $d(m)$. The order of the polynomial is the lowest for which a fit excluding the signal region gives a good $\chi^{2}$ probability for the entire range. In no case does the fitted polynomial exhibit rapid fluctuation anywhere in the fit region. 

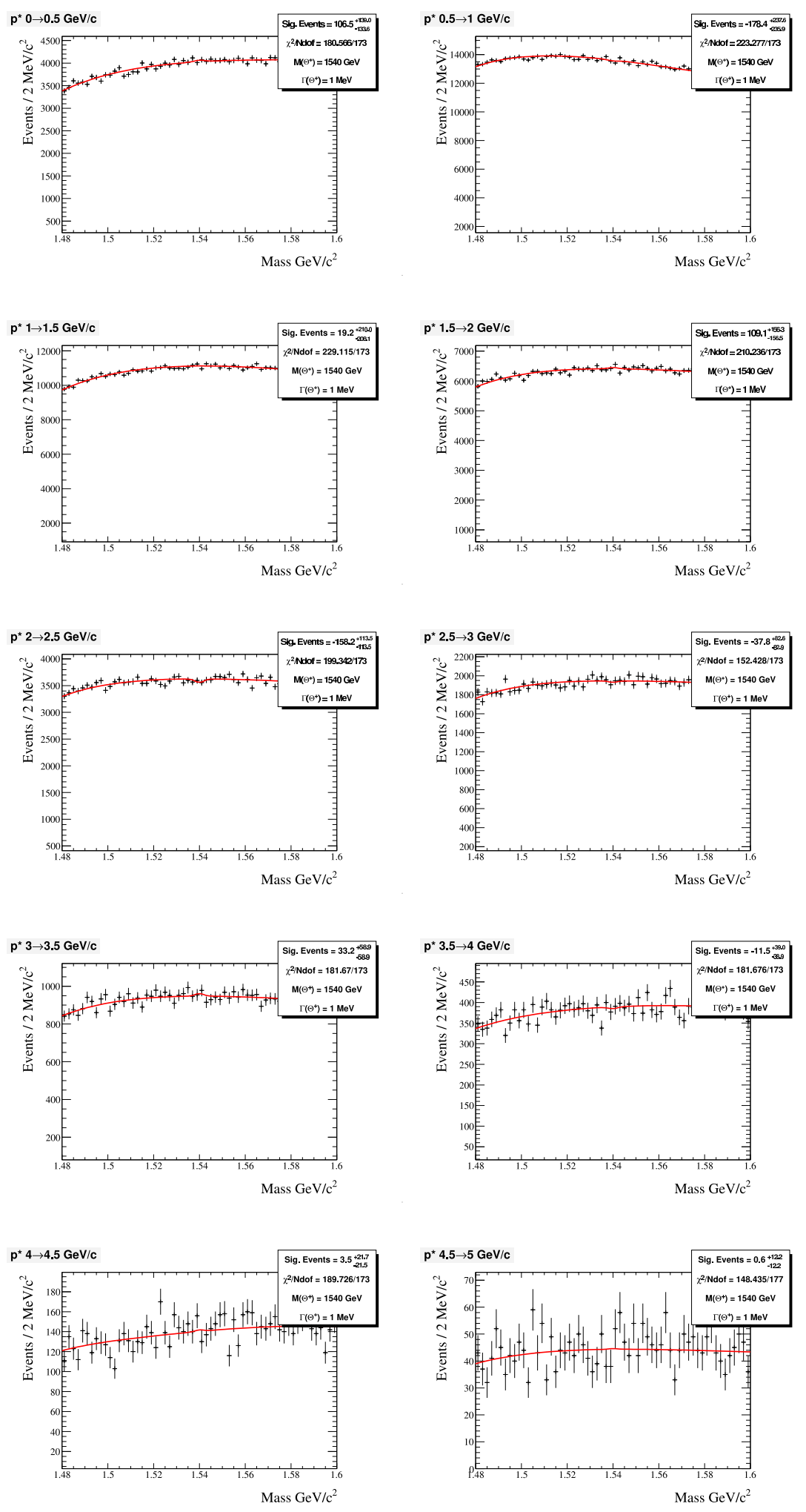

Figure 3.21: The $p K_{S}^{0}$ invariant mass distribution in each $p^{*}$ bin; the lines represent the results of the fit described in the text. 
First the central mass is fixed to $1540 \mathrm{MeV} / \mathrm{c}^{2}$ and the invariant mass distributions are fitted with a smeared signal function corresponding to $\Gamma=1 \mathrm{MeV}$ as shown in fig. 3.21. In all cases the fit quality is good and the $\Theta_{5}^{+}$signal is consistent with zero. By construction, the coefficient of the signal function obtained from the fit corresponds to the estimated signal size; this is shown in fig. 3.22 as a function of $p^{*}$. In all cases the signal is consistent with zero. The uncertainty in the signal size is obtained by performing a MINOS [84] search. The positive and negative one standard deviation error values, $\sigma_{+}$and $\sigma_{-}$, are found to differ only slightly for the fits in question, and so the $\chi^{2}$ surface is considered to be approximately paraboloid in the vicinity of the minimum for the purpose of estimating 95\% C.L. limits. The separate values, $\sigma_{+}$and $\sigma_{-}$, however are used as explained below.

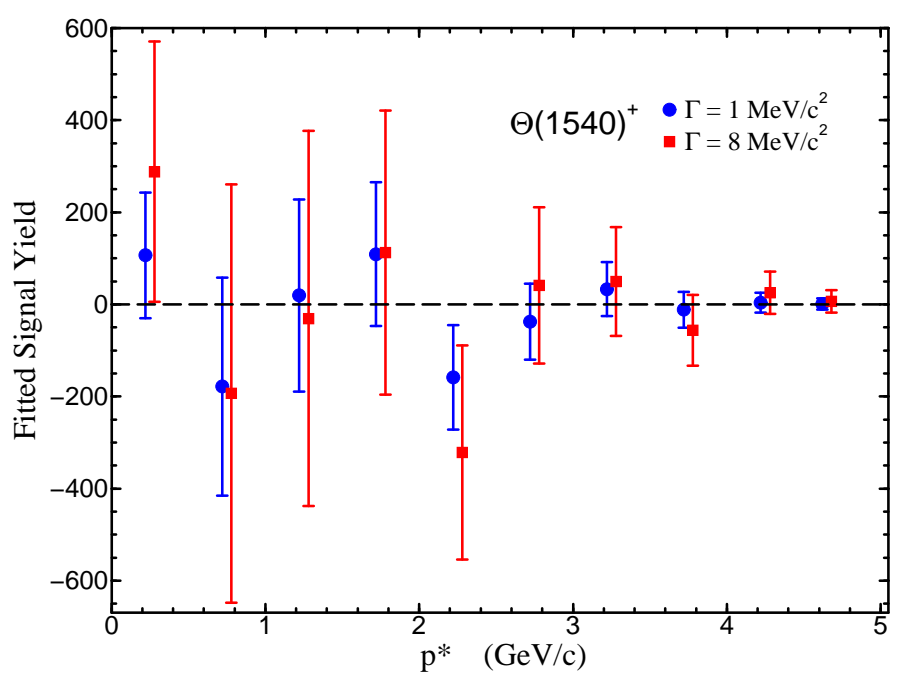

Figure 3.22: The signal extracted from the fits for the $\Theta_{5}^{+}$assuming a mass of $1540 \mathrm{MeV} / \mathrm{c}^{2}$ and natural width of 1 (left) and $8 \mathrm{MeV} / c^{2}$ (right).

\section{Signal Fit Size Negative or Zero}

In this case, the $95 \%$ c.l. limit is estimated by centering the signal at zero and considering the region of positive values to have a Gaussian probability distribution with standard deviation $\sigma_{+}$.

The signal value, $x_{0}$, such that the integral of the Gaussian from 0 to $x_{0}$ yields an area which is $0.95 \times 0.5$ is then

$$
x_{0}=1.96 \times \sigma_{+}
$$

and this is used as the $95 \%$ c.l. upper limit estimate in this case.

\section{Signal Fit Size Positive}

If the signal value is $m$, the probability distribution is considered to be centered at $m$, and described by a Gaussian of standard deviation $\sigma_{-}$truncated at 0 on the low side, and by 
a Gaussian of standard deviation $\sigma_{+}$on the high side.

The $95 \%$ c.l. limit estimate, $x_{0}$, is defined to be such that the integral of this truncated function from 0 to $x_{0}$ is equal to $95 \%$ of the value of this same integral from 0 to infinity.

Let $a_{1}$ be the area from 0 to the signal value $m$.

Let $a_{2} x_{0}$ be the area from $m$ to $x_{0}$, where $x_{0}>m$.

Since the total area for values greater than $m$ is 0.5 , by construction, it follows that $x_{0}$ is the solution of the equation

$$
a_{1}+a_{2} x_{0}=0.95 \times\left(a_{1}+0.5\right)
$$

i.e.

$$
a_{2} x_{0}=0.95 \times 0.5-0.05 \times a_{1}=0.475-0.05 \times a_{1}
$$

From the definition of the error function, $\operatorname{erf}^{7}$,

$$
a_{1}=0.5 \times \operatorname{erf}(u)
$$

where

$$
u=\left[m /\left(\sigma_{-} \sqrt{ } 2\right)\right]
$$

so that the right-hand side of equation[3.13] can be evaluated in a straightforward way.

Similarly,

$$
a_{2} x_{0}=0.5 \times \operatorname{erf}(u)
$$

where

$$
u=\left[\left(x_{0}-m\right) /\left(\sigma_{+} \sqrt{ } 2\right)\right]
$$

and the value of $x_{0}$ is obtained by stepping the value and evaluating [3.16] until [3.13] is satisfied.

The maximum value of $a_{1}$ is 0.5 , so that the minimum value of $x_{0}$ is such that (from [3.13])

$$
a_{2} x_{0}=0.45
$$

${ }^{7}$ Computation of the error function

$$
\operatorname{erf}(x)=[2 / \sqrt{ } \pi] \int_{0}^{x} \exp \left(-t^{2}\right) d t
$$

For a Gaussian function normalized to unit area, the area for values greater than the mean, which is 0.5 , corresponds to

$$
0.5 \times \operatorname{erf}(\infty)
$$

and the area from the mean value, $m$, to $x$ is given by

$$
0.5 \times \operatorname{erf}(u)
$$

where:

$$
u=[(x-m) /(\sigma \sqrt{ } 2)]
$$


For a full Gaussian this represents $90 \%$ c.l., so that the corresponding value of $x_{0}$ is

$$
x_{0}=m+1.64 \times \sigma_{+}
$$

The minimum value of $a 1$ is 0 , and in this case

$$
x_{0}=m+1.96 \times \sigma_{+}
$$

as discussed above for negative or zero signal.

It follows that the numerical search for the $95 \%$ c.l. value of $x_{0}$ is restricted to the range

$$
1.64 \times \sigma_{+}<\left[x_{0}-m\right]<1.96 \times \sigma_{+}
$$

and so the value which satisfies eqn.[3.13] is readily found.

\section{Alternative Treatment When Mean Value is Negative}

If the signal value is negative, say $-m$ with $m$ positive, the probability distribution is considered to be centered at $-m$, and described by a Gaussian of standard deviation $\sigma_{+}$ towards positive values. The region of interest is then considered to be the part of this Gaussian in the range of positive signal values.

The $95 \%$ c.l. limit estimate, $x_{0}$, is defined to be such that the integral of this truncated function from 0 to $x_{0}$ is equal to $95 \%$ of the value of this same integral from 0 to infinity.

Let $a_{1}$ be the area from 0 to infinity.

Let $a_{2} x_{0}$ be the area from 0 to $x_{0}$.

From the definition of the error function, erf,

$$
a_{1}=0.5-0.5 \times \operatorname{erf}(u)
$$

where

$$
u=\left[m /\left(\sigma_{+} \sqrt{ } 2\right)\right]
$$

It follows that $x_{0}$ is the solution of the equation

$$
a_{2} x_{0}=0.95 \times a_{1}
$$

i.e.

$$
a_{2} x_{0}=0.475-0.475 \times \operatorname{erf}\left[m /\left(\sigma_{+} \sqrt{ } 2\right)\right]
$$

But

$$
a_{2} x_{0}=0.5 \times \operatorname{erf}\left[\left(x_{0}+m\right) /\left(\sigma_{+} \sqrt{ } 2\right)\right]-0.5 \times \operatorname{erf}\left[(m) /\left(\sigma_{+} \sqrt{ } 2\right)\right]
$$

so that, from $[3.26]$ and $[3.25]$

$$
\operatorname{erf}\left[\left(x_{0}+m\right) /\left(\sigma_{+} \sqrt{ } 2\right)\right]=0.95+0.05 \times \operatorname{erf}\left[m /\left(\sigma_{+} \sqrt{ } 2\right)\right]
$$


In the limiting case, $m=0$,

$$
\operatorname{erf}\left[\left(x_{0}\right) /\left(\sigma_{+} \sqrt{ } 2\right)\right]=0.95
$$

and this gives $x_{0}=1.96 \times \sigma_{+}$as before. In general, $x_{0}$ is obtained by stepping the argument in the left-hand side of [3.27] until the equality is satisfied.

For each case in which the fitted cross section is negative, the upper limit has been estimated using the procedure described above. In every instance, this limit is lower than that obtained w.r.t. a mean value of zero. This occurs as a consequence of using an unphysical fitted cross section value. Therefore, to be more conservative, the first procedure can be used obtain the upper limit estimate in such cases. However, for this analysis both results are used.

Upper limits are derived according to eqn 3.9. The efficiencies obtained from simulation are corrected as described above (see table 3.2) and are shown in fig. 3.15. The systematic uncertainty on the efficiency is propagated simply by scaling the uncertainty on the observed number of events by the factor $(1+\delta \epsilon / \epsilon)$.

Here it is more important to consider systematic effects coming from the fit to the expected signal region, than it was for the control particles. Varying the background shape has a negligible effect on the extracted signal. Variation of the width does change the limit, and this is covered by using the two width hypothesis and the upper limit value noted above, which gives a systematic range. Variation of the central mass value also has no effect above that induced by statistical fluctuations; we have verified that our choice of $1540 \mathrm{MeV} / \mathrm{c}^{2}$ is not a point at which there is a large fluctuation downward. Therefore these effects are neglected relative to the systematic on the efficiency. Since the mass of the observed state is rather uncertain, the analysis is repeated at several mass values between 1520 and $1560 \mathrm{MeV} / \mathrm{c}^{2}$; in each case limits are obtained consistent with those at $1540 \mathrm{MeV} / \mathrm{c}^{2}$, therefore placing a limit on the production of a narrow state of any mass within this range at the same level. The upper limits are shown in fig. 3.23 and discussed in the summary section.

\subsection{Summary}

A high statistics search for the reported pentaquark candidate $\Theta_{5}^{+}(1540)$ has been performed in $e^{+} e^{-}$annihilations. No evidence has been found for the production in $123.5 \mathrm{fb}^{-1}$ of $B A B A R$ data. No excess at the invariant mass values reported by previous experiments is seen and the reported limits on the width are used to set upper limits on the inclusive production rates in hadronic events. Limits on the differential production cross section have been derived in two ways, both of which assume the value cannot be negative; these are shown in fig. 3.23 and in table 3.3 for the two hypotheses regarding the width, the experimental upper limit value of $8 \mathrm{MeV}$ and a value of $1 \mathrm{MeV}$, corresponding to a very narrow state. If a positive value is measured, a Gaussian centered at the measured value can be integrated from zero to infinity, the point at which this integral reaches $95 \%$ of its 

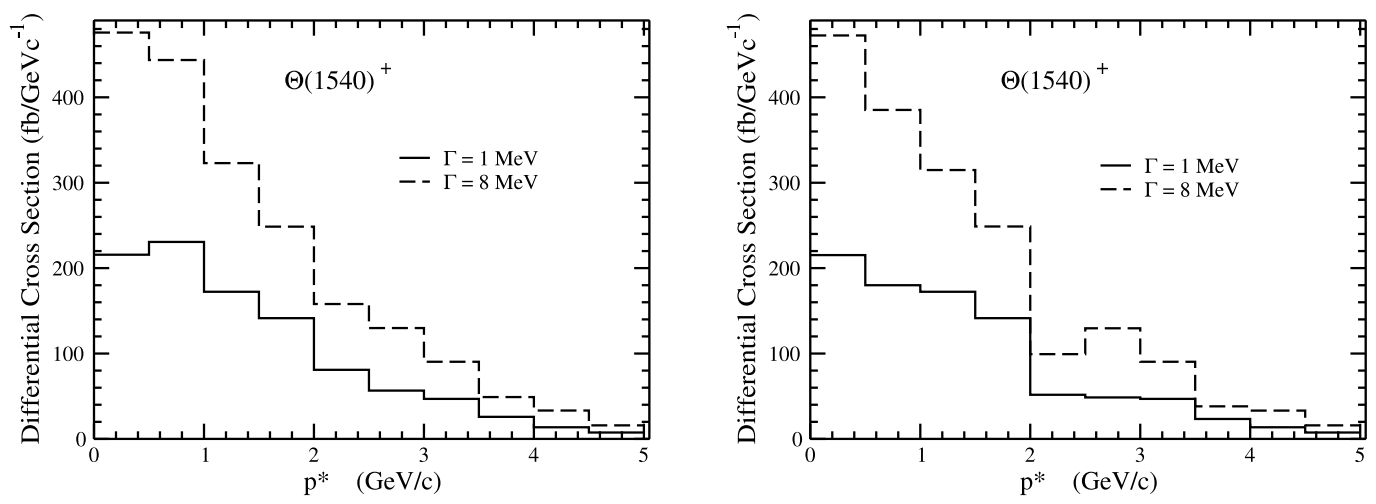

Figure 3.23: The 95\% C.L. upper limit on the $\Theta_{5}^{+}$differential production cross-section from the two methods described in the text, assuming $\Gamma=1$ (solid) and $\Gamma=8 \mathrm{MeV} / \mathrm{c}^{2}$ (dashed), and $B F\left(\Theta_{5}^{+} \rightarrow p K_{S}^{0}\right)=1 / 4$.

total can then be found. If the measured value is negative then either of the following was done: a) simply take the $1.96 \sigma$ as the limit, which has the advantage of giving a smooth differential limit, but is quite conservative in the case of a downward statistical fluctuation; or b) perform the same Gaussian integration from zero. In all cases a branching fraction to $K_{S}^{0} p$ of one-quarter is assumed, since only the decay modes $n K^{+}$and $p K_{S}^{0}$ are possible for a $\Theta_{5}^{+}$in the expected mass region.

Table 3.3: Upper limits on the differential production cross section for $\Theta_{5}^{+}$, assuming a mass of $1540 \mathrm{MeV} / c^{2}$ and a branching fraction into $p K_{S}^{0}$ of one-quarter, in units of $\mathrm{fb} / \mathrm{GeV} / \mathrm{c}$. 'A' indicates limits derived using eqns 3.12 and 3.17, and 'B' denotes those using eqns. 3.24 and 3.17. The two values of the natural width discussed in the text are used.

\begin{tabular}{|c|rr|rr|}
\hline$p^{*}$ Range & \multicolumn{2}{|c|}{$\Gamma=1$} & $\mathrm{MeV}$ & \multicolumn{2}{|c|}{$\Gamma=8$} & $\mathrm{MeV}$ \\
$\mathrm{GeV} / c$ & $\mathrm{~A}$ & $\mathrm{~B}$ & $\mathrm{~A}$ & $\mathrm{~B}$ \\
\hline $0.0-0.5$ & 215.7 & 215.7 & 475.8 & 475.8 \\
$0.5-1.0$ & 230.6 & 179.9 & 443.6 & 385.1 \\
$1.0-1.5$ & 172.3 & 172.3 & 322.9 & 314.8 \\
$1.5-2.0$ & 141.4 & 141.4 & 248.6 & 248.6 \\
$2.0-2.5$ & 80.9 & 51.7 & 158.0 & 99.1 \\
$2.5-3.0$ & 56.6 & 48.5 & 129.8 & 129.8 \\
$3.0-3.5$ & 46.9 & 46.9 & 90.4 & 90.4 \\
$3.5-4.0$ & 25.8 & 23.4 & 49.0 & 38.1 \\
$4.0-4.5$ & 13.6 & 13.6 & 33.2 & 33.2 \\
$4.5-5.0$ & 7.5 & 7.5 & 15.8 & 15.8 \\
\hline Total & 182.9 & 170.9 & 363.2 & 363.2 \\
\hline
\end{tabular}

The limits shown here are for the specific mass value of $1540 \mathrm{MeV} / \mathrm{c}^{2}$, similar limits can be obtained for nearby masses. The units in this table are $\mathrm{fb}$ per $\mathrm{GeV} / c$, and apply to the sum of all possible production processes. To isolate continuum and $\Upsilon(4 S)$ production, the values have been divided by the respective cross sections, and the corresponding limits on the multiplicities per event are given in table 3.4. 
Table 3.4: Upper limits on the multiplicity of $\Theta_{5}^{+}$per continuum and $\Upsilon(4 S)$ event. The units are $10^{-5}$ particles per event per $\mathrm{GeV} / c$ for the differential limits, and $10^{-5}$ particles/event for the total limits.

\begin{tabular}{|c|rr|rr||rr|rr|}
\hline & \multicolumn{4}{|c||}{ Continuum } & \multicolumn{4}{c|}{$\Upsilon(4 S)$} \\
p* Range & $\Gamma=1$ & $\mathrm{MeV}$ & \multicolumn{2}{|c||}{$\Gamma=8$} & $\mathrm{MeV}$ & $\Gamma=1$ & $\mathrm{MeV}$ & $\Gamma=8 \mathrm{MeV}$ \\
$\mathrm{GeV} / c$ & $\mathrm{~A}$ & $\mathrm{~B}$ & $\mathrm{~A}$ & $\mathrm{~B}$ & $\mathrm{~A}$ & $\mathrm{~B}$ & $\mathrm{~A}$ & $\mathrm{~B}$ \\
\hline $0.0-0.5$ & 6.36 & 6.36 & 14.04 & 14.04 & 22.7 & 22.7 & 50.2 & 50.2 \\
$0.5-1.0$ & 6.80 & 5.31 & 13.09 & 11.36 & 24.3 & 19.0 & 46.8 & 40.6 \\
$1.0-1.5$ & 5.08 & 5.08 & 9.53 & 9.29 & 18.2 & 18.2 & 34.0 & 33.2 \\
$1.5-2.0$ & 4.17 & 4.17 & 7.33 & 7.33 & 14.9 & 14.9 & 26.2 & 26.2 \\
$2.0-2.5$ & 2.39 & 1.52 & 4.66 & 2.92 & 8.5 & 5.4 & 16.6 & 10.4 \\
$2.5-3.0$ & 1.67 & 1.43 & 3.83 & 3.83 & - & - & - & - \\
$3.0-3.5$ & 1.38 & 1.38 & 2.67 & 2.67 & - & - & - & - \\
$3.5-4.0$ & 0.76 & 0.69 & 1.45 & 1.12 & - & - & - & - \\
$4.0-4.5$ & 0.40 & 0.40 & 0.98 & 0.98 & - & - & - & - \\
$4.5-5.0$ & 0.22 & 0.22 & 0.47 & 0.47 & - & - & - & - \\
\hline Total & 5.39 & 5.04 & 10.71 & 10.71 & 18.9 & 17.8 & 36.9 & 36.6 \\
\hline
\end{tabular}

In order to quote limits on the total production cross section either the momentum spectrum must be known or believed not to vary rapidly on the scale of the bin size. The latter assumption is borne out by the MC studies described in the previous sections, therefore conservative, model independent upper limits are assigned to the total multiplicities in continuum $(\Upsilon(4 S))$ events by simply summing each differential cross section over the relevant $p^{*}$ range from zero to $5.3(2.5) \mathrm{GeV} / c$, taking into account the fact that most of the systematic error is common to all bins. The limits derived from these sums by the same method are listed as "Total" in tables 3.3 and 3.4.

Any postulated spectrum can be folded into our differential limit to obtain a limit on the total cross section assuming that spectrum. The integral (which corresponds to a flat distribution) is the worst case, and hence a conservative, model-independent limit. It should be noted that the limits on $\Theta_{5}^{+}$production are in the interesting region (see fig. 3.1): four times the worst case gives a limit of $1.1 \times 10^{-4} \Theta_{5}^{+}$per $e^{+} e^{-} \rightarrow q \bar{q}$ event; this can be compared with the "expected" value of $8 \times 10^{-4}$. To place this limit into context it is plotted against the expected rates for other known particles in fig. 3.24. It can be seen that under these assumptions, the rate for pentaquark production is much lower than that expected for "normal" baryons at this mass. 


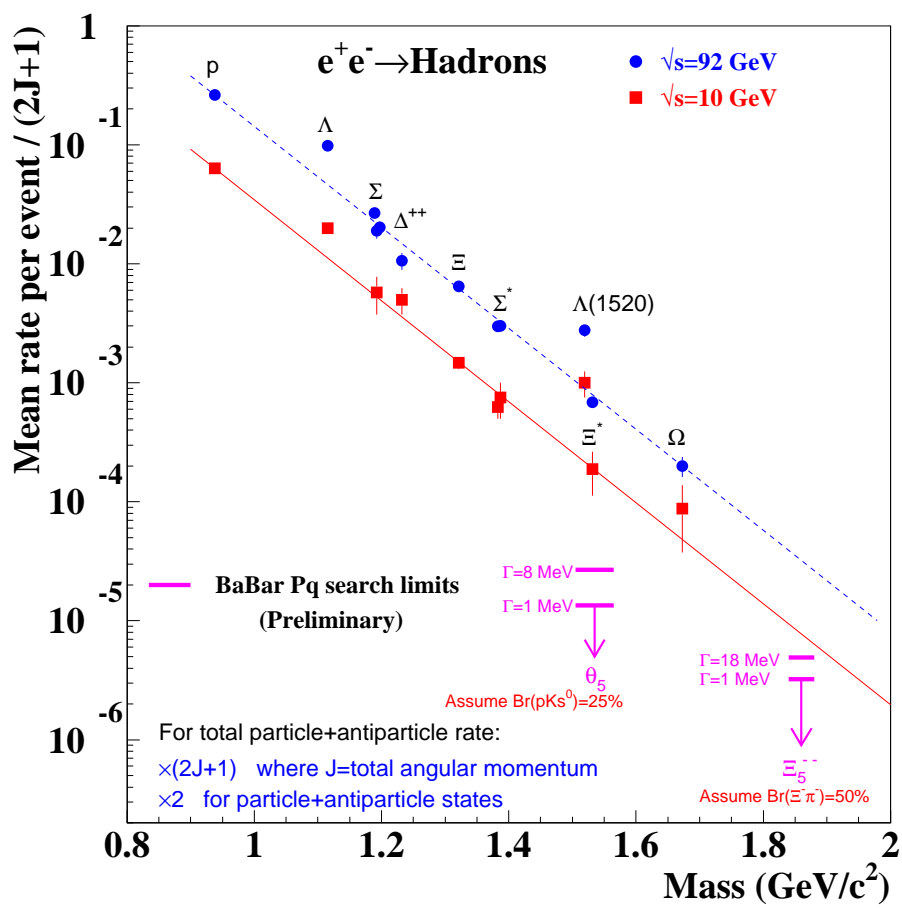

Figure 3.24: Compilation of light (no $c$ or $b$ quarks) baryon production rates in $e^{+} e^{-} \rightarrow$ hadrons from the PDG. Shown are the limits from BABAR on pentaquark production in $e^{+} e^{-} \rightarrow$ hadrons, at least an order of magnitude below the expected trend for "normal" baryons. 


\section{Chapter 4}

\section{Search for $\Theta(1540)^{+} \rightarrow K_{S}^{0} p$ in Electro- and Hadro-production}

\subsection{Motivation}

The results from experiments reporting the observation of the $\Theta_{5}(1540)^{+}$in a $K^{+} n$ or $K_{S}^{0} p$ invariant mass distribution are summarized in Table 1.1 of Chapter 1. As noted, seven of these experiments involve real or virtual photoproduction reactions, and four more involve hadroproduction on a hydrogen or nuclear target. Previously limits have been set on $\Theta_{5}^{+}$ production in $e^{+} e^{-}$annihilation. It follows that it would be of interest to extend the $B A B A R$ search to encompass electro- and hadro-production processes if it were possible to do so.

$B A B A R$ is in a unique position in terms of $e^{+} e^{-}$colliders, in that the design of the interaction region (IR), with the specific goal of high luminosity, has some interesting consequences. The Bending/Steering permanent magnets that bring the beams into collision in the luminous region cause off-orbit beam particles (beam-halo) to collide with the wall of the beam pipe, primarily in the horizontal plane. Although this effect is small compared to the primary $e^{+} e^{-}$interaction rate, the corresponding data sample when integrated over the entire Run 1-4 data taking period proves to be of considerable size ( $>10^{5}$ events). This then provides the opportunity to study quasi-real photo-production in beryllium of the $p K_{S}^{0}$ system, and hence to search for pentaquark production associated with such interactions.

With this motivation, a more general search for $p K_{S}^{0}$ production was carried out, in which the material of the entire inner $B A B A R$ detector was considered as a source of such events. These interactions are then generated not only by beam-halo electrons and positrons, but also potentially by background particles from all sources, and in addition as a result of the secondary interactions of hadrons produced in the primary $e^{+} e^{-}$collisions.

The objective of this study is to search for the existence of the $\Theta(1540)^{+}$. The MC resolution studies of the previous chapter indicated that the mass resolution for the $p K_{S}^{0}$ system is $\sim 2 \mathrm{MeV} / c^{2}$ (HWHM) independently of $p^{*}$, and it follows that the mass resolution 
in the material interactions studies should be similar, since it depends mainly on the proximity of the $\Theta^{+}$mass region to $p K_{S}^{0}$ threshold (i.e. the low Q-value for the decay mode involved). This means that any significant production of a narrow $(\Gamma \leq 1 \mathrm{MeV}) \Theta^{+}$ in the material interaction process should be observable; for example the $\Lambda(1520)(\Gamma \sim 15$ $\mathrm{MeV}$ ) is clearly observed in the course of this study.

No matter whether the $\Theta^{+}$is, or is not, observed there can be no meaningful MC study leading to associated cross section estimates or upper limits. In general, the composition of the detector background particle sample is unknown, as is its variation over the Run 1-4 time period. Even for the beam-halo, electron and positron contribution the considerable time variation illustrated in Table 3 of Appendix B, is not known in any detail. Even if the composition and momentum spectrum were known in detail, any useful Monte Carlo would have to incorporate detailed information on inclusive production of the $K_{S}^{0} p$ system (including angular distributions as a function of $p K_{S}^{0}$ mass) for each of the particle types contributing to background interacting with $\mathrm{Be}, \mathrm{Ni}, \mathrm{Au}, \mathrm{H}_{2} \mathrm{O}, \mathrm{Fe}, \mathrm{Si}, \mathrm{C}$ etc., etc. Such information does not exist and so it follows that such a restriction applies even to Monte Carlo simulation of material interactions due to the secondary interactions of hadrons resulting from the primary $e^{+} e^{-}$collisions. In addition, the $\mathrm{MC}$ describing the detector geometry and structure does not include material outside of the tracking volume. The aim of the material interactions study is simply to search for the evidence of $\Theta^{+}$production in a context beyond that $e^{+} e^{-}$interactions, one which is more akin to many of the processes in which it is claimed that $\Theta^{+}$production has been observed.

In the $B A B A R$ experiment, a previous study of interactions in the detector material yielding an identified proton has been carried out with the aim of checking the radial and longitudinal length scales of the BABAR coordinate system [77]. The distribution of the resulting proton-associated vertices is shown in $y-x$ projection in fig. 4.1. The beampipe, SVT, r.f. shield, and the two innermost SVT layers are seen quite clearly. Of particular interest are the two high-intensity regions (denoted by "Hot Spot") concentrated in the beampipe region in the horizontal plane. The fact that these hot spots are localized to the plane in which the $e^{+}$and $e^{-}$beams are bent into head-on collision by the B1/Q1 vertical dipole magnets suggests very strongly that they result primarily from beam halo interactions in the Be beampipe. This is demonstrated schematically in the plan view of the interaction region shown in fig. 4.2. Here it is indicated that off-beam electrons would tend to be bent into the beampipe at $x<0$, while off-beam positrons would be bent into collision at $x>0$. The hot spot at negative $x$ is much more intense than that at positive $x$, and so provides clear evidence for significant proton production as a result of the interaction of $\sim 9 \mathrm{GeV}$ electrons with the Be beampipe.

In the course of a study of inclusive production of $K_{S}^{0}$ mesons in $e^{+} e^{-}$interactions, the point of closest approach (POCA) of the flight-path of each $K_{S}^{0}$ candidate and the $e^{+} e^{-}$ collision axis was obtained. The $x-y$ scatterplot for this point relative to the collision axis is shown in fig. 4.3 for the $K_{S}^{0}$ sample. The high intensity region around the collision 


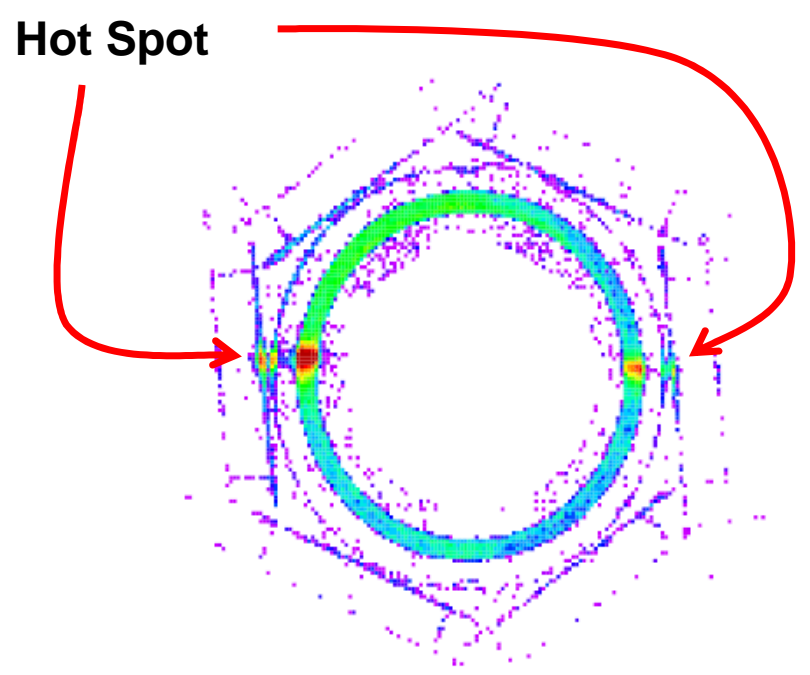

Figure 4.1: The $y$ vs. $x$ scatterplot for the proton-associated vertex locations from [77], using $\sim 2 \mathrm{fb}^{-1}$ of $e^{+} e^{-}$luminosity.

axis, $\mathrm{R}<0.3 \mathrm{~cm}$ and $\left|z-z_{I P}\right|<3.2 \mathrm{~cm}$, has been excluded in order to reveal the structure in the rest of the plot. Two approximately circular ${ }^{1}$ loci, each of radius $\sim 1.25 \mathrm{~cm}$, are observed, the one with center at negative $x$ being much more intense than the other. The accompanying sketch illustrates the interpretation of the circle with $x<0$. The POCA was calculated assuming that the $K_{S}^{0}$ originated from the collision axis (IP); the dashed line corresponds to the distance of closest approach (DOCA), and so is perpendicular to the $K_{S}^{0}$ flight-path. If, however, the point $\mathrm{S}$ is the actual source of the $K_{S}^{0}$ mesons, the geometry of the situation is such that the POCA in the $x-y$ projection describes a circle for which the line connecting $\mathrm{S}$ to IP is a diameter. [The angle in a semi-circle is a right-angle.] The interpretation of the circular loci in fig. 4.3 is then that the relevant $K_{S}^{0}$ mesons originate from points in the horizontal plane at $\sim \pm 2.5 \mathrm{~cm}$ from the IP i.e. from the beampipe hot spots of fig. 4.1, with the source at negative $x$ again being much more intense than that at positive $x$.

It follows from the above that we have clear evidence for the electroproduction of protons and $K_{S}^{0}$ mesons from the Be beampipe of the BABAR detector, and hence, given the evidence summarized in Table 1.1, it is of interest to investigate such interactions with a view to observing the $\Theta_{5}(1540)^{+}$via its $K_{S}^{0} p$ decay mode.

\subsection{Overview of the Analysis and Summary of Results}

Initially, the structure of the beampipe and of the inner detector in radius out to and including the inner cylinder of the Drift Chamber is reviewed. The analysis of the data commences by applying simple geometrical and invariant mass criteria to carry out a rather loose selection of $K_{S}^{0}$ candidates. For each $K_{S}^{0}$ candidate in a given event, the

\footnotetext{
${ }^{1}$ Note that the $x$ and $y$ scales in fig. 4.3 are different.
} 
DOCA for each remaining positively-charged track in that event to the flight-path of the $K_{S}^{0}$ candidate is calculated. If this is less than $1 \mathrm{~cm}$, the $d E / d x$ information from the SVT and/or DCH for this track is examined in order to ascertain that it is consistent within loose requirements with being a proton. The mid-point of the line corresponding to the DOCA is defined as the approximate position of the candidate $\left(K_{S}^{0}, p\right)$ production vertex, and the radius of this point in the $(x, y)$ plane is required to be $>2 \mathrm{~cm}$. Events for which at least one such candidate is found are retained for further study. In this way only events which originate from $e^{+} e^{-}$collisions are excluded, and events resulting from interactions in any part of the detector material are retained, whether they be due to electroproduction or the secondary interactions of hadrons produced in primary $e^{+} e^{-}$collisions.

This all-inclusive approach is taken since the primary objective of the analysis is to observe a $\Theta_{5}(1540)^{+}$signal and to obtain precise measurements of its mass and, if possible, its width, regardless of how it is being produced. The analysis can be restricted subsequently to candidates resulting from electroproduction in the Be beampipe in order to make contact with the relevant analyses summarized in Table 1.1.

In the second phase of the analysis, the geometrical selection criteria are significantly tightened, and, after detailed study of the available $d E / d x$ information, stringent consistency requirements are made of the proton candidate, and also of the $\pi^{+}$and $\pi^{-}$tracks forming the $K_{S}^{0}$ candidate. At this stage, it is found that the spatial distribution of the surviving $\left(K_{S}^{0}, p\right)$ vertices reproduces to high accuracy the known geometry of the beampipe and inner $B A B A R$ detector. The background-subtracted $K_{S}^{0}$ proper time distribution with respect to these vertices also exhibits reasonable behavior. This establishes confidence that the surviving $\left(K_{S}^{0}, p\right)$ candidates do, for the most part, correspond to hadron or electron interactions in the detector material. The lab. momentum distributions of the $K_{S}^{0}$ and proton are almost entirely confined to the region below $1.5 \mathrm{GeV} / c$ and are thus

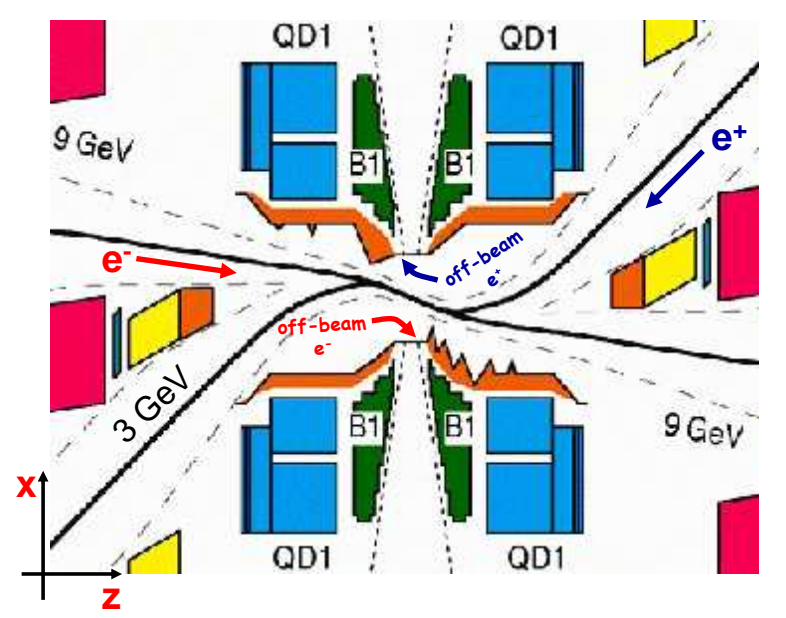

Figure 4.2: A schematic plan view of the interaction region. The expected bending directions of off-beam electrons and positrons are indicated. 


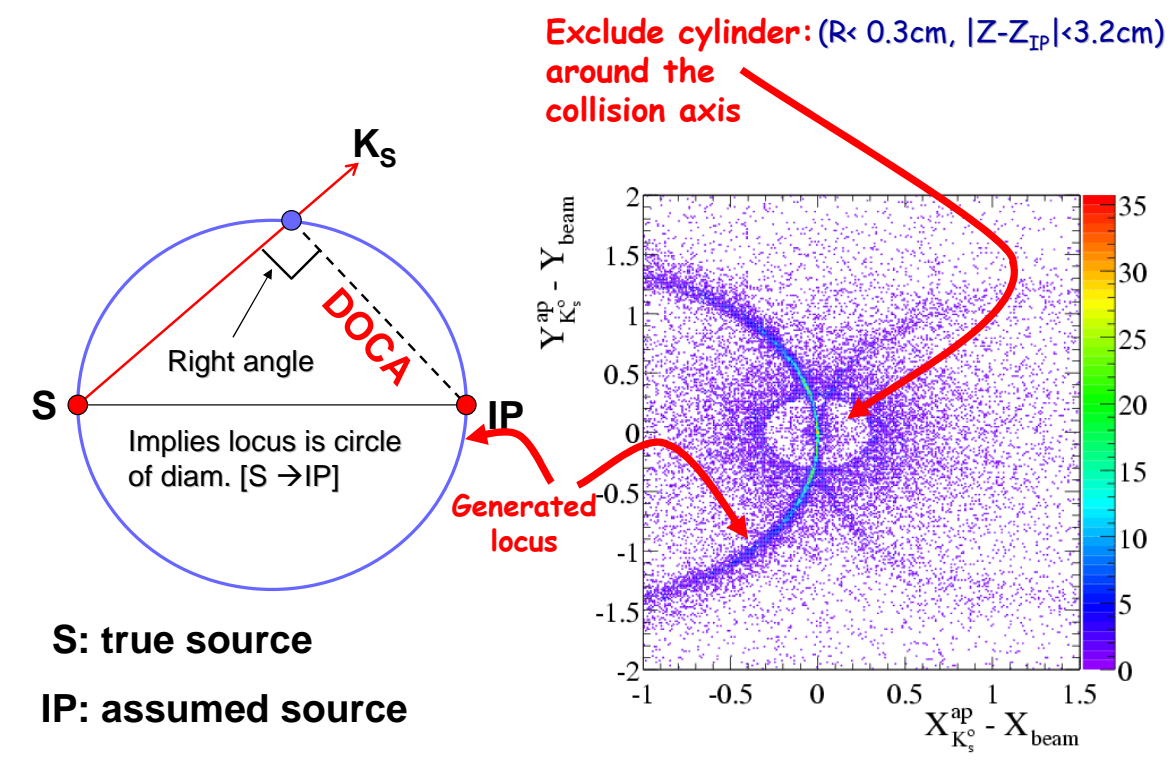

Figure 4.3: Evidence for $K_{S}^{0}$ production from the beampipe wall; the interpretation of the scatterplot and sketch is given in the text.

very similar to those observed in many of the experiments listed in Table 1.1. However the high statistics $K_{S}^{0} p$ invariant mass distribution obtained at this point in the analysis shows no evidence of a $\Theta_{5}(1540)^{+}$signal.

For each $\left(K_{S}^{0}, p\right)$ vertex, the POCA for all other charged tracks in the same event is obtained, and those tracks having DOCA $<5 \mathrm{~mm}$ are flagged as being associated with this vertex. Approximately two-thirds of all $\left(K_{S}^{0}, p\right)$ vertices have at least one associated track. The $K_{S}^{0} p$ invariant mass distribution for these candidates shows no $\Theta_{5}(1540)^{+}$ signal. Using $d E / d x$ and DIRC information, sub-samples of charged tracks corresponding to baryons, $\pi^{ \pm}, K^{ \pm}$and $e^{ \pm}$can be identified. The presence of an associated baryon indicates that the reaction yielding the $\left(K_{S}^{0}, p\right)$ candidate involves nuclear break-up, and hence is complex. The $K_{S}^{0} p$ mass distribution remaining after removing such events still shows no structure.

After removing events with an associated baryon, surviving events with a $\pi^{+}, \pi^{-}, K^{-}$ or $K^{+}$are examined. A clear $K^{*}(892)$ signal is observed in the $K_{S}^{0} \pi^{+}$mass distribution, while there is a much smaller $\bar{K}^{*}(892)$ signal in $K_{S}^{0} \pi^{-}$. This suggests that the majority of the $K_{S}^{0}$ candidates are in fact $K^{0}$ 's rather than $\bar{K}^{0}$ 's, and that they are primarily produced in reactions involving strange baryons or hyperon resonances. There is direct evidence of this from the corresponding $p \pi^{-}$mass distribution, which shows a strong $\Lambda$ signal. The $K_{S}^{0} K^{-}$and $K_{S}^{0} K^{+}$mass distributions show evidence of $a_{0}(980)$ production, and a clear $\Lambda(1520)$ signal is present in the $p K^{-}$spectrum. The latter also shows evidence of $\Lambda(1405)$ production. The $K_{S}^{0} p$ mass distribution is examined for each of the above sub-categories, but no $\Theta_{5}(1540)^{+}$signal emerges.

For events with an associated $K^{-}$, the $K^{-} K_{S}^{0} p$ mass distribution is confined to the low mass region. This is similar to the $K^{-} K^{+} n$ mass distribution obtained for the CLAS 
reaction $\gamma p \rightarrow \pi^{+} K^{-} K^{+} n$ when low momentum-transfer-squared from the photon to the $\pi^{+}$was required [41]. CLAS reported a strong correlation between the $\Theta_{5}(1540)^{+}$signal and the $K^{-} K^{+} n$ mass region around $2.4 \mathrm{GeV} / c^{2}$, suggesting a possible $N^{*}$ decay to $K^{-}$ $\Theta_{5}^{+}$. When the $K^{-} K_{S}^{0} p$ mass region around $2.4 \mathrm{GeV} / c^{2}$ is selected for the BABAR data sample, no $\Theta_{5}(1540)^{+}$signal is observed.

All of these procedures are repeated after requiring that the $\left(K_{S}^{0}, p\right)$ vertex be localized to the region of the Be beampipe in the horizontal plane corresponding to electroproduction. Although the statistics are significantly reduced, no $\Theta_{5}(1540)^{+}$signal is observed. For a small subset of these events, the scattered electron is identified, but again there is no evidence of $\Theta_{5}(1540)^{+}$production.

A comparison is made between the electroproduction results obtained in the BABAR analysis and those obtained from the HERMES[49] and ZEUS[50] experiments in which observation of the $\Theta_{5}(1540)^{+}$signal is claimed (see Table 1.1). Finally, the BABAR data on the electroproduction of the $K_{S}^{0} p K^{-}$system are compared to the SAPHIR data [42] on the photoproduction of the $K^{+} n \bar{K}^{0}$ final state.

\subsection{The Beampipe and Inner Detector Region}

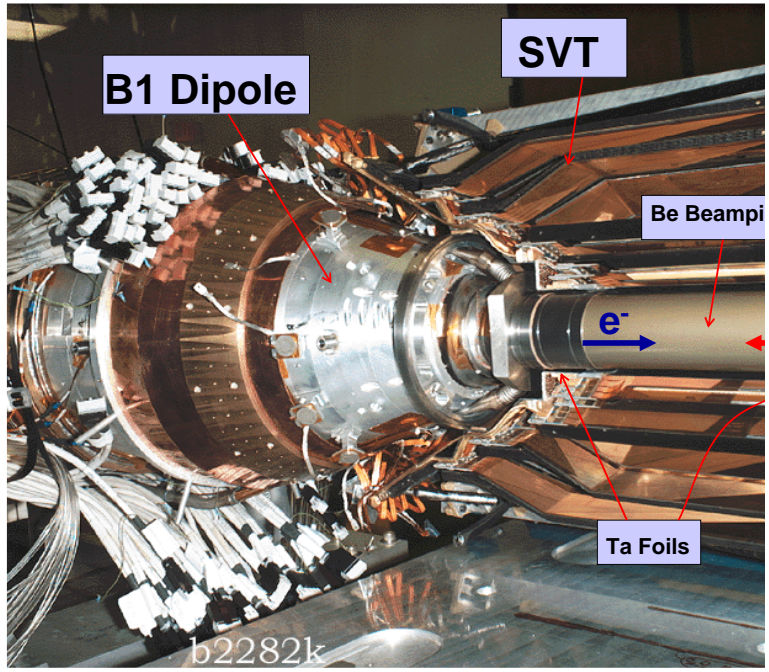

Figure 4.4: The beampipe, backward B1 magnet and positive $x$ half of the SVT.

\section{The Beam-Pipe Radial Geometry}

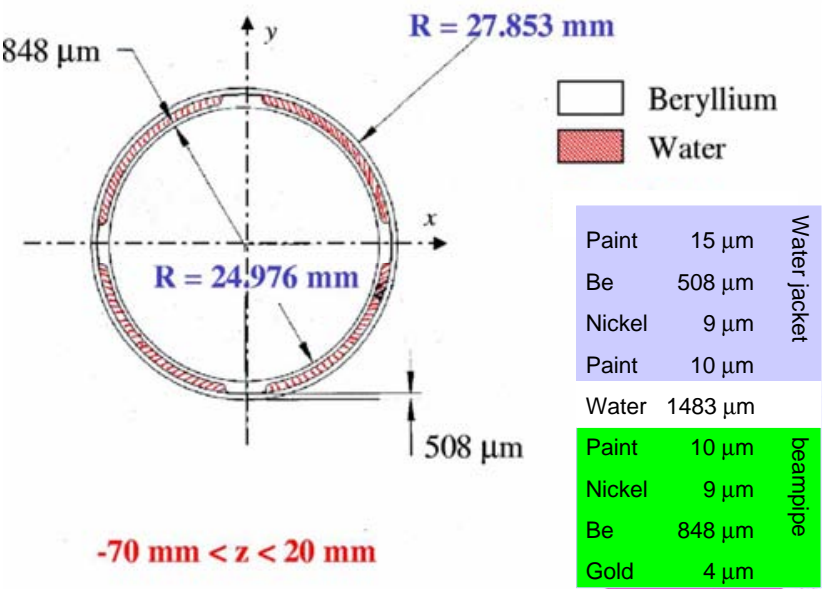

Figure 4.5: The radial geometry and material composition of the beampipe.

The beampipe, and the detector components immediately surrounding it, provide the target material for the present study. It follows that the extent to which the reconstructed $\left(K_{S}^{0}, p\right)$ vertices reproduce the geometrical structures in this region provides a stringent test of the quality of the analysis data sample. Consequently, by way of introduction, a brief description of the detector elements involved is presented.

Fig. 4.4 shows the Be beampipe and the backward $(z<0)$ B1 magnet in the process of being captured by the positive $x$ half of the SVT; the five-layer structure of the SVT can be seen, and the directions of the colliding beams are indicated for the purposes of orientation. 


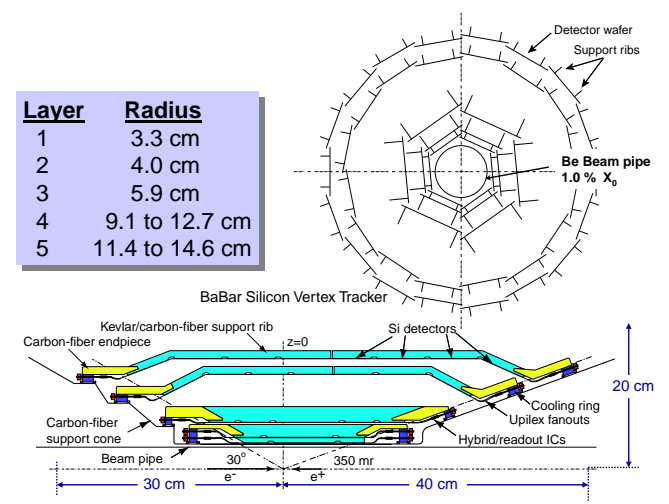

Figure 4.6: The geometry of the SVT layers.

The radial structure and composition of the beampipe are illustrated in fig. 4.5. The inner and outer radii are $\sim 2.5 \mathrm{~cm}$ and $\sim 2.8 \mathrm{~cm}$ respectively, and it should be noted that in the hot spot regions of fig. 4.1, the beampipe consists almost entirely of Be. The forward and backward regions of the beampipe outside the tracking volume are wrapped in Ta foil in order to reduce X-ray background in the SVT and drift chamber (fig. 4.4). This high-Z material significantly enhances the interaction rate of off-beam electrons and positrons w.r.t. that observed in the Be beampipe, as will be shown later. Between the beampipe and the first layer of the SVT, there is a cylindrical r.f.shield consisting of 100 microns of carbon fiber coated with 20-micron aluminum foil.

The layer structure of the SVT is shown schematically in fig. 4.6. The three innermost layers are hexagonal in cross-section, while layers 4 and 5 consist of 16- and 18-sided polygons respectively, and have lampshade structures fore and aft. Every Si wafer is supported on the outside by two Kevlar-carbon fiber ribs.

The completed SVT mounted between the B1/Q1 magnets is shown in fig. 4.7(a), where the carbon fiber tubes forming the SVT support cage are also evident. In fig. 4.7(b), the SVT region has been enclosed in an aluminized-kapton gas bag, which also serves as the outer SVT r.f. shield. The entire assembly is then installed inside the Support Tube (fig. 4.7(c)), which consists of a central section of $2 \mathrm{~mm}$ thick carbon fiber mounted between two half-inch thick stainless steel cylinders. The drawing of fig. 4.7(d) shows how the Support Tube is positioned w.r.t. the rest of the BABAR detector such that the carbon fiber section is aligned with the tracking volume.

In this location, the outer radius of the Support Tube is within $\sim 2 \mathrm{~cm}$ of the inner cylinder of the Drift Chamber. The central section of this cylinder is made of $1 \mathrm{~mm}$ thick Be coated with anti-corrosion paint (BR127), while the fore and aft sections consist of $5 \mathrm{~mm}$ thick aluminum. In fig. 4.8 this cylinder is shown during chamber stringing at TRIUMF; the green hue of the Be section is due to the BR127 paint.

The inner cylinder of the Drift Chamber defines the outer limit of the detector material within which interaction vertices can be reconstructed in significant numbers, although some vertices can actually be found in the upstream endplate, as can be seen in fig. 4.23. 

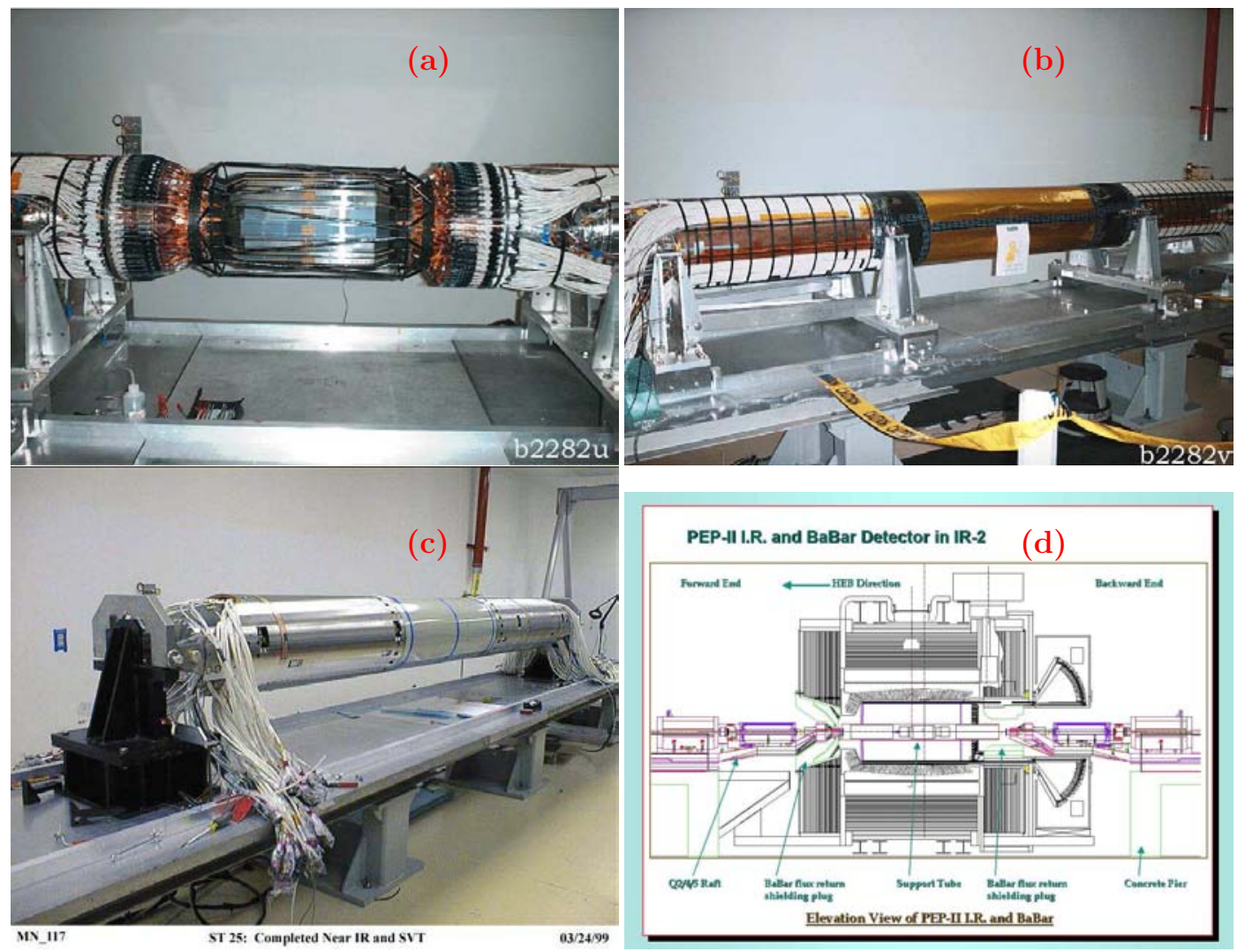

Figure 4.7: (a) The SVT mounted between the B1 magnets, (b) the completed B1/Q1/SVT complex, (c) the completed support tube and contents, (d) schematic of the PEP II IR and the BABAR detector indicating the location of the Support Tube after installation.

\subsection{Initial Selection of Candidate Events}

Initially the search is performed with loose, efficient selection criteria similar to those used for the $\Theta_{5}(1540)^{+}$inclusive analysis of $e^{+} e^{-}$annihilation data. In the present analysis the ultimate interest is in electron and positron interactions in the material of the beampipe.

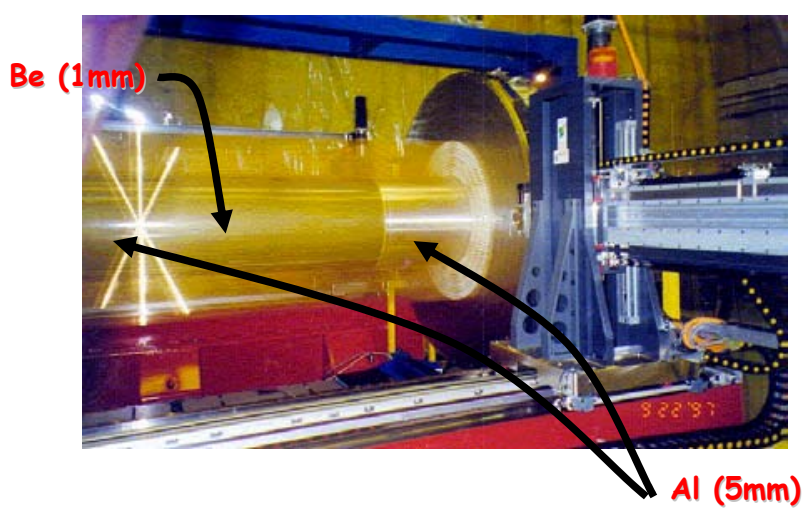

Figure 4.8: The DCH inner cylinder during stringing of the Drift Chamber at TRIUMF. 
However the study is not restricted only to the electroproduction regions, since there is a large gain in statistics to be obtained by considering interactions in all of the detector material, whether they be due to electro- or hadro-production. The main difference from the original $e^{+} e^{-}$search is that the detector geometry is used to reject candidates produced from the IR instead of to select them. The search for the $\Theta_{5}(1540)^{+}$can be restricted readily to the electroproduction region of the beampipe at a later stage of the analysis.

Events are selected with at least one $K_{S}^{0} \mathrm{p}$ candidate satisfying the following criteria:

- $K_{S}^{0}: \operatorname{DOCA}\left(\pi^{+} \pi^{-}\right)<0.6 \mathrm{~cm},\left|m\left(\pi^{+} \pi^{-}\right)-m(P D G)\right| \leq 50 \mathrm{MeV} / c^{2}$

The distribution of $\operatorname{DOCA}\left(\pi^{+} \pi^{-}\right)$values is shown in fig. $4.9(\mathrm{a})$; the $0.6 \mathrm{~cm}$ requirement is clearly very conservative, and there is no discernible $K_{S}^{0}$ signal for the rejected vertices.
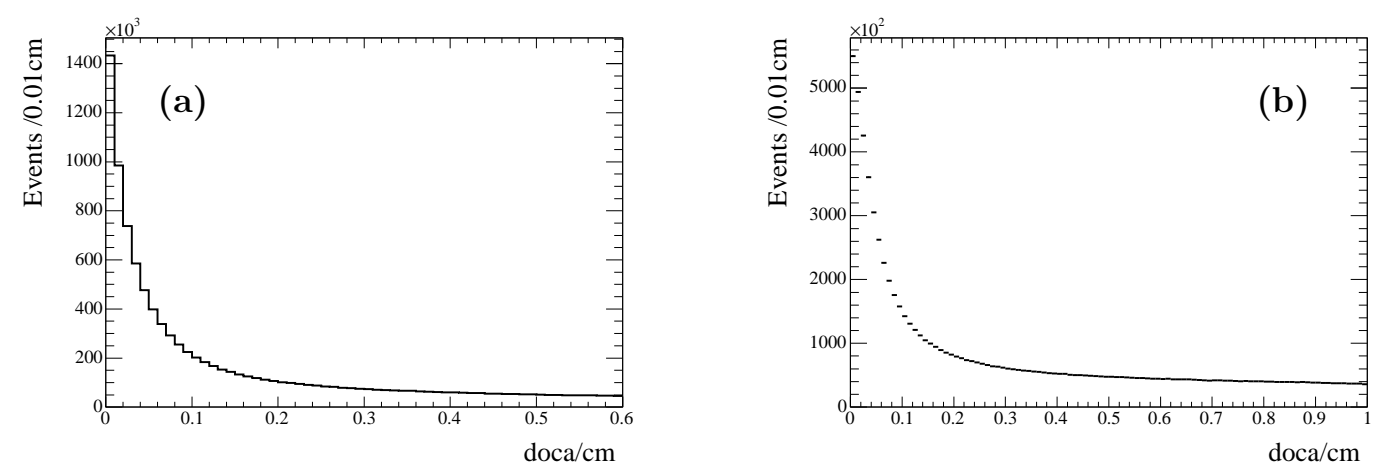

Figure 4.9: (a) DOCA $\left(\pi^{+} \pi^{-}\right)$for the candidate $K_{S}^{0}$ vertices retained by the requirement DOCA $<0.6 \mathrm{~cm}$; (b) DOCA between the $K_{S}^{0}$ candidate flight-path and the proton candidate track for candidates retained by requiring $\mathrm{DOCA}<1 \mathrm{~cm}$

- DOCA between $p$ candidate and $K_{S}^{0}$ flight-path $<1 \mathrm{~cm}$

The distribution in the DOCA between the $K_{S}^{0}$ flight-path and the proton candidate is shown in fig. 4.9(b); clearly the requirement $\mathrm{DOCA}<1.0 \mathrm{~cm}$ is very conservative. The $\left(K_{S}^{0}, p\right)$ candidate vertex position is chosen to be the mid-point of the DOCA.

- $\mathrm{R}\left(K_{S}^{0}, p\right)$ vertex in the $x-y$ plane $>2 \mathrm{~cm}$ from the $z$-axis; no other geometrical restriction

The inner radius of the beampipe is $\sim 2.5 \mathrm{~cm}$ (see fig. 4.5) and so there is no loss of candidate vertices within the detector material as a result of this requirement.

- Signed Flight Length (SFL) from $\left(p K_{S}^{0}\right)$ vertex to $K_{S}^{0}$ vertex $>0.1 \mathrm{~cm}$

This greatly reduces combinatoric background with no significant loss of $K_{S}^{0}$ signal.

- Very loose proton PID; no PID on the $\pi^{-}$or $\pi^{+}$from $K_{S}^{0}$ decay

The proton identification procedure is discussed in detail in the next sub-section. 


\subsubsection{Proton Identification}

The candidate proton tracks from the $\left(K_{S}^{0}, p\right)$ vertex sample are divided into three classes depending on the nature of the coordinates on the track as follows:

$\begin{array}{lll}\text { Class 1: } & \text { SVT only } & (\sim 6 \%) \\ \text { Class 2: } & \text { SVT \& DCH } & (\sim 85 \%) \\ \text { Class 3: } & \text { DCH only } & (\sim 9 \%)\end{array}$

with the fractional populations indicated. Almost all tracks $(\sim 94 \%)$ have DCH information, and $\sim 91 \%$ have SVT information. Measured specific energy loss $(d E / d x)$ is shown as a function of lab. momentum in fig. 4.10(a) for the SVT and in fig. 4.10(b) for the DCH. Bands characteristic of $\pi^{+}, K^{+}, p$, deuteron $(d)$ and triton $(t)$ are evident in fig. 4.10 (b), and there is even a faint band consistent with ${ }^{4} \mathrm{He}^{++}$; resolution is $\sim 8 \%$ of $d E / d x$. Corresponding bands are present in fig. 4.10(a), but the resolution is only $\sim 17 \%$ of $d E / d x$ (typically only 6 SVT samples are used per track) and so no $K^{+}$band can be seen.
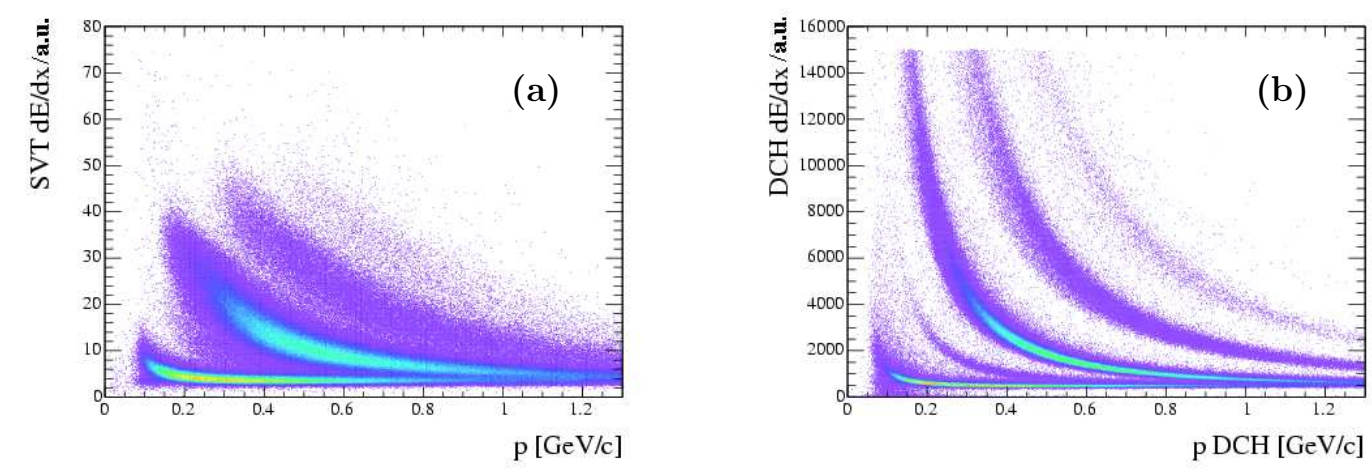

Figure 4.10: (a) The distribution of $d E / d x$ measured in the SVT versus lab. momentum for candidate proton tracks forming a vertex with a candidate $K_{S}^{0} ; \pi^{+}, p, d$ and $t$ bands are evident; (b) the corresponding plot for $d E / d x$ measured in the $\mathrm{DCH}$; as a result of the better resolution, a $K^{+}$band is seen in addition to those present in (a); $p$ DCH is the track momentum at the entrance to the $\mathrm{DCH}$.

In fig. 4.10, the lab. momentum distribution for tracks in the proton band is concentrated in the region below $\sim 1.2 \mathrm{GeV} / c$, and peaks at $\sim 0.5 \mathrm{GeV} / c$; for $\pi^{+}$, the distribution is mainly below $0.9 \mathrm{GeV} / c$, peaks at $\sim 0.3 \mathrm{GeV} / c$ and is well-separated from the proton band. It follows that proton identification can be performed with no need to resort to the DIRC. In order to do this, a proton consistency, $C(p)$, is defined by:

$$
C(p)=\frac{\frac{d E}{d x}^{\text {meas }}-{\frac{d E^{c a l c}}{d x}}^{c}(p)}{{\frac{d E^{c a l c}}{d x}}^{\text {( }}(p)}
$$

and it is required that $C(p)$ be within a specified tolerance of 0 ; here $\frac{d E}{d x}^{c a l c}(p)$ is the Bethe-Bloch function for the relevant run period evaluated using the proton mass. 
The scatterplots of $C(p)$ versus lab. momentum corresponding to fig. 4.10(a) and fig. 4.10(b) are shown in fig. 4.11(a) and fig. 4.11(b) respectively. The regions within which candidate protons are retained are defined as follows:
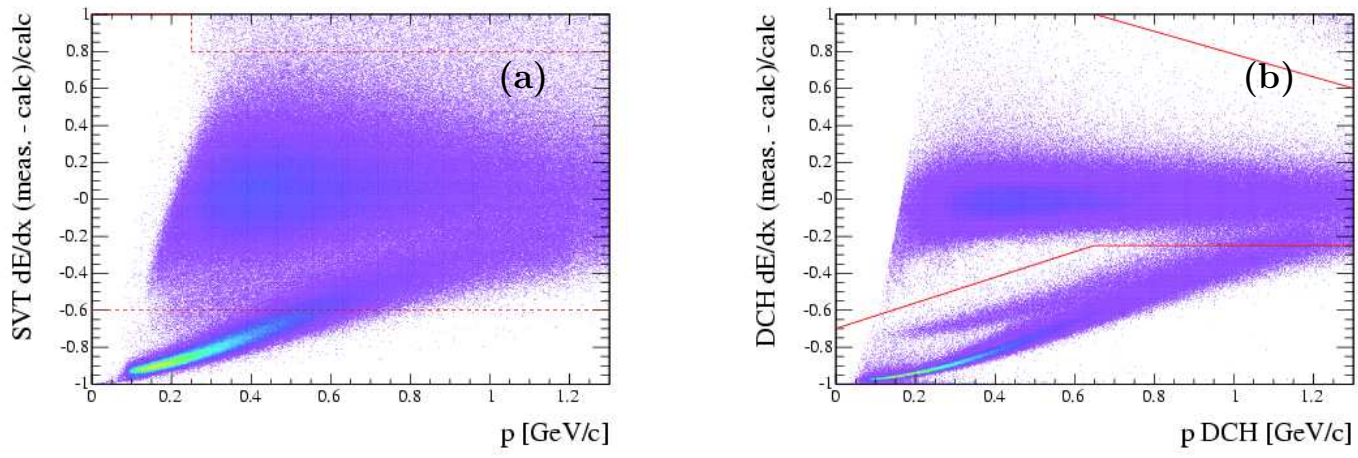

Figure 4.11: (a) The distribution of $C(p)$ in the SVT versus lab. momentum corresponding to fig. 4.10(a); (b) the distribution of $C(p)$ in the DCH, versus momentum at the DCH entrance corresponding to fig. 4.10(b). The red lines in both figures denote regions within which candidate protons are retained.

(a) For tracks with only SVT coords. (i.e. class 1):

$$
\begin{aligned}
C(p) & \geq-0.6 & & \\
\text { and } C(p) & \leq 1.0 & & (p \leq 0.250 \mathrm{GeV} / c), \\
C(p) & \leq 0.8 & & (p \geq 0.250 \mathrm{GeV} / c) .
\end{aligned}
$$

(b) For tracks with DCH coords. (i.e. classes 2 and 3):

$$
\begin{array}{rr}
C(p) \geq-0.70+0.45(p / 0.65) & (p \leq 0.650 \mathrm{GeV} / c) \\
C(p) \geq-0.25 & (p \geq 0.650 \mathrm{GeV} / c) \\
\text { and } & \\
C(p) \leq 1.4-1.1(p / 1.8) & (p \leq 1.8 \mathrm{GeV} / c) \\
C(p) \leq 0.3 & (p \geq 1.8 \mathrm{GeV} / c) .
\end{array}
$$

Clearly, these criteria are very conservative, and no attempt is made at this point to remove residual pion and kaon contamination.

After initial selection of events containing at least one $K_{S}^{0} p$ candidate has been completed, the kinematic and geometric quantities characterizing all candidates from these 
events are investigated.

The distribution in the cosine of the helicity angle $\Theta_{H}$, of the $\pi^{+}$from the candidate $K_{S}^{0}$ is considered first; $\Theta_{H}$ is the angle between the $\pi^{+}$direction in the $K_{S}^{0}$ rest frame and the $K_{S}^{0}$ direction in the lab. frame. For the decay of real $K_{S}^{0}$ mesons the distribution is flat. This distribution is shown in fig. 4.12(a) for a sub-sample of the $\left(K_{S}^{0}, p\right)$ candidates selected thus far. Clearly, the distribution is not flat. The peaks near $\cos \Theta_{H}= \pm 1$ result from $e^{+} e^{-}$pairs for which the electrons have been misinterpreted as pions. The peak near $\cos \Theta_{H}=0.85$ is due to $\Lambda$ 's for which the proton has been misinterpreted as a $\pi^{+2}$, and the steep slope toward $\cos \Theta_{H} \sim 1$ is due to general misinterpretation of positively-charged baryons [protons, deuterons, and tritons; see below] as pions. The absence of a $\bar{\Lambda}$ peak, and of any slope toward $\cos \Theta_{H} \sim-1$ indicates that an event sample has been selected which is already rich in tracks associated with material interactions, since there is no indication of background resulting from anti-baryons being misinterpreted as $\pi^{-}$'s.

It is clear from fig.4.12(a), that stringent PID requirements must be applied to the pions forming the $K_{S}^{0}$ candidates. This is discussed below.
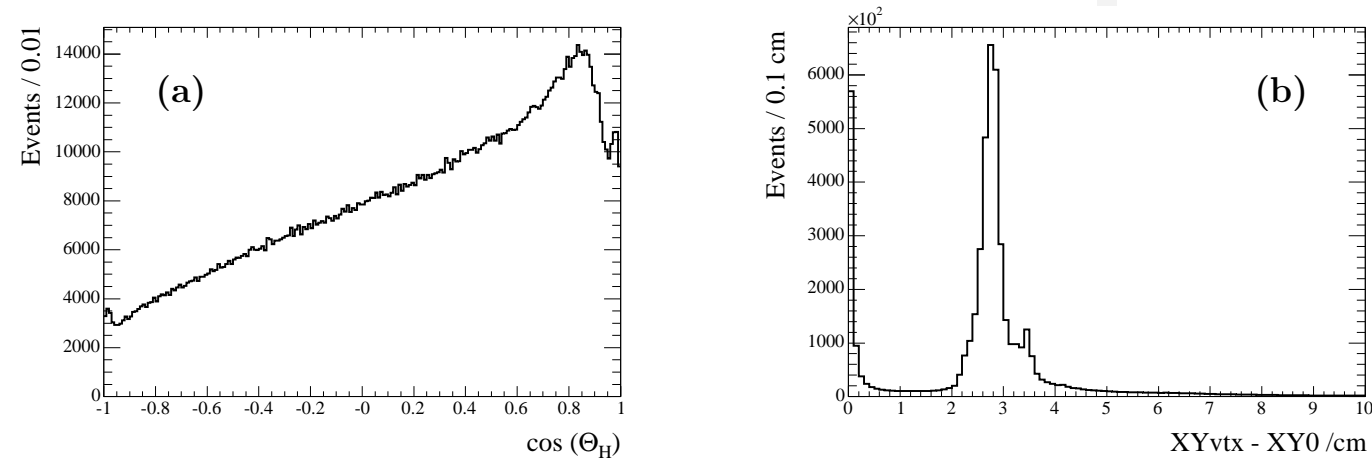

Figure 4.12: (a) Candidate $K_{S}^{0}$ distribution in $\cos \Theta_{H}$ (no PID has been applied); (b) candidate $K_{S}^{0}$ vertex distribution in the $(x, y)$ plane relative to the $e^{+} e^{-}$collision axis.

The distribution of the distance in the $(x, y)$ plane between the IP and a candidate $K_{S}^{0}$ vertex is shown in fig. 4.12(b). The peak near zero indicates that a significant number of $K_{S}^{0}$ candidates result from random combinations of charged particles from $e^{+} e^{-}$interactions. This peak is removed by requiring that the distance be greater than $0.3 \mathrm{~cm}$; this causes very little loss of real $K_{S}^{0}$ decays.

\subsubsection{Rejection of Misidentified Protons}

From fig. 4.11(a), it is clear that the SVT-only proton sample suffers from pion/kaon contamination for momenta greater than $\sim 0.4 \mathrm{GeV} / c$. A similar effect is apparent in fig. 4.11(b) for DCH identified protons for momenta greater than $\sim 1 \mathrm{GeV} / c$. In both instances, the pion and kaon bands have merged before overlap occurs with the proton

\footnotetext{
${ }^{2}$ A full explanation is given in: http://www.slac.stanford.edu/ wmd/bbkinematics/mass_helicity_mapping.note
} 
band, and so if the pions are removed essentially all of the kaons should be removed also. In order to remove the pions, a pion consistency, $C(\pi)$, is defined just as for $C(p)$, and plotted versus momentum for the candidate protons selected thus far. This is shown in fig. 4.13(a) for proton candidates with SVT information, and in fig. 4.13(b) for those with DCH information. In each plot there is a significant horizontal band centered at 0 which shows quite clearly the presence of pion/kaon contamination.
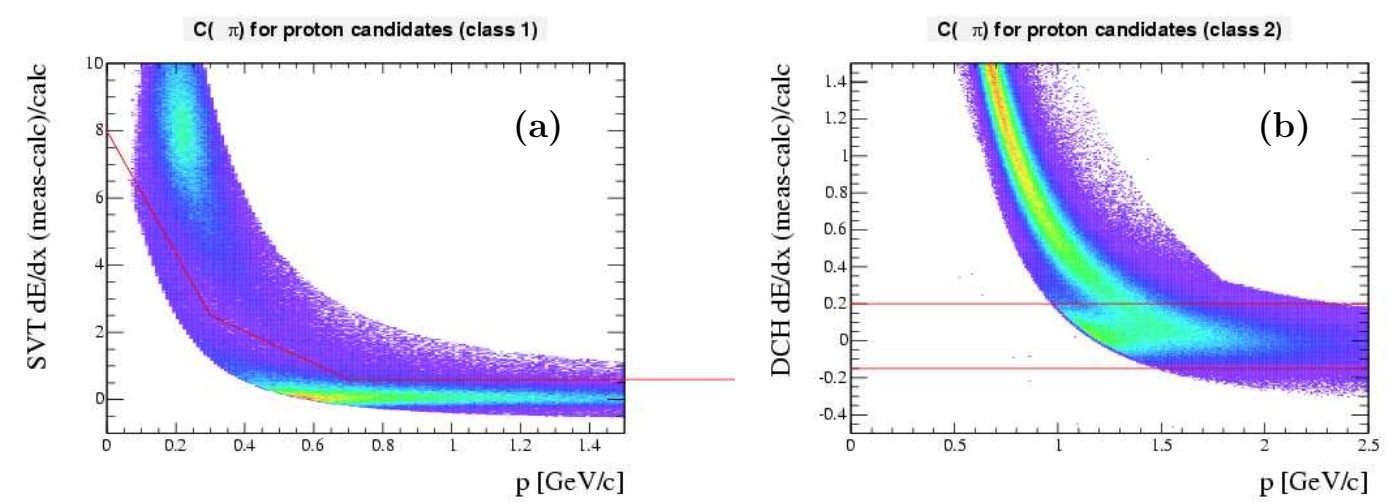

Figure 4.13: (a) The distribution of $C(\pi)$ in the SVT versus lab momentum for the selected proton candidates; SVT only tracks which fall below the red boundary are rejected; (b) the distribution of $C(\pi)$ in the DCH versus lab. momentum at the DCH entrance; proton candidates which fall inside the horizontal band at $C(\pi) \sim 0$ are rejected.

Tracks with $C(\pi)$ in the following regions are rejected as proton candidates:

(a) For tracks with only SVT coords (i.e. class 1):

$$
\begin{aligned}
C(\pi) \leq 8.0-5.5(p / 0.3) & (p \leq 0.3 \mathrm{GeV} / c) \\
C(\pi) \leq 2.5-1.9(p-0.3) / 0.4 & (0.3 \leq p \leq 0.7 \mathrm{GeV} / c) \\
C(\pi) \leq 0.6 & (p \geq 0.7 \mathrm{GeV} / c) .
\end{aligned}
$$

(b) for tracks with $\mathrm{DCH}$ coords (i.e. class 2 and 3 ):

$$
-0.15 \leq C(\pi) \leq 0.20
$$

For surviving tracks of class 2 a small number had SVT $d E / d x$ information which indicated that they were pions. These are interpreted as pions which interacted in the material outside the SVT to produce a forward going proton, which was then identified on the basis of the $C(p)$ value from the $\mathrm{DCH}$. Such tracks are rejected if: 


$$
\begin{aligned}
& C(\pi) \leq \quad 3.5-2.9(p / 0.4) \quad(p \leq 0.4 \mathrm{GeV} / c) \\
& C(\pi) \leq \quad 0.6 \quad(0.4 \leq p \leq 0.6 \mathrm{GeV} / c) \\
& C(\pi) \leq 0.6-0.8([p-0.6] / 0.4) \quad(0.6 \leq p \leq 1.0 \mathrm{GeV} / c)
\end{aligned}
$$

Since most of the protons in the selected sample have momentum less than $1 \mathrm{GeV} / c$, it was decided not to use DIRC information in the somewhat unreliable threshold region above $1 \mathrm{GeV} / c$ in an attempt to recover class 2 and class 3 tracks which were really protons but which had been rejected because of their $C(\pi)$ value (criterion (b) above).

\subsubsection{Pion Identification}

The positively- and negatively-charged tracks forming the $K_{S}^{0}$ candidates are divided into three classes just as for the proton candidates. The $d E / d x$ versus lab. momentum scatterplots for these tracks are shown in fig. 4.14(a) for tracks with SVT information, and in fig. 4.14(b) for those with DCH information, where we plot the charge-signed lab. momentum on the $x$-axis. The band structure observed for the positive tracks is very similar to that seen in fig. 4.10 , but for the negative tracks the $\bar{p}$ band is much weaker than that for the proton, and there is no evidence of $\bar{d}$ and $\bar{t}$ bands.

The pion bands are concentrated mainly at low momentum, and so again only $d E / d x$ information is used for identification. The pion consistency, $C\left(\pi^{ \pm}\right)$, is required be within the specified tolerance and any surviving track which satisfies baryon or anti-baryon consistency requirements is rejected. This simple procedure greatly improves $K_{S}^{0}$ signal-tobackground without causing much loss of signal.
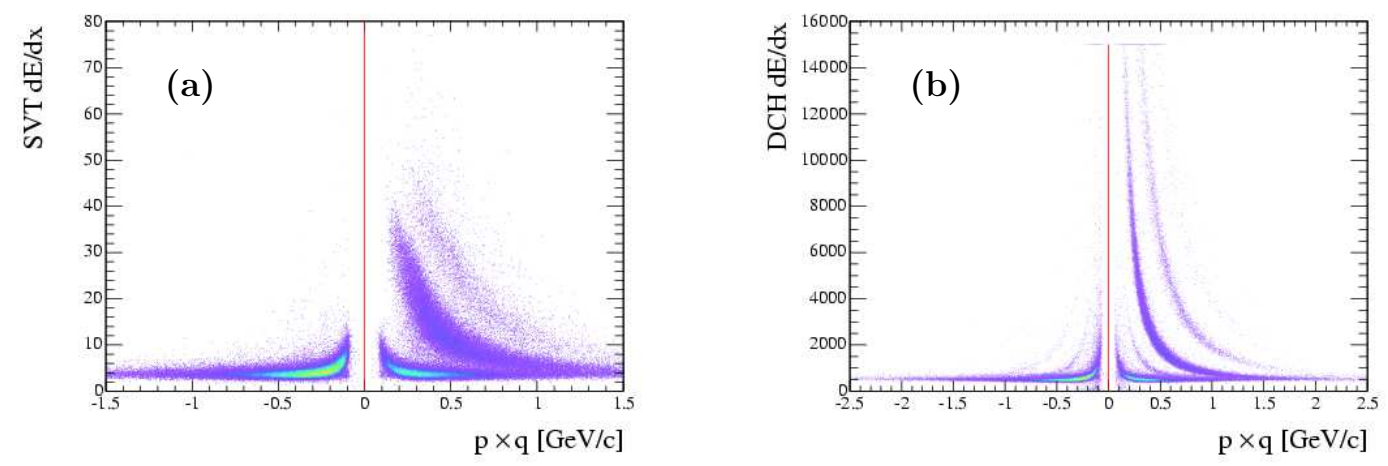

Figure 4.14: (a) The distribution of $d E / d x$ measured in the SVT versus lab. momentum for the tracks forming a candidate $K_{S}^{0}$; the lab. momentum has been given the charge sign of the track; $\pi^{+}, \pi^{-}, p$, and $d$ bands are clear, and there is some faint evidence for $t, K^{+}$, $K^{-}$and $\bar{p}$; (b) the corresponding plot for $d E / d x$ measured in the $\mathrm{DCH}$; as a result of the better resolution, all of the particle bands are observed clearly. 

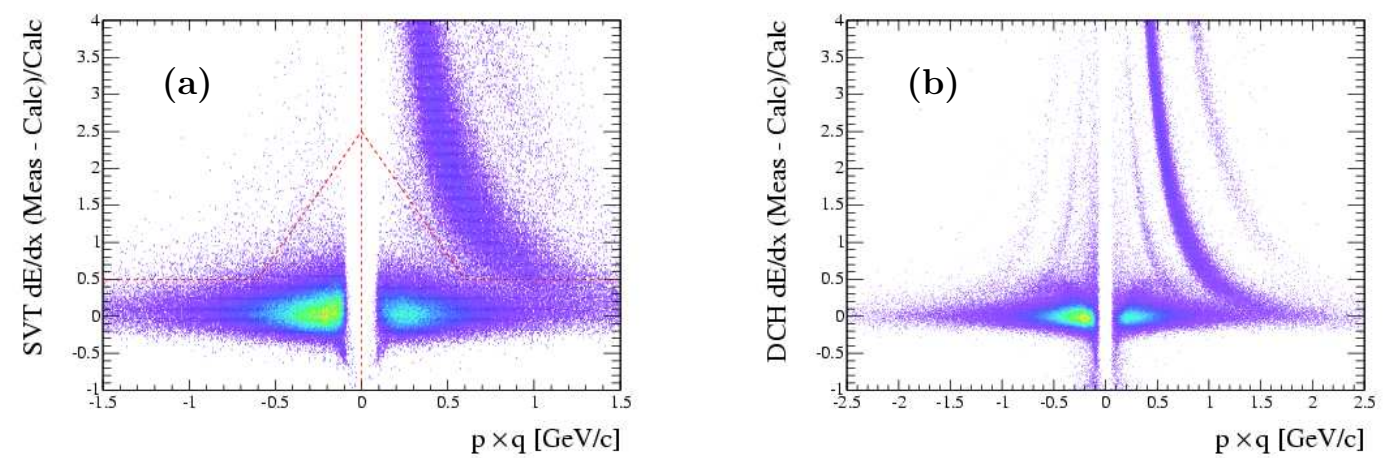

Figure 4.15: (a) The distribution of $C(\pi)$ versus charge-signed momentum for $K_{S}^{0}$ candidate daughter tracks with SVT $d E / d x$ information; (b) the corresponding distribution for tracks with DCH $d E / d x$ information. Class 1 tracks above the dashed red lines in (a), and Class 2 and 3 tracks above the dashed red lines in (b) are rejected as pion candidates.

The distributions of pion consistency, $C(\pi)$, versus lab. momentum which correspond to fig 4.14 are shown in fig 4.15. Class 1 tracks above the red dashed boundary lines in fig 4.15(a), and class 2 and 3 tracks above the dashed lines in fig 4.15(b) are rejected as pion candidates. Pion candidates are retained if they satisfy the following criteria:

(a) For tracks with only SVT coords (i.e. class 1):

$$
\begin{array}{ccc}
C(\pi) \leq & 2.5-2.0(p / 0.6) & (p \leq 0.6 \mathrm{GeV} / c) \\
C(\pi) \leq & 0.5 & (p \geq 0.6 \mathrm{GeV} / c) .
\end{array}
$$

(b) For tracks with DCH coords (i.e. class 2 and 3):

$$
\begin{array}{lcc}
C(\pi) \leq & 2.5-2.2(p / 0.4) & (p \leq 0.4 \mathrm{GeV} / c) \\
C(\pi) \leq & 0.3 & (p \geq 0.4 \mathrm{GeV} / c) .
\end{array}
$$

The selected $\pi^{+}$candidate sample contains a residual proton contamination. For these tracks, the proton consistency, $\mathrm{C}(\mathrm{p})$, is shown as a function of lab. momentum in fig. 4.16(a) for tracks with SVT $d E / d x$ information and in fig. 4.16(b) for those with DCH information. In fig. 4.16(a) there appears to be proton contamination at low momentum. Candidate $\pi^{+}$tracks are removed if:

$$
C(p) \geq-0.85+1.85(p / 1.4) \quad \text { (for all momentum values.) }
$$



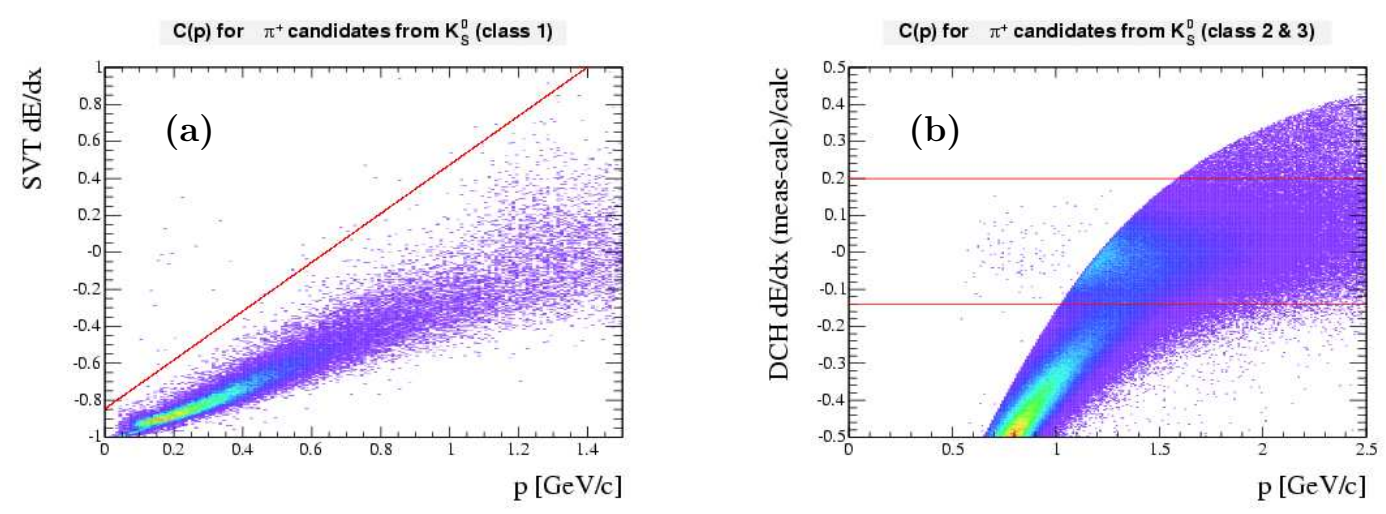

Figure 4.16: (a) The distribution of $C(p)$ versus momentum for the $K_{S}^{0}$ candidate daughter $\pi^{+}$tracks with SVT $d E / d x$ information; (b) the corresponding plot for the $\pi^{+}$tracks with DCH information. Class 1 tracks falling above the line in (a) are rejected; class 2 and 3 tracks falling in the proton band near zero in (b) are rejected.

In fig. 4.16(b), there is an enhancement in the proton band for momenta just above 1 $\mathrm{GeV} / c$. We therefore reject these $\pi^{+}$candidates of class 2 and 3 for which

$$
-0.14 \leq C(p) \leq 0.20
$$

Since most $\pi^{+}$tracks are localized at low momentum, no attempt is made to use the DIRC to recover any real $\pi^{+}$tracks removed by this requirement.

\subsubsection{Tightening the $\left(K_{S}^{0}, p\right)$ Candidate Selection Criteria}

After rejecting each track for which the PID information is inconsistent with the mass assignment made during the initial selection of $\left(K_{S}^{0}, p\right)$ candidates, the initial loose geometrical criteria were revisited, and systematically modified as follows:

- For the $K_{S}^{0}$ candidate, the $\operatorname{DOCA}\left(\pi^{+}, \pi^{-}\right)$value was required to be less than 0.3 $\mathrm{cm}$, and in addition the chi-squared probability, $\mathrm{P}\left(\chi^{2}\right)$, of the vertex fit had to satisfy $\mathrm{P}\left(\chi^{2}\right)>0.001$; the mass distribution of fig $4.17(\mathrm{a})$ is obtained after requiring DOCA $<0.3 \mathrm{~cm}$, and the DOCA distribution for this region is shown in $0.01 \mathrm{~cm}$ bins in fig 4.17(b); the lower distribution in each of the plots shows the distribution of the $K_{S}^{0}$ candidates for which $\mathrm{P}\left(\chi^{2}\right)<0.001$; approximately $1 \%$ of the signal was lost as a result of these tighter criteria.

- The DOCA between the proton candidate and the $K_{S}^{0}$ flight path was required to be less than $0.3 \mathrm{~cm}$;

- The $K_{S}^{0} \mathrm{SFL}$ was required to be greater than $0.2 \mathrm{~cm}$.

The net effect on the distribution of $K_{S}^{0}$ candidate helicity cosine is shown in fig. 4.18(a). The peak due to Lambda reflection and the strong slope toward $\cos \Theta_{H}=1$ observed in 

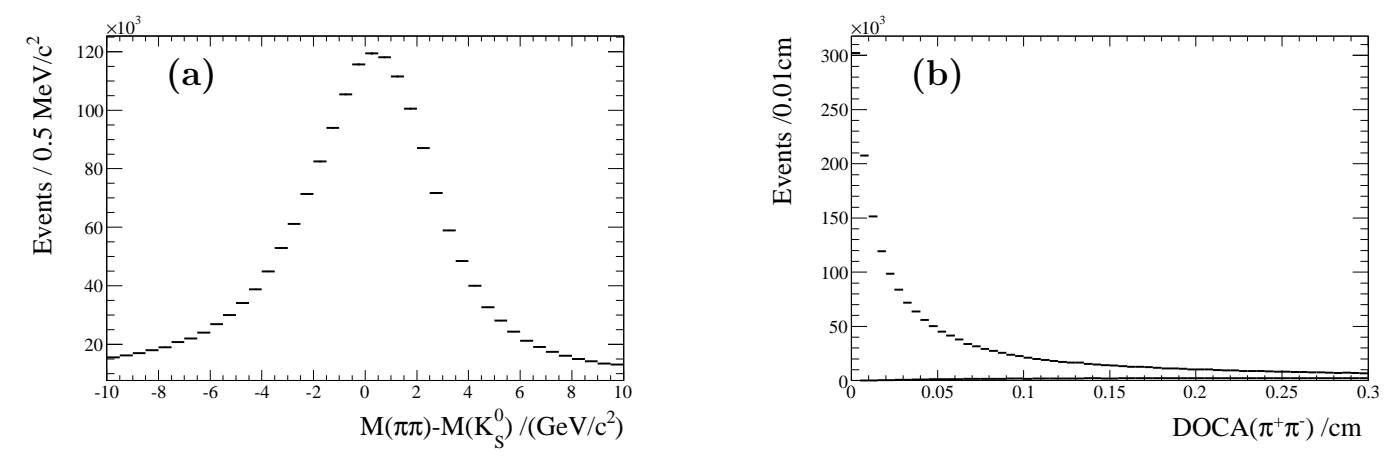

Figure 4.17: (a) The $\pi^{+} \pi^{-}$mass distribution relative to the PDG $K_{S}^{0}$ mass after requiring $\operatorname{DOCA}\left(\pi^{+}, \pi^{-}\right)<0.3 \mathrm{~cm}$; (b) the DOCA distribution for the events in (a); in each plot the lower distribution corresponds $\mathrm{P}\left(\chi^{2}\right)<0.001$.

fig. 4.12(a) are no longer present, and the forward and backward spikes due to electron pairs can now be seen quite clearly. The latter are removed by requiring $\left|\cos \Theta_{H}\right|<0.95$. The dips observed in the forward and backward regions are due to the loss of soft and low-transverse-momentum pion tracks, which cannot be reconstructed in the detector.

The final $\pi^{+} \pi^{-}$invariant mass distribution for all $K_{S}^{0}$ candidates is shown in fig. 4.18(b) relative to the PDG $K_{S}^{0}$ mass value [9]. The peak position is consistent with the expected mass, and the half-width-at-half-maximum (HWHM) is $\sim 3 \mathrm{MeV} / c^{2}$. The signal region is defined by

$$
\left|m\left(\pi^{+} \pi^{-}\right)-m\left(K_{S}^{0}\right)\right|<10 \mathrm{MeV} / c^{2}
$$

and the sideband regions are defined by

$$
35<\left|m\left(\pi^{+} \pi^{-}\right)-m\left(K_{S}^{0}\right)\right|<45 \mathrm{MeV} / c^{2} .
$$

The background distribution is linear to a good approximation, and so the events from the combined sideband regions are considered to represent reliably the background contribution in the signal region. In all subsequent plots described as "sideband-subtracted" (SBS) the events from these sidebands have been used to correct for background under the $K_{S}^{0}$ signal by simple subtraction of the corresponding distributions.

The ratio of the number of signal events in the signal region $(\mathrm{S})$ to the number of background events in the signal region (B) is then obtained as

$$
\frac{S}{B}=\frac{\# \text { events in signal region }}{\# \text { events in sidebands }}-1
$$

For fig. 4.18(b), the overall value of $\mathrm{S} / \mathrm{B}$ is $\sim 4.4: 1$, and the variation with $K_{S}^{0}$ SFL is as shown in fig. 4.19. At $0.2 \mathrm{~cm}$, the ratio is $\sim 0.23: 1$, but increases rapidly to $\sim 10: 1$ by $2.0 \mathrm{~cm}$ and seems to level off at a limiting value of $\sim 12: 1$. Although the value at small 

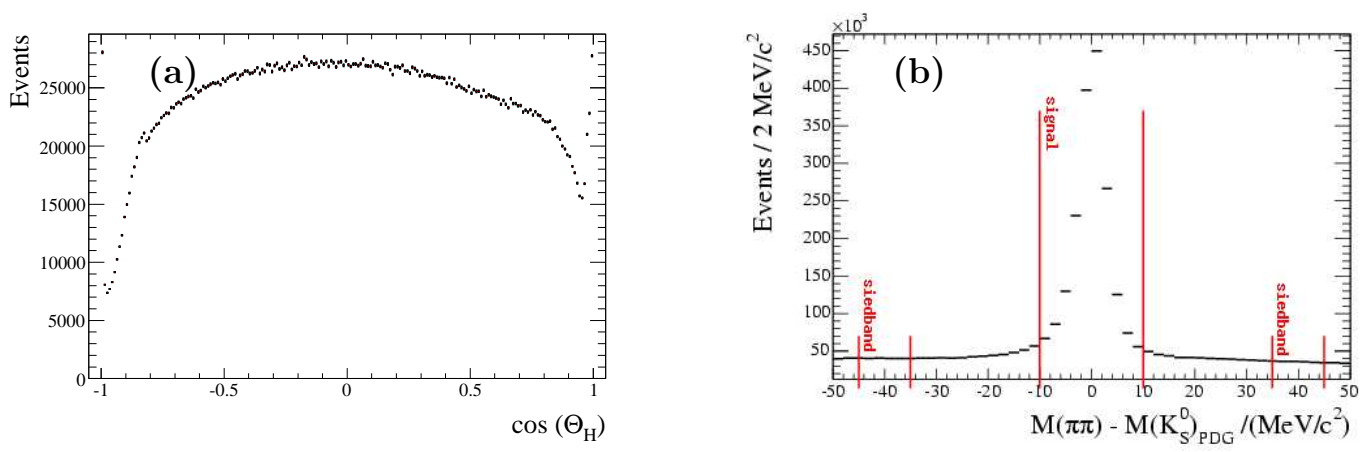

Figure 4.18: (a) The distribution in $\cos \Theta_{H}$ for all $K_{S}^{0}$ candidates after the PID and geometrical selection criteria described in the text, and (b) the $K_{S}^{0}$ candidate mass distribution relative to the PDG value for the sample in (a) after requiring $\left|\cos \Theta_{H}\right|<0.95$ to remove electron pairs; the $K_{S}^{0}$ signal and sideband regions are indicated.
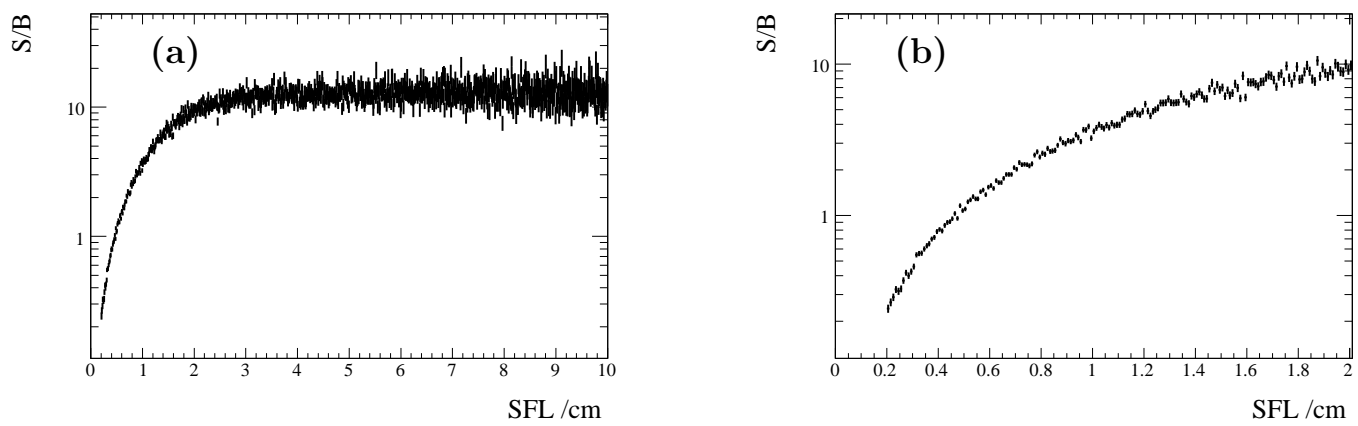

Figure 4.19: (a) The dependence of S/B as defined in eq.(4) on $K_{S}^{0}$ SFL; (b) the distribution of (a) for SFL $<2 \mathrm{~cm}$.

SFL values is not large, this region contains a large number of real $K_{S}^{0}$ mesons, and so we retain candidates in this region and rely on SBS to remove the background contributions. 


\subsection{Detector Tomography Using $K_{S}^{0} p$ Vertices}

The extent to which the production vertices of the final sample of $\left(K_{S}^{0}, p\right)$ candidates reproduce the structure of the $B A B A R$ detector is now investigated. It is necessary to take account of the fact that two coordinate frames are involved. The standard BABAR coordinate system is defined w.r.t. the DCH. However the Support Tube and contents constitute a separate detector system which is subject to mechanical, thermal and magnetic stresses which cause its position and orientation relative to the $\mathrm{DCH}$ to vary slightly with time. In order to relate the SVT and DCH frames a set of so-called Global Alignment (GA) parameters is obtained for each run during data-taking by matching tracks reconstructed in each system separately at the Support Tube wall. This defines the parameters (6 in all) of a rigid body translation and rotation which relate the two frames. In this way local SVT measurements are converted to the DCH frame for use by the general track reconstruction software. For investigation of the $\mathrm{DCH}$ inner cylinder it is appropriate to be in the DCH frame, but for the geometry of the Support Tube and contents a significant improvement in precision is obtained by inverting the GA transformation in order to perform the investigation in the local SVT coordinate system. A further complication results from the fact that the Support Tube was removed from the detector, dismantled, and the SVT dismounted in summer, 2002 between Run Periods 2 and 3. This was done in order to introduce additional cooling in the forward and backward beampipe regions. As a consequence of this, it is necessary to study the structure of the Support Tube and contents separately for Run Periods $1+2$ and $3+4$.

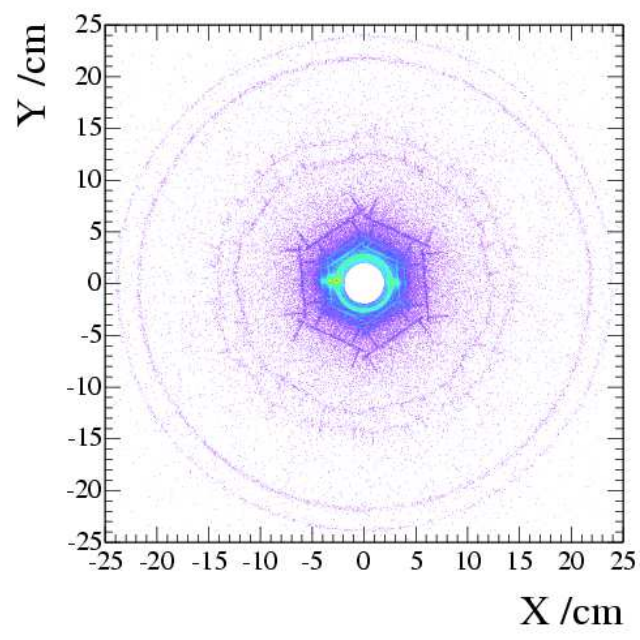

Figure 4.20: The $x-y$ scatter plot for $\left(K_{S}^{0}, p\right)$ vertices from Run Periods 1 and 2 with $z$ coord. satisfying $-7.5 \leq \mathrm{z} \leq 9.5 \mathrm{~cm}$; this restricts the vertices to the $z$ range between the Ta foils of fig. 4.4, and also excludes the lampshade regions of SVT layers 4 and 5 (see fig.4.6).

In order to investigate the tomography of the detector the $K_{S}^{0}$ signal region is selected, 
but SBS is not performed on the resulting scatterplots.

In fig. 4.20 the $x-y$ scatterplot (SVT coords.) is shown for $\left(K_{S}^{0}, p\right)$ vertices from Run Periods $1+2$ for which $-7.5<z<9.5 \mathrm{~cm}$; this corresponds to the $z$ range between the Ta foils visible in fig. 4.4, and so excludes the lampshade regions of SVT layers 4 and 5 (cf. fig. 4.6). The outer circle in the plot corresponds to the Be inner cylinder of the DCH (cf. fig. 4.8; nominal mid-volume radius $23.85 \mathrm{~cm}$.), and the inner circle to the $\mathrm{C}$ fiber section of the Support Tube (cf. fig. 4.7(c); nominal mid-volume radius 21.8 $\mathrm{cm})$. The polygonal structure and support ribs of SVT layers 4 and 5 can be seen, as can the hexagonal structure and ribs of layer 3 . The point density obscures the innermost structure, although a negative- $x$ hot spot corresponding to that in fig. 4.1 is visible. The central hole results from the $2 \mathrm{~cm}$ minimum radius requirement imposed on all candidate vertices.

The inner region of fig. 4.20 is shown in fig. 4.21(a). The overlapping hexagonal structure and radial support ribs of SVT layers 1-3 are now quite clear, and the entire reconstructed geometry of layers 1-5 is in excellent quantitative agreement with the $x-y$ projection of fig. 4.6. Close inspection also reveals the inner SVT r.f. shield at the expected radius of $3.2 \mathrm{~cm}$. The beampipe is the annular region ranging in radius from $\sim 2.5$ to 2.8 cm as expected (c.f. fig. 4.5), and the hot spots of fig. 4.1 are now clear in fig. 4.21(a) also. Hot spots of lower intensity can be seen also in SVT layers 1 and 2; the "trajectories" formed by connecting the negative- and positive- $x$ hot spots are quite consistent with the interpretation in terms of the bending of off-beam $e^{-}$and $e^{+}$illustrated in fig. 4.2.

The $r-z$ scatterplot of $\left(K_{S}^{0}, p\right)$ vertices is shown in fig. 4.21(b) for radii less than 5.5 $\mathrm{cm}$ and $|z|<25 \mathrm{~cm}$. In the central region of $z$, corresponding to fig. 4.21(a), horizontal bands due to the beampipe, SVT r.f. shield, and the hot spots in layers 1 and 2 can be seen. Near $z \sim-8 \mathrm{~cm}$ and $+10 \mathrm{~cm}$ at $r \sim 2.8 \mathrm{~cm}$, there are sharply-defined, high-intensity regions generated by the Ta foils of fig. 4.4. The concentration at $z \sim-15 \mathrm{~cm}$ and $\mathrm{r} \sim$ $2.9 \mathrm{~cm}$ results from the stainless steel flange to which the beampipe cooling-water lines attach, and that at $z \sim-18 \mathrm{~cm}$ and $r \sim 2.6 \mathrm{~cm}$ is due to the stainless steel bellows at which the beampipe is welded to the vacuum chamber. These structures can be seen in fig. 4.4 just beyond the backward Ta foil.

In figs. 4.21(c) and (d) we show the $x-y$ scatterplots for the Ta foil regions, -10.0 $<z<-7.5 \mathrm{~cm}$ and $9.5<z<11.0 \mathrm{~cm}$ resp. For both regions the $\left(K_{S}^{0}, p\right)$ vertices are concentrated in the region of the negative- $x$ hot spot, with the intensity at negative $z$ being much higher.

For Run Periods 3+4, the distributions corresponding to figs. 4.20 and 4.21 are indistinguishable from those for Run Periods $1+2$ with the exception of fig. 4.21(a). Figure 4.21(a) is reproduced in fig. 4.22(a) and compared with the corresponding distribution for run periods $3+4$ in fig. $4.22(\mathrm{~b})$. The SVT layer structure is virtually identical at this level of precision in both plots, but the hole due to the $2 \mathrm{~cm}$ minimum radius requirement is not the same. In fact the hole appears to be shifted horizontally by $1-2 \mathrm{~mm}$ towards positive $x$ 

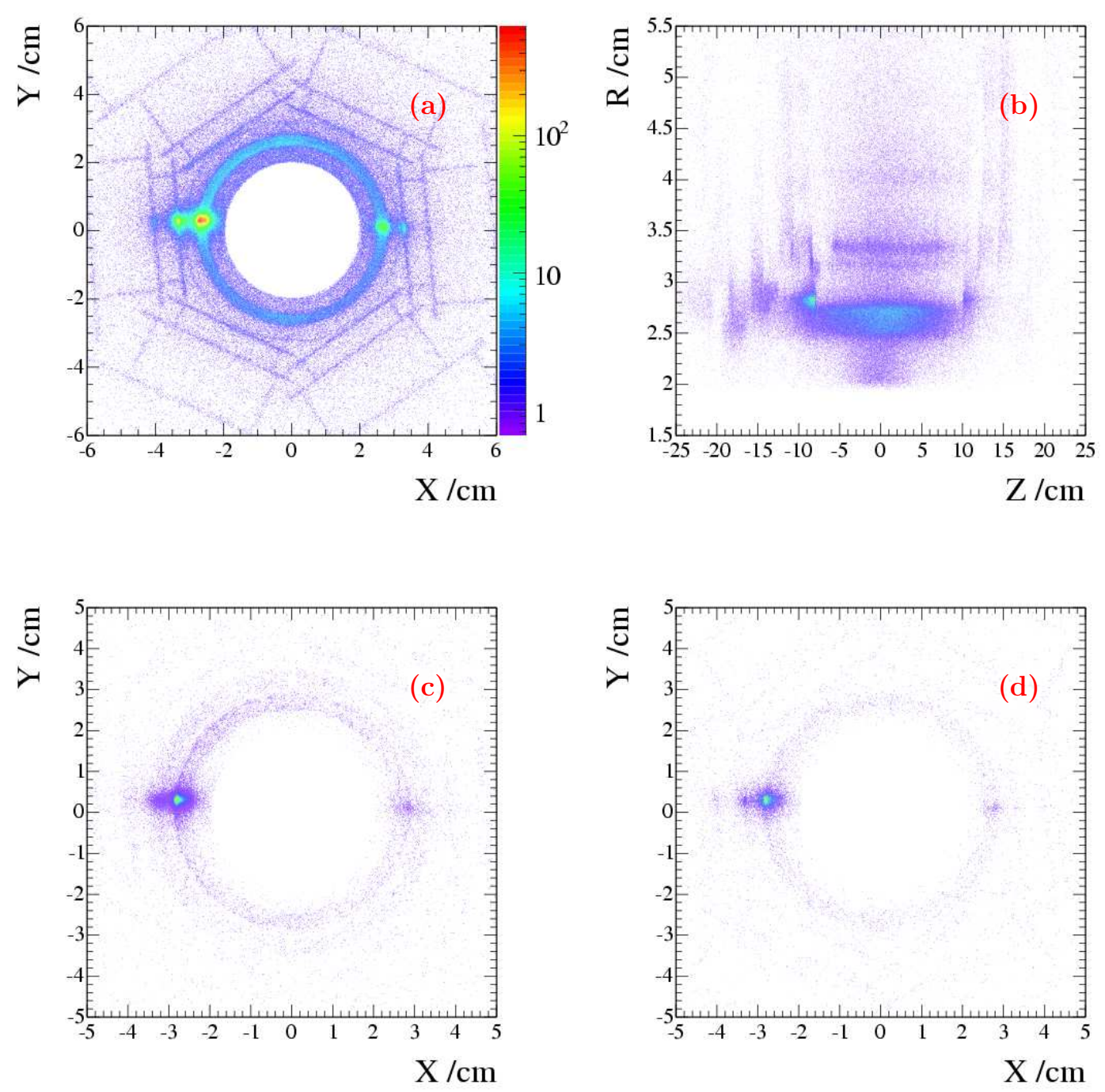

Figure 4.21: (a) The $x-y$ scatterplot for the inner detector region of fig. 4.20 (Run Period $1+2$, SVT coords.); (b) the $r-z$ scatterplot from the same sample of $\left(K_{S}^{0}, p\right)$ vertices for $r<5.5 \mathrm{~cm}$ and $|z|<25 \mathrm{~cm}$; (c) the $x-y$ scatterplot for the backward Ta foil region $(-10.0$ $<z<-7.5 \mathrm{~cm}$ ) of fig. 4.21(b); (d) the $x-y$ scatterplot for the forward Ta foil region $(9.5$ $<z<11.0 \mathrm{~cm}$ ) of fig. 4.21(b); the structures observed in (a)-(d) are discussed in the text.

for Run Periods $3+4$. This means that the SVT itself is shifted toward negative $x$ relative to the DCH by $1-2 \mathrm{~mm}$ for Run Periods $3+4$ compared to its location during Run Periods $1+2$. Clearly, if the data from Run Periods 1-4 were combined in the DCH frame of reference the SVT structure would be made less clearly defined than it is after inverting the GA and examining it in the local SVT coordinate system.

The scale of the following plots examining other aspects of the detector geometry is such that the Run Period differences are not apparent. Consequently the $\left(K_{S}^{0}, p\right)$ data samples for Run Periods 1-4 are combined.

In fig. 4.23 vertex $r$ versus $z$ is plotted for $15 \leq \mathrm{r} \leq 30 \mathrm{~cm}$ and $-110 \leq \mathrm{z} \leq 130 \mathrm{~cm}$ in the DCH frame. The horizontal band at $\mathrm{r} \sim 24 \mathrm{~cm}$ is the inner cylinder of the DCH. 

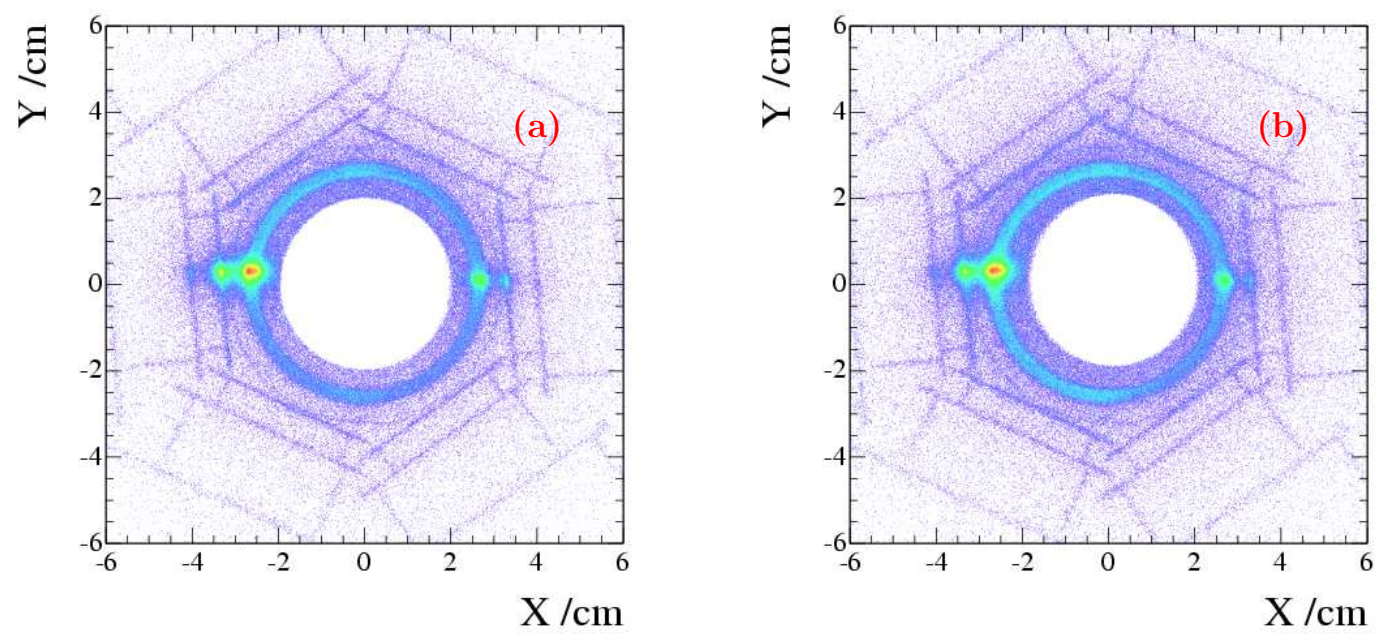

Figure 4.22: (a) The $x-y$ scatterplot of fig. 4.21(a); (b) the same plot for Run Periods $3+4$. For the latter, the circle in the center is shifted toward positive $x$ and distorted.

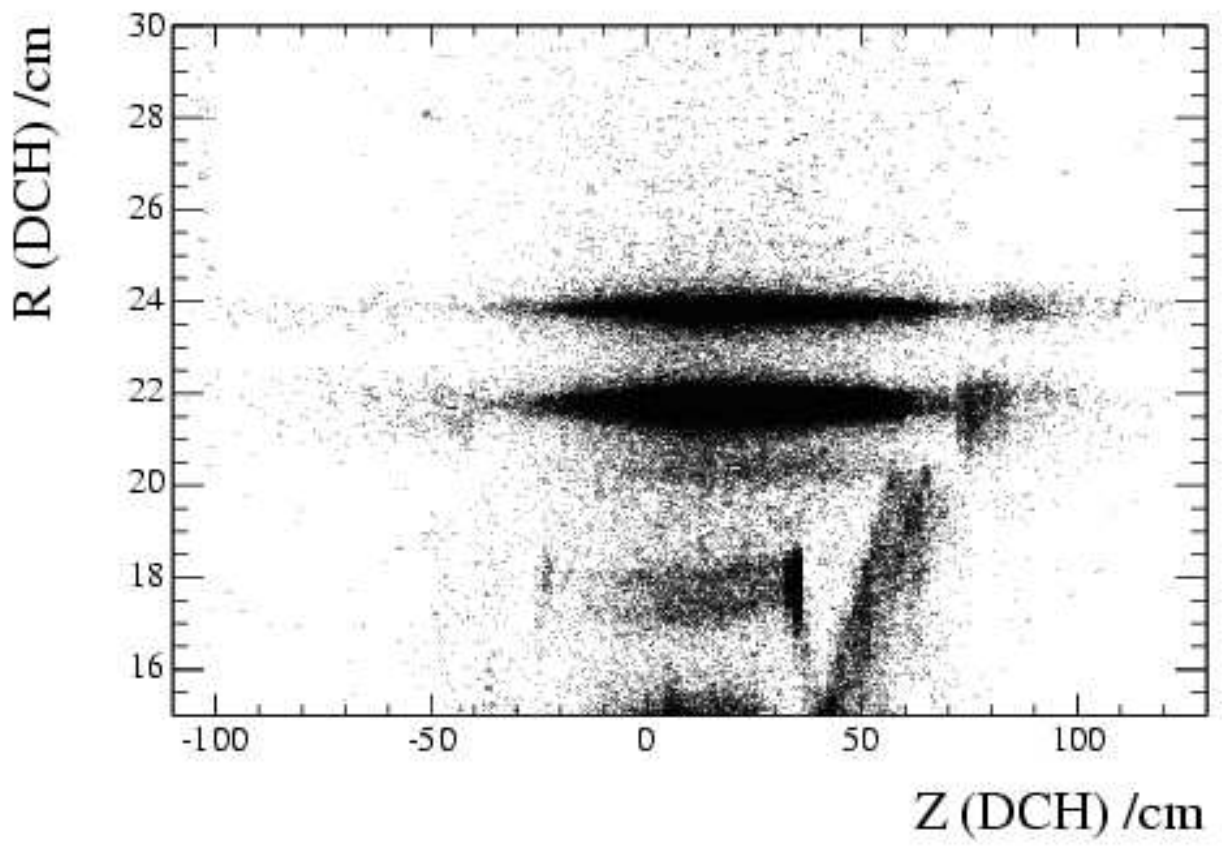

Figure 4.23: The $\left(K_{S}^{0}, p\right)$ candidate vertex $r$ versus $z$ distribution for the combined data from Run Periods 1-4. The structures apparent in the plot are discussed in the text.

The central Be section ( $1 \mathrm{~mm}$ thick) extends from $z=-54.6 \mathrm{~cm}$ to $z=+80.3 \mathrm{~cm}$; the intensity increase at $z \sim+80 \mathrm{~cm}$ indicates the beginning of the forward $\mathrm{Al}$ cylinder $(5 \mathrm{~mm}$ thick), and there is a corresponding faint increase at $\sim-55 \mathrm{~cm}$ due to the junction with the backward $\mathrm{Al}$ cylinder. Continuing to negative $z$, there is a faint radial distribution of vertices at $z \sim-100 \mathrm{~cm}$; this is interpreted as being due to the upstream DCH endplate. 

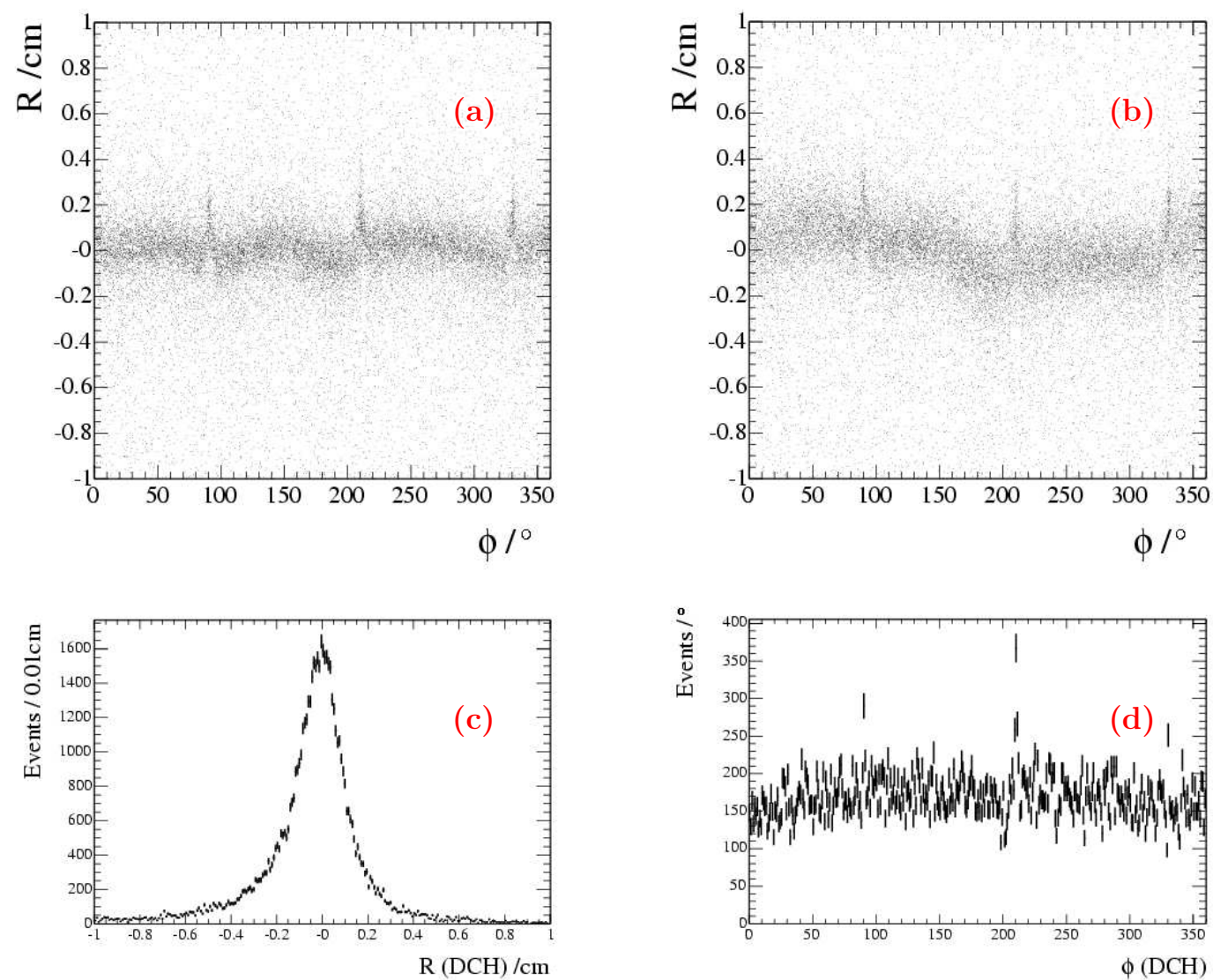

Figure 4.24: The scatter plot of $(\mathrm{R}-23.85) \mathrm{cm}$ versus phi for $\left(K_{S}^{0}, p\right)$ vertices with $(-54.6 \leq \mathrm{z} \leq 80.3 \mathrm{~cm})$ and $\mathrm{R}$ within $1 \mathrm{~cm}$ of the nominal mean radius of the DCH inner Be wall; the plot is in the DCH coordinate frame; (b) the same as (a) but in the SVT frame; (c) the projection of (a) onto $(\mathrm{R}-23.85) \mathrm{cm}$, and (d) the projection of (a) onto $\phi$. The structures are discussed in the text.

There are no vertices forward of $z=130 \mathrm{~cm}$ (the forward endplate is at $z=176 \mathrm{~cm}$ ).

The horizontal band at $r \sim 22 \mathrm{~cm}$ is due to the Support Tube. The central carbon fibre section $(2 \mathrm{~mm}$ thick) extends from $z=-40 \mathrm{~cm}$ to $z=+73 \mathrm{~cm}$. The intensity increase at $z \sim 73 \mathrm{~cm}$ marks the beginning of the forward stainless steel cylinder ( $\sim$ half-inch thick), and there is a faint intensity increase at $z \sim-40 \mathrm{~cm}$ due to the similar transition to the backward stainless steel cylinder.

The faint horizontal band near $r \sim 20 \mathrm{~cm}$ is due to the outer SVT r.f. shield (see fig. 4.7(b)); the region of increased intensity near $r \sim 18 \mathrm{~cm}$ extending to $z \sim+30 \mathrm{~cm}$ is due to the carbon fibre tubes of the SVT support structure, and the dark blob at $z \sim 30$ $\mathrm{cm}$ results from a brass support ring. The diagonal region from $r \sim 15 \mathrm{~cm}, z \sim 40 \mathrm{~cm}$ to $r \sim 20 \mathrm{~cm}$ and $z \sim 55 \mathrm{~cm}$ is due to SVT services, cabling and readout cards.

In fig. 4.24 the DCH Be inner wall $(-54.6 \leq z \leq 80.3 \mathrm{~cm})$ is examined in some detail. The $\mathrm{R}$ versus $\phi$ scatter plot for $\left(K_{S}^{0}, p\right)$ vertices within $\pm 1 \mathrm{~cm}$ of the nominal radius is shown in fig. 4.24(a) in the $\mathrm{DCH}$ coordinate system, and in fig. 4.24(b) in the SVT coordinate frame; fig. 4.24(c) is the projection of fig. $4.24(\mathrm{a})$ onto (R-23.85) $\mathrm{cm}$ and 
fig. 4.24(d) is that of fig. 4.24(a) onto phi. Comparing fig. 4.24(a) and fig. 4.24(b), the effect of the offset and time dependence of the SVT coordinate system can be seen quite clearly; the azimuthal dependence of the wall structure is more pronounced and less clear in fig. 4.24(b) than in fig. 4.24(a). The cylinder was made of three Be sheets, shaped and brazed together by means of Be ribs at $90^{\circ}, 210^{\circ}$ and $330^{\circ}$ in azimuth. In fig. 4.24(a) the rib locations are quite clear, and the structure of each of the three sheets seems to show the same $\sim 1 \mathrm{~mm}$ peak-to-trough undulation. The rib structure is shown in the drawing of fig. 4.25. The ribs extend $1 \mathrm{~mm}$ outward and $1 \mathrm{~mm}$ inward relative to the inner and outer cylinder surfaces. However in fig. 4.24(a), only an outward extension of $\sim 2 \mathrm{~mm}$ can be seen. Inspection of the inner wall of the cylinder shows directly that the ribs do in fact extend inward as designed; the rib structure observed in fig. 4.24(a) is not understood. The radial projection of fig. 4.24 (c) shows that the mean reconstructed radius is in very good agreement with the nominal value, and that the radial vertex resolution $(\sigma)$ is $\sim 1$ $\mathrm{mm}$. The azimuthal distribution of fig. 4.24(d) is rather flat (except for the ribs), and this suggests that the interactions in the Be wall are due to hadrons produced in primary $e^{+} e^{-}$collisions.

The $\mathrm{C}$ fiber section of the Support Tube $(-40 \leq z \leq 73 \mathrm{~cm})$ is investigated in fig. 4.26. The SVT coordinate system is used for fig. 4.26(a) and the DCH frame is used for fig. 4.26(b). The mean radius varies with azimuth in both frames, but the effect is smaller in the SVT frame; this indicates that the Support Tube is off-center in both frames, the magnitude being $\sim 1 \mathrm{~mm}$ in the $x$-direction for the SVT frame. The mean radius is smaller than nominal by $\sim 0.5 \mathrm{~mm}$ (fig. 4.26(c)), and allowing for the thickness of the cylinder (2 $\mathrm{mm})$ and the magnitude of the $\phi$ oscillation, it is again estimated that the radial resolution $(\sigma)$ is $\sim 1 \mathrm{~mm}$. The flatness of the azimuthal distribution (fig. 4.26(d)) again suggests that the interactions are due to hadrons produced in primary $e^{+} e^{-}$collisions.

Finally, the $\mathrm{R}$ versus $\mathrm{Z}$ distribution for $\left(K_{S}^{0}, p\right)$ vertices in the region of SVT layers 4 and $5(9 \leq \mathrm{R} \leq 16 \mathrm{~cm},-25 \leq \mathrm{Z} \leq 35 \mathrm{~cm})$ is examined. The distribution is shown in fig. 4.27(a). The double polygon structure and lampshade regions of layers 4 and 5 can be seen quite clearly, as can the $\mathrm{C}$ fiber endpieces and cooling rings on the surface of the forward B1 magnet. However it was a bit surprising to see regions of increased intensity for both layers for $z$ values $\sim+6 \mathrm{~cm}$; this suggested the presence of extra material, and in fact in fig. 4.27(b) a clip can be seen near the $z$ region in question for each side of the layer 5 polygon. Finally, the engineering drawing of fig. $4.27(\mathrm{c})$ was obtained; this indicated

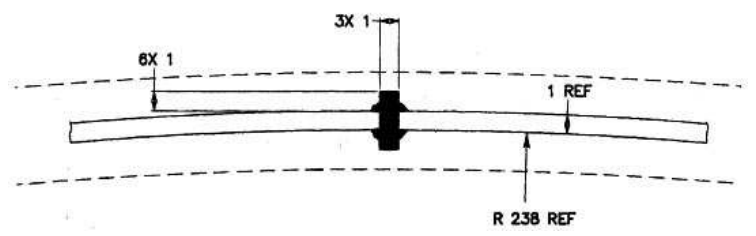

Figure 4.25: Rib structure of the DCH inner wall. 

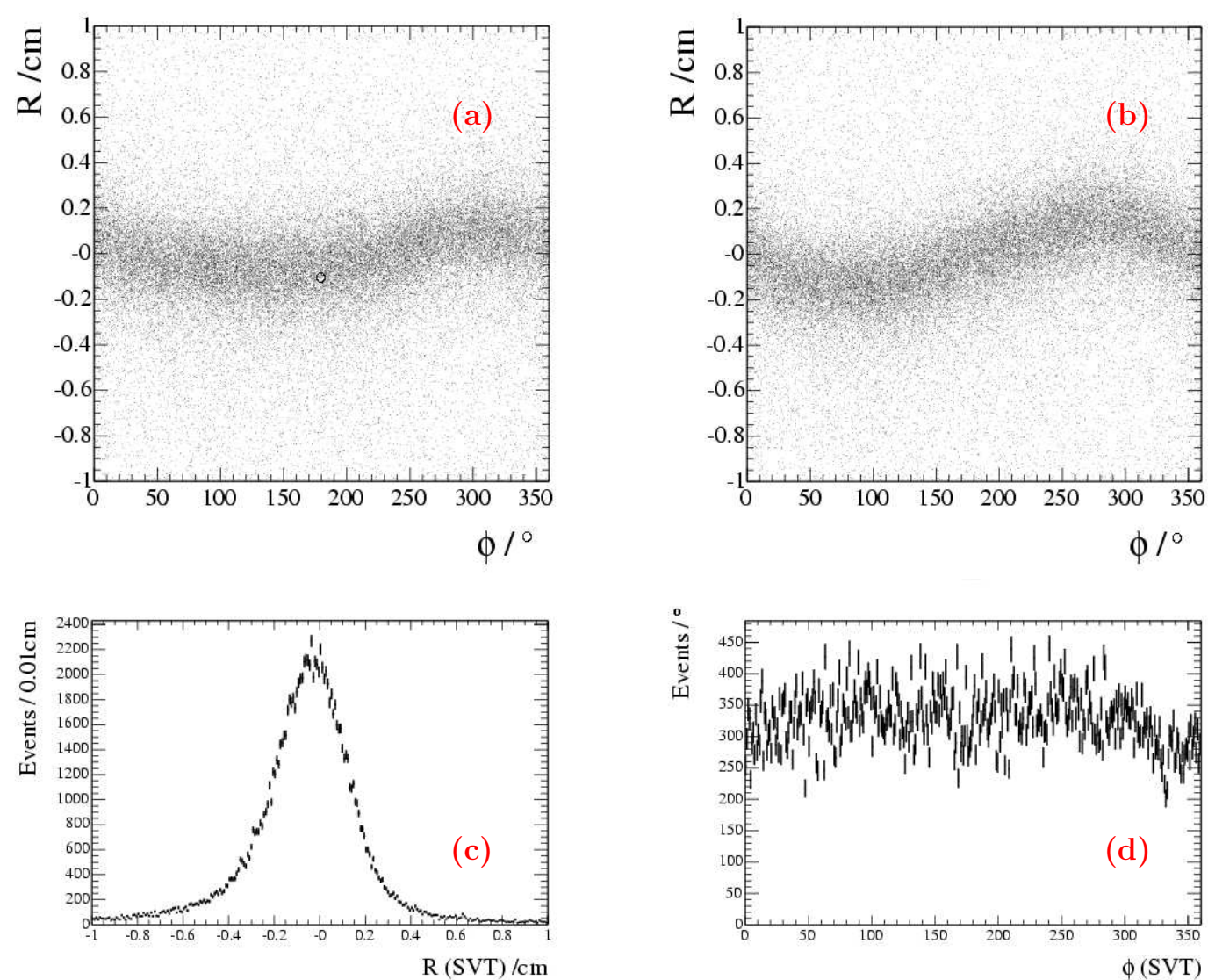

Figure 4.26: The scatter plot of (R-21.8) cm versus phi for $\left(K_{S}^{0}, p\right)$ vertices with $(-40 \leq \mathrm{z} \leq$ $73 \mathrm{~cm}$ ) and $\mathrm{R}$ within $1 \mathrm{~cm}$ of the nominal mean radius of the $\mathrm{C}$ fibre Support Tube; the plot is in the SVT reference frame; (b) the same as (a) but in the DCH frame; (c) the projection of (a) onto $(\mathrm{R}-21.8) \mathrm{cm}$, and $(\mathrm{d})$ the projection of (a) onto $\phi$. The plots are discussed in the text.

that there was a clip made of Kevlar for each side of the layer 4 and 5 polygons, 34 clips in all. These are epoxied to the support ribs for each side to provide increased rigidity near the junction between the wafers being read out at positive $z$ and those being read out at negative $z$. These clips were not included in the material model of the detector but will be added in the near future.

There will be discussion of the material structure observed along the beampipe for ( -25 $\leq z \leq 20 \mathrm{~cm}$ ) in conjunction with the selection of the electroproduction interaction data in Section 4.7 below.

In conclusion, it seems perfectly reasonable to proceed to an examination of the physics of the $\left(K_{S}^{0}, p\right)$ data sample on the basis that, as selected, the candidates result from hadroproduction and electroproduction interactions in the material of the BABAR detector. 


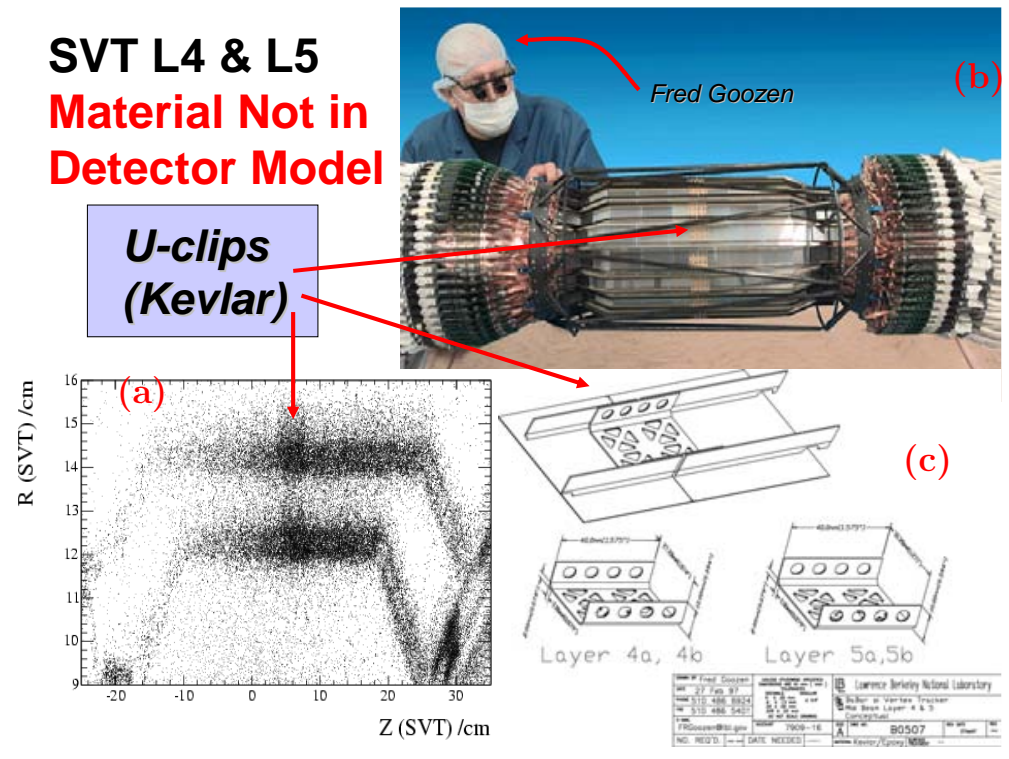

Figure 4.27: (a) The $\mathrm{R}$ vs $\mathrm{Z}$ distribution for $\left(K_{S}^{0}, p\right)$ vertices in the region of SVT Layers 4 and 5; (b) the SVT mounted between the B1 magnets; the Kevlar clips on Layer 5 can be seen quite clearly; (c) an engineering drawing of the Kevlar clips used for SVT layers 4 and 5 . 


\subsection{Search for $\Theta_{5}(1540)^{+} \rightarrow K_{S}^{0} p$ Using all Candidates}

In the search for $\Theta_{5}(1540)^{+}$the combined data sample from Run Periods 1-4 is used; the corresponding $e^{+} e^{-}$integrated luminosity is $\sim 230 \mathrm{fb}^{-1}$. The $K_{S}^{0} p$ invariant mass distribution is restricted to the region from threshold to $1.6 \mathrm{GeV} / c^{2}$. In all mass studies described in this Section and in Section 4.7, the mass region above $1.6 \mathrm{GeV} / c^{2}$ has been examined, but no significant structure of any kind has been found. Consequently, the relevant plots are not shown here; instead they are included in the Appendices.

\subsubsection{The Inclusive $K_{S}^{0} p$ Invariant Mass Distribution}

For a sub-sample of the $\left(K_{S}^{0}, p\right)$ candidates selected as described in Sections 4.4 and 4.5, the SBS $K_{S}^{0}$ and proton lab. momentum distributions are shown in fig. 4.28(a) and fig. 4.28(b) respectively.
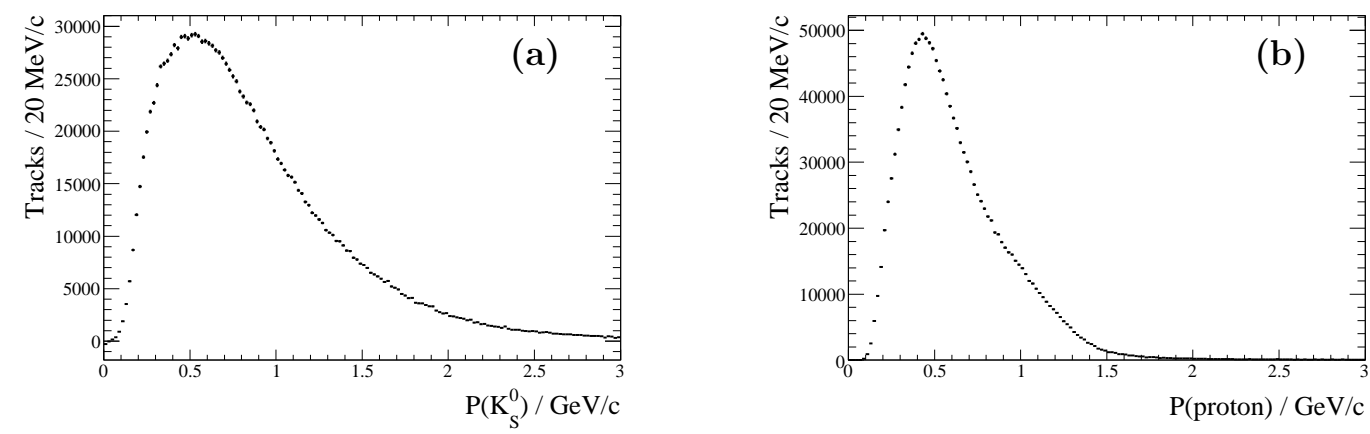

Figure 4.28: (a) The SBS lab. momentum distribution for the $K_{S}^{0}$ from a sub-sample of $\left(K_{S}^{0}, p\right)$ candidates; (b) the corresponding proton lab. momentum distribution.

The $K_{S}^{0}$ distribution peaks at $\sim 0.5 \mathrm{GeV} / c$, extends down to $\sim 0.1 \mathrm{GeV} / c$, falls off rapidly toward high momentum and tails off by $\sim 3 \mathrm{GeV} / c$. The proton distribution is also very soft; it peaks at $\sim 0.4 \mathrm{GeV} / c$, extends down to $\sim 0.1 \mathrm{GeV} / c$, and also decreases rapidly toward high momentum. The region above $\sim 1.2 \mathrm{GeV} / c$ is severely curtailed as a result of the pion/kaon rejection procedure described in Section 4.2 but the distribution is already falling off so rapidly that this should have no significant effect on the analysis of the region of $K_{S}^{0} p$ mass below $1.6 \mathrm{GeV} / c^{2}$.

The $K_{S}^{0} p$ invariant mass distribution near threshold is shown in fig. 4.29; the full mass distribution contains $\sim 1.9$ million candidates. There are $\sim 8500$ candidates $/\left(2 \mathrm{MeV} / \mathrm{c}^{2}\right)$ in the vicinity of the $\Theta_{5}^{+}$mass, where the mass resolution is $\sim 2 \mathrm{MeV} / c^{2}$ [59]. The lower distribution is obtained from the $K_{S}^{0}$ sideband regions. There is no evidence of a $\Theta_{5}^{+}$signal in this data sample, and the sideband contribution is $\sim 20 \%$ of that from the $K_{S}^{0}$ signal region at $\sim 1.54 \mathrm{GeV} / c^{2}$. 


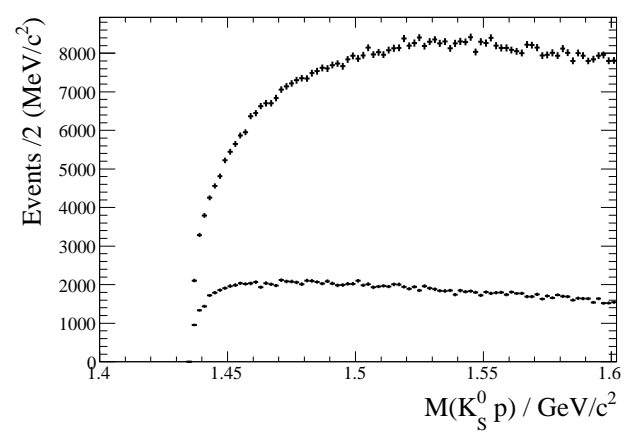

Figure 4.29: The $K_{S}^{0} p$ invariant mass distribution near threshold for all candidate $\left(K_{S}^{0}\right.$, $p$ ) vertices; the upper distribution is for the $K_{S}^{0}$ signal region and the lower is for the $K_{S}^{0}$ sidebands.

\subsubsection{The Inclusion of Additional Charged Tracks}

Recall that the maximum DOCA between a $K_{S}^{0}$ candidate flight path and associated proton is $0.3 \mathrm{~cm}$, and that the mid-point of this DOCA is taken as the approximate position of the production vertex.

In order to increase the reliability of the $\left(K_{S}^{0}, p\right)$ sample, and to investigate other mass combinations involving the candidate $K_{S}^{0}$ and $p$, an attempt is made to associate other charged tracks in an event with each $\left(K_{S}^{0}, p\right)$ candidate vertex in that event; only tracks forming the $\left(K_{S}^{0}, p\right)$ candidate being considered are excluded. The DOCA of each charged track to the $\left(K_{S}^{0}, p\right)$ vertex is found, and the DOCA distribution for values up to $2 \mathrm{~cm}$ is as shown in fig. 4.30(a). Tracks with DOCA $<0.5 \mathrm{~cm}$ are considered to be vertex-associated, and it is required that there be at least one such track for each $\left(K_{S}^{0}, p\right)$ candidate. The data sample is reduced to $\sim 1.2$ million candidates, and the $K_{S}^{0} p$ invariant mass distributions for the $K_{S}^{0}$ signal and sideband regions are as shown in fig. 4.30(b). Again there is no sign of a $\Theta_{5}^{+}$signal, and the $K_{S}^{0}$ sideband contribution is still $\sim 20 \%$ of that from the signal region.

\subsubsection{The Identification of Associated Charged Tracks}

The identities of the charged tracks which have been associated with the $\left(K_{S}^{0}, p\right)$ vertices are now investigated. Class 1 tracks (SVT only) are excluded since their number is small, their $d E / d x$ resolution is at least a factor of two worse than that for DCH tracks, and their momentum resolution is also considerably worse than for DCH tracks. For the remaining class 2 and class 3 tracks, the scatterplot of DCH $d E / d x$ against momentum at DCH entrance is shown in fig. 4.31, where the momentum has the charge-sign of the particle involved; there are $\sim 2.9$ million entries in the plot. Bands associated with $\pi^{ \pm}, K^{ \pm}, p, \bar{p}$, deuteron and triton are quite clear, and even a faint alpha particle band can be seen. The various particle types are selected on the basis of $d E / d x$ consistency as explained below; for pion and kaon selection, additional likelihood-selector information from the DIRC is 

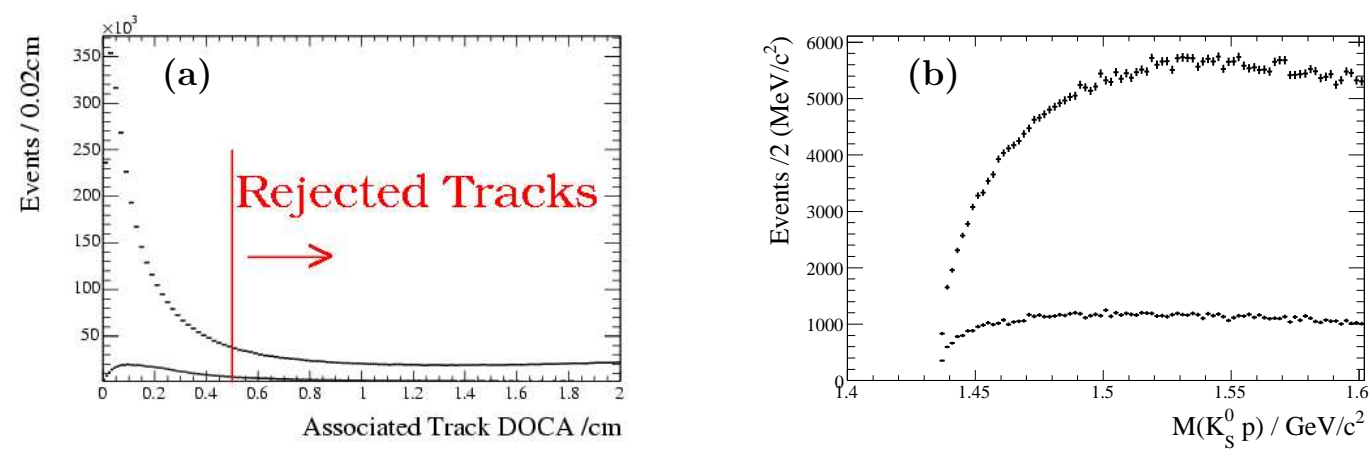

Figure 4.30: (a) The distribution of distance of closest approach (DOCA) to the $p K_{S}^{0}$ vertex; the maximum value for vertex association $(0.5 \mathrm{~cm})$ is indicated. (b) The $K_{S}^{0} p$ invariant mass for all vertices with at least one associated charged track; in both plots the upper distribution is for the $K_{S}^{0}$ signal, the lower for $K_{S}^{0}$ sidebands

incorporated also.

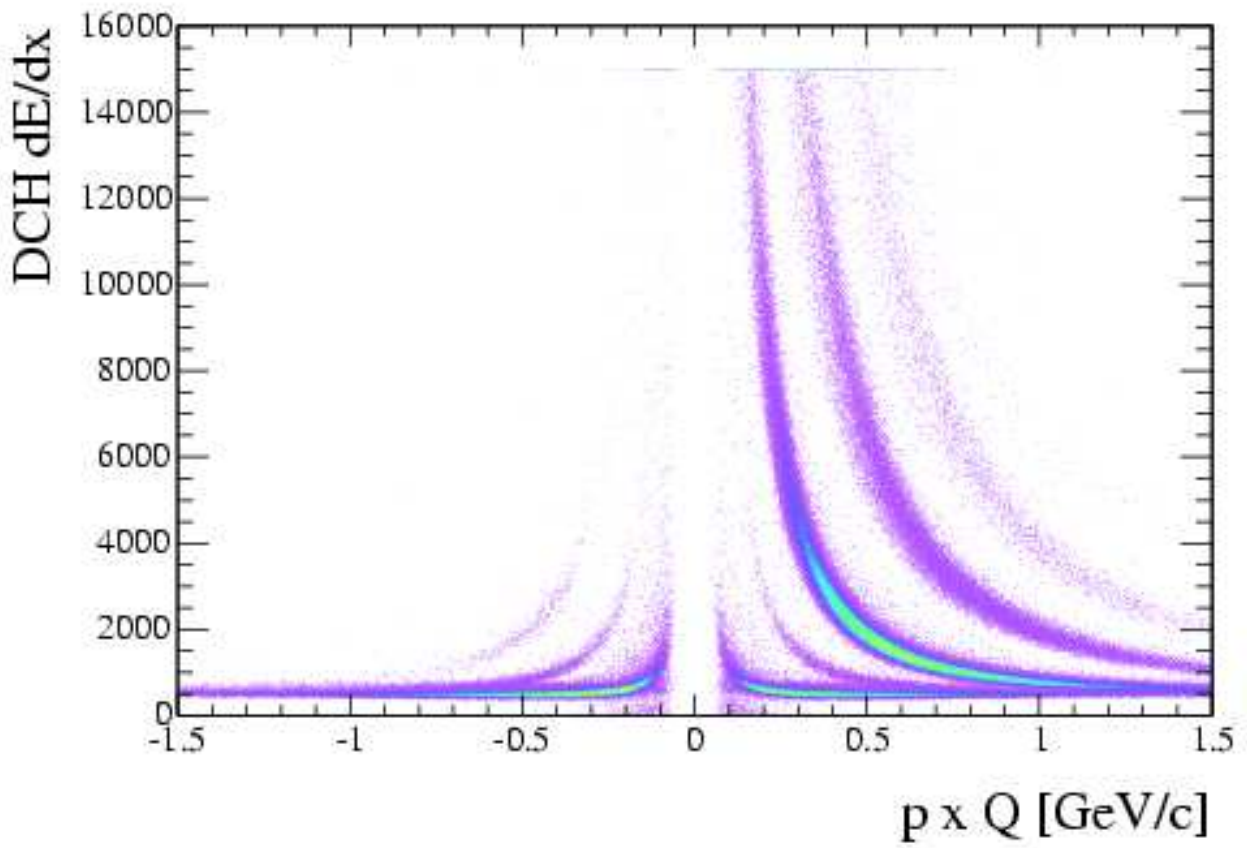

Figure 4.31: The scatterplot of $d E / d x(\mathrm{DCH})$ versus charge-signed momentum for charged tracks associated with a $\left(K_{S}^{0}, p\right)$ vertex and having DOCA $<0.5 \mathrm{~cm}$.

\section{Rejection of $\left(K_{S}^{0}, p\right)$ Candidates with Associated Baryons}

The association of a baryon track with a $\left(K_{S}^{0}, p\right)$ candidate vertex suggests that the production process does not occur on a single virtual nucleon target. The appropriate inference would appear to be that a nuclear-breakup process is involved, or that there 
has been significant final state rescattering. It is reasonable to expect that the $K_{S}^{0} p$ mass distribution might be distorted for such events, and that the probability of observing a $\Theta_{5}^{+}$signal would be enhanced by eliminating them.

For similar reasons the small number of candidates with an associated $\bar{p}$ are removed, since the production mechanism for such events also must involve the production of at least one additional baryon.

For the positively-charged tracks of fig. 4.31 the proton consistency, $C(p)$, and for the negatively-charged tracks that for $\bar{p}, C(\bar{p})$, are plotted against charge-signed momentum in fig. 4.32 .

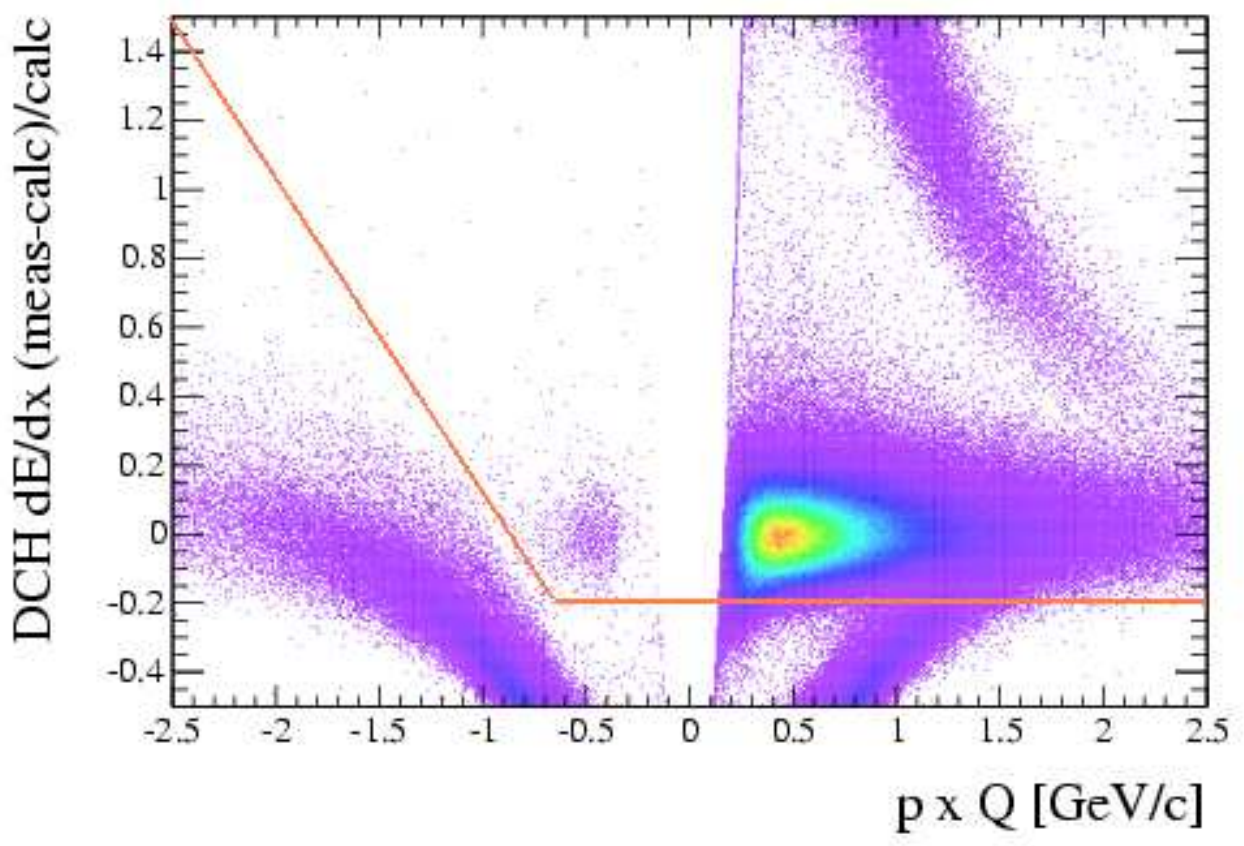

Figure 4.32: The DCH proton or $\bar{p}$ consistency, $C(p$ or $\bar{p})$, versus charge-signed momentum for tracks associated with $\left(K_{S}^{0}, p\right)$ candidate vertices; tracks with $d E / d x$ values above the red lines are considered to be baryons.

The baryon region of fig. 4.32 is defined as follows:

$$
\begin{array}{lcl}
C(p \text { or } \bar{p})> & -0.2 & (p \times Q>-0.65 \mathrm{GeV} / c) \\
C(p \text { or } \bar{p})> & 1.5-\left(\frac{1.7}{1.85}\right)(2.5+p \times Q) & (p \times Q<-0.65 \mathrm{GeV} / c)
\end{array}
$$

and is denoted by the red lines in fig. 4.32; all vertices with at least one associated track satisfying these criteria are rejected.

It should be noted, in particular, that this procedure removes candidate $\left(K_{S}^{0}, p\right)$ vertices 
for which the same $K_{S}^{0}$ candidate is combined with a multi-proton production vertex to yield multiple $\left(K_{S}^{0}, p\right)$ candidates.

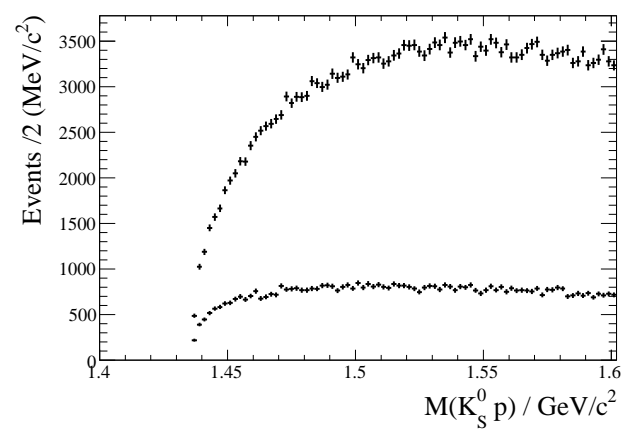

Figure 4.33: The $K_{S}^{0} p$ invariant mass distribution after removing vertices with an associated baryon or $\bar{p}$; the upper distribution is for the $K_{S}^{0}$ signal region, the lower for the $K_{S}^{0}$ sidebands.

The $\left(K_{S}^{0}, p\right)$ sample which survives this baryon removal procedure contains $\sim 720,000$ candidates. For these, the $K_{S}^{0} p$ invariant mass distributions for $K_{S}^{0}$ signal and sideband regions are shown in fig. 4.33. As before, no $\Theta_{5}^{+}$signal is observed, and the sideband contribution is still $\sim 20 \%$ of that from the $K_{S}^{0}$ signal.

\section{$\left(K_{S}^{0}, p\right)$ Candidates with an Associated $\pi^{+}$or $\pi^{-}$}

Candidate vertices with an associated baryon or $\bar{p}$ are first removed. For the selection of $\left(K_{S}^{0}, p\right)$ vertices with an associated $\pi^{+}$or $\pi^{-}$a pion consistency, $C(\pi)$, and a kaon consistency, $C(K)$, are defined using DCH $d E / d x$ information as previously. The corresponding distributions as a function of charge-signed momentum are shown in fig. 4.34(a) and fig. 4.34(b) respectively.
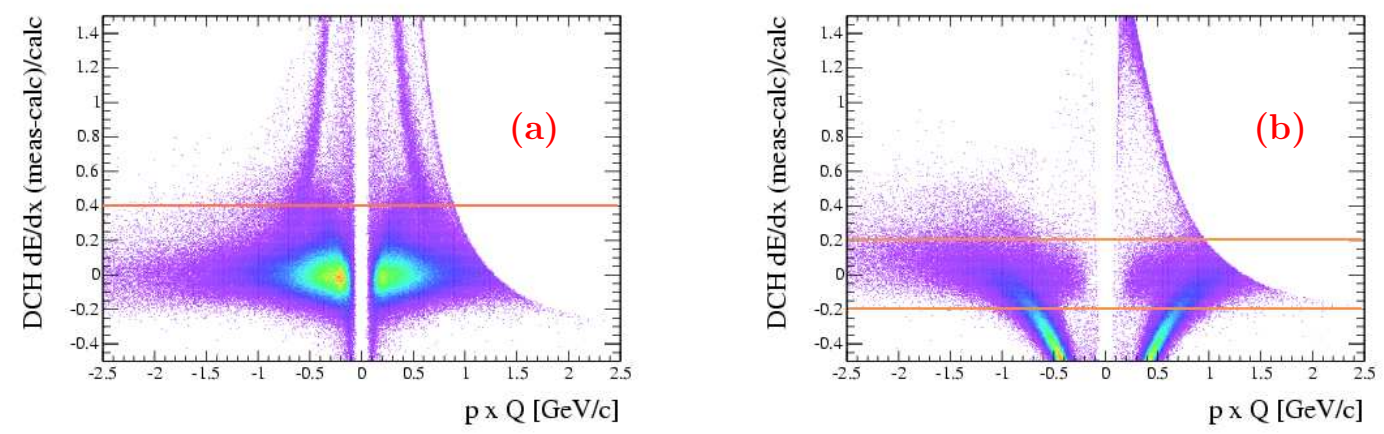

Figure 4.34: (a) The distribution of $C(\pi)$ versus charge-signed momentum for $\left(K_{S}^{0}, p\right)$ vertex-associated tracks after removing vertices with at least one associated baryon; (b) the distribution of $C(K)$ versus charge signed momentum for the same tracks as in (a).

Pions are identified by means of the following criteria: 


$$
C(\pi)<0.4 \text { and } C(K)<-0.2
$$

or

$$
C(\pi)<0.4 \text { and }|C(K)|<0.2
$$

and KVL false,

where "KVL" is the Kaon Very Loose likelihood selector. The relevant regions are indicated by the lines in fig. 4.34.

In addition, a $\left(K_{S}^{0}, p\right)$ vertex rejected because of baryon-association was recovered if the relevant track had $C(\pi)<0.4$ and if the pion Likelihood Tight Selector was satisfied.

In fig. 4.35(a)-(d) the invariant mass distributions obtained when pions identified in this way are combined with the $K_{S}^{0}$ (fig. 4.35(a), (b)) and proton (fig. 4.35(c), (d)) forming a candidate $\left(K_{S}^{0}, p\right)$ vertex are shown; in each figure the upper distribution is for the $K_{S}^{0}$ signal region, and the lower is for the $K_{S}^{0}$ sideband region.

In fig. 4.35(a), the upper $K_{S}^{0} \pi^{+}$mass distribution exhibits a clear $K^{*}(892)^{+}$signal containing $\sim 30,000$ events above background; there is also some small indication of a $K_{2}^{*}(1430)^{+}$signal. The sideband distribution shows no evidence of $K^{*}(892)$, as would be expected. In fig. $4.35(\mathrm{~b})$, the upper $K_{S}^{0} \pi^{-}$distribution exhibits a $\bar{K}^{*}(892)^{-}$signal of $\sim 9,000$ events, with no signal in the $K_{S}^{0}$ sideband distribution. The distributions of figs. 4.35(a) and (b) indicate that more of the selected $K_{S}^{0}$ candidates correspond to $K^{0}$ 's than to $\bar{K}^{0}$ 's at production, and hence that associated production in conjunction with a hyperon contributes significantly to the $K_{S}^{0}$ sample.

The $p \pi^{+}$mass distribution for the $K_{S}^{0}$ signal region (fig. 4.35(c)) may contain some $\Delta(1232)^{++}$, but this is very dependent on assumptions about the the shape of the background. Similarly, there is no obvious $N^{*}$ signal in the $p \pi^{-}$distribution of fig. $4.35(\mathrm{~d})$. There is, however, a large $\Lambda(1115)$ signal in figure fig. 4.35(d), for which the $\Lambda$ region is shown in more detail in fig. 4.36. From this distribution a signal of $\sim 8,700 \Lambda$ 's above background is estimated. These events correspond to an interaction in which a $\Lambda$ and a $K^{0}$ are produced, and the $\Lambda$ decays to $p \pi^{-}$after a very short flight path. This allows the $p$ and the $K_{S}^{0}$ to satisfy the selection criteria for a $\left(K_{S}^{0}, p\right)$ candidate, but when the decay $\pi^{-}$is associated with the vertex, a $\Lambda$ signal appears in the $p \pi^{-}$mass distribution.

In fig. 4.37(a) the SBS $K_{S}^{0} p$ mass distribution is shown for vertices with an associated $\pi^{+}$and no associated baryon or $\bar{p}$. The lower distribution is obtained after removing vertices for which there is a $K_{S}^{0} \pi^{+}$mass combination in the $K^{*}(892)^{+}$region $(0.8<$ $\left.\mathrm{m}\left(K_{S}^{0} \pi^{+}\right)<1.0 \mathrm{GeV} / c^{2}\right)$ of fig. $4.35(\mathrm{a})$. Neither distribution shows evidence of a $\Theta_{5}^{+}$signal. 

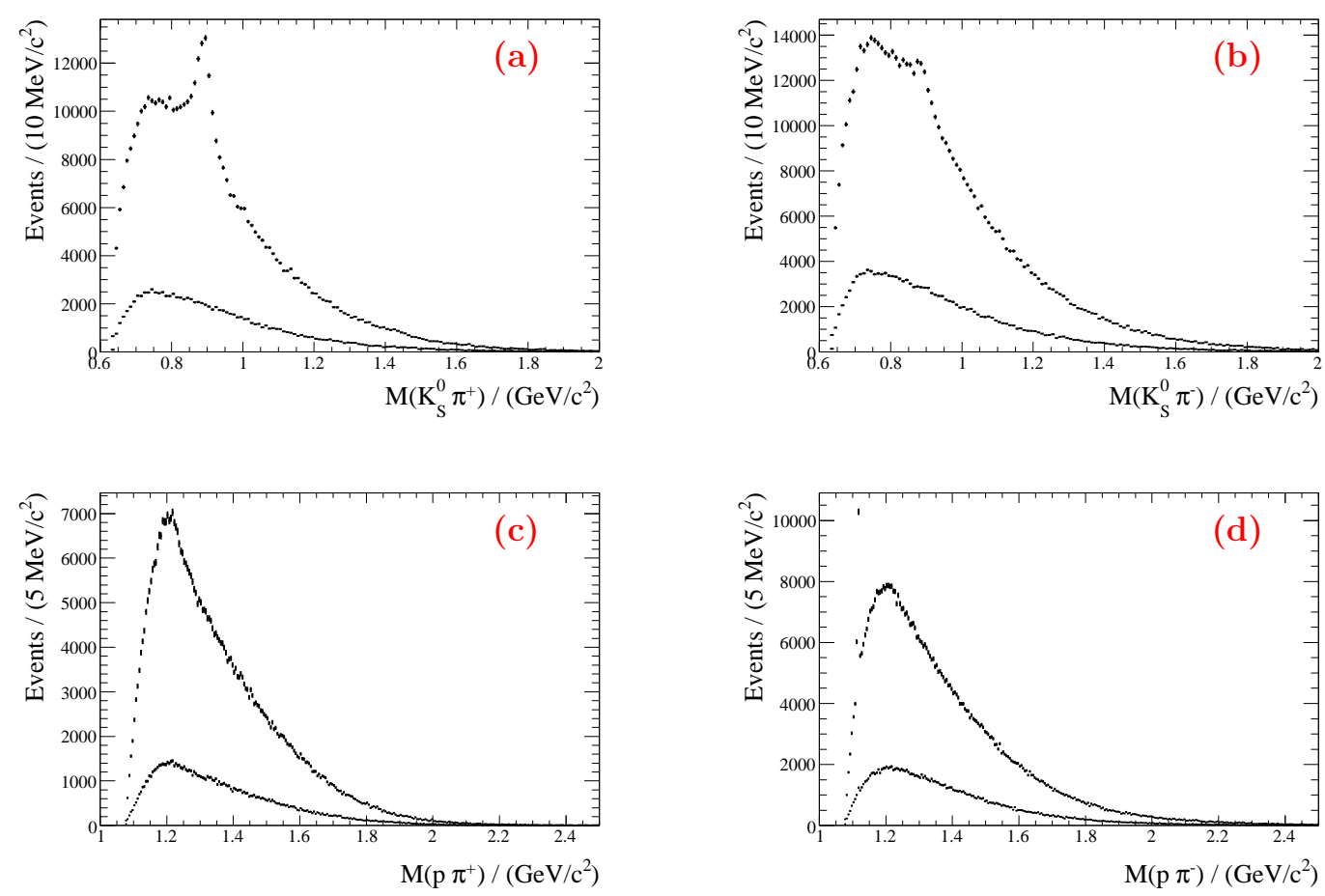

Figure 4.35: Invariant mass distributions for the $p$ or $K_{S}^{0}$ from a candidate $\left(K_{S}^{0}, p\right)$ vertex in combination with an associated $\pi^{ \pm}$. Only vertices with no associated baryon or $\bar{p}$ are used.

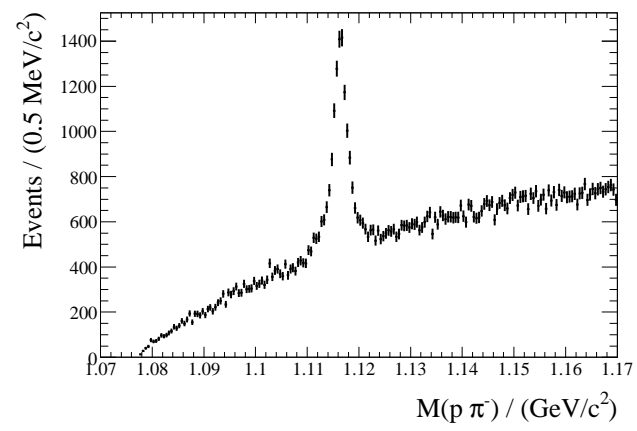

Figure 4.36: The $\Lambda$ signal region of fig. 4.35(d).

Similarly, fig. 4.37(b) shows the SBS $K_{S}^{0} p$ distribution for vertices with an associated $\pi^{-}$. The intermediate distribution is obtained by removing vertices contributing to the $\Lambda$ region of fig. $4.36\left(1.110<\mathrm{M}\left(p \pi^{-}\right)<1.124 \mathrm{GeV} / c^{2}\right)$, and the lowest distribution is obtained by then in addition removing those vertices contributing to the $\bar{K}^{*}(892)^{-}$region of fig. $4.35(\mathrm{~b})$. None of the $K_{S}^{0} p$ mass distributions exhibits a $\Theta_{5}^{+}$signal. 

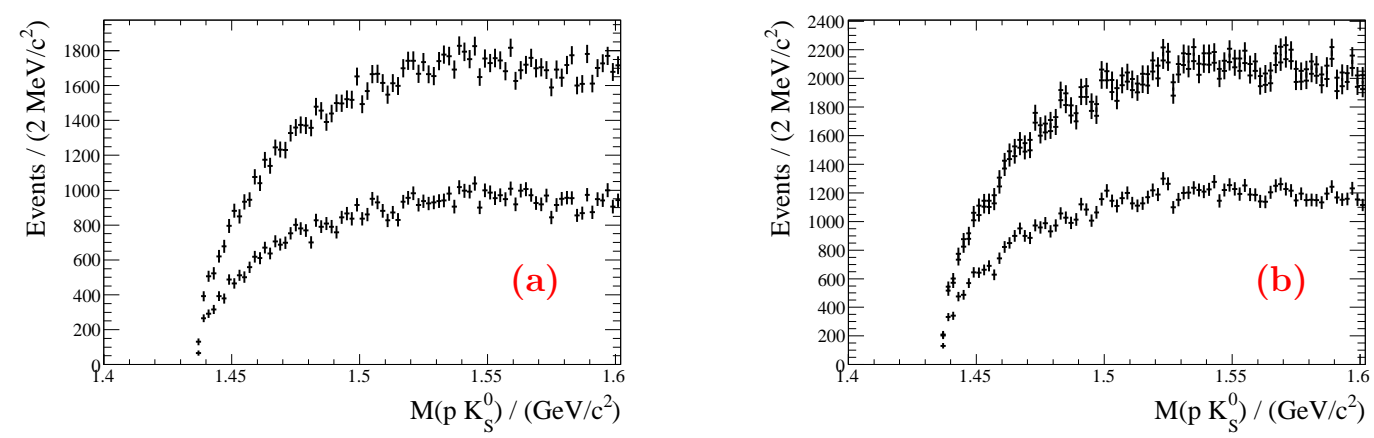

Figure 4.37: (a) The SBS $K_{S}^{0} p$ mass distributions for vertices with an associated $\pi^{+}$and no associated baryon; the lower distribution is obtained by removing candidates which contribute to the $K^{*}(892)^{+}$region of fig. 4.35 (a); (b) the SBS $K_{S}^{0} p$ mass distributions for vertices with an associated $\pi^{-}$and no associated baryon; the intermediate distribution is obtained when the $\Lambda$ region of fig. 4.36 is removed, and the lowest distribution is obtained by then removing the $\bar{K}^{*}(892)^{-}$region of fig. 4.35 (b).

\section{$\left(K_{S}^{0}, p\right)$ Candidates with an Associated $K^{+}$or $K^{-}$}

In this case, a $\left(K_{S}^{0}, p\right)$ vertex-associated track is identified as a kaon by using the following criteria based on fig. 4.34:

$$
|C(K)|<0.2 \quad \text { and } \quad C(\pi)>0.4
$$

or

$$
|C(K)|<0.2 \quad \text { and } \quad C(\pi)<0.4
$$

and KVL true,

where "KVL" is the Kaon Very Loose likelihood selector.

Vertices which have at least one additional track identified as an associated baryon or $\bar{p}$, identified as described in Section 4.6.3, are rejected.

The invariant mass plots corresponding to those of figs. 4.35(a)-(d), but with a pion replaced by a kaon, are shown in figs. $4.38(\mathrm{a})-(\mathrm{d})$. The $K^{-} K_{S}^{0}$ mass distribution (fig. 4.38(b)) seems to accumulate more toward threshold than does the $K^{+} K_{S}^{0}$ distribution of fig. 4.38(a). This might result from there being more $K^{0}$ than $\bar{K}^{0}$ at production, with the effect being due to the presence of the $a_{0}(980)^{-}$just below threshold. The $p K^{+}$mass distribution of fig. 4.38(c) is featureless; however there is a clear $\Lambda(1520)$ signal of $\sim 600$ events in the $p K^{-}$mass distribution of fig. 4.38(d). In each plot of fig. 4.38, the lower distribution 

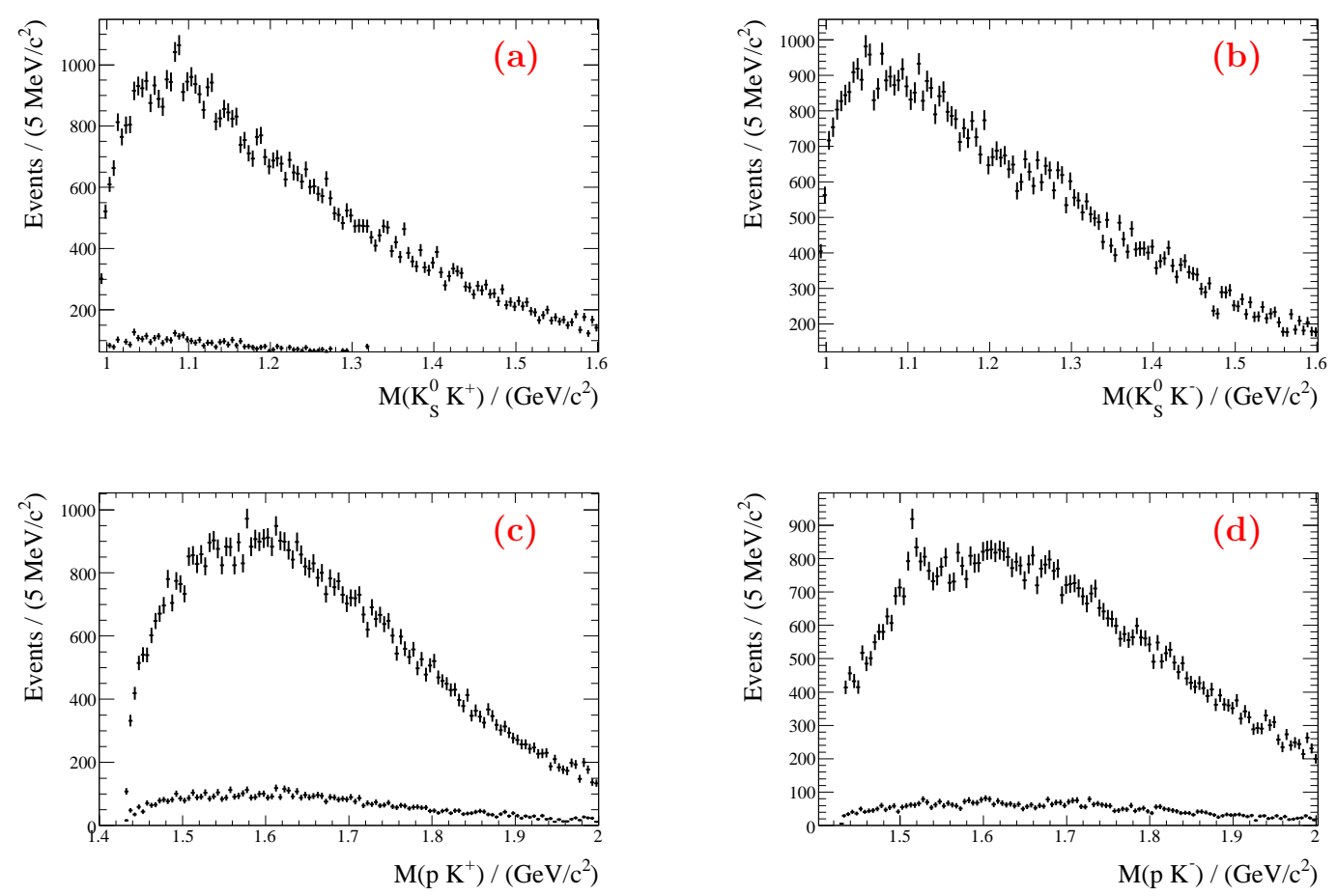

Figure 4.38: Invariant mass distributions for the $p$ or $K_{S}^{0}$ from a candidate $\left(K_{S}^{0}, p\right)$ vertex in combination with an associated $K^{ \pm}$. Only vertices with no associated baryon or $\bar{p}$ are used.

corresponds to the $K_{S}^{0}$ sideband region, and that of fig. 4.38(d) indicates that the $\Lambda(1520)$ signal is indeed associated with $K_{S}^{0}$ production.
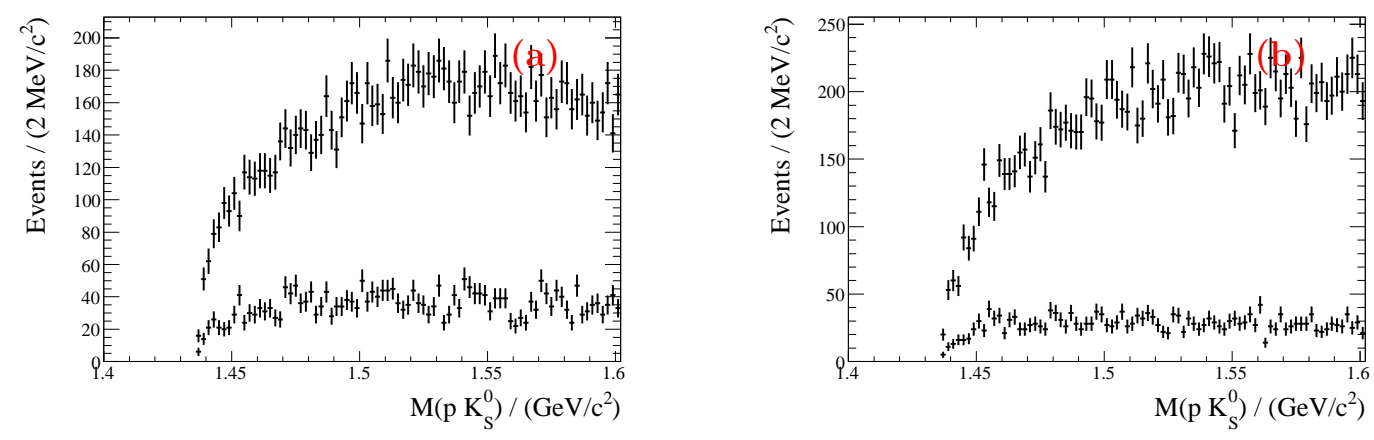

Figure 4.39: The $p K_{S}^{0}$ invariant mass distribution for $\left(K_{S}^{0}, p\right)$ vertices (a) with an associated $K^{+}$, and (b) with an associated $K^{-}$. Only vertices with no associated baryon or $\bar{p}$ are used. The lower distribution in each plot is for the $K_{S}^{0}$ sideband regions.

The $K_{S}^{0} p$ mass distribution corresponding to an associated $K^{+}$(figs. 4.38(a) and (c)) is shown in fig. 4.39(a), while that for an associated $K^{-}$(figs. 4.38(b) and (d)) is shown in fig. 4.39(b). The lower distributions in fig. 4.39 are for the $K_{S}^{0}$ sideband regions. No evidence for the $\Theta_{5}(1540)^{+}$is observed. 


\section{7 $\left(K_{S}^{0}, p\right)$ Electroproduction in Be and Ta}

As discussed previously, the hot spots apparent in fig. 4.21(a) in the horizontal plane are interpreted as resulting from the interactions of off-beam electrons and positrons, primarily in the Be beampipe. Similarly, in figs. 4.21(b)-(d) the hot spots for $x<0$ in the Ta foil regions are interpreted as being due to off-beam electrons. It follows that, if candidate $\left(K_{S}^{0}, p\right)$ vertices are restricted to the geometrical regions of the beampipe and Ta foils near the horizontal plane, the $\left(K_{S}^{0}, p\right)$ system as it results from electroproduction in Be and Ta can be investigated. The regions of interest in the $x-y$ plane (SVT coords.) are defined initially by:

$$
\begin{aligned}
& -3.1<\mathrm{x}<-2.2 \mathrm{~cm} \\
& -0.2<\mathrm{y}<0.7 \mathrm{~cm}
\end{aligned}
$$

and

$$
\begin{aligned}
2.2<\mathrm{x}<3.1 \mathrm{~cm} \\
-0.2<\mathrm{y}<0.5 \mathrm{~cm}
\end{aligned}
$$

and the corresponding $\left(K_{S}^{0}, p\right)$ vertex $z$ distributions are shown in fig. 4.40(a) for $x<0$, and in fig. 4.40(b) for $x>0$ for the interval $|z|<30 \mathrm{~cm}$. The $z<0$ and $z>0$ regions of fig. 4.40(a) are shown in more detail (200 micron bins) in fig. 4.40(c) and fig. 4.40(d), respectively. In fig. 4.40 (a), there is a broad maximum at $z \sim 0$, a very strong spike at $z \sim-8 \mathrm{~cm}$, and a second spike at $z \sim+10 \mathrm{~cm}$ which is about four times weaker. The spikes are due to the Ta foils visible in fig. 4.4 and in fig. 4.21(c) and (d) as the hot spots for $x<0$. In fig. 4.40(b) the height of the central maximum is less than $5 \%$ fig 4.40 (a), and the Ta spikes are almost non-existent. The structure beyond the Ta spikes of fig. 4.40(a) is shown in detail in fig. 4.40(c) for $z<0$ and fig. 4.40(d) for $z>0$. These regions are also shown in the elevation drawing of the beampipe region in fig. 4.41 (note that here $z$ increases from right to left, whereas in fig. $4.40 z$ increases from left to right). In fig. 4.40(c), the structure near $z=-14 \mathrm{~cm}$ is due to the cooling water inlets drawn in fig. 4.41 and visible in fig. 4.4 ; that at $z \sim-18 \mathrm{~cm}$ is due to the stainless steel bellows at which the beampipe is welded to the vacuum chamber (see figs. 4.4 and 4.41); and the decrease at $z \sim-20 \mathrm{~cm}$ and the shape of the subsequent increase are due to the variation in thickness of the vacuum chamber wall visible in fig. 4.41. There are far fewer events in the distribution of fig. $4.40(\mathrm{~d})$, but the bump at $z \sim+18 \mathrm{~cm}$ is probably due to the forward bellows, as drawn in fig. 4.41 .

These structural details again provide convincing evidence that interactions in the material of the detector are indeed being selected. 

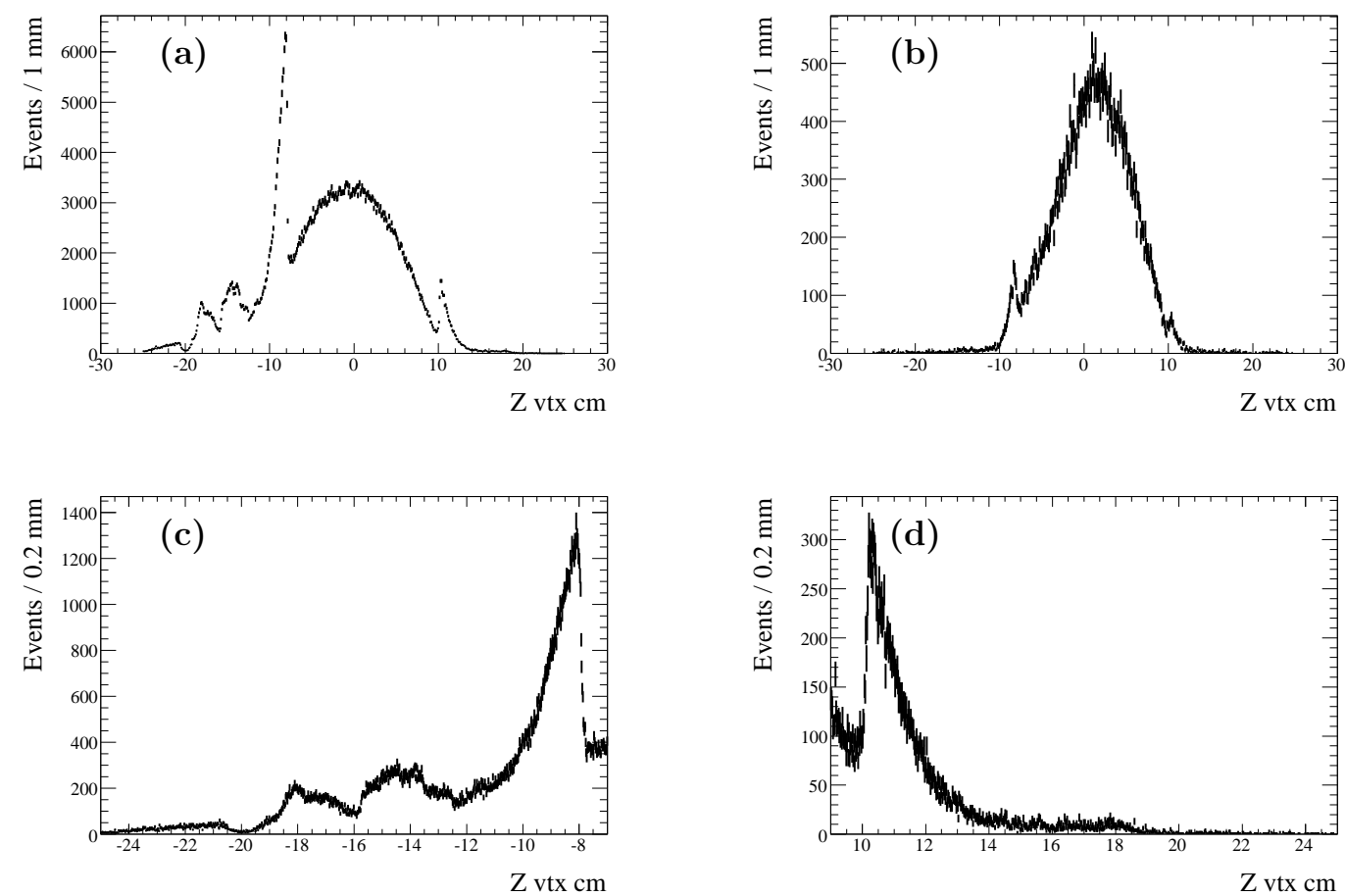

Figure 4.40: (a) The $z$ distribution of $\left(K_{S}^{0}, p\right)$ vertices in the $x<0$ hot spot of fig. 4.21(a); (b) the same for $x>0$; (c) a closer look at the region $-25<z<-7 \mathrm{~cm}$ for $x<0$, and (d) for $9 \leq z \leq 25 \mathrm{~cm}$ and $x<0$.

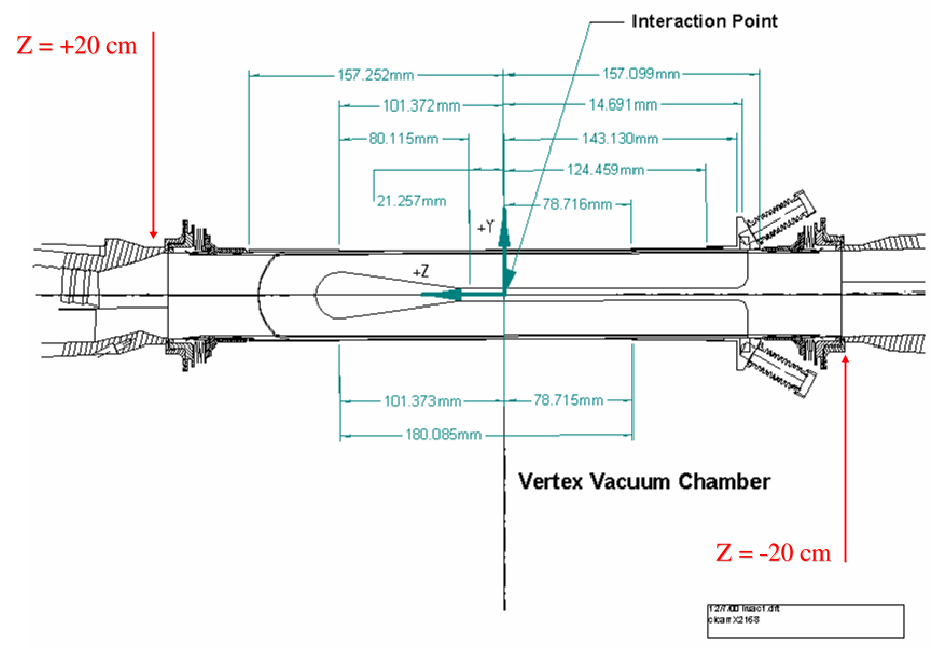

Figure 4.41: An elevation drawing of the beampipe and adjacent vacuum chamber.

Next Be and Ta regions are defined by:

$$
\begin{array}{rll}
-7.7 & <z<10.0 \mathrm{~cm} \quad(\text { Be } x<0 \text { and } x>0) \\
-10.5 & <z<-7.7 \mathrm{~cm} \quad \text { ( Ta backward, } x<0 \text { only) } \\
10.0 & <z<12.5 \mathrm{~cm} \quad \text { (Ta forward, } x<0 \text { only) }
\end{array}
$$


Finally, within each $z$ region the range of radius is restricted to

$$
\begin{aligned}
2.35 & <r<3.0 \mathrm{~cm} \quad(\text { Be })[2.498<r<2.785 \mathrm{~cm} \text { nominal }] \\
2.7 & <r<3.0 \mathrm{~cm} \quad(\text { Ta })[r(\text { center })=2.854 \mathrm{~cm}, \text { Ta thickness } \sim 150 \mu \mathrm{m}] .
\end{aligned}
$$

In the following, only the results for electroproduction of the $\left(K_{S}^{0}, p\right)$ low mass region in Be are discussed. Distributions for the higher mass region are collected in Appendix B, section B.2. The results for he $e^{+}$interactions in Be, and $e^{-}$interactions in Ta are very similar to those for $e^{-}$in Be, but involve many fewer events. The relevant distributions are collected in Appendices $\mathrm{C}$ and D, respectively.

The $e^{-}$, and some of the $e^{+}$results for the interactions in the Be beampipe are now discussed. (Note that the beampipe is not composed of Be only; the other materials contributing to the structure are listed in fig. 4.5).
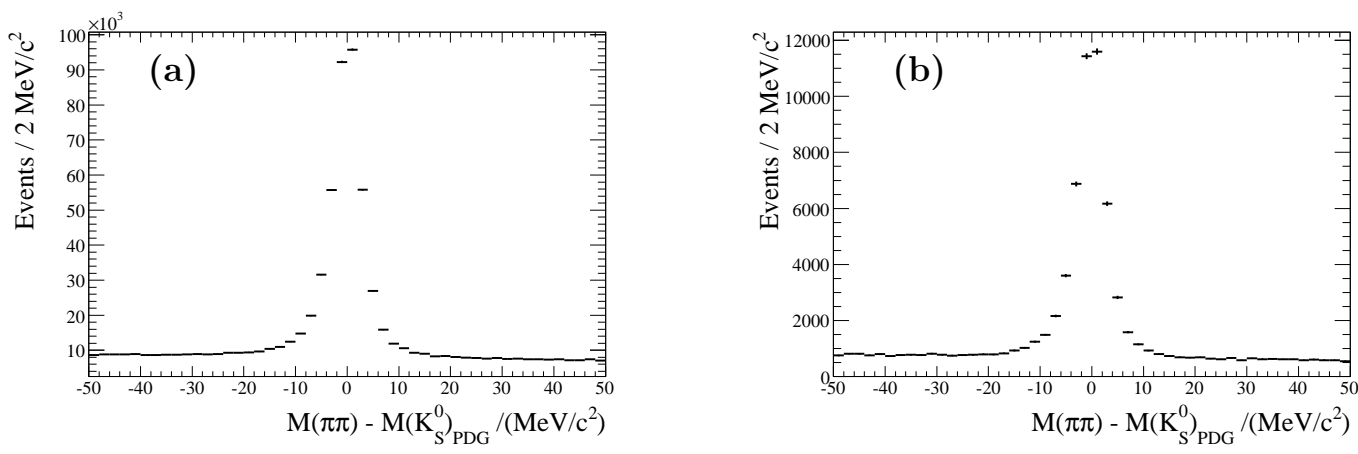

Figure 4.42: The distributions of $\pi^{+} \pi^{-}$invariant mass relative to the $K_{S}^{0}$ PDG value for the Be target regions defined in the text (a) for $x<0\left(e^{-}\right)$, and (b) for $x>0\left(e^{+}\right)$.
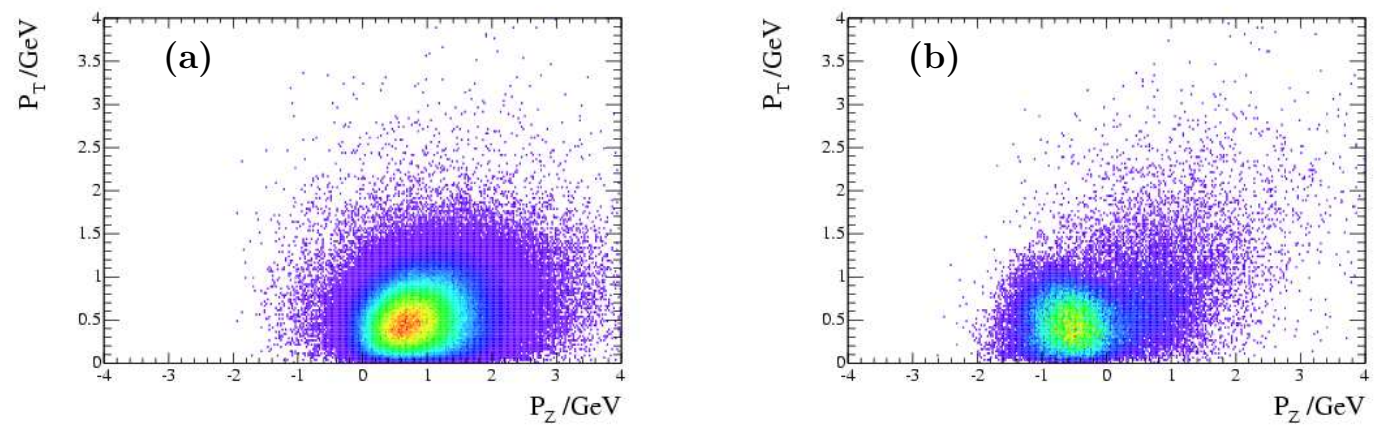

Figure 4.43: The scatterplots of transverse $\left(P_{T}\right)$ and longitudinal $\left(P_{Z}\right)$ momentum for the $\left(K_{S}^{0}, p\right)$ candidates in the Be target regions, (a) for $x<0\left(e^{-}\right)$, and (b) for $x>0\left(e^{+}\right)$.

In figs. 4.42(a) and (b) the $\pi^{+} \pi^{-}$mass distributions relative to the $K_{S}^{0}$ PDG value are shown for candidates from the Be regions with $x<0$ and $x>0$ respectively. Clear $K_{S}^{0}$ signals are observed, with $\mathrm{S} / \mathrm{B}$ values $\sim 5: 1$. As a check of the association of the 
$x<0$ Be hot spot with off-beam electrons and of the $x>0$ Be hot spot with off-beam positrons, the scatterplots of $P_{T}$ versus $P_{Z}$ for these regions are shown in figs. 4.43(a) and (b) respectively. Here

$$
\vec{P}=\vec{p}\left(K_{S}^{0}\right)+\vec{p}(\text { proton })
$$

is the lab. three-momentum sum for the $\left(K_{S}^{0}, p\right)$ system, and $P_{T}$ and $P_{Z}$ are its transverse and longitudinal components. In fig. 4.43(a) the distribution follows the direction of the electron beam, while in fig. 4.43(b) the main part of the distribution follows the direction of the positron beam. The correlations are not very strong since the interactions are due to the emitted virtual photons, not to the beam particles directly, and in addition a baryonic system produced in fixed target collisions is being investigated. Nevertheless, they are present, and become more pronounced with the association of other tracks with the $\left(K_{S}^{0}\right.$, $p)$ vertices (see below).
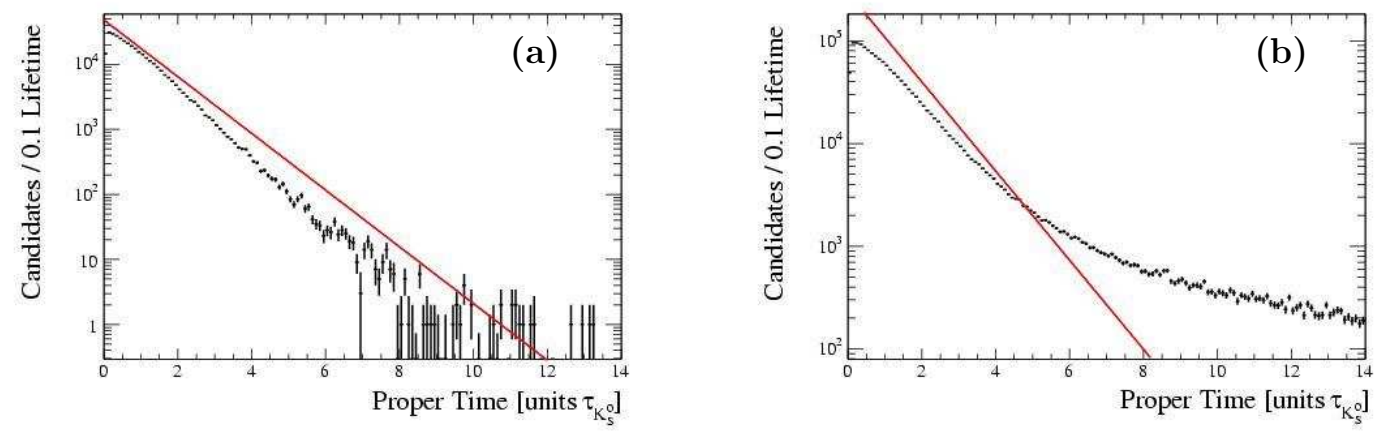

Figure 4.44: (a) The distribution in proper time (units $\tau_{K_{S}^{0}}$ ) for $K_{S}^{0}$ candidates from $e^{-} \mathrm{Be}$ interactions; the line shows the ideal slope; (b) the same distribution for $K_{S}^{0}$ candidates from all $\left(K_{S}^{0}, p\right)$ vertices. For both distributions the $K_{S}^{0}$ sideband region contributions have been subtracted.

The dependence of the distributions of fig. 4.43 on run period is discussed in Appendix B, section B.1. For each run period the observed $P_{T}-P_{Z}$ behavior is very similar to that of fig. 4.43. In particular the weak cluster for $P_{Z} \sim 1-2 \mathrm{GeV} / c$ in fig. $4.43(\mathrm{~b})$ is present for all Run Periods. This cluster is interpreted as being due to the secondary interactions of hadrons produced in $e^{+} e^{-}$collisions, with the positive $P_{Z}$ resulting from the forward boost from the c.m. frame to the lab. frame because of the asymmetry of the PEP-II collider.

In selecting $\left(K_{S}^{0}, p\right)$ candidates resulting from $e^{+}$Be collisions it is required that $P_{Z}$ be less that $0.5 \mathrm{GeV} / c$ in order to remove most of these events. The corresponding events from the $e^{-}$Be sample cannot be removed, but they should be similar in number to those in fig. 4.43(b) and so constitute a background of $\sim 1 \%$.

As a further check on the quality of the $e^{-}$Be sample, the SBS $K_{S}^{0}$ proper time 
distribution in units of $\tau_{K_{S}^{0}}$ is shown in fig. 4.44(a); this is calculated for each event as

$$
\frac{\tau}{\tau_{K_{S}^{0}}}=\frac{m c^{2}}{p c \cdot c \tau_{K_{S}^{0}}} \times S F L
$$

where $m$ and $\tau_{K_{S}^{0}}$ are the PDG mass and lifetime values for the $K_{S}^{0}, p$ is the lab. momentum and $S F L$ is the signed flight length of the $K_{S}^{0}$ candidate.
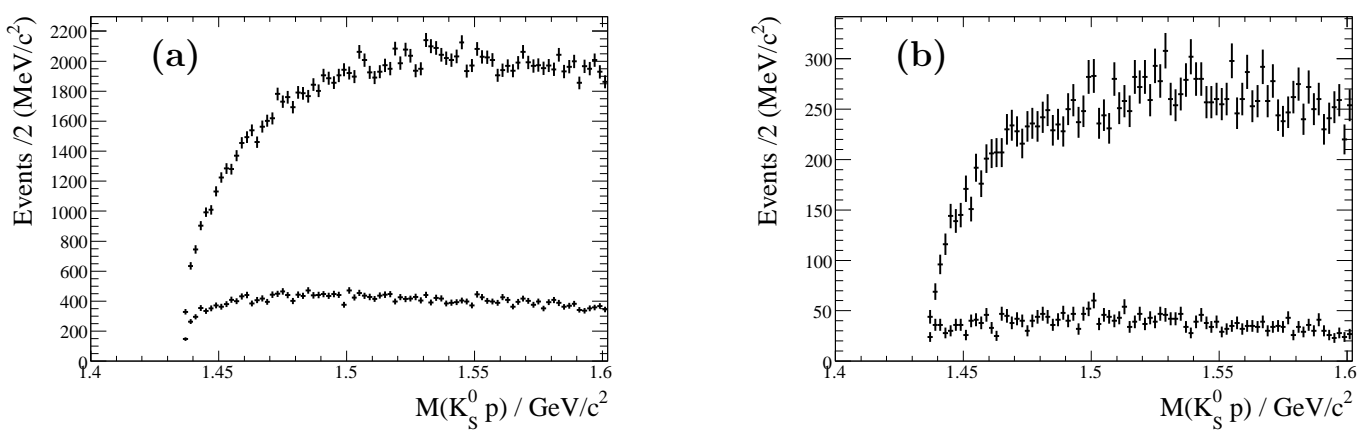

Figure 4.45: The inclusive $K_{S}^{0} p$ mass distributions for electroproduction in Be (a) for $\mathrm{x}<0$ $\left(e^{-}\right)$and $(\mathrm{b})$ for $\mathrm{x}>0\left(e^{+}\right)$; the lower distributions are for the $K_{S}^{0}$ sideband regions.

The line indicates the ideal $K_{S}^{0}$ slope corresponding to the distribution $\sim e^{-x}$ with $x=\tau / \tau_{K_{S}^{0}}$. For $\sim 0.5-1.5$ lifetimes the data seem to have the correct slope. There are losses at lower values, and there is a significant drop below 0.1 lifetime; these are probably due to the requirement $S F L>0.2 \mathrm{~cm}$ and track reconstruction inefficiency at low transverse momentum. The losses beyond 1.5 lifetimes are the result of the finite tracking region (radius less than $\sim 50 \mathrm{~cm}$ for the $K_{S}^{0}$ decay point) and the cylindrical nature of the tracking devices (e.g. tracks approximately parallel to the DCH wires cannot be found). The observed behavior thus seems reasonable.

The corresponding behavior for all $\left(K_{S}^{0}, p\right)$ candidates is shown in fig. 4.44(b). At short lifetimes, the behavior is similar to that in fig. 4.44(a), but beyond $\sim 2$ lifetimes the slope of the distribution becomes smaller than the expected slope, indicated by the line. Since $K_{S}^{0} \mathrm{~S} / \mathrm{B}$ improves greatly with flight path (fig. 4.19) and since the $\left(K_{S}^{0}, p\right)$ vertices reproduce the detector geometry very well, it does not seem that the lifetime behavior should be attributed to spurious $K_{S}^{0}$-proton association, especially since there is no such effect in fig. 4.44(a). A major difference between the data samples is that for the $e^{-} \mathrm{Be}$ sample the neutral kaons encounter very little material along their flight paths prior to their decay. This is not true for many parts of the detector e.g. from the Ta foils. Since the selection criteria are the same for all candidates, the behavior in fig. 4.44(b) is interpreted as being due to $K_{L}^{0}-K_{S}^{0}$ regeneration. The regenerated $K_{S}^{0}$ would still point back well to the $\left(K_{L}^{0}, p\right)$ production point and have the same momentum as the original $K_{L}^{0}$, since regeneration is essentially a forward scattering process. Consequently, the long-lived $K_{S}^{0}$ candidates were not discarded in the analysis of the complete $\left(K_{S}^{0}, p\right)$ candidate sample 
presented in Section 4.6.

In the study of the $\left(K_{S}^{0}, p\right)$ system produced in the Be beampipe, the same procedures used in Section 4.6 for the general production in the material of the BABAR detector are followed.

\subsubsection{The Inclusive $K_{S}^{0} p$ Invariant Mass Distribution}

The $K_{S}^{0} p$ invariant mass distributions are shown in figs. 4.45(a) and (b) for $x<0\left(e^{-}\right)$ and $x>0\left(e^{+}\right)$respectively; the lower distributions are for the $K_{S}^{0}$ sideband regions. No $\Theta_{5}(1540)^{+}$signal is observed.
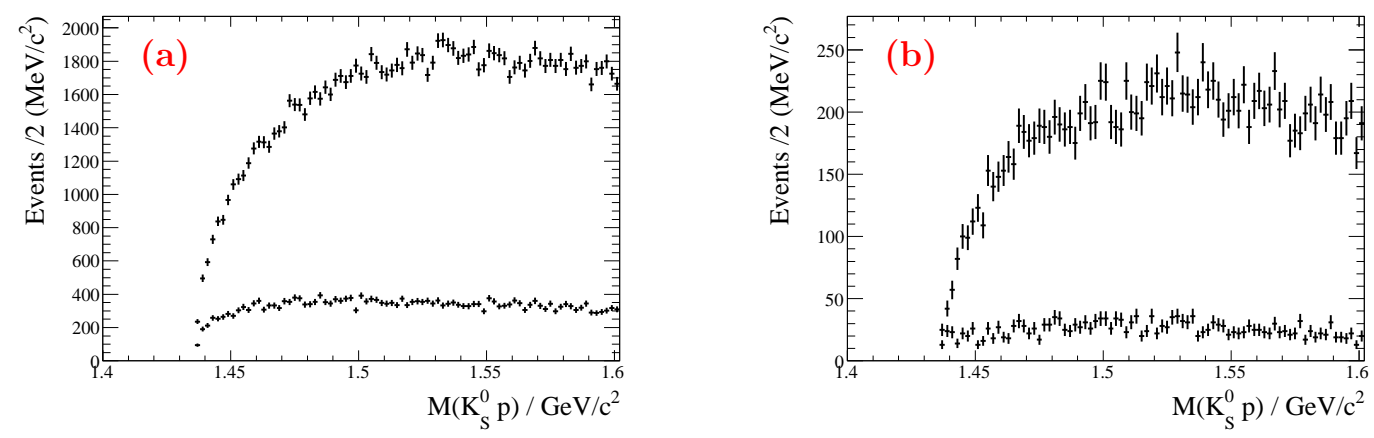

Figure 4.46: The $K_{S}^{0} p$ mass distributions for $\left(K_{S}^{0}, p\right)$ vertices with at least one associated track, for regions $(\mathrm{a}) x<0\left(e^{-}\right)$, and (b) $x>0\left(e^{+}\right)$; the lower distributions are for the $K_{S}^{0}$ sideband regions.

\subsubsection{The Inclusion of Additional Charged Tracks}

Additional charged tracks are associated with candidate $\left(K_{S}^{0}, p\right)$ vertices as described in Section 4.6.2. When it is required that there be at least one such track, the $K_{S}^{0} p$ invariant mass distributions of figs. 4.46(a) and (b) are obtained for the $e^{-}$and $e^{+}$samples, respectively. The lower distribution in each plot is for the $K_{S}^{0}$ sideband regions. No $\Theta_{5}(1540)$ signal can be seen.

\subsubsection{The Identification of Associated Charged Tracks}

The particle identification procedures of Section 6.3 are followed here, but in addition associated electron tracks are identified, as discussed below.

\section{Rejection of $\left(K_{S}^{0}, p\right)$ Candidates with an Associated Baryon}

Candidate $\left(K_{S}^{0}, p\right)$ vertices with at least one associated track considered to be a baryon or $\bar{p}$ are rejected for the same reasons, and following the same procedures, as those described in Section 4.6.3. The $K_{S}^{0} p$ mass distribution for surviving candidates is shown in fig. 4.47(a) 

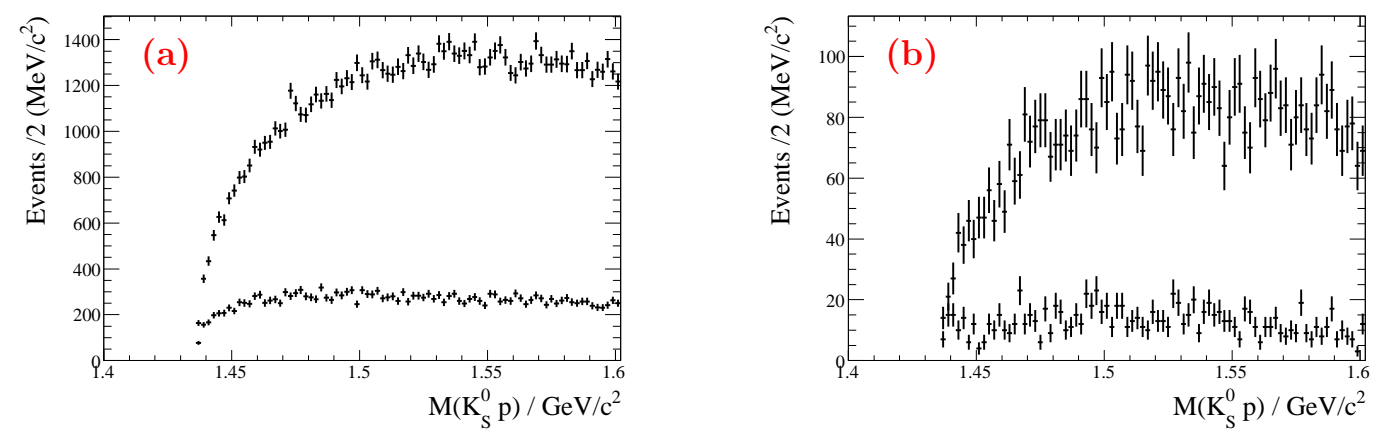

Figure 4.47: The $K_{S}^{0} p$ mass distribution after removing vertices with at least one associated baryon or $\bar{p}$, for regions (a) $x<0\left(e^{-}\right)$, and (b) $x>0\left(e^{+}\right)$.

for electron events, and fig. 4.47(b) for positron events. As usual, the lower distributions are for the $K_{S}^{0}$ sidebands, and as usual there is no $\Theta_{5}(1540)$ signal to be seen.

In the following, only the $e^{-}$(i.e. $\left.x<0\right)$ results are discussed. The $e^{+}$results are very similar, but suffer from significantly lower statistics (see Appendix C).

\section{$\left(K_{S}^{0}, p\right)$ Candidates with an Associated $\pi^{+}$or $\pi^{-}$}
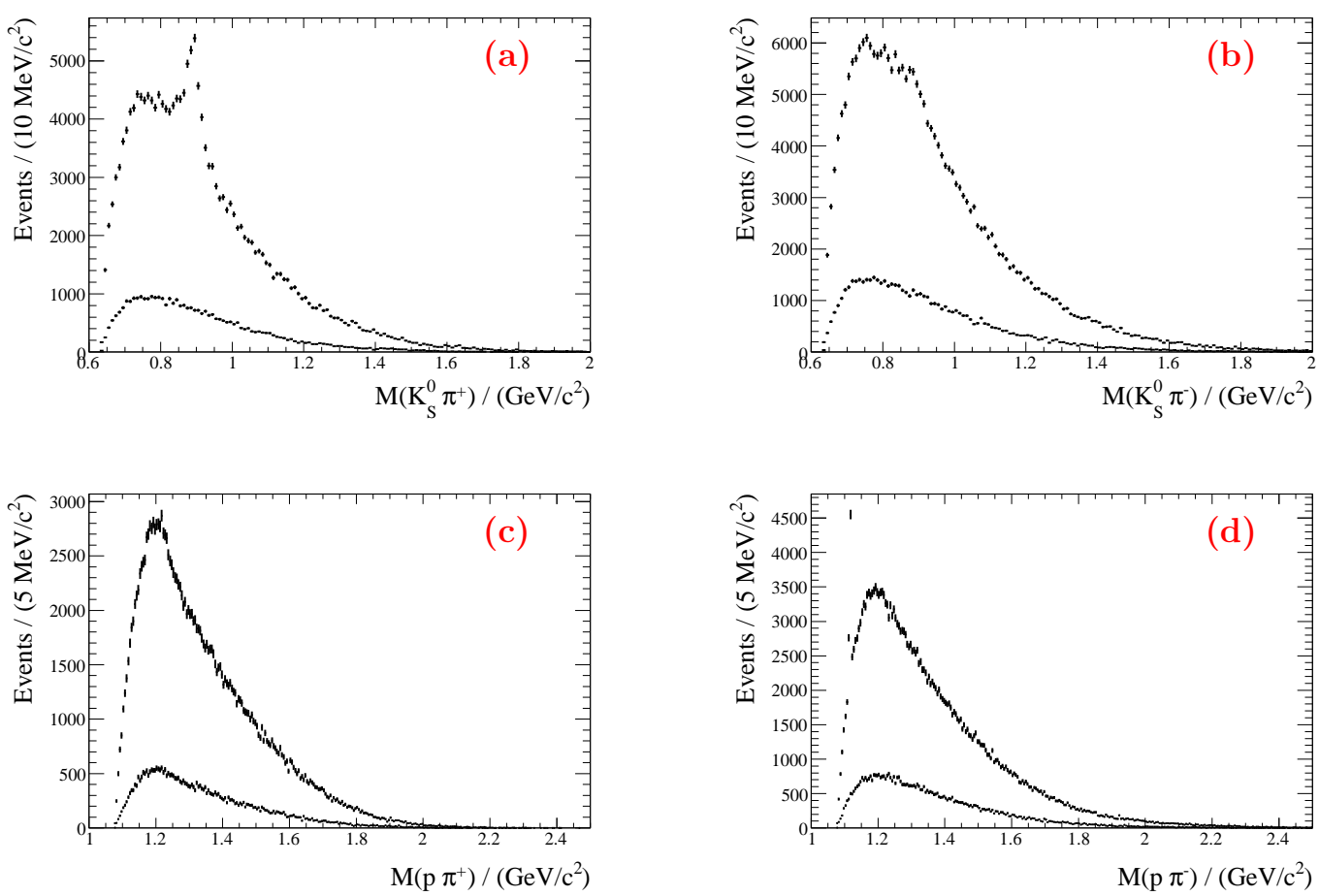

Figure 4.48: Invariant mass distributions for the $p$ or $K_{S}^{0}$ from a candidate $\left(K_{S}^{0}, p\right)$ vertex in combination with an associated $\pi^{ \pm}$. Only vertices with no associated baryon or $\bar{p}$ are used.

In figs. 4.48(a)-(d) the invariant mass distributions obtained when identified charged 

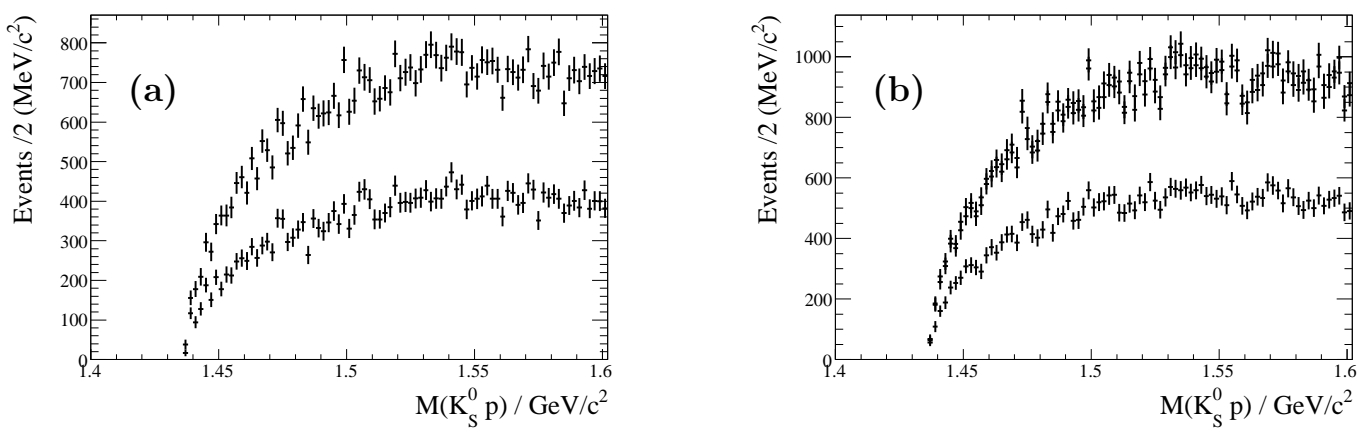

Figure 4.49: (a) The SBS $K_{S}^{0} p$ mass distributions for vertices with an associated $\pi^{+}$and no associated baryon; the lower distribution is obtained by removing candidates which contribute to the $K^{*}(892)^{+}$region of fig. 4.48 (a); (b) the SBS $K_{S}^{0} p$ mass distributions for vertices with an associated $\pi^{-}$and no associated baryon; the intermediate distribution is obtained when the $\Lambda$ region (cf. fig. 4.36) is removed, and the lowest distribution is obtained by then removing the $\bar{K}^{*}(892)^{-}$region of fig. $4.48(\mathrm{~b})$.

pions are combined with the $K_{S}^{0}$ (figs. 4.48(a) and (b)) and proton (figs. 4.48(c) and (d)) forming a candidate $\left(K_{S}^{0}, p\right)$ vertex are shown; in each figure the upper distribution is for the $K_{S}^{0}$ signal region, while the lower is for the $K_{S}^{0}$ sidebands. These distributions exhibit the same features as those of fig. 4.35, but with fewer entries. The $K^{*}(892)^{+}$signal contains $\sim 10,000$ events above background, while there are $\sim 3,500 \bar{K}^{*}(892)^{-}$events and $\sim 2,500$ events in the $\Lambda(1115)$ signal in fig. $4.48(\mathrm{~d})$. The SBS $K_{S}^{0} p$ invariant mass distributions of fig. 4.49 exactly parallel those of fig. 4.37, and lead to the same conclusion - no evidence for $\Theta_{5}(1540)$.

\section{$\left(K_{S}^{0}, p\right)$ Candidates with an Associated $K^{+}$or $K^{-}$}

In figs. 4.50(a)-(d) the invariant mass distributions obtained when identified charged kaons are combined with the $K_{S}^{0}$ (figs. 4.50(a) and (b)) and proton (figs. 4.50(c) and (d)) forming a candidate $\left(K_{S}^{0}, p\right)$ vertex are shown; in each figure the upper distribution is for the $K_{S}^{0}$ signal region, while the lower is for the $K_{S}^{0}$ sidebands. These distributions exhibit the same features as those of fig. 4.38, but with fewer entries. The $\Lambda(1520)$ signal in fig. $4.50(\mathrm{~d})$ is estimated to contain $\sim 350$ events above background. The SBS $K_{S}^{0} p$ invariant mass distributions of fig. 4.51 exactly parallel those of fig. 4.39, and once more there is no evidence for $\Theta_{5}(1540)$ production.

For the $\left(K_{S}^{0}, p\right)$ candidates with an associated $K^{-}$the lab. three-momentum, $\vec{P}$, is formed as before, but now the $K^{-}$three-vector is added also. The scatterplots of $P_{T}$ versus $P_{Z}$ analogous to those of fig. 4.43 are shown in figs. 4.52(a) and (b), and it is seen that the inclusion of the $K^{-}$vector moves the electron-side concentration more in the direction of the electron beam, and the positron-side concentration more in the direction of the positron beam, further validating the association of the negative and positive $x$-regions with $e^{-}$and $e^{+}$off-beam interactions. 

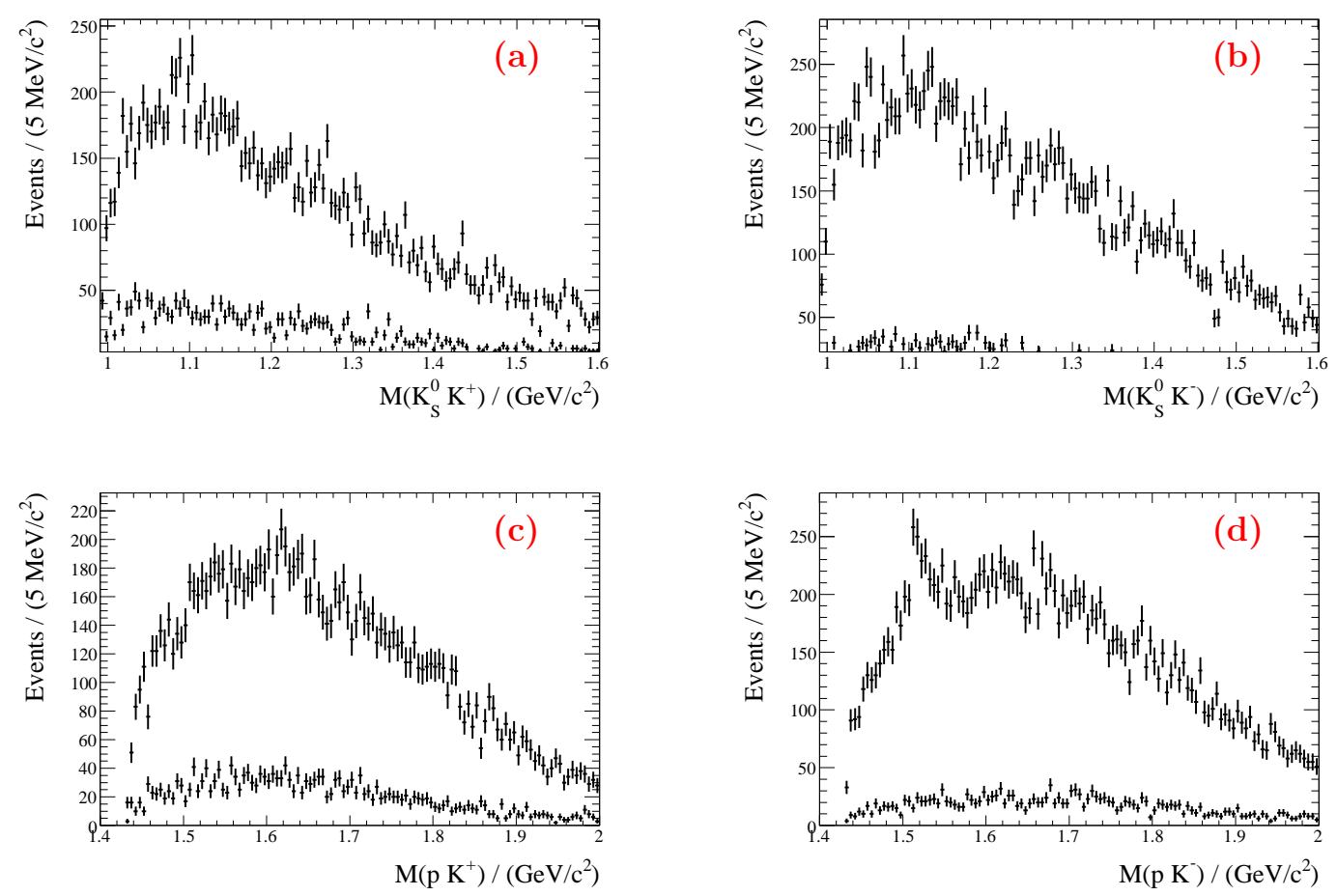

Figure 4.50: Invariant mass distributions for the $p$ or $K_{S}^{0}$ from a candidate $\left(K_{S}^{0}, p\right)$ vertex in combination with an associated $K^{ \pm}$. Only vertices with no associated baryon or $\bar{p}$ are used.
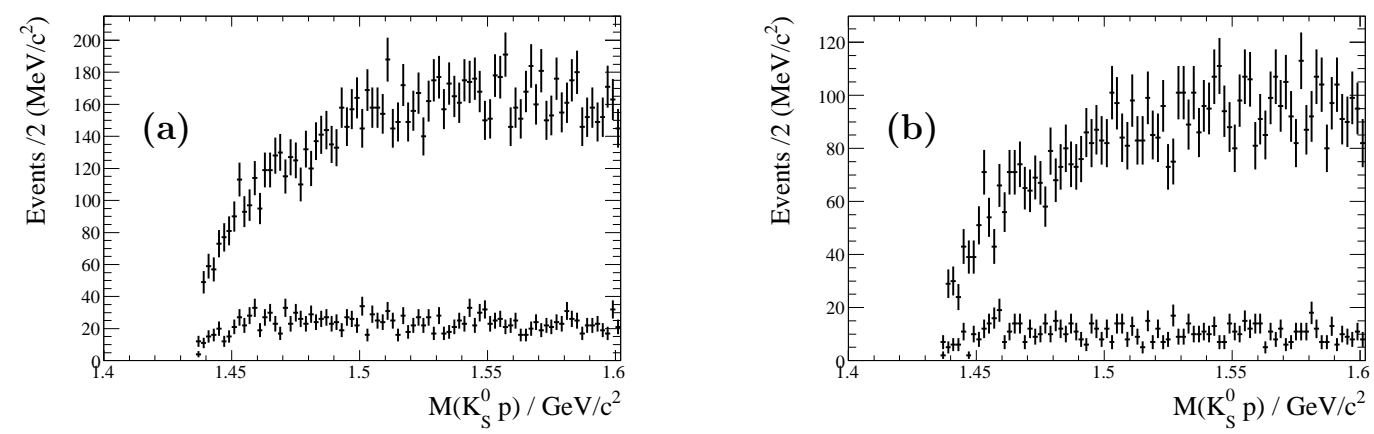

Figure 4.51: The $p K_{S}^{0}$ invariant mass distribution for $\left(K_{S}^{0}, p\right)$ vertices (a) with an associated $K^{+}$, and (b) with an associated $K^{-}$. Only vertices with no associated baryon or $\bar{p}$ are used. The lower distribution in each plot is for the $K_{S}^{0}$ sideband regions.

The $K_{S}^{0} p K^{-}$invariant mass distributions for these events are shown in fig. 4.53. The $e^{-}$Be sample of fig. 4.53(a) contains $\sim 19,000$ candidates, whereas that of fig. 4.53(b) contains only $\sim 600$; both distributions peak at $\sim 2.3-2.4 \mathrm{GeV} / c^{2}$, but reveal no significant structure. In the CLAS analysis of the reaction $\gamma p \rightarrow K^{+} K^{-} \pi^{+} n$ [41] when a leading $\pi^{+}$ was selected, it was observed that the $\Theta_{5}^{+}$structure in the $K^{+} n$ system was correlated with a narrow structure in the $K^{+} n K^{-}$mass distribution near $2.4 \mathrm{GeV} / c^{2}$. It was suggested that this might be due to the production of an $N^{*}$ resonance, which subsequently decayed 

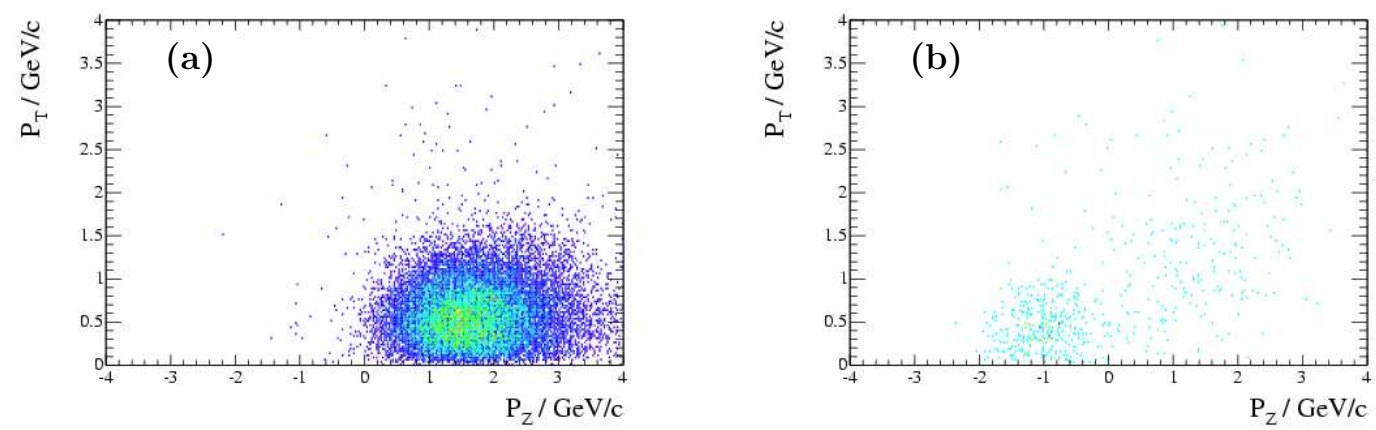

Figure 4.52: The scatterplots of transverse $\left(P_{T}\right)$ and longitudinal $\left(P_{Z}\right)$ three-momentum for the $\left(K_{S}^{0}, p, K^{-}\right)$vertices in the Be target regions, (a) for $\mathrm{x}<0$, and (b) for $\mathrm{x}>0$.
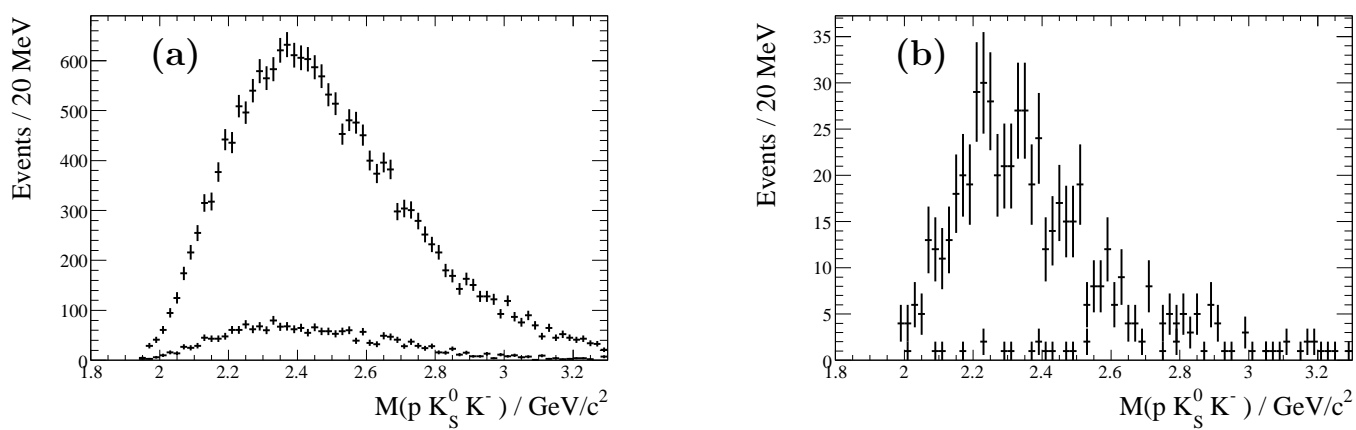

Figure 4.53: (a) The $K_{S}^{0} p K^{-}$invariant mass distribution for $\left(K_{S}^{0}, p\right)$ candidates with an associated $K^{-}$from the $e^{-}$Be interaction sample $(\mathrm{x}<0)$; (b) the same for the $e^{+} \mathrm{Be}$ sample $(\mathrm{x}>0)$. The lower distribution in each plot is for the $K_{S}^{0}$ sidebands.

to $\Theta_{5}^{+} K^{-}$. For this reason the $K_{S}^{0} p$ mass distributions corresponding to the $K_{S}^{0} p K^{-}$ mass intervals $2.2 \pm 0.1,2.4 \pm 0.1,2.6 \pm 0.1 \mathrm{GeV} / c^{2}$ of fig. $4.53(\mathrm{a})$ are shown in fig. 4.54(a), fig. 4.54(b) and fig. 4.54(c) respectively. In fig. 4.54(b) there are two small, but intriguing, peaks in the $1.5-1.6 \mathrm{GeV} / c^{2}$ mass region, but these are quite consistent with statistical fluctuation.
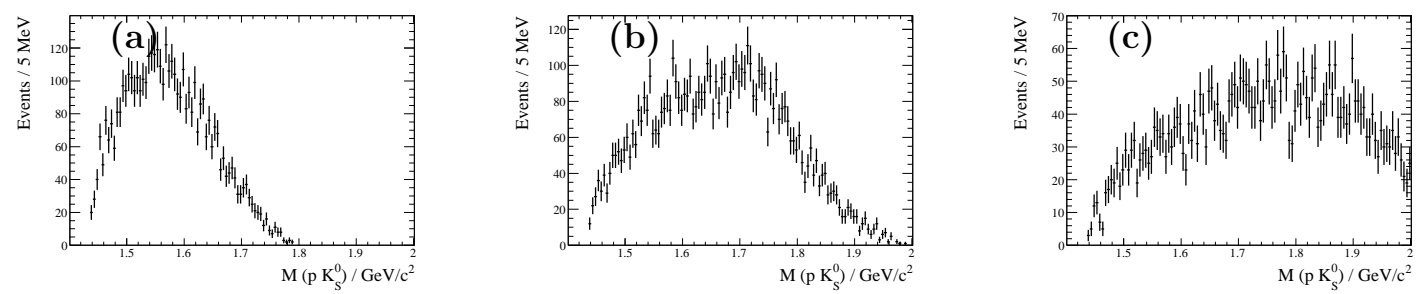

Figure 4.54: The $K_{S}^{0} p$ mass distributions corresponding to the regions (a) $2.2 \pm 0.1$, (b) $2.4 \pm 0.1$, (c) $2.6 \pm 0.1 \mathrm{GeV} / c^{2}$ of fig. $4.53(\mathrm{a})$.

In figs. 4.50(b) and (d) the mass distribution does not drop rapidly to zero as threshold 
is approached. Since the mass projections contain the multiplicative two-body phase space factor, q (the magnitude of the three-momentum in the two-body rest frame, which goes to zero at threshold), this implies that the matrix element squared integrated over the rest of the event phase space must be large near threshold. In order to test this conjecture, individual events are given weight $\mathrm{q}_{0} / \mathrm{q}$, where $\mathrm{q}_{0}$ is $\mathrm{q}$ evaluated at a fixed mass $\sim 200 \mathrm{MeV} / \mathrm{c}^{2}$ above threshold, and the SBS distributions of figs. 4.50(b) and (d) replotted using these event weights. The results are shown in figs. 4.55(a) and (b), and both distributions increase rapidly as threshold is approached. This suggests that the $K_{S}^{0} K^{-}$distribution is strongly influenced by the $a_{0}(980)$ state just below threshold, and that the $p K^{-}$distribution is similarly influenced by the $\Lambda(1405)$. The corresponding results for the distributions of figs. $4.38(\mathrm{~b})$ and (d), which use $\left(K_{S}^{0}, p\right)$ vertices from all detector material, are shown in figs. 4.55(c) and (d), and the same effects are seen.
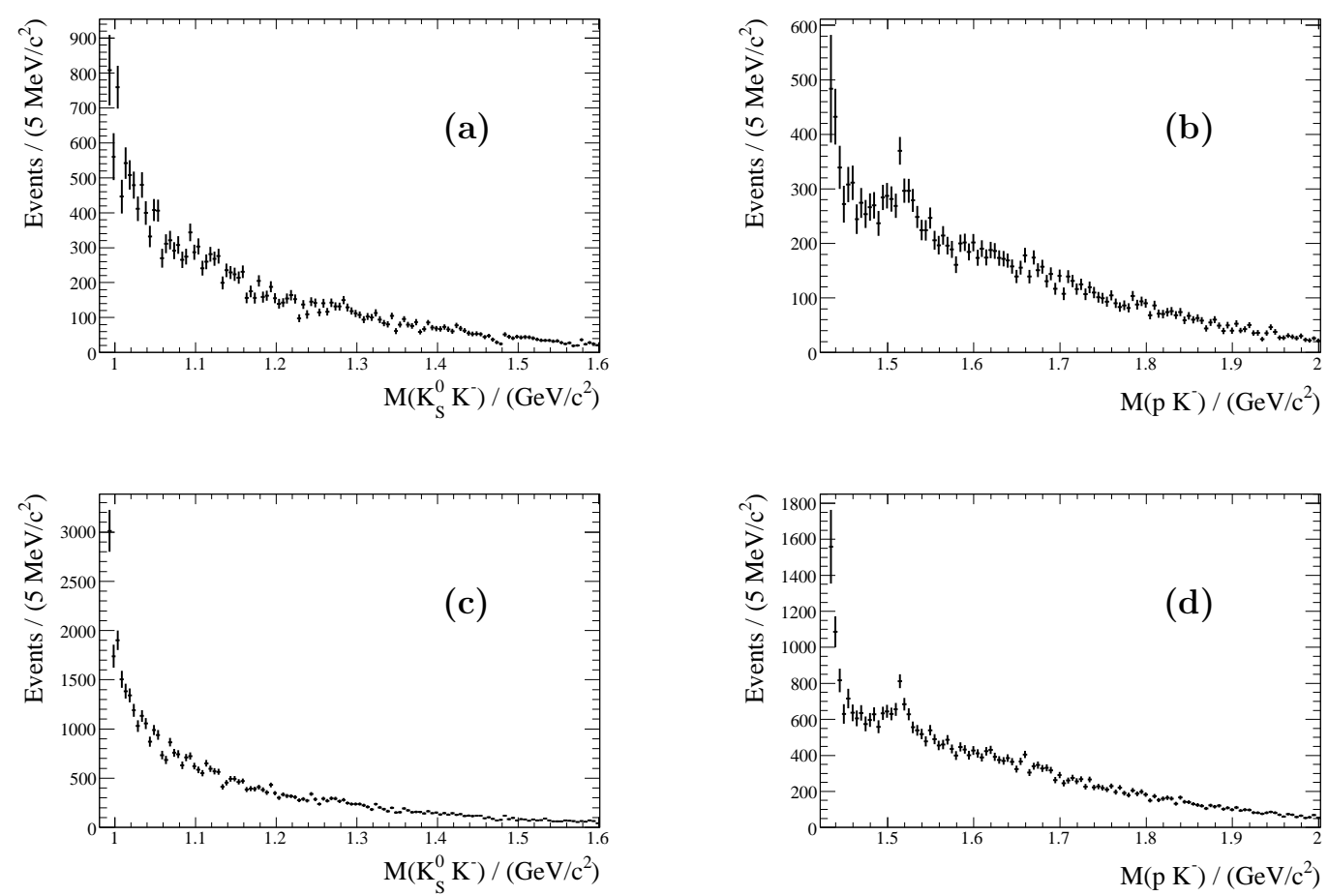

Figure 4.55: (a) The SBS $\mathrm{m}\left(K^{-} p\right)$, and (b) the SBS $\mathrm{m}\left(K^{-} K_{S}^{0}\right)$ distributions from figs. 4.50(b) and (d) weighted by (q0/q) for the incident electron region; (c) and (d) are the corresponding SBS distributions from figs. 4.38(b) and (d). 


\section{$e^{-} \operatorname{Be}\left(K_{S}^{0}, p\right)$ Candidates with an Associated $e^{-}$}

Negatively-charged tracks which are associated with an $e^{-} \mathrm{Be}\left(K_{S}^{0}, p\right)$ candidate vertex are chosen to be consistent with the electron mass hypothesis by requiring that they have $d E / d x(\mathrm{DCH})$ values in the range 500-850 counts (the electron plateau is at an average value of 675 counts). The scatterplot of $E / \mathrm{p}$ versus $\mathrm{p}$ for the selected tracks is shown in fig. 4.56(a), where $E$ is the energy of the associated EMC cluster and p is the magnitude of the lab. three-momentum of the track at the entrance to the EMC. A rather clear band for $E / \mathrm{p}$ near 1 and $\mathrm{p}>1 \mathrm{GeV} / c$ can be seen in the scatterplot, and tracks for which $0.9<E / \mathrm{p}<1.1$ with $\mathrm{p}>1 \mathrm{GeV} / c$ can thus be well-identified as electrons. The SBS $K_{S}^{0} p$ mass distribution for vertices with an identified electron and no baryon or $\bar{p}$ associated is shown in fig. 4.56 (b). There are $\sim 500$ surviving candidates in the plot, and yet again there is no sign of a $\Theta_{5}(1540)$ signal.
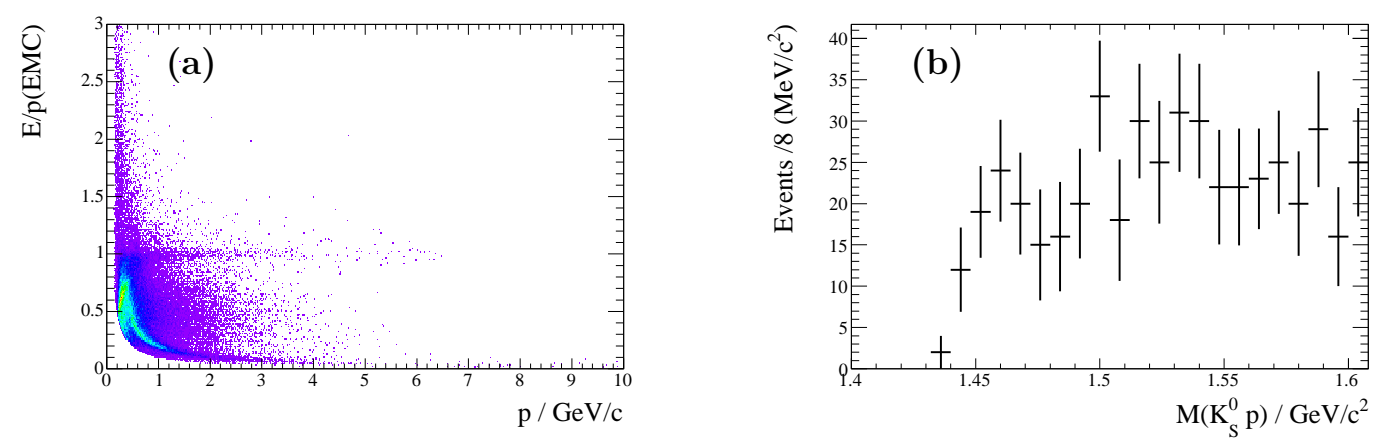

Figure 4.56: (a)The scatterplot of $(E / \mathrm{p})$ versus $\mathrm{p}$ for negatively-charged $\left(K_{S}^{0}, p\right)$ vertexassociated tracks for which $d E / d x(\mathrm{DCH})$ is consistent with that expected for an electron; (b) the SBS $K_{S}^{0} p$ mass distribution for $\left(K_{S}^{0}, p\right)$ vertices with an associated identified electron.

The fact that this number represents a very small fraction of the original $\sim 225000$ candidates is consistent with the interpretation that the electroproduction in the beampipe occurs predominantly at low $Q^{2}$ values i.e. that it corresponds to quasi-real photoproduction. 


\subsection{Comparison to Other Experiments}

\subsubsection{HERMES [49]}

Hermes $^{3}$ : Egyptian god of wisdom, fabled author of a number of mystical, philosophical and alchemistic writings.

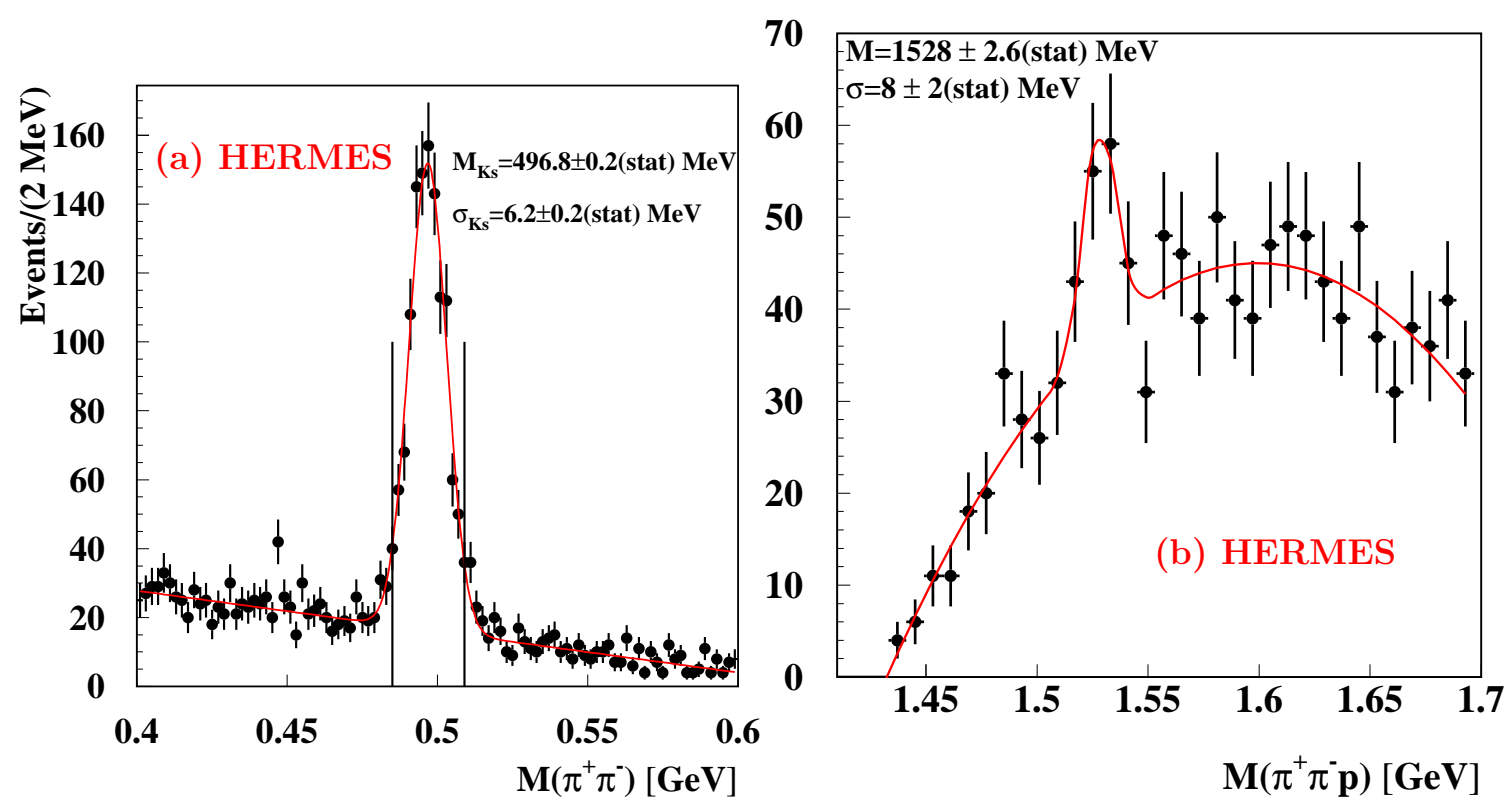

Figure 4.57: For the HERMES experiment, (a) the $\pi^{+} \pi^{-}$mass distribution in the $K_{S}^{0}$ region and, (b) the distribution in invariant mass of the $p \pi^{+} \pi^{-}$system for candidates with $\pi^{+} \pi^{-}$mass in the $K_{S}^{0}$ signal region indicated in fig. 4.57(a).

Of the experiments listed in Table 1.1, the HERMES experiment is the one most similar to the present electroproduction search. A comparison of the main features of the analyses is presented in Table 4.1. The BABAR target is more complex, but the mass resolution is significantly better, and in the $K_{S}^{0} p$ mass region less than $1.7 \mathrm{GeV} / c^{2}$ there are $\sim 227,174$ candidates for BABAR compared to 1171 in the HERMES distribution. Also it should be noted that the HERMES mass plot (fig. 4.57(b)) has not been corrected for background in the $K_{S}^{0}$ signal region.

In fig. 4.58(a) the BABAR electroproduction distribution of fig. 4.45(a) is compared to the HERMES distribution of fig. 4.57(b). For the BABAR data, the HERMES mass binning $\left(8 \mathrm{MeV} / c^{2}\right)$ has been chosen, and the distributions have been normalized in the $K_{S}^{0} p$ mass range 1.58-1.7 GeV/c $c^{2}$. The BABAR $K_{S}^{0}$ sideband distribution has not been subtracted since this has not been done in the HERMES analysis.

For the region above $1.52 \mathrm{GeV} / c^{2}$ the distributions seem consistent in shape, but at lower mass values the HERMES distribution deviates markedly from that for BABAR (and also from the corresponding ZEUS distribution; see fig. 4.58(b) and Section 4.8.2). There appears to be a significant loss of efficiency, which may result from the high momentum

\footnotetext{
${ }^{3}$ Webster's Dictionary
} 
Table 4.1: Comparison of the HERMES Analysis to the BABAR electroproduction analysis

\begin{tabular}{|c|c|c|}
\hline & $\begin{array}{r}\text { HERMES } \\
\text { PLB } 585(2004) 213\end{array}$ & $B A B A R$ Electroproduction \\
\hline $\begin{array}{l}\text { Incident } \\
\text { Beam }\end{array}$ & $e^{+} @ 27.6 \mathrm{GeV} / \mathrm{c}$ & $\begin{array}{l}e^{-} \sim 9 \mathrm{GeV} / \mathrm{c} \\
e^{+} \sim 3 \mathrm{GeV} / \mathrm{c}\end{array}$ \\
\hline Target & Polarized $\mathrm{D}_{2}$ Gas & $\mathrm{Be}\left[\right.$ some $\left.\mathrm{Ni}, \mathrm{Au}, \mathrm{H}_{2} \mathrm{O}, \mathrm{BR} 127\right]$ \\
\hline $\begin{array}{l}\text { Detect Scattered } \\
\text { Beam e }\end{array}$ & No & limited \\
\hline PID for $K_{S}^{0} \& \mathrm{p}$ & $\begin{array}{r}\text { RICH: p } 4-9 \mathrm{GeV} / \mathrm{c} \\
\pi 1-15 \mathrm{GeV} / \mathrm{c} \\
\end{array}$ & $d E / d x[\mathrm{SVT}$ and $\mathrm{DCH}]$ \\
\hline$\Lambda$ Mass Cut Needed & Yes & No \\
\hline $\operatorname{DOCA}(\pi \pi)$ & $<1.0 \mathrm{~cm}$ & $<0.3 \mathrm{~cm}+\mathrm{P}\left(\chi^{2}\right)>0.001$ \\
\hline $\operatorname{DOCA}\left(K_{S}^{0} \mathrm{p}\right)$ & $<0.6 \mathrm{~cm}$ & $<0.3 \mathrm{~cm}$ \\
\hline $\operatorname{SFL}\left(K_{S}^{0}\right)$ & $>7.0 \mathrm{~cm}$ & $>0.2 \mathrm{~cm}$ \\
\hline $\operatorname{Sigma}\left(K_{S}^{0}\right)$ & $\sim 6.2 \mathrm{MeV} / c^{2}$ & $\sim 2.8 \mathrm{MeV} / c^{2}$ \\
\hline Signal Region $\left(K_{S}^{0}\right)$ & $\pm 12.4 \mathrm{MeV} / \mathrm{c}^{2}$ & $\pm 10.0 \mathrm{MeV} / \mathrm{c}^{2}$ \\
\hline $\begin{array}{l}\text { Background in } \\
\text { Signal Region }\end{array}$ & $\sim 15 \%$ & $\sim 18 \%$ overall \\
\hline $\begin{array}{l}\operatorname{Sigma}\left(K_{S}^{0} \mathrm{p} \text { mass }\right) \\
\text { using PDG } K_{S}^{0} \text { mass }\end{array}$ & $\sim 4.3 \mathrm{MeV} / c^{2}$ & $\sim 2.0 \mathrm{MeV} / c^{2}$ \\
\hline $\begin{array}{l}\text { Number of Cands. } \\
\mathrm{m}\left(K_{S}^{0} \mathrm{p}\right)<1.7 \mathrm{GeV} / c^{2}\end{array}$ & 1171 & 227,174 \\
\hline $\begin{array}{l}\text { Other tracks } \\
\text { associated with } \\
\text { the }\left(K_{S}^{0} \mathrm{p}\right) \text { vertex? }\end{array}$ & $\mathrm{No}$ & $\begin{array}{l}\text { Yes; use } d E / d x, \text { DIRC } \\
\text { and EMC PID information }\end{array}$ \\
\hline $\begin{array}{l}K_{S}^{0} \text { Sidebands } \\
\text { Subtracted }\end{array}$ & No & Yes \\
\hline$\Theta_{5}^{+}$Signal? & $\begin{array}{r}\text { Yes; } \\
\text { claim } 4-6 \sigma \\
\text { depending on } \\
\text { background }\end{array}$ & No \\
\hline
\end{tabular}

requirements for RICH PID (Table 4.1: 4-9 GeV/c for protons, and 1-15 GeV/c for pions), the significant $K_{S}^{0}$ flight length requirement $(>7 \mathrm{~cm}$ compared to $>0.2 \mathrm{~cm}$ for $B A B A R)$, and the up-down separation of the detector. It then seems possible that 1-2 $\sigma$ fluctuations in the HERMES data in the 1.52-1.54 GeV/ $c^{2}$ region, and a sharp decrease in acceptance from $1.52 \mathrm{GeV} / c^{2}$ to threshold, might have conspired to generate a spurious signal in the $\Theta_{5}^{+}$mass region.

\subsubsection{ZEUS [50]}

The ZEUS experiment [50] makes use of $e^{+} p$ and $e^{-} p$ HERA Collider data at c.m. energies of 300 and $318 \mathrm{GeV}$ to investigate the combined $K_{S}^{0} p$ and $K_{S}^{0} \bar{p}$ mass distributions in the region below $1.7 \mathrm{GeV} / c^{2}$. The $K_{S}^{0} p$ and $K_{S}^{0} \bar{p}$ systems are centrally-produced and have $\mathrm{Q}^{2}$ greater than $20 \mathrm{GeV}^{2}$. The $K_{S}^{0}$ sample is large $(>800,000$ candidates $)$ and very clean $(\sim 6$ $\%$ background); however no information is provided on the flight-length criterion used nor 

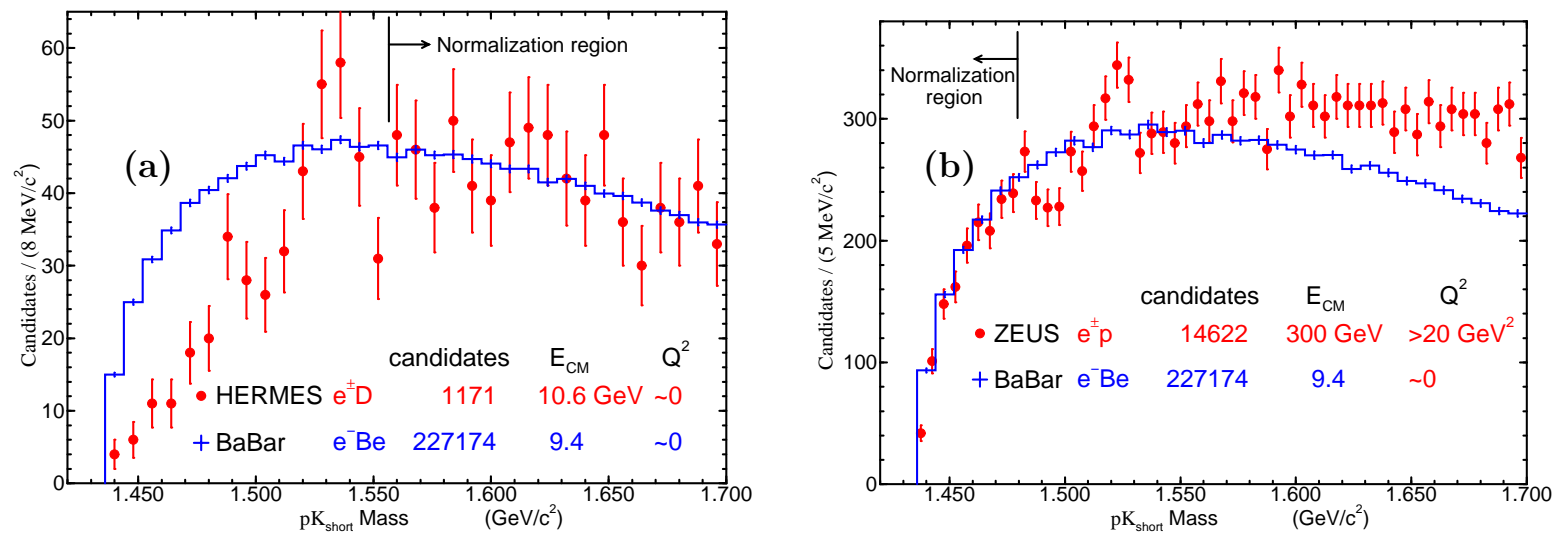

Figure 4.58: (a) The HERMES $K_{S}^{0} p$ mass distribution [49] compared to the BABAR distribution for electroproduction in Be normalized to the HERMES distribution above 1.58 $\mathrm{GeV} / c^{2}$. (b) The corresponding comparison for the ZEUS data [50]; the BABAR distribution has been normalized to the ZEUS data below $1.48 \mathrm{GeV} / c^{2}$.

on the range of lab. momentum detected. Proton and $\bar{p}$ identification are confined to lab. momenta less than $1.5 \mathrm{GeV} / c$, and rely on drift chamber $d E / d x$ (resolution $\sim 10 \%$ ). The selection procedure is very similar to that used in the BABAR analysis, but retains more pion and kaon background because of softer rejection criteria. For the ZEUS experiment, the final state electron is detected and identified, and required to have lab. energy greater than $8.5 \mathrm{GeV}$.

The ZEUS data for $\mathrm{Q}^{2}>20 \mathrm{GeV}^{2}$ are shown in fig. 4.58(b). Their fit to this distribution (fig.4 [50]) incorporates the $\Sigma(1480$ ) hyperon resonance (which PDG [9] considers to merit only one star i.e. it is of questionable pedigree!). The BABAR data in fig. 4.58(b) have been normalized to the ZEUS data with $K_{S}^{0} p$ mass below $1.48 \mathrm{GeV} / c^{2}$, and the distributions then agree very well in shape over the region of threshold rise. This reinforces the claim that the HERMES data in this region may suffer significant acceptance loss (fig. 4.58(a)), possibly as a result of their high-momentum-threshold PID requirements, especially for the proton. The BABAR data show no structure at the $\Sigma(1480)$ nor in the $\Theta_{5}^{+}$region, and fall below the ZEUS data above $\sim 1.55 \mathrm{GeV} / c^{2}$; the latter would be expected given the much higher ZEUS c.m. energies involved. The ZEUS fit gives a $\Theta_{5}^{+}$signal of 220 \pm 50 events; relative to the BABAR distribution a signal of only $\sim 130 \pm 40$ events is estimated, which suggests that the claimed observation of a significant $\Theta_{5}^{+}$signal is less than convincing.

\subsubsection{SAPHIR [42]}

In the SAPHIR experiment [42], the reaction

$$
\gamma p \rightarrow K^{+}(n) \bar{K}^{0}
$$


where the neutron was detected indirectly, was analyzed for incident photon energies in the range $\sim 0.9-2.6 \mathrm{GeV}$. The $\Theta_{5}^{+}$signal (fig. 2(a)[42]) is claimed to contain 55 events over 56 background in the four central mass bins $\left[\sim 58 \mathrm{MeV} / \mathrm{c}^{2}\right]$. The mass resolution is 23 $\mathrm{MeV} / \mathrm{c}^{2}$ (FWHM), and the undetected neutron is identified by means of a one-constraint kinematic fit; no corresponding missing mass distribution in the neutron region is shown, and the $\pi^{+} \pi^{-}$mass distribution for the $\bar{K}^{0}$ region is shown only for the $\Theta_{5}^{+}$signal region (fig. 3(a)[42]), for which the $K_{S}^{0} \mathrm{~S} / \mathrm{B}$ is only $\sim 1.3: 1$.

The SAPHIR final state particles are equivalent to the $\left(K_{S}^{0}, p, K^{-}\right)$electroproduction selection in $B A B A R$ if the $K_{S}^{0}$ is interpreted as a $K^{0}$, and so it is of interest to compare the distributions of $K^{-} p$ mass (fig. 4.50(d)) and $K_{S}^{0} p$ mass (fig. 4.51(b)) to those for $\bar{K}^{0} n$ mass (fig.2(b)[42]) and $K^{+} n$ mass (fig. 2(a)[42]) of the SAPHIR paper.

In refce. [42] fig.2(b) shows a $\Lambda(1520)$ signal of $\sim 600$ events, which is reduced to $\sim 150$ by requiring $\cos \theta_{\bar{K}^{0}}>0.5$ in the c.m. system. The $n K^{+}$distribution of fig.2(a) has had this criterion applied, but the authors indicate that the $\Theta_{5}^{+}$signal was reduced by about a factor of two as a consequence. This implies that the $\sim 450 \Lambda(1520)$ events with low lab. momentum $\bar{K}^{0}$ 's correspond to $\sim 50 \Theta_{5}^{+}$events. In the BABAR analysis, the $K^{-}$lab. momentum distribution is fairly soft, so that in this sense the $\sim 350$ event $\Lambda(1520)$ signal of fig. $4.50(\mathrm{~d})$ is equivalent to the low-momentum SAPHIR sample. This leads to a very crude estimate of $\sim 40-50 \Theta_{5}^{+}$events for fig. 4.51(b). For a $\Theta_{5}^{+}$width of $\sim 1 \mathrm{MeV}$ this would correspond to an excess of at most $\sim 30$ events for two adjacent mass bins, for which a one sigma statistical fluctuation in the $\Theta_{5}^{+}$region would be $\sim 14$ events i.e. the estimated signal would be equivalent to a background fluctuation of about $2 \sigma$ at most. It follows that on the basis of this admittedly crude reasoning the absence of a clear $\Theta_{5}^{+}$signal in fig. 4.51(b) cannot be considered to be in contradiction of the SAPHIR observation.

Of course since the new data from CLAS [55] on the SAPHIR reaction show no evidence of a $\Theta_{5}$ signal, the absence of a $\Theta_{5}$ signal in the $B A B A R$ quasi-real photoproduction data is in complete accord with the CLAS analysis. 


\section{Chapter 5}

\section{Conclusion}

The analysis presented in this thesis has described a search for the production of the $\Theta_{5}(1540)^{+}$strange pentaquark candidate using data collected with the BABAR detector at the asymmetric-energy PEP II $e^{+} e^{-}$Collider at SLAC. The $p K_{S}^{0}$ system, with $K_{S}^{0} \rightarrow$ $\pi^{+} \pi^{-}$, has been investigated for evidence of $\Theta_{5}$ production in $e^{+} e^{-}$collisions at, and 0.04 $\mathrm{GeV}$ below, c.m. energy $10.58 \mathrm{GeV}$, and also in the interactions of beam halo electrons and positrons and of secondary hadrons from primary $e^{+} e^{-}$collisions in the material of the $B A B A R$ detector. In the former search, the data sample used corresponded to an integrated luminosity of $\sim 123.5 \mathrm{fb}^{-1}$ from Run Periods 1-3, while in the latter the full data sample from Run Periods 1-4, with associated integrated $e^{+} e^{-}$luminosity of $\sim 230$ $\mathrm{fb}^{-1}$, was employed.

The $p K_{S}^{0}$ invariant mass distribution obtained in the $e^{+} e^{-}$analysis showed a large ( $\sim 100,000$ events) signal corresponding to the production of the $\Lambda_{c}(2285)^{+}$charmed baryon, and this was used to verify that the dependence of mass resolution upon c.m. momentum, $p^{*}$, was well- reproduced in Monte Carlo simulation. The same simulation in the mass region of the $\Theta_{5}(1540)^{+}$yielded mass resolution values in the range 1.7-2.2 $\mathrm{MeV} / \mathrm{c}^{2}$, and the excellent agreement obtained for the $\Lambda_{c}(2285)^{+}$data demonstrates that these resolution estimates are reliable. No $\Theta_{5}(1540)^{+}$signal was observed, neither when the $p K_{S}^{0}$ mass distribution was taken as a whole, nor when it was examined as a function of $p^{*}$. This remained true for sub-samples of the data for which each event was required to contain an identified $K^{-}$(in order to bias the $K_{S}^{0}$ sample toward $K^{0}$ rather than $\bar{K}^{0}$ ), and/or an anti-proton (in order to bias the sample toward conserved baryon number).

For a fixed $\Theta_{5}(1540)^{+}$mass value of $1.54 \mathrm{GeV} / c^{2}$, and fixed total width values of 1 $\mathrm{MeV}$ and $8 \mathrm{MeV}$, resolution-smeared P-wave Breit-Wigner lineshapes were used to obtain $95 \%$ c.l. cross section upper limits for each $0.5 \mathrm{GeV} / c p^{*}$ interval in the range $0-5 \mathrm{GeV} / c$. Limits for the integrated cross section values of $\sim 180 \mathrm{fb}$ and $\sim 360 \mathrm{fb}$ were extracted for width values $1 \mathrm{MeV}$ and $8 \mathrm{MeV}$ respectively. The values obtained for a mass choice of $1.53 \mathrm{GeV} / c^{2}$ were virtually identical to these. These upper limit values are significantly below the cross section values which would be expected for a particle of mass $1.54 \mathrm{GeV} / c^{2}$ on the basis of the observed production rates for "ordinary" hadrons (see Chapter 3, 
fig. 3.23). This suggests that if the $\Theta_{5}(1540)^{+}$pentaquark state does in fact exist, its production is highly suppressed in $e^{+} e^{-}$interactions at $\sim 10.58 \mathrm{GeV}$ with respect to that of well-established hadrons.

The second analysis, described in Chapter 4, extended the search for the $\Theta_{5}(1540)^{+}$ to the interactions of secondary hadrons, background tracks of every type, and beam halo electrons and positrons in the material of the inner BABAR detector.

It has been demonstrated that the candidate $\left(K_{S}^{0}, p\right)$ vertices reproduce the detector geometry very well, however the inclusive $K_{S}^{0} p$ mass distribution shows no signal.

Sub-samples of the candidates with at least one associated charged track, and also those remaining after rejecting $\left(K_{S}^{0}, p\right)$ vertices with at least one associated baryon have been examined. The requirement of an associated $\pi^{ \pm}$or $K^{ \pm}$has resulted in the observation of signals for $K^{*}(892)^{+}, \bar{K}^{*}(892)^{-}, \Lambda(1115), \Lambda(1520)$ and indications of coupling to $a_{0}(980)^{-}$ and $\Lambda(1405)$. In each instance, the corresponding $K_{S}^{0} p$ distribution shows no evidence of a $\Theta_{5}(1540)^{+}$signal.

The study has been restricted to the regions which can be interpreted as corresponding to electroproduction in Be (mainly) and Ta and all of the analyses have been repeated, including in addition a small sample of vertices with an associated electron. Again no $\Theta_{5}(1540)^{+}$signal has been observed.

Since there is no quantitative information on the flux of off-beam electrons and positrons, it is not possible to estimate upper limits for production cross section as was done in refce. [59] and in Chapter 3 of this thesis.

The BABAR electroproduction results have been compared to those of the HERMES[49] and ZEUS[50] experiments. These comparisons seem to indicate a significant loss of acceptance for the HERMES experiment in the $K_{S}^{0} p$ mass region below $\sim 1.52 \mathrm{GeV} / c^{2}$. There is no evidence for the $\Sigma(1480)$ and $\Theta_{5}^{+}$signals of the ZEUS analysis, and this creates serious reservations about the significance of the $\Theta_{5}^{+}$observations claimed by HERMES and ZEUS.

Finally, the BABAR results on the electroproduction of the $K_{S}^{0} p K^{-}$system have been compared to the SAPHIR[42] results on the photoproduction of the $K^{+} n \bar{K}^{0}$ final state. A crude attempt at normalizing the production of $\Lambda(1520)$ observed in both analyses leads to the conclusion that a $\Theta_{5}^{+}$signal as observed by SAPHIR would not be significant in the corresponding BABAR $K_{S}^{0} p$ mass distribution. The BABAR results are of course in complete accord with those presented recently by the CLAS Collaboration [55].

In summary, the end result of the many high statistics searches described in this thesis is that none of them has yielded any evidence for the production of the $\Theta_{5}(1540)^{+}$. Furthermore, a comparison of of the BABAR results on electroproduction in Be to those from the HERMES $\left(e^{+} \mathrm{D}\right)$ and ZEUS $\left(e^{ \pm} \mathrm{p}\right)$ experiments leads to the conclusion that prior claims for the observation of $\Theta_{5}(1540)^{+}$electroproduction are less than convincing.

In light of the present results on the candidate pentaquark states it would seem clear that the only way that this whole issue can be resolved is for the experiments which at 
present claim a signal to be re-run with higher statistics. However, many of the analyses have been carried out in experiments which are now decommissioned. Nevertheless, for those which can be repeated, there is a clear need for new high statistics data to be collected with well-calibrated, large-acceptance detectors. Proof of principle has been amply provided by the new results from CLAS [55], which so convincingly refute the earlier claim of $\Theta_{5}^{+}$observation from SAPHIR [42]. 


\section{Bibliography}

[1] M.Gell-Mann, Phys. Lett. 8, 214 (1964)

[2] G.Zweig, CERN preprints TH-401 and TH-412 (1964)

[3] M.Gell-Mann, Calif. Inst. of Technology Lab. Report, CTSL-20 (1961)

[4] Y.Ne'eman , Nucl. Phys. 26, 222 (1961)

[5] M.Gell-Mann, Phys. Rev. 125, 1067 (1962)

[6] M.Gell-Mann, p.805, Proceedings of the International Conference on High-Energy Physics, CERN (1962)

[7] V.E.Barnes et al., Phys. Rev. Lett. 12, 204 (1964)

[8] Y.Eisenberg, Phys. Rev. 96, 541 (1954)

[9] S.Eidelman et al.(PDG2004), Phys. Lett. B592, 1 (2004)

[10] S.Godfrey and N.Isgur, Phys. Rev. D32, 189 (1985)

[11] J.Weinstein and N.Isgur, Phys. Rev. Lett. 48, 659 (1982); Phys. Rev. D27, 588 (1983)

[12] R.L.Jaffe, Phys. Rev. D15, 267 (1977); Phys. Rev. D15, 281 (1977); Phys. Rev. D17, 1444 (1978); R.L.Jaffe and K.Johnson, Phys. Lett. B60, 201 (1976)

[13] C.Morningstar and M.Peardon, Phys. Rev. D60, 034509 (1999)

[14] W.Dunwoodie, SLAC-PUB-7163 (1997) and references therein

[15] N.Isgur and J.Paton, Phys. Rev. D31, 2910 (1985)

[16] P.Laycock et al., Phys.Lett. B401, 308 (1997); C.Bernard et al., Phys. Rev. D56, 7039 (1997)

[17] T.Barnes et al, Nucl. Phys. B224, 241 (1983)

[18] D.R.Thompson et al., Phys. Rev. Lett. 79, 1630 (1997); S.U.Chung et al., Phys. Rev. D60, 092001 (1999)

[19] G.S.Adams et al., Phys. Rev. Lett. 81, 5760 (1998); E.I.Ivanov et al., Phys. Rev. Lett. 86, 3977 (2001) 
[20] R.Koniuk and N.Isgur, Phys. Rev. D21, 1868 (1980); this contains many references to the Isgur-Karl non-relativistic potential model, and to the original work of Greenberg, Dalitz and others.

[21] A.J.G.Hey and R.L.Kelly, Phys. Reports 96, 71 (1983)

[22] M.Aguilar-Benitez et al. (PDG1986), Phys. Lett. B170, 1 (1986)

[23] G.P.Yost et al. (PDG1988), Phys. Lett. B204, 1 (1988)

[24] T.H.R.Skyrme, Nucl. Phys. 31, 556 (1962)

[25] D. Diakonov, V. Petrov and M.V. Polyakov, Z. Phys. A 359, 305 (1997)

[26] J.Napolitano et al., hep-ex/0412031 (2004) and references therein

[27] A.Berthon et al., Nucl. Phys. B63, 54 (1973)

[28] G.Trilling, Phys. Lett. B592, 916 (2004) (PDG 2004)

[29] T.Nakano (LEPS), PANIC 2002 Proceedings, NP A721, 112c (2003)

[30] C.Alt et al. (NA49), Phys. Rev. Lett. 92, 042003 (2004)

[31] D.Diakonov and V.Petrov, Phys. Rev. D69, 094011 (2004)

[32] M.Karliner and H.Lipkin, hep-ph/0307243 (2003)

[33] R.Jaffe and F.Wilczek, Phys. Rev. Lett. 91, 232003 (2003)

[34] K.Holland and K.Juge, hep-lat/0504007 (2005)

[35] Y.Oh et al., Phys. Rev. D69, 014009 (2004)

[36] Y.Oh et al., Nucl. Phys. A745, 129 (2004)

[37] T.Nakano et al. (LEPS), Phys. Rev. Lett. 91, 012002 (2003)

[38] T. Nakano (LEPS), Presented at QNP2004, Indiana U. 2004 (http://www.qnp2004.org)

[39] A.R.Dzierba et al., presented at the First Meeting of the APS Topical Group on Hadronic Physics (2004); hep-ex/0412077 (2004)

[40] S. Stepanyan et al (CLAS (d)), Phys. Rev. Lett. 91, 252001 (2003)

[41] V. Kubarovsky et al (CLAS (p)), Phys. Rev. Lett. 92, 032001-1 (2004)

[42] J. Barth et al (SAPHIR), Phys. Lett. B572, 127 (2003)

[43] M. Abdel-Bary et al (COSY), Phys. Lett. B595, 127 (2004)

[44] P.Aslanyan et al. (JINR), hep-ex/0403044 (2004) 
[45] A. Aleev et al. (SVD), hep-ex/0401024 (2004)

[46] V. Barmin et al. (DIANA) Phys. Atom. Nucl. 66, 1715 (2003)

[47] A. Asratyan, et al. (ITEP), Phys. Atom. Nucl. 67, 682 (2004)

[48] L.Camilleri (NOMAD), presented at Neutrino 2004, Paris (2004) (http://neutrino2004.in2p3.fr/program.htm)

[49] A. Airapetian et al. (HERMES), Phys. Lett. B585, 213 (2004)

[50] S. Chekanov et al. (ZEUS), Phys. Lett. B591, 7 (2004)

[51] S.Nussinov, hep-ph/0307357 (2003); Phys. Rev. D69, 116001 (2004)

[52] R.A.Arndt et al., Phys. Rev. C68, 042201 (2003)

[53] J.Haidenbauer and G.Krein, Phys. Rev. C68, 052201 (2003)

[54] R.N.Cahn and G.H.Trilling, Phys. Rev. D69, 011501(R) (2004)

[55] R.DeVita (CLAS(p,2)), presented at the APS Meeting, Tampa, Florida (2005); (link from: http://www.phy.ohiou.edu/ hicks/thplus.html)

[56] H.G.Fischer, S.Wenig. Eur. Phys.J. C37, 133 (2004)

[57] A.Aktas et al et al. (H1), Phys. Lett. B588, 17 (2004)

[58] S.Schael et al. (ALEPH), Phys. Lett. B599, 1 (2004); CERN-PH-EP-2004-038

[59] B.Aubert et al. (BABAR), hep-ex/0408064 (2004); hep-ex/0502004 (submitted to Phys. Rev. Lett.) (2005)

[60] R.Mizuk et al. (BELLE), hep-ex/0411005 (2004)

[61] J.Bai et al. (BES), Phys. Rev. D70, 012004 (2004); hep-ex/0402012 (2004)

[62] M.-J.Wang et al. (CDF), presented at QNP2004: (http://www.qnp2004.org;)

I.Gorelove et al. (CDF), hep-ex/0408025 (2004);

D.Litvintsev et al. (CDF), hep-ex/0410024 (2004)

[63] G.Brona and B.Badelek (COMPASS): (http://wwwcompass.cern.ch/compass/notes/2004-5)

[64] C.Lin (DELPHI), presented at ICHEP2004: http://ichep04.ihep.ac.cn

[65] D.Christian et al. (E690), presented at QNP2004 (2004): (http://www.qnp2004.org) 
[66] K.Stenson (FOCUS), hep-ex/0412021 (2004)

[67] I.Abt et al. (HERA-B), Phys. Rev. Lett. 93, 212003 (2004) and hep-ex/0408048 (2004);

K.Knoepfle et al. (HERA-B), J. Phys. G30, S1363 (2004) and hep-ex/0403020 (2004)

[68] M.Longo et al. (HyperCP), hep-ex/0410027 (2004)

[69] S.Armstrong et al. (L3), hep-ex/0410080 (2004)

[70] C.Pinkenburg et al. (PHENIX), J. Phys. G30, S1201 (2004) and nucl-ex/0404001 (2004)

[71] J.Engelfried (SELEX), presented at Quark Confinement 2004 (2004):

(http://www.eurocongress.it/Quark)

[72] Y.Antipov et al. (SPHINX), Eur. Phys. J. A21, 455 (2004) and hep-ex/0407026 (2004)

[73] S.Salur et al. (STAR), hep-ex/0403009 (2004) and nucl-ex/0410039 (2004)

[74] M.Adamovich et al. (WA89), hep-ex/0405042 (2004)

[75] ZEUS Collaboration, presented at ICHEP2004 (2004):

(http://ichep04.ihep.ac.cn)

[76] S.Chekanov et al. (ZEUS), Eur. Phys. J. C38, 29 (2004) and hep-ex/0409033 (2004)

[77] P.Robbe, Ph.D Thesis, LAPP Annecy, LAPP-T-2002-01 (2002)

[78] P.Oddone, in Proceedings of the UCLA Workshop: Linear Collider B $\bar{B}$ factory conceptual Design, edited by D.Stork, World Scientific (1987), p. 243

[79] B. Aubert et al. (BABAR ), Nucl. Instrum. Meth. A479, 1 (2002)

[80] C. Alt et al, (NA49)

(http://na49info.cern.ch/cgi-bin/wwwd-util/NA49/NOTE?294)

[81] Short write-up, describing status and new physics as of PYTHIA 6.154: T Sjöstrand, P. Edén, C. Friberg, L. Lönnblad, G. Miu, S. Mrenna, E. Norrbin Computer Phys. Commun. 135 (2001) 238 (LU TP 00-30, hep-ph/0010017)

[82] http://www.slac.stanford.edu/BFROOT/www/Physics/TrackEfficTaskForce/TrackingTaskForce2004.

[83] Brandon Hartfiel, Ph.D. Thesis, UCLA; to be submitted (2005)

[84] F. James and M. Roos, "'Minuit' A System For Function Minimization And Analysis Of The Parameter Errors And Correlations," Comput. Phys. Commun. 10, 343 (1975)

[85] M. Karliner and H. Lipkin, hep-ph/0402260 (2004) 